Trends and Habitat Associations of Waterbirds Using the South Bay Salt Pond Restoration Project, San Francisco Bay, California

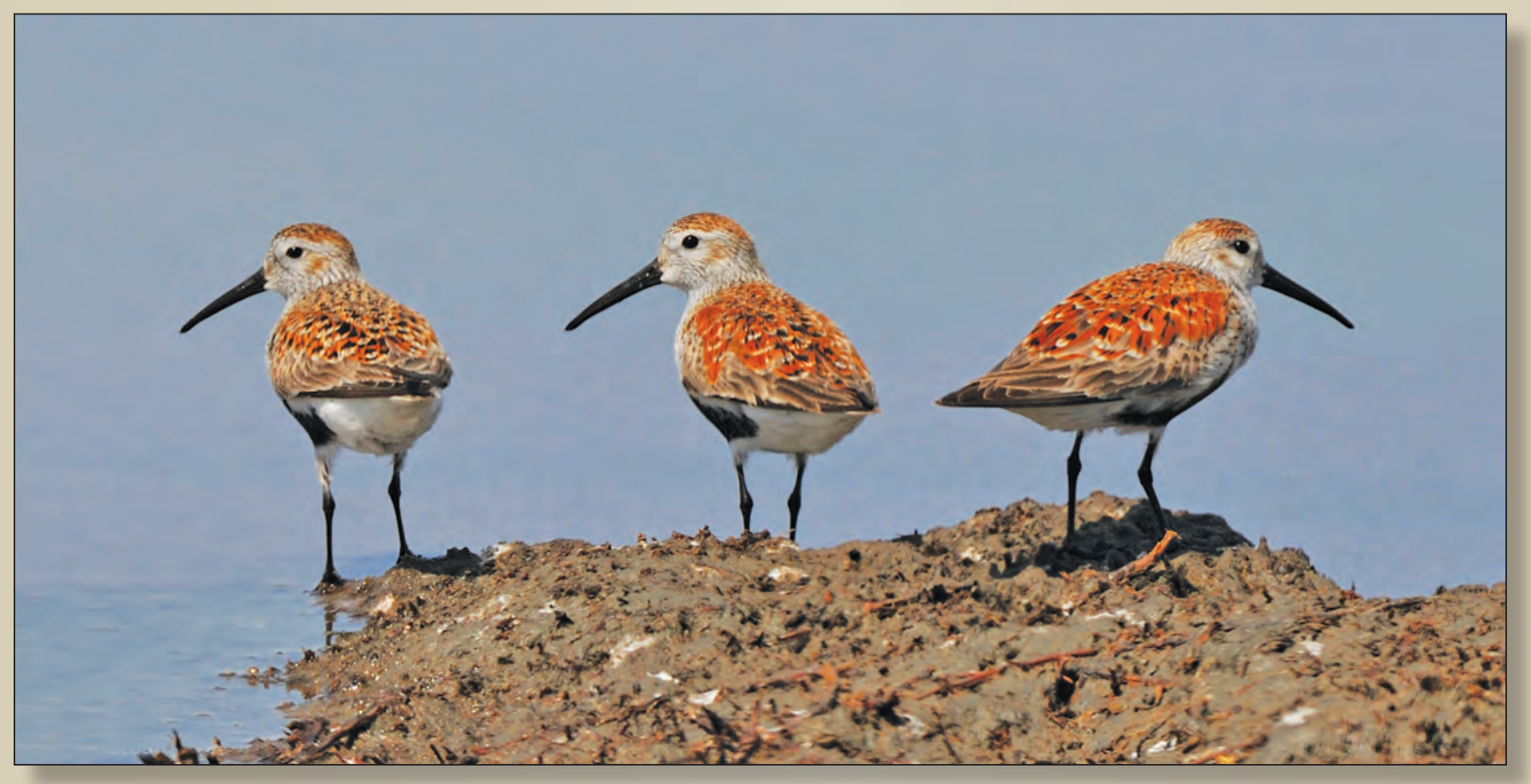

Open-File Report 2018-1040 
Cover: Dunlin (Calidris alpina) in a South San Francisco Bay managed pond. Photograph by Kathleen Henderson and used with permission. 


\section{Trends and Habitat Associations of Waterbirds Using the South Bay Salt Pond Restoration Project, San Francisco Bay, California}

By Susan E.W. De La Cruz, Lacy M. Smith, Stacy M. Moskal, Cheryl Strong, John Krause, Yiwei Wang, and John Y. Takekawa

Open-File Report 2018-1040

U.S. Department of the Interior

U.S. Geological Survey 


\section{U.S. Department of the Interior \\ RYAN K. ZINKE, Secretary}

\section{U.S. Geological Survey \\ William H. Werkheiser, Deputy Director \\ exercising the authority of the Director}

U.S. Geological Survey, Reston, Virginia: 2018

For more information on the USGS-the Federal source for science about the Earth, its natural and living resources, natural hazards, and the environment-visit https://www.usgs.gov/ or call 1-888-ASK-USGS (1-888-275-8747).

For an overview of USGS information products, including maps, imagery, and publications, visit https:/store.usgs.gov.

Any use of trade, firm, or product names is for descriptive purposes only and does not imply endorsement by the U.S. Government.

The findings and conclusions in this article are those of the author(s) and do not necessarily represent the views of the U.S. Fish and Wildlife Service.

Although this information product, for the most part, is in the public domain, it also may contain copyrighted materials as noted in the text. Permission to reproduce copyrighted items must be secured from the copyright owner.

Suggested citation:

De La Cruz, S.E.W., Smith, L.M., Moskal, S.M., Strong, C., Krause, J., Wang, Y., and Takekawa, J.Y., 2018,

Trends and habitat associations of waterbirds using the South Bay Salt Pond Restoration Project, San Francisco Bay, California: U.S. Geological Survey Open-File Report 2018-1040, 136 p., https://doi.org/10.3133/ofr20181040.

ISSN 2331-1258 (online) 


\section{Contents}

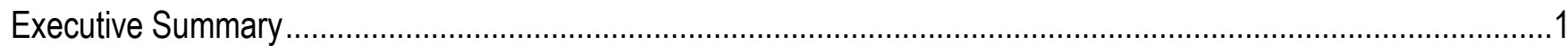

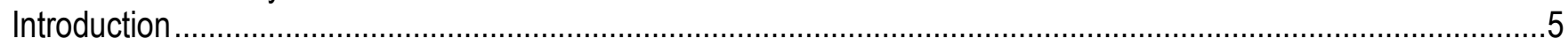

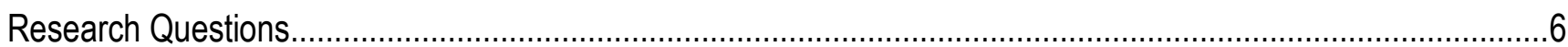

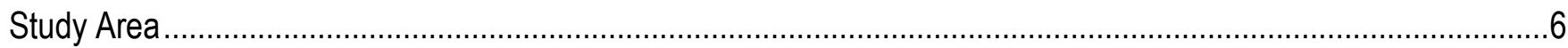

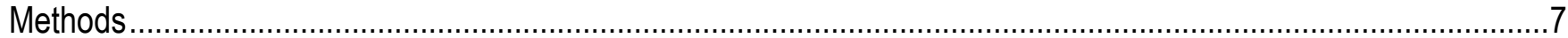

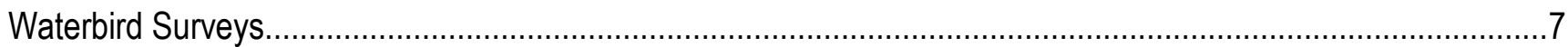

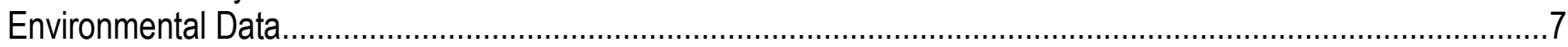

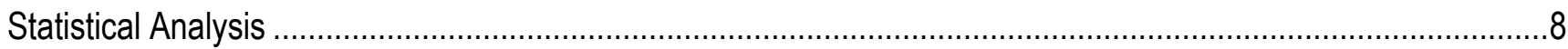

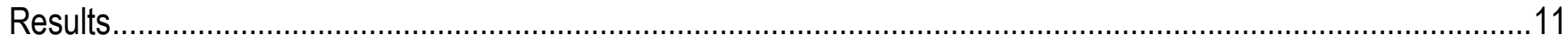

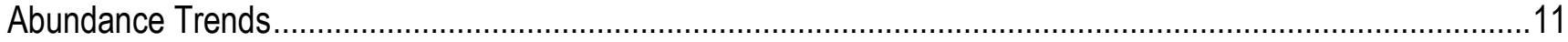

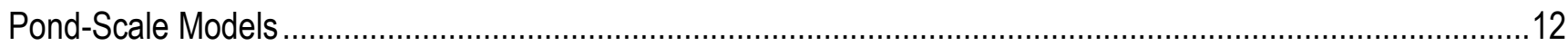

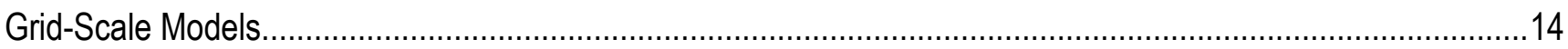

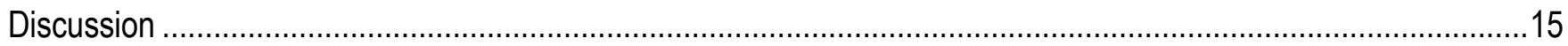

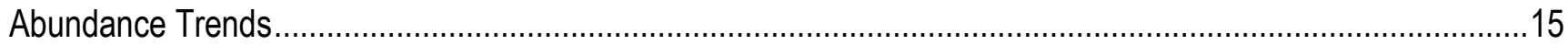

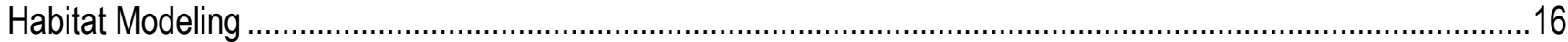

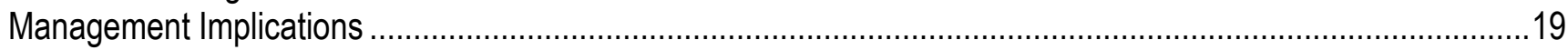

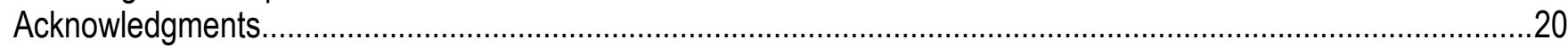

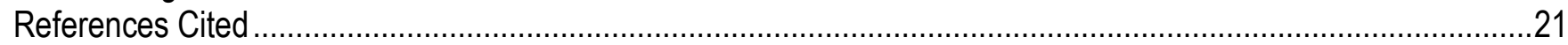

\section{Figures}

Figure 1. Former and current salt ponds grouped in several complexes in South San Francisco Bay, California . 25

Figure 2. Dabbling duck population trends during fall, winter, and spring in South Bay Salt Pond Restoration Project ponds and active salt production ponds, South San Francisco Bay, California .......................................26

Figure 3. Diving duck population trend during fall, winter, and spring in South Bay Salt Pond Restoration Project ponds and active salt production ponds, South San Francisco Bay, California ...............................................2 27

Figure 4. Small shorebird population trend during fall, winter, and spring in South Bay Salt Pond Restoration Project ponds and active salt production ponds, South San Francisco Bay, California ..................................... 28

Figure 5. Medium shorebird population trend during fall, winter, and spring in South Bay Salt Pond Restoration Project ponds and active salt production ponds, South San Francisco Bay, California.

Figure 6. Wader population trend during fall, winter, and spring in South Bay Salt Pond Restoration Project ponds and active salt production ponds, South San Francisco Bay, California

Figure 7. Piscivore population trend during fall, winter, and spring in South Bay Salt Pond Restoration Project ponds and active salt production ponds, South San Francisco Bay, California

Figure 8. Tern population trend during fall, winter, and spring in South Bay Salt Pond Restoration Project ponds and active salt production ponds, South San Francisco Bay, California.

Figure 9. Gull population trend during fall, winter, and spring in South Bay Salt Pond Restoration Project ponds and active salt production ponds, South San Francisco Bay, California.

Figure 10. Eared grebe population trend during fall, winter, and spring in South Bay Salt Pond Restoration Project ponds and active salt production ponds, South San Francisco Bay, California. 34 Figure 11. Northern shoveler population trend during fall, winter, and spring in South Bay Salt Pond Restoration Project ponds and active salt production ponds, South San Francisco Bay, California 
Figure 12. Ruddy duck population trend during fall, winter, and springin South Bay Salt Pond Restoration Project ponds and active salt production ponds, South San Francisco Bay, California 36

Figure 13. American avocet population trend during fall, winter, and spring in South Bay Salt Pond Restoration Project ponds and active salt production ponds, South San Francisco Bay, California

Figure 14. Model-averaged coefficients of pond water depth for foraging and roosting waterbirds at the pond scale in restoration ponds, South San Francisco Bay, California.

Figure 15. Diving duck, medium and small shorebird, gull, piscivore and wader abundance response to water depth from generalized additive mixed models at the pond scale in restoration ponds, South San Francisco Bay, California

Figure 16. Model-averaged coefficients of pond salinity for foraging and roosting waterbirds at the pond scale in restoration ponds, South San Francisco Bay, California. Coefficients are considered significant only if the 95-percent $\mathrm{Cl}$ does not overlap zero.

Figure 17. Dabbling duck, diving duck, gull, piscivore, tern, and wader abundance response to salinity from generalized additive mixed models at the pond scale in restoration ponds, South San Francisco Bay, California .. 42 Figure 18. Model-averaged coefficients of pond area for foraging and roosting waterbirds at the pond scale in restoration ponds, South San Francisco Bay, California. 44

Figure 19. Diving duck, gull, piscivore, tern, and wader abundance response to pond area from generalized additive mixed models at the pond scale in restoration ponds, South San Francisco Bay, California .

Figure 20. Model-averaged coefficients of pond topography for foraging and roosting waterbirds at the pond scale in restoration ponds, South San Francisco Bay, California.

Figure 21. Roosting dabbling duck and roosting medium shorebird abundance response to pond topography from generalized additive mixed models at the pond scale in restoration ponds, South San Francisco Bay, California

Figure 22. Model-averaged coefficients of islands for foraging and roosting waterbirds at the pond scale in restoration ponds, South San Francisco Bay, California. Coefficients are considered significant only if the 95-percent $\mathrm{Cl}$ does not overlap zero.

Figure 23. Model-averaged coefficients of distance to San Francisco Bay for foraging and roosting waterbirds at the pond scale in restoration ponds, South San Francisco Bay, California.

Figure 24. Diving duck and gull abundance response to pond distance to San Francisco Bay from generalized additive mixed models at the pond scale in restoration ponds, South San Francisco Bay, California .....

Figure 25. Gull abundance response to distance to landfill from generalized additive mixed models at the pond scale in restoration ponds, South San Francisco Bay, California.

Figure 26. Model-averaged coefficients of percent of pond levees open to hunting for foraging and roosting waterbirds at the pond scale in restoration ponds, South San Francisco Bay, California ....

Figure 27. Dabbling duck, gull, piscivore, tern, and wader abundance response to percentage of levees open to hunting from generalized additive mixed models at the pond scale in restoration ponds, South San Francisco Bay, California

Figure 28. Model-averaged coefficients of percentage of pond levees open to the public for foraging and roosting waterbirds at the pond scale in restoration ponds, South San Francisco Bay, California.......

Figure 29. Small shorebird and gull abundance response to percentage of levees open to the public from generalized additive mixed models at the pond scale in restoration ponds, South San Francisco Bay, California .. 57 Figure 30. Model-averaged coefficients of distance to the urban areas for foraging and roosting waterbirds at the pond scale in restoration ponds, South San Francisco Bay, California.

Figure 31. Model-averaged coefficients of pond management compared to not breached for foraging and roosting waterbirds at the pond scale in restoration ponds, South San Francisco Bay, California.

Figure 32. American avocet abundance response to depth and topography from generalized additive mixed models at the pond scale in restoration ponds, South San Francisco Bay, California 
Figure 33. Northern shoveler abundance response to salinity, percentage of the levee open to hunting, and pond topography from generalized additive mixed models at the pond scale in restoration ponds, South San Francisco Bay, California

Figure 34. Ruddy duck abundance response to water depth, salinity, pond area, and distance to San Francisco Bay from generalized additive mixed models at the pond scale in restoration ponds, South San Francisco Bay, California

Figure 35. Eared grebe abundance response to depth, salinity, and pond area from generalized additive mixed models at the pond scale in restoration ponds, South San Francisco Bay, California

Figure 36. Model-averaged coefficients for foraging and roosting eared grebes in salt production ponds at the pond scale in restoration ponds, South San Francisco Bay, California.

Figure 37. Model-averaged coefficients of grid water depth for foraging and roosting waterbirds at the grid scale in restoration ponds, South San Francisco Bay, California.

Figure 38. Dabbling duck, diving duck, medium and small shorebird, gull, piscivore, tern, and wader abundance response to water depth from generalized additive models at the grid scale in restoration ponds, South San Francisco Bay, California

Figure 39. Model-averaged coefficients of islands for foraging and roosting waterbirds at the grid scale in restoration ponds, South San Francisco Bay, California

Figure 40. Model-averaged coefficients of grid distance to levee for foraging and roosting waterbirds at the grid scale in restoration ponds, South San Francisco Bay, California.

Figure 41. Medium and small shorebird, gull, piscivore, tern, and wader abundance response to grid distance to levees from generalized additive models at the grid scale in restoration ponds, South San Francisco Bay, California

Figure 42. Model-averaged coefficients of grid distance to creek for foraging and roosting waterbirds at the grid scale in restoration ponds, South San Francisco Bay, California.

Figure 43. Medium shorebird, small shorebird and piscivore abundance response to grid distance to adjacent creek from generalized additive models at the grid scale in restoration ponds, South San Francisco Bay, California

Figure 44. Model-averaged coefficients of grid topography for foraging and roosting waterbirds at the grid scale in restoration ponds, South San Francisco Bay, California

Figure 45. Dabbling duck, diving duck, medium and small shorebird, gull, wader abundance response to pond topography from generalized additive models at the grid scale in restoration ponds, South San Francisco Bay, California

Figure 46. American avocet abundance response to depth, distance to nearest creek, distance to levees, and pond topography from generalized additive mixed models at the grid scale in restoration ponds, South San Francisco Bay, California...

Figure 47. Northern shoveler abundance response to depth, distance to levees, and pond topography from generalized additive mixed models at the grid scale in restoration ponds, South San Francisco Bay, California .... 78 Figure 48. Ruddy duck abundance response to depth and distance to levees from generalized additive mixed models at the grid scale in restoration ponds, South San Francisco Bay, California ...

Figure 49. Eared grebe abundance response to depth and distance to levees from generalized additive mixed models at the grid scale in restoration ponds, South San Francisco Bay, California 


\section{Tables}

Table 1. Attributes of the South Bay Salt Pond Restoration Project ponds used in waterbird habitat association models, South San Francisco Bay, California...

Table 2. Common name, scientific name, and associated foraging guild of waterbird species observed on salt ponds, South San Francisco Bay, California, October-April 2002-13.

Table 3. The ranking of candidate models at the pond scale for foraging dabbling ducks in the former salt production ponds of South San Francisco Bay, California.....

Table 4. The ranking of candidate models at the pond scale for roosting dabbling ducks in the former salt production ponds of South San Francisco Bay, California

Table 5. The ranking of candidate models at the pond scale for foraging and roosting diving ducks in the former salt production ponds of South San Francisco Bay, California

Table 6. The ranking of candidate models at the pond scale for foraging small shorebirds in the former salt production ponds of South San Francisco Bay, California..

Table 7. The ranking of candidate models at the pond scale for roosting small shorebirds in the former salt production ponds of South San Francisco Bay, California.

Table 8. The ranking of candidate models at the pond scale for foraging medium shorebirds in the former salt production ponds of South San Francisco Bay, California...

Table 9. The ranking of candidate models at the pond scale for roosting medium shorebirds in the former salt production ponds of South San Francisco Bay, California

Table 10. The ranking of candidate models at the pond scale for foraging wadersa in the former salt production ponds of South San Francisco Bay, California.

Table 11. The ranking of candidate models at the pond scale for roosting waders in the former salt production ponds of South San Francisco Bay, California.

Table 12. The ranking of candidate models at the pond scale for foraging and roosting piscivores in the former salt production ponds of South San Francisco Bay, California

Table 13. The ranking of candidate models at the pond scale for foraging terns in the former salt production ponds of South San Francisco Bay, California.

Table 14. The ranking of candidate models at the pond scale for roosting terns in the former salt production ponds of South San Francisco Bay, California.

Table 15. The ranking of candidate models at the pond scale for foraging gulls in the former salt production ponds of South San Francisco Bay, California.

Table 16. The ranking of candidate models at the pond scale for roosting gulls in the former salt production ponds of South San Francisco Bay, California.

Table 17. The ranking of candidate models at the pond scale for foraging eared grebes in the former salt production ponds of South San Francisco Bay, California.....

Table 18. The ranking of candidate models at the pond scale for roosting eared grebes in the former salt production ponds of South San Francisco Bay, California.

Table 19. The ranking of candidate models at the pond scale for foraging northern shovelers in the former salt production ponds of South San Francisco Bay, California.

Table 20. The ranking of candidate models at the grid scale for roosting northern shovelers in the former salt production ponds of South San Francisco Bay, California.

Table 21. The ranking of candidate models at the pond scale for foraging and roosting ruddy ducks in the former salt production ponds of South San Francisco Bay, California

Table 22. The ranking of candidate models at the pond scale for foraging American avocets in the former salt production ponds of South San Francisco Bay, California 
Table 23. The ranking of candidate models at the pond scale for roosting American avocets in the former salt production ponds of South San Francisco Bay, California.

Table 24. The ranking of candidate models at the pond scale for foraging and roosting eared grebes in the active salt production ponds of South San Francisco Bay, California

Table 25. Variable importance values for significant model-averaged coefficients describing foraging and roosting waterbird guild abundance at the pond scale, in restoration ponds in South San Francisco Bay, California

Table 26. Optimal habitat characteristic values for waterbird guilds at the pond scale, as determined from General Additive Models, in restoration ponds, South San Francisco Bay, California

Table 27. Variable importance values for significant model-averaged coefficients describing foraging and roosting waterbird abundance at the pond scale in restoration ponds, South San Francisco Bay, California 126

Table 28. Optimal habitat characteristic values for waterbird species at the pond scale, as determined from General Additive Models, in restoration ponds, South San Francisco Bay, California.

Table 29. The ranking of candidate models at the grid scale for foraging and roosting dabbling ducks in the former salt production ponds of South San Francisco Bay, California

Table 30. The ranking of candidate models at the grid scale for foraging and roosting diving ducks in the former salt production ponds of South San Francisco Bay, California

Table 31. The ranking of candidate models at the grid scale for foraging and roosting small shorebirds in the former salt production ponds of South San Francisco Bay, California

Table 32. The ranking of candidate models at the grid scale for foraging and roosting medium shorebirds in the former salt production ponds of South San Francisco Bay, California

Table 33. The ranking of candidate models at the grid scale for foraging and roosting waders in the former salt production ponds of South San Francisco Bay, California.

Table 34. The ranking of candidate models at the grid scale for foraging and roosting piscivores in the former salt production ponds of South San Francisco Bay, California

Table 35. The ranking of candidate models at the grid scale for foraging and roosting terns in the former salt production ponds of South San Francisco Bay, California

Table 36. The ranking of candidate models at the grid scale for foraging and roosting gulls in the former salt production ponds of South San Francisco Bay, California

Table 37. The ranking of candidate models at the grid scale for foraging and roosting eared grebes in the former salt production ponds of South San Francisco Bay, California

Table 38. The ranking of candidate models at the grid scale for foraging and roosting northern shovelers in the former salt production ponds of South San Francisco Bay, California

Table 39. The ranking of candidate models at the grid scale for foraging and roosting ruddy ducks in the former salt production ponds of South San Francisco Bay, California

Table 40. The ranking of candidate models at the grid scale for foraging and roosting American avocets in the former salt production ponds of South San Francisco Bay, California

Table 41. Variable importance values for significant model-averaged coefficients describing foraging and roosting waterbird guild abundance at the grid scale in restoration ponds, South San Francisco Bay,

California

Table 42. Optimal habitat characteristic values for waterbird guilds at the grid scale, as determined from General Additive Models (GAMs), in restoration ponds, South San Francisco Bay, California

Table 43. Variable importance values for significant model-averaged coefficients describing foraging and roosting waterbird abundance at the grid scale in restoration ponds, South San Francisco Bay, California.....

Table 44. Optimal habitat characteristic values for waterbird species at the grid scale, as determined from General Additive Models (GAMs), in restoration ponds, South San Francisco Bay, California. 


\section{Conversion Factors}

U.S. customary units to International System of Units

\begin{tabular}{|c|c|c|}
\hline Multiply & By & To obtain \\
\hline \multicolumn{3}{|c|}{ Area } \\
\hline acre & 4,047 & square meter $\left(\mathrm{m}^{2}\right)$ \\
\hline acre & 0.4047 & hectare (ha) \\
\hline acre & 0.4047 & square hectometer $\left(\mathrm{hm}^{2}\right)$ \\
\hline acre & 0.004047 & square kilometer $\left(\mathrm{km}^{2}\right)$ \\
\hline \multicolumn{3}{|c|}{ International System of Units to U.S. customary units } \\
\hline Multiply & By & To obtain \\
\hline \multicolumn{3}{|c|}{ Length } \\
\hline centimeter $(\mathrm{cm})$ & 0.3937 & inch (in.) \\
\hline meter $(\mathrm{m})$ & 3.281 & foot $(\mathrm{ft})$ \\
\hline kilometer $(\mathrm{km})$ & 0.6214 & mile (mi) \\
\hline meter $(\mathrm{m})$ & 1.094 & yard (yd) \\
\hline \multicolumn{3}{|c|}{ Area } \\
\hline hectare (ha) & 2.471 & acre \\
\hline hectare (ha) & 0.003861 & square mile $\left(\mathrm{mi}^{2}\right)$ \\
\hline square kilometer $\left(\mathrm{km}^{2}\right)$ & 0.3861 & square mile $\left(\mathrm{mi}^{2}\right)$ \\
\hline square kilometer $\left(\mathrm{km}^{2}\right)$ & 247.1 & acre \\
\hline \multicolumn{3}{|c|}{ Volume } \\
\hline liter (L) & 0.264172 & gallon (gal) \\
\hline milliter $(\mathrm{mL})$ & 0.033814 & ounce, fluid (fl. oz) \\
\hline
\end{tabular}

\section{Abbreviations}

$\begin{array}{ll}\text { AIC } & \text { Akaike's Information Criterion } \\ \triangle A I C & \text { difference in AIC models } \\ \text { CDFW } & \text { California Department of Fish and Wildlife } \\ \text { CI } & \text { confidence interval } \\ \text { DEM } & \text { digital elevation model } \\ \text { FWS } & \text { U.S. Fish and Wildlife Service } \\ \text { GAM } & \text { Generalized Additive Model } \\ \text { GLMM } & \text { generalized linear mixed model } \\ \text { ISP } & \text { Initial Stewardship Plan } \\ \text { Project } & \text { South Bay Salt Pond Restoration Project } \\ \text { SFB } & \text { San Francisco Bay } \\ \text { South Bay } & \text { South San Francisco Bay } \\ \text { AMAV } & \text { American avocet } \\ \text { EAGR } & \text { eared grebe } \\ \text { NSHO } & \text { northern shoveler } \\ \text { ppt } & \text { parts per thousand } \\ \text { RUDU } & \text { ruddy duck } \\ \text { USGS } & \text { U.S. Geological Survey } \\ \text { UTM } & \text { Universal Transverse Mercator } \\ \text { VIF } & \text { Variance Inflation Factors }\end{array}$




\section{Trends and Habitat Associations of Waterbirds Using the South Bay Salt Pond Restoration Project, San Francisco Bay, California}

By Susan E. W. De La Cruz¹, Lacy M. Smith¹, Stacy M. Moskal ${ }^{1}$, Cheryl Strong ${ }^{2}$, John Krause ${ }^{3}$, Yiwei Wang4 and John Y. Takekawa1

\section{Executive Summary}

The aim of the South Bay Salt Pond Restoration Project (hereinafter "Project") is to restore 5090 percent of former salt evaporation ponds to tidal marsh in San Francisco Bay (SFB). However, hundreds of thousands of waterbirds use these ponds over winter and during fall and spring migration. To ensure that existing waterbird populations are supported while tidal marsh is restored in the Project area, managers plan to enhance the habitat suitability of ponds by adding islands and berms to change pond topography, manipulating water salinity and depth, and selecting appropriate ponds to maintain for birds. To help inform these actions, we used 13 years of monthly (October-April) bird abundance data from Project ponds to (1) assess trends in waterbird abundance since the inception of the Project, and (2) evaluate which pond habitat characteristics were associated with highest abundances of different avian guilds and species. For comparison, we also evaluated waterbird abundance trends in active salt production ponds using 10 years of monthly survey data.

We assessed bird guild and species abundance trends through time, and created separate trend curves for Project and salt production ponds using data from every pond that was counted in a year. We divided abundance data into three seasons - fall (October-November), winter (December-February), and spring (March-April). We used the resulting curves to assess which periods had the highest bird abundance and to identify increasing or decreasing trends for each guild and species.

${ }^{1}$ U.S. Geological Survey.

${ }^{2}$ U.S. Fish and Wildlife Service.

${ }^{3}$ California Department of Fish and Wildlife.

${ }^{4}$ San Francisco Bay Bird Observatory. 
We evaluated habitat characteristics associated with Project waterbird abundance at two scales(1) across the landscape at the pond scale, and (2) within ponds at the survey grid scale. At the pond scale, we analyzed bird abundance in relation to the following habitat characteristics:

- Water depth,

- Water salinity,

- Pond area,

- Presence of one or more islands,

- Topographic relief,

- Distance to SFB and urban areas,

- Percentage of levees open to public access including hunting, and

- Whether or not the pond was breached for tidal marsh restoration.

For gulls, we also included distance to the Tri Cities Landfill. At the grid scale, we analyzed bird abundance in relation to the following habitat characteristics:

- Water depth,

- Presence of one or more islands,

- Topographic relief, and

- Distance to nearest levee and creek or slough.

We separately analyzed data on eight foraging guilds, including dabbling ducks, diving ducks, small shorebirds, medium shorebirds, waders, piscivores, terns, and gulls. We also examined specific species of management interest owing to their abundance (northern shoveler [Anas clypeata], ruddy duck [Oxyura jamaicensis], and American avocet [Recurvirostra americana]), or unique habitat requirements (eared grebe [Podiceps nigricollis] — a hypersaline specialist). Additionally, we analyzed eared grebe abundance in relation to habitat characteristics in a subset of active salt production ponds. We separated the abundance of each guild or species by foraging and roosting behavior to assess any potential differences in habitat use. We identified pond habitat characteristics related to peak abundances of different guilds or species at pond and grid scales. Our results support the following conclusions on waterbird trends over time and the influence of habitat characteristics on waterbird abundance:

1. Waterbird abundance peaked in the Project ponds during winter (December-February). Total winter waterbird abundance increased non-linearly over the study period, more than doubling from 98,151 $\pm 38,826$ (mean \pm 95 -percent confidence interval) in 2002, prior to any Project management actions, to $235,936 \pm 16,564$ in 2014, near the completion of Phase I restoration.

2. Dabbling and diving ducks and small shorebirds constituted the largest proportion ( 73 percent) of all waterbirds in the Project ponds from October through April. Gulls (11 percent) and medium shorebirds (10 percent) were the next most abundant guilds, whereas all other guilds were less than 3 percent each.

3. Winter dabbling duck abundance in Project ponds increased nearly two-fold from 2002 to 2006, before plateauing to current values. In fall and spring, the number of dabbling duck also increased through 2006, but has since fluctuated.

4. Diving ducks and small and medium shorebirds had similar abundance trends over the study time period, increasing in fall and winter during two periods-2002-2005 and 2008-2014. Small shorebird abundance in Project ponds typically was highest in spring, although there was no pronounced trend and spring abundance was highly variable. Medium shorebird spring abundance in Project ponds increased from 2002 to 2007 and has since remained stable. 
5. Abundances of individual guilds in salt production ponds were from 0.8 to 8.6 times lower than in Project ponds. Abundances of individual species in production ponds were from 2 to more than 900 times lower than in Project ponds. However, abundance trends were similar between the two pond types for most taxa except for small and medium shorebirds, which generally declined in salt production ponds during all seasons throughout the study period.

6. Water depth was a key habitat variable that influenced the abundance of most guilds and species at both the pond and grid scale. At the pond scale, bird abundances were highest at a range of depths, starting at $0 \mathrm{~m}$ for foraging and roosting small shorebirds to $1.49 \mathrm{~m}$ for roosting diving ducks, including ruddy ducks. At the grid scale, highest abundances of both roosting and foraging small and medium shorebirds were associated with 0 -m depths, whereas diving duck, piscivore, and gull abundances were highest in grids that ranged from 0.33 to $2.51 \mathrm{~m}$ in depth. In addition to water-level manipulation, increasing topography and islands within ponds may be one approach to meeting the depth needs of multiple species.

7. Foraging and roosting dabbling and diving ducks (including northern shoveler and ruddy duck), piscivores, terns, and waders were most abundant in ponds with relatively low salinity ( $\leq 33$ parts per thousand [ppt]), and maintaining low salinities may further benefit these species. Only foraging gulls and eared grebes had a positive relation with salinity and were most abundant in ponds with salinities of 109-124 ppt. Conversely, roosting gulls and eared grebes, as well as foraging and roosting small and medium shorebirds, showed no relation with salinity.

8. Foraging and roosting diving ducks (including ruddy duck), piscivores, terns, gulls, waders, and eared grebes were most abundant in the largest ponds $\left(1.0-2.5 \mathrm{~km}^{2}\right)$ included in the study. Maintaining ponds with large areas of open water $\left(>1.0 \mathrm{~km}^{2}\right)$ may benefit these guilds and species. Dabbling ducks (including northern shoveler) and small and medium shorebirds (including American avocets) were not influenced by pond size. As Project restorations progress and mature, additional work on the effect of pond area and proximity to other suitable habitat in the mosaic of tidal wetlands and ponds may be critical to determine habitat requirements for species that are not sensitive to pond area under current (2017) conditions.

9. Variation in pond bottom elevation (topography) had a greater influence on bird abundance at the grid scale than at the pond scale. At the pond scale, topography influenced roosting dabbling ducks (including northern shoveler) and medium shorebirds (including American avocet), all of which were most abundant when topography ranged from 0 to $-0.61 \mathrm{~m}$. At the grid scale, foraging dabbling ducks (including northern shoveler), small and medium shorebirds (including roosting American avocet), waders, and roosting gulls were most abundant in grids where topography varied widely from 0.12 to $1.27 \mathrm{~m}$. Foraging diving duck abundance was associated with grids that had minimal topography $(0.12 \mathrm{~m})$. Our grid-scale results suggest that increasing small-scale topography in shallow water Project ponds may increase foraging use by many of the guilds we studied. Experimental studies to evaluate the response of waterbirds to topographic manipulation may further inform management of this habitat feature.

10. At the pond scale, the presence of one or more islands supported higher abundances of all roosting birds except eared grebes, and most foraging birds except gulls, medium shorebirds (including American avocet), and eared grebes. At the grid scale, the presence of one or more islands supported a higher abundance of foraging medium shorebirds (including American avocet), waders, northern shovelers, ruddy ducks and all roosting birds except diving ducks. Our analyses suggest that islands may be as critical as other key pond characteristics such as water depth and salinity for maintaining foraging and roosting abundances of birds from multiple guilds. 
11. Pond distance to SFB had little influence on the abundance of most guilds and species. However, roosting and foraging diving ducks (including foraging ruddy ducks) were most abundant in ponds that were about 1-2 km from SFB. Roosting and foraging gull abundance increased with pond distance from SFB.

12. Ponds closer to landfills supported a higher abundance of roosting gulls; however, this relation leveled off at about $2.8 \mathrm{~km}$ away from landfills.

13. The percentage of pond levees open to hunters during the hunting season (November-January) influenced both foraging and roosting guilds and species. Foraging piscivores, roosting dabbling ducks, and roosting wader abundances were linearly related to this variable and were least abundant in ponds with 100 percent of levees open to hunters. In contrast, the abundance of foraging gulls and roosting terns increased as the percentage of levees open to hunting increased. Additional analyses that include the presence of hunting blinds may be warranted.

14. Public access (based on the percentage of pond levees open to public access other than hunting and not on actual use by the public) had no influence on the abundance of most guilds and species. However, foraging small shorebirds and roosting gulls were most abundant in ponds where 70 and 46 percent, respectively, of levees were open to the public. Additional studies that examine effects of frequency of public use may be warranted.

15. Pond distance to urban areas did not influence the abundance of any guild or species.

16. Breached ponds supported fewer diving ducks (including roosting ruddy ducks), piscivores, and foraging eared grebes compared to unbreached ponds. Abundance was positively related to breached ponds only for foraging small shorebirds. As Project restorations progress, future work to investigate temporal and spatial use of breached habitat by all guilds may inform management of post-breach transitional habitat to maximize waterbird abundances.

17. Grid distance to pond levee influenced the abundance of most guilds and species. Small and medium shorebirds, American avocets, piscivores, gulls, foraging terns, eared grebes, and foraging northern shovelers and ruddy ducks were most abundant in grids that were closest to levees (0.0-0.25 km away), whereas roosting terns were most abundant in grids that were $0.3 \mathrm{~km}$ from levees. Research to determine which levee features attract each guild may be informative.

18. Grid distance to nearest creek or slough did not influence most guilds and species. Foraging small shorebirds and roosting medium shorebirds (including American avocets) were most abundant in grids closest to creek and slough features $(0.04 \mathrm{~km})$. In contrast, roosting piscivores were most abundant in grids far from creeks or sloughs $(0.95 \mathrm{~km})$.

19. Abundance of foraging eared grebes in salt production ponds was not related to any variable we evaluated; however, as with our findings in Project ponds, roosting eared grebes were most abundant in large ponds with increasing staff gauge values (a proxy for depth).

20. Eared grebes are hypersaline specialists with unique depth and salinity requirements that often do not overlap those of other guilds and species. Additional targeted studies on movements and seasonal habitat use of eared grebes may inform management opportunities for this species.

21. We summarize significant habitat characteristics identified in our analyses and compare their importance across guilds and species. Our results suggest that key habitat characteristics can be manipulated by managers to benefit one or more taxa. In many cases, the optimal habitat values overlap among guilds and species, providing the opportunity to manage individual or groups of ponds to benefit several species. 


\section{Introduction}

San Francisco Bay (SFB) is the largest estuary on the west coast of North America, supporting large populations of humans and wildlife. This estuary supports more than one-half million wintering and migratory shorebirds (Page, Stenzel and Kjelmyr, 1999; Morrison, 2001), and has been designated a Western Hemisphere Shorebird Reserve Network site because of its status as one of the most important staging and wintering areas on the Pacific Flyway. San Francisco Bay also supports as much as 44 percent of wintering diving duck populations observed in the Lower Pacific Flyway during U.S. Fish and Wildlife Service (FWS) mid-winter surveys (Richmond and others,. 2014; Trost, 2009). However, the SFB estuary has lost more than 80 percent of its historical tidal marsh habitat to urbanization, agriculture, and salt production (Goals Project, 1999). This includes about 14,000 ha (35,000 acres) that were converted to artificial salt evaporation ponds beginning in the 1850s (Goals Project, 1999). Although this conversion represented a major loss of tidal wetlands, the salt ponds are now used extensively as foraging and roosting habitat by migratory and resident waterbirds (Warnock and others, 2002). For example, during one spring migration, shorebird abundance within a single salt pond exceeded 200,000 birds (Stenzel and Page, 1988).

In a 2003 purchase agreement, 53 former salt evaporation ponds comprising 6,110 ha in the Alviso, Eden Landing, and Ravenswood salt production complexes of South San Francisco Bay (South Bay) were purchased from the salt production company Cargill, Inc. and transferred to the FWS and California Department of Fish and Wildlife (CDFW) for management. The South Bay Salt Pond Restoration Project (hereinafter "Project") is being conducted by a broad coalition of partners, including FWS, CDFW, the California Coastal Conservancy, and several other agencies (see http://www.southbayrestoration.org). These groups are working together to implement a large-scale, multi-phased plan to convert 50-90 percent of Project ponds to tidal marsh within the next 50 years (Goals Project, 1999; Steere and Schaefer, 2001; Siegel and Bachand, 2002; Stallings, 2004). One of the goals of the Project is to restore and enhance tidal marsh habitat for endemic species while maintaining and managing ponds to sustain migratory and resident waterbird populations.

Under the Initial Stewardship Plan (ISP 2006-2009; Stallings, 2004), water control structures were installed within the ponds to promote circulation of SFB water and to prevent salt accumulation. Phase I (2010-2015), the first component of the Project, included breaching three ponds to restore tidal flow for marsh restoration, augmenting two ponds with islands for roosting and nesting shorebirds, and adjusting water levels in other ponds to improve habitat for waterfowl and shorebirds. In Phase II, which began in 2016, the proposed restoration actions include breaching additional ponds and improvements to other pond habitat by adding features that support waterbird populations. Phase II and future restoration action alternatives are dependent on following adaptive management principles incorporating scientific findings to ensure that migratory waterbird populations are conserved as tidal marsh restoration moves forward. 
To assess the effects of management actions under the Project Final Environmental Impact Statement (EDAW and others, 2007), the U.S. Geological Survey (USGS) began monitoring waterbird abundance and distribution as well as water-quality parameters in the Project ponds in 2002 . This early monitoring work was initiated with support of the USGS, FWS, California Coastal Conservancy, $\mathrm{CDFW}$, and non-profit foundations to provide the Project with data to inform future restoration decisions. Data collected during the ISP period and Phase I provide an unparalleled opportunity to assess the effects of restoration actions and the potential for adaptive management to sustain waterbird populations. As the Project proceeds, there are several key uncertainties about how to enhance and manage existing ponds to maximize waterbird populations as the overall pond area is reduced. Whereas previous research has highlighted the importance of island habitat within ponds for wintering and breeding waterbirds (Ackerman and others, 2014), manipulation of additional pond characteristics also may improve habitat and increase waterbird abundance. Herein, we evaluate relations between waterbird abundance and multiple pond characteristics to help inform future management actions designed to maximize waterbird habitat in managed ponds.

\section{Research Questions}

The overarching objectives of our study were to evaluate waterbird abundance trends and to assess habitat characteristics that support high abundances of waterbirds within the former salt ponds of the South Bay. Specifically, we addressed the following research questions:

1. How have waterbird abundances changed within the Project and active salt production ponds since the inception of the phased restoration process?

2. How do pond spatial location and habitat characteristics influence waterbird abundance across Project ponds?

3. How do pond habitat characteristics influence waterbird abundance within Project ponds?

4. How do pond characteristics influence eared grebe abundance across active salt production ponds in the South Bay?

\section{Study Area}

Our study area includes former and current salt production ponds in South Bay, California $\left(37.42-37.62^{\circ} \mathrm{N} ; 121.93-122.22^{\circ} \mathrm{W}\right.$; fig. 1), which are divided into five complexes: (1) Eden Landing ( $\mathrm{N}=28)$, (2) Alviso ( $\mathrm{N}=25)$, (3) Ravenswood ( $\mathrm{N}=10)$, (4) Mowry ( $\mathrm{N}=6)$, and (5) Newark ( $\mathrm{N}=16)$. Ponds in the Eden Landing, Alviso, and Ravenswood complexes are part of the restoration Project. Eden Landing is managed by the CDFW as part of the Eden Landing Ecological Reserve, and Alviso and Ravenswood are both managed by the FWS as part of the Don Edwards San Francisco Bay National Wildlife Refuge. Cargill, Inc. manages the Mowry and Newark pond complexes for salt production. Ponds range in size from 0.1 to $2.8 \mathrm{~km}^{2}$ and vary in depth, salinity, and spatial proximity to SFB (table 1). 


\section{Methods}

\section{Waterbird Surveys}

From October 2002 through April 2013, USGS conducted monthly waterbird counts in Eden Landing, Alviso, and Ravenswood. San Francisco Bay Bird Observatory counted waterbirds every 6 weeks from January 2014 to April 2015 in Alviso, Eden Landing, and Ravenswood, and monthly from October 2005 to April 2015 in Mowry and Newark. We counted waterbirds at high tide when adjacent mudflats were inundated and shorebird abundances within ponds were likely at their peak (Warnock and others, 2002). We overlaid pond maps with $250 \times 250-\mathrm{m}$ (6.25-ha) Universal Transverse Mercator (UTM) grids and recorded species and abundance by grid to document the spatial distribution of birds within ponds. We counted all birds using spotting scopes and binoculars either on foot or from a vehicle along pond levees. We recorded each bird as foraging or roosting based on behavior at the time of observation. We assigned species observed (table 2) to foraging guilds (listed as follows with example species):

1. Dabbling ducks (dabblers) — northern shoveler (Anas clypeata) and American wigeon (A. americana),

2. Diving ducks — ruddy duck (Oxyura jamaicensis),

3. Piscivores-double-crested cormorant (Phalacrocorax auritus) and American white pelican (Pelecanus erythrorhynchos);

4. Gulls_-ring-billed gull (Larus delawarensis);

5. Terns-Forster's tern (Sterna forsteri);

6. Waders - great egret (Ardea alba);

7. Medium shorebirds-marbled godwit (Limosa fedoa) and willet (Tringa semipalmata), and

8. Small shorebirds - western sandpiper (Calidris mauri), dunlin (C. alpina), and long-billed dowitcher (Limnodromus scolopaceus).

Based on Project goals (ISP 2006-2009; Stallings, 2004), we also identified four species of interest (American avocet, Recurvirostra americana; eared grebe, Podiceps nigricollis; northern shoveler; and ruddy duck) to evaluate individually. Except for eared grebes, individual species also were included in their respective guild for analysis.

\section{Environmental Data}

We collected monthly salinity readings from each surveyed pond using a Hydrolab Mini Sonde ${ }^{\circledR}$ (Hydrolab-Hach Company, Loveland, Colorado) or, if salinity exceeded the maximum value measured by the instrument, we measured specific gravity with a hydrometer (Ertco, West Paterson, New Jersey) and converted to salinity with the 1978 Practical Salinty Scale (Lewis, 1980). Each month, we sampled the same 1-5 locations in each pond and averaged the result across all locations within the pond.

We obtained pond elevation data for 35 Project ponds from bathymetric surveys conducted during $2003-04$ (Athearn and others, 2010). For 19 Project ponds lacking bathymetric data, we used lidar data obtained when ponds were exposed (dewatered), and for 2 Project ponds with partial bathymetry, we used a combination of lidar and bathymetry (Athearn and others, 2010). We interpolated 
bathymetric and lidar elevation data using Inverse Distance Weighting in ArcMap ${ }^{\mathrm{TM}} 9.1$ (Spatial Analyst, ArcGIS ${ }^{\mathrm{TM}}$ 9.1, ESRI, Redlands, California) to create digital elevation models (DEMs) with 25 $\mathrm{m}$ resolution for each pond. Within the ponds, there is a $0-2 \mathrm{~cm}$ difference between the lidar and bathymetric DEMs (Athearn and others, 2010). During each survey, we recorded water height within a pond by reading staff gauges of known elevation installed within each pond. We calculated water depth by taking the difference between staff gauge readings and pond elevation data (NAVD 88 vertical datum). We defined pond and grid topography as the standard deviation of the mean elevation (for example, large deviations meant greater topographic relief on the pond bottom). We did not include elevations of islands or levees in our calculation of topography.

We used ArcGIS ${ }^{\mathrm{TM}} 10.1$ (ESRI, Redlands, California) to derive spatial pond attributes. We used 2009 National Agricultural Imagery Program (1-m resolution) aerial imagery to digitize islands within ponds in $\operatorname{ArcMap}^{\mathrm{TM}}$ 10.1. We modified island outlines using ground-truthed, real-time kinematic Global Positioning System (Leica Geosystems Inc., Norcross, Georgia) survey points. We defined an island as land that was surrounded by water and exposed year-round. Our analysis included 20 ponds with islands, and the number of islands per pond ranged from 1 to 22 . We calculated the number of islands that intersected each bird survey grid (minimum=0, maximum=9). We used the center point of each pond to calculate the distance between ponds and SFB, urban areas, and landfills. We used the center point of each grid to calculate the distance between grids and levees, adjacent creeks, and sloughs. We obtained information on the timing and percentage of levees open to public access, including hunting, from State and Federal maps and discussions with area managers. The percentage of levees open to hunting was considered zero during non-hunting months (February-October). We considered the four units of Ravenswood Pond RSF2 (fig. 1) as separate ponds because each unit has water control structures that allow for individual management.

\section{Statistical Analysis}

We assessed bird guild and species abundance trends from October 2002 through April 2015 using non-parametric locally weighted scatterplot smoothing (LOESS) regression in R (package ggplot2). We created separate trend curves for Project and salt production ponds using data from every pond counted in a year. We divided abundance data into three seasons - fall (October-November), winter (December-February), and spring (March-April). We excluded September and May count data from our analyses because differences in the timing of migration among species introduced variability in the abundance data that obscured seasonal abundance patterns which were the focus of this study. While understanding arrival and departure times of migratory waterbirds is an important aspect of their ecology, addressing migration phenology was not within the scope of this study. We used the resulting LOESS curves to assess which periods had the highest bird abundance and to identify increasing or decreasing trends for each guild and species.

We separately modeled foraging and roosting abundances of waterbird guilds and species using generalized linear mixed models (GLMMs) with a negative binomial distribution to account for the large number of zero observations in our dataset (Warton, 2005). We divided our analyses into two scales - pond and grid, - to evaluate the influence of predictor variables on abundance at landscape and within-pond levels. 
In the first stage of our analysis, we examined variables that may influence bird abundance at the pond scale. We tested all variables for collinearity using Variance Inflation Factors (VIF; R package car; Zuur and others, 2009). We excluded pond perimeter because it was highly correlated with pond area; however, the VIF for the remaining variable pairs ranged from 1.07 to 2.28 and were below the threshold of 3.0 recommended for exclusion (Zuur and others, 2009). We built a candidate set of models that included all possible combinations of the following variables:

- Water depth,

- Water salinity,

- Pond area,

- Pond distance to SFB and urban areas,

- Pond management type,

- Presence of one or more islands,

- Variation in pond topography, and

- Percentage of the pond levees open to the public or to waterfowl hunting (table 1).

We did not have complete information on the location or number of hunting blinds in all ponds; thus, we only accounted for hunting from levees and did not account for hunting access using blinds. The total number of models in each candidate set was 1,024. We built a separate set of 2,048 candidate models for gulls, which included distance to the Tri-Cities Landfill (Fremont, California; UTM 10S 589251.34, E 4149803.26) in addition to the listed variables. We considered two pond management types - ponds with breached levees for tidal marsh restoration and un-breached ponds. Nine ponds were breached during our study period and were classified as breached only during the post-breach period. We included pond area in the model to assess the influence of area on abundance. We might expect to find higher bird abundances in larger ponds because the ponds can accommodate more birds; however, we wanted to test this assumption, as contrary results could have important implications for management of birds. We excluded data collected during identified construction periods in a given pond.

In the second stage of our analysis, we evaluated the influence of habitat variables on bird abundance within ponds at the grid scale. We assessed all variable pairs for collinearity and determined that VIF ranged from 1.00 to 1.34 for all combinations. We developed a candidate set of models that included all possible combinations of the following variables:

- Grid water depth,

- Grid distance to the pond levee and to nearest creek or slough,

- Variation in grid topography (calculated as the standard deviation of grid elevation), and

- Presence of island(s) in grid.

Grid area was an artifact of our survey design method and we were not interested in assessing its effect; however, we included this variable in every model to account for potential minor differences in grid size at the edges of ponds.

An assumption of our GLMM analyses is that bird abundance has a log-linear relation with the independent habitat variables. We included pond, grid (grid-scale analysis), month, and year as random effects in each model to account for potential sources of additional dispersion and because we were not interested in assessing temporal differences. We used the glmmADMB package in R statistical software to fit all models (Fournier and others, 2012; Skaug and others, 2012; R Core Team, 2014). As a reference for comparison, we included null models in each of our pond-scale (intercept only) and gridscale (intercept + grid area) analyses. We ranked models with Akaike's Information Criterion (AIC) using a customized package (ModelInference package in R, M. Herzog, unpublished package) based on the information-theoretic model selection paradigm (Burnham and Anderson, 2002). 
We calculated model-averaged parameter estimates across all models and assessed variable importance (sum of the weights of all models containing that variable; Burnham and Anderson, 2002) to determine the effect of each parameter on bird abundance. We graphed and evaluated the 95-percent confidence interval $(\mathrm{CI})$ of each model-averaged parameter estimate and considered variables significant only if the CI did not overlap zero.

To examine relations between model-averaged predictor variables and response variables, we used Generalized Additive Models (GAMs, gamlss package in R; Tavares and others, 2015). These models have the flexibility to model discrete statistical distributions (for example, negative binomial) commonly associated with abundance data as well as nonlinear effects of covariates on the predicted mean (Hastie and Tibshirani, 1990). For each guild and species, we built GAMs using only the variables determined to be significant in the GLMM analysis. This included variables that had variable importance values greater than or equal to $(\geq) 0.92$ and model-averaged parameter estimates with 95percent CIs that did not overlap zero. We graphed abundance as a function of each habitat variable and held all other variables in the model at a mean value. Month and year were included as random effects, and we used a cubic spline smoother in all models. We assessed GAM plots to determine optimal values of significant habitat variables for a given guild or species at the pond and grid scale. For a few guilds and species (roosting and foraging piscivores, roosting terns, roosting eared grebe, and foraging ruddy duck), grid-scale data were too patchily distributed to find a solution using GAMs that contained all significant variables. For these taxa only, we ran GAMs on each variable individually; thus, the resulting plots represent bird abundance as a function of a single habitat variable and do not account for the effect of other variables determined to be significant in GLMM models. Binomial variables, such as island presence or absence, could only be included in models as linear variables and, therefore, are not shown in GAM plots.

In addition to modeling eared grebe abundance in Project ponds, we developed a separate analysis for eared grebes using the active salt production ponds in Mowry and Newark, where eared grebe abundance historically has been highest (Athearn and others, 2012). Pond elevation data were not available for the salt production ponds, so we used staff gauge value as a relative approximation for depth. We assumed that staff gauges were installed to the same elevation datum across all ponds and were comparable to each other. Island data were not available for analyses. Thus, our analyses of eared grebes in salt production ponds included the following variables:

- Salinity,

- Staff gauge value,

- Pond area, and

- Pond distance to SFB and to urban areas.

We restricted our analyses to the pond scale because of limited grid-scale data in salt production ponds. Following the same methods used for Project ponds, we modeled all possible combinations of variables using a GLMM with a negative binomial distribution. The total number of models in our candidate set was 32 . 


\section{Results}

\section{Abundance Trends}

We conducted 5,055 Project pond surveys during 13 field seasons from fall 2002 through spring 2015 and observed 101 species of waterbirds (table 2). In general, the waterfowl (dabbling and diving ducks, 17 percent each), shorebird (small shorebirds, 39 percent; medium shorebirds, 10 percent), and gull (11 percent) guilds represented the most abundant bird taxa in Project ponds across all years of the study, whereas the other guilds (waders, terns, piscivores) represented less than $(<) 3$ percent each. Overall, peak waterbird abundances occurred in the Project area during winter (December-February). Total winter waterbird abundance increased non-linearly over the study period, more than doubling from 98,340 $\pm 38,761$ (mean \pm 95 -percent confidence interval) in 2002, prior to the ISP period, to $235,936 \pm 16,564$ in 2014, near the completion of Phase I.

We conducted 1,399 surveys of active salt production ponds (Mowry and Newark complexes) during nine field seasons from fall 2005 through spring 2015, and observed 79 species of waterbirds (table 2). Waterfowl (dabbling ducks, 11 percent; diving ducks, 12 percent), shorebirds (small shorebirds, 28 percent; medium shorebirds, 12 percent), gulls (23 percent), and eared grebes (12 percent) were the most abundant bird taxa in salt production ponds across all years of the study, whereas the other guilds (piscivores, waders, terns) represented $<3$ percent each. Overall, yearly peak waterbird abundances occurred during winter (December-February), with little change in mean abundance between winters of $2005(47,730 \pm 16,867)$ and $2014(43,033 \pm 5570)$.

Across guilds and species, abundance was variable by season and year. For example, dabbling duck abundance was highest during winter in Project ponds and increased more than three-fold between 2002 and 2006, before plateauing to current mean values of just less than 50,000 birds (fig. 2). In fall and spring, dabbling duck numbers also increased through 2006, but have since fluctuated (fig. 2). Diving duck abundance was highest during winter and increased within the Project ponds during two periods - 2002-2005 and 2008-2014 (fig. 3). Overall, abundances of diving ducks in fall and winter have doubled on Project ponds since surveys began in 2002, whereas spring abundances have remained similar, albeit with large confidence intervals (fig. 3). Diving duck abundance trends within active salt production ponds also are increasing during winter and have remained stable during fall and spring (fig. 3).

Small and medium shorebird abundance trends in the Project ponds were similar to trends of diving ducks, showing increases in fall and winter during two periods, 2002-2005 and 2008-2014 (figs. 4 and 5). Small shorebird abundances in Project ponds typically were highest in spring; however, spring numbers were variable with large 95-percent CIs and a flat trendline since 2002 (fig 4). Medium shorebird spring abundances in Project ponds increased from 2002 to 2007 and have since remained fairly stable (fig. 5). In contrast, small and medium shorebird abundances in the salt production ponds generally have decreased across all seasons (figs. 4 and 5).

The highest abundance of waders occurred during fall in Project ponds; their population increased from 2002 to 2005, decreased from 2006 to 2008, and then remained stable (fig. 6). Wader abundance in Project Ponds during winter and spring increased from 2002 to 2006 and from 2011 to 2014 (fig. 6). Wader population trends in the salt production ponds generally followed the trends in the Project ponds (fig. 6).

The highest abundance of piscivores occurred during fall in Project and salt production ponds (fig. 7). Within Project ponds, their fall abundance increased from 2002 to 2005 and from 2008 to 2012, whereas in salt production ponds their abundance has continued to increase (fig. 7). There was a gradual increase in piscivore abundance during winter and spring in Project and salt production ponds (fig. 7). 
Tern abundance trends were similar to piscivore abundance trends, except that their abundance in salt production ponds during fall decreased from 2010 to 2014 and there was a sharper increase in abundance in Project ponds during spring from 2011 to 2014 (fig. 8).

Gull abundance reached its peak in Project ponds during winter 2004-06 and in salt production ponds during fall and winter 2007-08, after which the population decreased (fig. 9). During winter and spring 2013 and 2014, the winter and spring gull abundance in Project ponds began increasing, but remained lower than the peak abundances (fig. 9).

The highest abundance of eared grebes occurred in salt production ponds during spring (fig. 10). Eared grebe abundance decreased in the Project ponds from 2002 to 2006, and in the salt production ponds from 2005 to 2008 (fig. 10). The population began increasing in the Project ponds in 2011 and in the salt production ponds after 2008 (fig. 10).

The highest abundance of northern shoveler and ruddy ducks occurred in Project ponds during fall and winter (figs. 11 and 12). Northern shoveler abundance in these ponds increased consistently during winter and fluctuated during fall and spring (fig. 11). Ruddy duck abundance in Project ponds increased consistently during fall, increased during two periods in winter (2002-2005 and 2008-2014), and remained stable in spring from 2002 to 2008 before slightly increasing from 2008 to 2014 (fig. 12).

The highest abundance of American avocets occurred in Project ponds during fall and winter, increasing from 2002 to 2006 and from 2010 to 2014 (fig. 13). Their abundance in salt production ponds decreased in winter and spring and followed the same trend as in the Project ponds during fall (fig. 13).

\section{Pond-Scale Models}

In any single year, only a subset of the ponds had a complete set of associated environmental data; thus, the average number of ponds included in the pond-scale dataset each year was 43 (range 3748), excluding fall 2002, in which we only had data for seven ponds (table 1). We modeled foraging and roosting abundance of seven guilds and four species of interest, for a total of 22 separate analyses (tables 3-24). Variable importance values for all significant pond-scale, model-averaged coefficients for guilds were $\geq 0.93$ (table 25 ).

Overall, our model results indicated that the influence of pond characteristics varied by guild and species, and to a lesser degree by foraging and roosting behavior. Model-averaged GLMM coefficients and GAM-derived relations between bird abundance and pond depth showed that ponds with deeper water (0.4-1.5 $\mathrm{m}$ in depth; figs. 14 and 15, tables 25 and 26) supported the highest abundances of foraging and roosting diving ducks, piscivores, and foraging gulls. Ponds with shallower water (0-0.4 m; figs. 14 and 15, tables 25 and 26) supported the highest abundances of foraging and roosting small and medium shorebirds, foraging waders, and roosting gulls.

Pond salinity was a significant parameter for several guilds (fig. 16). Foraging and roosting dabbling and diving ducks, piscivores, terns, and waders were present in highest abundances in low salinity (<17 ppt) ponds, whereas foraging gull abundances peaked at high salinities (124 ppt; fig. 17; tables 25 and 26). For many guilds (foraging and roosting waders, as well as foraging piscivores, terns and gulls), abundance increased with pond area, suggesting that there was not an optimal pond size within the range of ponds we evaluated (figs. 18 and 19). However, foraging and roosting diving duck abundance leveled off in ponds that were about $1.25 \mathrm{~km}^{2}$ (125 ha), roosting terns in ponds that were $>1.5 \mathrm{~km}^{2}$ (150 ha), and roosting gulls in ponds $>1.75 \mathrm{~km}^{2}$ (175 ha; fig. $19 \mathrm{~A}-\mathrm{B}, \mathrm{H}$, and J; table 26).

Responses to landscape features, such as topography and distance to SFB, varied among guilds. Increased pond topography (variation in pond bottom elevation) was important to some roosting guilds, but did not have a significant effect on foraging abundances of any guild (fig. 20). Roosting dabbling duck abundance was highest at the maximum pond topography value, $0.61 \mathrm{~m}$ (fig. 21 A; table 26), whereas roosting medium shorebird abundances peaked in ponds with topography values $\geq 0.15 \mathrm{~m}$ (fig. 
21B; table 26). Ponds with at least one island (compared to ponds with no islands) supported higher abundances of all roosting guilds as well as of foraging dabbling and diving ducks, piscivores, terns, and waders (fig. 22). Ponds within $1.1 \mathrm{~km}$ of SFB were optimal for roosting and foraging diving ducks, whereas gull abundances increased linearly in ponds that were $>0.9 \mathrm{~km}$ from SFB (figs. 23 and 24; table 26). Roosting gull abundance was highest in ponds adjacent $(2.8 \mathrm{~km})$ to landfill, decreased linearly as distance from landfill increased, but leveled off in ponds $\geq 14 \mathrm{~km}$ from the landfill (fig. 25; table 26).

The proportion of levees open to the public for hunting or other access also influenced the abundance of several guilds. Foraging gull and roosting tern abundances increased linearly with increasing percentages of levees open to hunting, whereas roosting dabbling ducks and waders and foraging piscivores abundances had negative linear relations with percentage of levees hunted (figs. 26 and $27 \mathrm{~A}-\mathrm{E}$; table 26). Ponds with a higher proportion of levees open to public access (other than hunting), supported higher abundances of foraging small shorebirds (fig. 28). Foraging small shorebird abundance had a sinusoidal relation with percentage of levees open to the public, with highest abundances in ponds that had 0 and 70 percent of their levees open, whereas their lowest abundances occurred in ponds with 20-40 percent of their levees open (fig. 29A; table 26). In contrast, roosting gulls reached their peak abundance in ponds with about 46 percent of their levees open to the public (fig. 29B; table 26).

There was no relation between pond distance to urban areas and the abundance of any guild (fig. 30). Pond management type did influence abundance; breached ponds supported a higher abundance of foraging and roosting small shorebirds and a lower abundance of foraging and roosting diving ducks and piscivores relative to unbreached ponds (fig. 31).

Specific species generally followed the trend of their associated guilds. For example, like the medium shorebird guild, foraging and roosting American avocet abundance was highest in shallow ponds (0.30-0.32 m; figs. 14 and 32, tables 27 and 28) with one or more islands (figs. 20 and 22, table 27). Roosting avocet abundance also increased with pond topography, peaking at $0.25 \mathrm{~m}$ (fig. $32 \mathrm{C}$; tables 27 and 28).

Ponds with low salinity (8.0 ppt; figs. 17, 33A-B; tables 27 and 28) and one or more islands (fig. 22, tables 27 and 28) supported higher abundances of foraging and roosting northern shoveler. Abundances of roosting northern shovelers increased non-linearly as pond topography increased, and peaked at the maximum value of $0.61 \mathrm{~m}$ (fig. 20, fig. 33D; tables 27 and 28). Northern shoveler roosting abundances were negatively associated with the percentage of levees open to hunting, peaking at $0 \%$ (fig. 26, fig. 33C; tables 27 and 28).

Ponds with lower salinity, deeper water, one or more islands, larger area, and closer to SFB relative to other ponds supported higher abundances of foraging and roosting ruddy ducks (figs. 14, 16, 18, 22-23; table 27). Unbreached ponds supported a higher abundance of roosting ruddy ducks, but did not have a significant influence on foraging ruddy ducks (fig. 31, table 27). Foraging and roosting ruddy ducks reached peak abundances at about 11 and 7 ppt salinity, respectively (fig. 34C-D; table 28). Optimal depth for foraging ruddy ducks was about $0.74 \mathrm{~m}$, whereas optimal depth for roosting ruddy ducks was $1.73 \mathrm{~m}$ (fig. 34A-B; table 28). Foraging ruddy duck abundance peaked when pond area was about $2.30 \mathrm{~km}^{2}$ (230 ha), ponds were about $1 \mathrm{~km}$ from the edge of SFB, and roosting abundance peaked in ponds $\geq 1.25 \mathrm{~km}^{2}$ (125 ha) that were less than $1.62 \mathrm{~km}$ from SFB (fig. $34 \mathrm{E}-\mathrm{G}$, table 28 ).

We assessed eared grebes within Project ponds, as well as separately within active salt production ponds at the pond scale. Within the Project ponds, those with deeper water or greater area supported higher abundances of foraging and roosting eared grebes (figs. 14 and 18; table 27). Unbreached ponds and those with higher salinity supported higher abundances of foraging eared grebes (figs. 16 and 31; table 27). Optimal depths for foraging and roosting eared grebes in Project ponds were $>0 \mathrm{~m}$ and $1.29 \mathrm{~m}$, respectively (fig. 35A-B; table 28). Foraging eared grebe abundances peaked in 
ponds with salinity around $109 \mathrm{ppt}$, and foraging and roosting grebe abundances increased with a pond area as large as $2.30 \mathrm{~km}^{2}$ (230 ha; fig. 35C-E; table 28). Within salt production ponds, the 95-percent confidence intervals of all model-averaged variables overlapped zero for foraging eared grebes, suggesting none of these variables influenced them (fig. 36). However, roosting eared grebes were more abundant in larger, deeper production ponds (fig. 36).

\section{Grid-Scale Models}

We modeled foraging and roosting abundance of nine guilds and three species of interest, for a total of 12 separate analyses at the grid-scale (tables 29-40). Model-averaged results from the GLMM analyses indicate that grid water depth was an important predictor variable for all guilds regardless of foraging or roosting behavior (fig. 37), whereas GAM results show great variability in depths used among guilds (fig. 38A-P). Grids with deeper water supported higher abundances of foraging and roosting diving ducks and roosting piscivores (fig. 37, table 41), with optimal depths for these groups ranging from 0.8 to $1.0 \mathrm{~m}$ (fig. 38C-D, K-L; table 42). Grids with deeper water also supported higher abundances of foraging terns $(0.57 \mathrm{~m})$ and gulls $(2.51 \mathrm{~m})$; however, roosting gulls and terns were associated with shallower grids $(0 \mathrm{~m}$ and $0.48 \mathrm{~m}$, respectively; fig. $38 \mathrm{I}-\mathrm{J}, \mathrm{M}-\mathrm{N}$; table 42$)$. All other guilds had a negative relation with depth (fig. 37), indicating a higher abundance of birds in grids with shallower water $(\leq 0.65 \mathrm{~m}$; fig. 38A-P, table 42$)$.

Other grid characteristics also predicted waterbird abundance. Grids with one or more islands supported higher abundances of foraging waders and medium shorebirds, as well as all roosting guilds except diving ducks (fig. 39; table 41). Grids located closer to levees supported highest abundances of foraging and roosting small and medium shorebirds $(0.05 \mathrm{~km})$, foraging $(0.05 \mathrm{~km})$ and roosting gulls $(<0.45 \mathrm{~km})$, foraging $(>0.05 \mathrm{~km})$ and roosting piscivores $(0.05 \mathrm{~km})$, waders $(0.08 \mathrm{~km}$ foraging and roosting), and foraging terns $(0.06 \mathrm{~km})$, and a lower abundance of roosting terns (peak abundance at $0.35 \mathrm{~km}$; figs. 40, $41 \mathrm{~A}-\mathrm{L}$; tables 41 and 42). Grids located closer to sloughs and creeks supported highest abundances of foraging small shorebirds $(0.04 \mathrm{~km})$ and roosting medium shorebirds $(0.04 \mathrm{~km})$, whereas grids farther from creeks and sloughs had highest abundances of roosting piscivores $(0.95 \mathrm{~km}$; figs. 42, 43A-B; tables 41 and 42). Grids with increased topography supported a higher abundance of foraging and roosting small shorebirds $(0.25 \mathrm{~m})$, foraging waders $(1.25 \mathrm{~m})$, roosting medium shorebirds $(1.10 \mathrm{~m})$ and gulls $(0.84 \mathrm{~m})$, and foraging dabbling ducks $(1.07 \mathrm{~m}$; figs. 44, 45A, D-I; tables 41 and 42$)$. However, grids with minimal topography supported highest abundances of foraging diving ducks $(0.12$ m; fig. 45B; tables 41 and 42).Species responses to grid characteristics were similar to their respective guild-level responses. Abundances of foraging and roosting American avocets were highest in shallow grids $(0.01-0.03 \mathrm{~m})$, closer to creeks $(0.4 \mathrm{~km})$ and levees $(0.06-0.07 \mathrm{~km})$, with one or more islands (figs. 37, 39, 40, 42, 46A-F; tables 43 and 44). Grids with increased topography $(>0.5 \mathrm{~m})$ also supported a higher abundance of roosting American avocets (figs. 44B, 46G, tables 43 and 44). Shallow grids $(0.19-0.42 \mathrm{~m})$ with one or more islands supported the highest abundance of foraging and roosting northern shovelers (figs. 37, 39, 47A-B, tables 43 and 44), and grids closer to levees (0.06 km; figs. 40, $47 \mathrm{C}$, tables 43 and 44$)$ and with greater topography $(1.03 \mathrm{~m}$; figs. 44A, 47D; tables 43 and 44$)$ supported highest abundances of foraging northern shovelers. Highest abundances of foraging and roosting ruddy ducks were in deep grids $(0.78-0.86 \mathrm{~m}$; fig. $48 \mathrm{~A}-\mathrm{B}, 43$ and 44$)$ that were situated close to levees $(0.05 \mathrm{~km}$; fig. $48 \mathrm{C}$, tables 43 and 44$)$ and contained one or more islands (table 43$)$. Grids closer to levees $(0.05 \mathrm{~km}$; figs. 40, 49C-D, tables 43 and 44$)$, with deeper water (1.5 m; figs. 37, 49A$\mathrm{B}$, tables 43 and 44) supported the highest abundances of foraging and roosting eared grebes. 


\section{Discussion}

The goal of our study was to provide science support to resource managers tasked with maintaining migratory bird populations in the Project. To understand waterbird response to active pond management and preliminary wetland restoration efforts in the Project area, we investigated trends in abundance over a 13-year period that encompassed the pre-restoration period (2002-06), the ISP (200610) and Phase I (2010-15), and we evaluated the effects of habitat characteristics on waterbird abundance. We assessed bird-habitat relations at two spatial scales to determine how pond location in the landscape, as well as conditions across and within ponds, influenced bird abundance.

\section{Abundance Trends}

We noted a net increase in overall waterbird abundance during winter in Project ponds, driven primarily by the waterfowl and shorebird guilds. For waterfowl, this pattern mirrors long-term (19892014) and short-term (2005-14) baywide trends showing significant increases in SFB estuary dabbling duck and weak increases in South Bay diving duck abundances, although elsewhere in SFB diving ducks are decreasing (San Francisco Estuary Partnership, 2015). Diving duck trends in the Project area can be attributed to ruddy ducks, which represented an average of 64 percent $( \pm 6$ percent standard deviation) of all diving ducks in the ponds across the study period. Analyses of SFB mid-winter survey data show that ruddy ducks have increased significantly in the South Bay in general, but that other diving ducks, such as Scaup (Aythya spp.), have decreased substantially (San Francisco Estuary Partnership, 2015). After a period of rapid increase, winter abundance of dabbling ducks in Project ponds leveled off in 2006 and the trend has remained flat. Given that dabbling duck numbers have been increasing in SFB (San Francisco Estuary Partnership 2015) overall, this flat trend may indicate Project ponds are reaching carrying capacity for this guild.

A comparison of shorebird counts conducted in November 1990-92 and 2006-08 across SFB showed that 14 of 22 shorebird species had stable or increasing populations between these time periods (Wood and others, 2010). The South Bay subregion, including all the Project ponds, held more than one-half of all shorebirds in SFB and had the least amount of change in shorebird abundances between the two time periods (Wood and others, 2010). Fall and winter shorebird trends observed in our study had similar stable to increasing patterns, and shorebird abundance has continued to increase in Project ponds since the conclusion of the Wood and others (2010) study in 2008.

Although we observed a net increase for most waterbirds over the study period, this trend was non-linear because of a conspicuous decrease for shorebirds and diving ducks starting in 2005 to 2006 and reversing in 2008. The cause of this decrease is not clear. The ISP period, during which pond water depth and salinity management was initiated, began in 2006 and could have contributed to this change. However, during the same period, we observed similar trends in managed ponds of the Napa-Sonoma Marsh in North San Francisco Bay (author's unpub. data, U.S. Geological Survey, accessed 15 September 2016), suggesting that Project pond management may not have been the cause. Other possible explanations for this temporary decrease include range-wide or regional shifts in distribution of these species owing to climatic conditions or management practices elsewhere. Based on 2006 waterfowl breeding population data, most North American species estimates were greater than their long-term average during the period of decrease in Project ponds, suggesting that continental population changes did not influence abundance (U.S. Fish and Wildlife Service, 2006). However, population estimates for Scaup were at record lows during this same period, and Bufflehead estimates were 51 percent lower than 2005 estimates (U.S. Fish and Wildlife Service, 2006), potentially having some effect on the diving duck numbers we observed in SFB overall. 


\section{Habitat Modeling}

Management of Project pond characteristics may have played a role in the increased abundance we observed for some waterbird guilds. For example, the primary changes to Project ponds under ISP and Phase I management actions during our study period included manipulating water depths (both increasing and decreasing), adding islands, and decreasing salinities, in part to maintain or enhance abundance of waterbirds representing multiple guilds (Stallings, 2004). We determined that water depth was a significant driver of abundance for nearly all guilds and species we studied, particularly at the grid scale. The influence of water depth on species occurrence, richness, and abundance has been shown in other systems, and many of our results were within range of those reported in other studies. For example, wading bird abundance and habitat selection in the Everglades is largely influenced by water depth (Bancroft, Gawlik, and Rutchey, 2002; Beerens and others, 2011). In agricultural fields, highest species richness was observed at depths of 10-15 cm and 35-40 cm (Elphick and Oring, 2003) and highest densities of foraging dabbling ducks occurred in large areas of shallow water (Tajiri and Ohkawara, 2013). Elsewhere, small shorebirds used water depths of $<6 \mathrm{~cm}$, medium shorebirds used water depths of $<12 \mathrm{~cm}$ (Twedt, 2013), and dabbling ducks used water depths of 22-25 cm (Isola and others, 2000).

Water availability and management, as well as underlying pond topography, influence water depth and habitat availability in Project ponds. Although increased topography only supported more roosting dabbling ducks and medium shorebirds at the pond scale, we determined that it was beneficial for most guilds at the grid scale. Some ponds created for salt production may have a relatively uniform pond bottom, with the largest changes in topography being related to borrow ditches adjacent to the levee and the shoreline that gradually transitions from the borrow ditch to the top of the levee. Increasing managed wetland topography to provide a range of foraging depths for different species has been recommended elsewhere (Weber and Haig, 1996; Taft and others, 2002), and our results suggest that increasing small-scale topography in shallow water Project ponds may improve habitat for a number of guilds and species.

In deeper ponds, Project managers have added islands with gradually sloping edges to modify topography and increase shallow foraging and roosting area availability for shorebirds and other guilds. Islands and their associated features were previously determined to influence the abundance of wintering waterbirds within Project ponds (Ackerman and others, 2014). Here we evaluated the importance of islands in comparison to other habitat variables and determined, based on variable importance rankings, that islands may be as critical as water depth, salinity, and other pond characteristics for foraging and roosting birds from multiple guilds. Other results from our study indirectly point to the importance of islands as well. For example, we observed a parabolic relation between shorebird abundance and water depth at the pond scale, where abundances were highest in very shallow ponds $(0-0.25 \mathrm{~m})$ and in the deepest ponds $(2.5 \mathrm{~m})$. This relation likely is owing to shorebird use of islands and their adjacent shallow waters in deeper ponds.

Artificial islands can provide important functions for foraging and roosting waterbirds, particularly in areas where natural habitats have been compromised (Burton, Evans, and Robinson, 1996). One advantage of island habitat is that it can afford protection from terrestrial predators and other types of disturbance (Giroux, 1981). In addition to increasing shallow foraging habitat, island creation also may increase deep water foraging areas for benthic-foraging diving ducks and fish-eating species within Project ponds because it often is accomplished by excavating adjacent pond sediments. Availability of island and levee edge habitat is essential for shorebirds not only for foraging, but also to meet their roosting needs. Maintenance of multiple roost site options is important for some species that use several sites, depending on factors such as distance to foraging area (Van Gils and others, 2006), windspeed, disturbance (Peters and Otis, 2007), daily tidal cycles, and seasonal habitat needs (Conklin, 
Colwell, and Fox-Fernandez, 2008). However, roost site fidelity can vary by region; Dunlin have high roost site fidelity in Tagus Estuary, Portugal, and low roost site fidelity in Humboldt Bay, California (Dias and others, 2006; Conklin and Colwell, 2008). Several habitat characteristics may be important for roosting shorebirds. For example, salt pans managed with shallow water can serve as important high-tide roosting sites for shorebirds (Catry and others, 2011).

Next to depth, distance to levee was the variable that significantly influenced the most foraging and roosting guilds at the grid scale. Abundances of all non-waterfowl guilds except for roosting terns were greater in grids closer to levees, indicating the broad importance of levee habitat for both roosting and foraging birds. Internal levees in particular may be cut off from the pond exterior and function as high-elevation, disturbance-free islands. Water control structures installed within levees provide a connection point between pond borrow ditches and SFB or adjacent sloughs. Thus, within the pond, deep areas near levees have high water circulation and may contain important prey resources such as fish that attract foraging piscivores to these sites. Shorebirds rely on levees as high-tide roosting sites, but also use shallow water near levee edges for foraging, as shown by radio-marked Western Sandpipers in the South Bay that selected salt pond levees at low and high tides (Warnock and Takekawa, 1995).

Many of the guilds and species we evaluated were most abundant in lower salinity (mixohaline, about $0.5-30 \mathrm{ppt}$ ) ponds, although small and medium shorebirds showed no relation to salinity, and foraging eared grebes and gulls were associated with high-salinity ponds. Some waterbird species may avoid high-salinity ponds because of their own physiological constraints (Gordus, Shivaprasad, and Swift, 2002) or limitations in prey resources (Sánchez, Green, and Castellanos, 2006). Common prey taxa of waterfowl and piscivores (including amphipods, polychaetes, and copepods, and several small fish species) often are most abundant in mixohaline environments (Thompson, Lowe, and Kellogg, 2000; Thompson and others, 2013). In addition to mixohaline invertebrates, many shorebird species also can take advantage of invertebrates such as Artemia and Ephydra spp., commonly present in highsalinity environments (Masero, 2003; Takekawa and others, 2009), which may explain why these guilds showed no relation to pond salinity. Eared grebes are hypersaline specialists that forage on Artemia and other high-salinity-associated prey during winter and migratory periods (Cullen, Jehl, and Neuchterlein, 1999). Our results suggest that, although it is clearly important to maintain high-salinity pond habitat to meet eared grebe foraging needs in the Project area, roosting birds may be able to take advantage of ponds across a much broader array of salinities.

Invertebrate prey is an important driver of waterbird abundance and species richness. In Portugal, medium shorebirds foraged in man-made salt pans during low tide rather than adjacent mudflats, suggesting the importance of these alternative habitats for providing shorebird prey resources (Dias and others, 2014). Invertebrate densities also can drive waterbird community composition within salt pans (Horvath and others, 2013). Prior research suggests that the invertebrate composition of Project ponds could support 79-102 percent of the energy needs of diving ducks, and 29-52 percent of the energy needs of small shorebirds (Brand and others, 2014); however, we lacked invertebrate data at the appropriate temporal and spatial scales to directly investigate relations between prey densities and waterbird abundance in this modeling effort. Ongoing (for example, Eden Landing Ecological Reserve ponds E12/13) and future studies of relations among salinity, depth, invertebrate communities, and waterbird density in experimental ponds may help identify important invertebrate taxa and management methods for maintaining them.

Pond area had a positive effect on abundance for diving ducks, piscivores, terns, and waders, which was similar to findings at a restoration site in Southern Spain that included man-made ponds (Sebastián-González and Green, 2013). Here, larger ponds supported higher bird abundances and species richness, which is consistent with the idea that, as wetland area increases, additional microhabitats become available and more species can use them (Sebastián-González and Green, 2013). 
Interestingly, we determined that the pond area did not have an influence on shorebird and dabbing duck abundances. One explanation might be that tidal marsh vegetation has only been established within three restored Project ponds thus far, leaving most of the Project landscape as interconnected, open water habitat that may function for some species as a larger area for foraging, roosting, and predator evasion. As vegetation begins to grow in additional restored ponds, remaining open water ponds will become increasingly isolated from each other and surrounded by vegetation, which could have effects on predator access and waterbird use (Bennett, Radford, and Haslem, 2006; Sebastián-González and Green, 2013). As Project restorations progress and mature, additional work on the effect of pond area and proximity to other suitable habitat in the mosaic of tidal wetlands and ponds may be critical to determine habitat requirements for species that are not sensitive to pond area under current conditions.

During our study period, about 13 percent of the surveyed Project area was restored to tidal flow under Phase I restorations. As ponds transition from open water habitat to fully vegetated tidal marsh, they first develop into tidal mudflat habitat that can provide foraging and roosting areas for shorebirds and other guilds. Passive tidal marsh restoration results in sediment accretion over time as tidewater brings substrate into the pond, and eventually allows the pond to reach elevations suitable for marsh plant colonization (Brand and others, 2012). Much like initial findings from work on waterbird use of breached habitat in the former salt ponds in North San Francisco Bay (Athearn, Takekawa and Shinn, 2009), our study suggests that ponds breached for wetland restoration may provide important foraging habitat for shorebirds during this transitional phase. Breached pond mudflat habitat typically is available at a different point in the tidal cycle than open Bay mudflat, and thus may increase the time period over which shorebirds have foraging opportunities each day. However, our results also indicated that diving duck and piscivore abundances were not associated with breached ponds, perhaps because water levels were not deep enough to support foraging and roosting by these guilds for extended periods each day.

We noted no influence of proximity to urban areas on bird abundance, and that the influence of pond proximity to the edge of SFB was limited to diving ducks and gulls. Adair and others (1996) also reported high abundances of diving ducks in coastal ponds that were close to estuary foraging sites. In the Tagus Estuary, Portugal, dunlin foraged within $5 \mathrm{~km}$ of their roosting site (Dias and others, 2006). Only four of our ponds were greater than $5 \mathrm{~km}$ from SFB, suggesting that most ponds likely were within a reasonable flight distance for small shorebirds to move to mudflats at low tide. Radio-marked Western sandpipers used mudflats immediately adjacent to the salt pond complexes in SFB and regularly moved between the two habitats (Warnock and Takekawa, 1996).

Human activity may alter habitat selection by sensitive waterbird species (Peters and Otis, 2007; Meager, Schlacher, and Nielsen, 2012); therefore, we evaluated the influence of public and hunting access on abundance. We noted positive relations between small shorebird and gull abundances and the percentage of pond levees open to the public. However, the proportion of levees open to hunting negatively influenced the abundance of piscivores, waders, and dabbling ducks. Brochet and others (2009) also reported a negative influence of hunting on dabbling ducks in the Camargue marshes of France. Murray and others (2013) reported that human access had a negative influence on diving ducks in urban ponds, although we did not note any effect on diving duck abundance. Our results differ somewhat from the findings of Trulio and Sokale (2008), who noted that trail use did not positively or negatively influence the shorebird abundance in the Project ponds. However, as with our findings on levees open to hunting, Trulio and others (2012) reported that waterfowl responded to new trail use in the Project area by swimming or flying away and that dabbling duck abundance was lower near levees with trails compared to those without trails. Our results on gulls were similar to those noted in another urbanized estuary in Portugal, where gull abundance increased with increasing human presence (Rosa, Palmeirim, and Moreira, 2003). 
Our analyses did not comprehensively test the effects of hunting on abundance because we did not have reliable information on hunting blind placement in all Project ponds for the entire length of the study and, therefore, only considered effects from levee-based hunting. Likewise, for other types of public access, we only evaluated waterbird response to levees that were open to the public or not, but we did not have appropriate data to distinguish among levels of actual use of trails adjacent to ponds. Although some experimental work has been conducted in the Project areas to evaluate these parameters (Trulio and Sokale, 2008; Trulio and others, 2012), additional studies may be warranted given the variation in responses of species to different types and levels of disturbance, and the potential for local population consequences to some species (Gill, Norris, and Sutherland, 2001).

\section{Management Implications}

Given that much of the habitat conversion planned for Project ponds has not yet occurred and that current management is geared towards optimizing conditions for waterbirds, present bird numbers may be approaching peak values for this former salt pond system. With the planned conversion of 5090 percent of Project ponds to tidal marsh, optimizing the quality of remaining open water habitat to maintain waterbird abundance and diversity within a smaller footprint may be needed. We identified several significant landscape and micro-habitat level characteristics for key guilds and species of waterbirds, and our results support evidence from other studies suggesting that a mosaic of habitat features is important for avian diversity and abundance (Burger, Niles, and Clark, 1997; Bennett, Radford, and Haslem, 2006; Mohd-Azlan, Noske, and Lawes, 2015). Many of the habitat characteristics that we identified as important can be manipulated by managers to benefit one or more taxa. In many cases, the ranges of optimal habitat values overlap among guilds and species, providing the opportunity to manage individual or groups of ponds to benefit several species. For example:

- Depth was a significant driver of abundance; however, guilds had variable relations to depth, with abundances peaking across a wide range $(0-2.5 \mathrm{~m})$ at the pond and grid scales. In addition to water level manipulation, increasing topography and islands within ponds may be one approach to meeting the depth needs of multiple species.

- Our grid-scale results suggest that increasing small-scale topography from 0.12 to $1.27 \mathrm{~m}$ in shallow water Project ponds may increase foraging use by most of the guilds and species we studied. Experimental studies to evaluate the response of waterbirds to various topographic manipulations may further inform management of this habitat feature.

- Presence of islands was an important pond feature that influenced the abundance of several guilds as much as depth and salinity. Our analyses suggest that maintaining islands may help sustain foraging and roosting waterbirds from multiple guilds.

- Close proximity to levees was important for roosting and foraging birds from all non-waterfowl guilds, suggesting that maintaining levees and the habitat heterogeneity immediately adjacent to them may increase abundances of several guilds. Additional research to determine which levee features attract each guild may be informative.

- Maintaining water salinity of $\leq 33$ ppt may benefit foraging and roosting dabbling and diving ducks (including northern shoveler and ruddy duck), piscivores, terns, and waders. However, gulls and eared grebes may require ponds maintained at much higher salinities ( $\geq 109 \mathrm{ppt})$ for foraging. Foraging and roosting small and medium shorebirds may be able to take advantage of conditions across multiple salinities. 
- Invertebrate prey availability is known to be a main driver of foraging waterbird abundance, yet we lacked information on invertebrate density to inform our models. Ongoing and future studies of relations among salinity, depth, invertebrate communities, and waterbird density in experimental ponds may help identify important invertebrate taxa and management methods for maintaining them.

- Maintaining ponds with large areas of open water $\left(>1.0 \mathrm{~km}^{2}\right)$ may benefit foraging and roosting diving ducks, piscivores, terns, gulls, waders, and eared grebes. As Project restorations progress and mature, additional work on the effects of pond area and proximity to other suitable habitat in the mosaic of tidal wetlands and ponds may help determine area requirements for all guilds and species.

- Foraging small shorebirds were the only guild for which abundance was positively related to breached ponds. As Project restorations progress, future work to investigate temporal and spatial use of breached habitat by all guilds may inform management of post-breach transitional habitat to maximize waterbird abundances.

- We noted variable responses to hunting and other public access among guilds. The information available for these variables limited our analyses; thus, additional studies may be warranted given the variation in responses of species to different types and levels of disturbance, and the potential for local population consequences to some species.

- Eared grebes are hypersaline specialists with unique depth and salinity requirements that often do not overlap those of other guilds and species. Additional targeted studies on movements and seasonal habitat use of eared grebes may inform management opportunities for this species.

- Our model results can be used in future analyses to evaluate the effects of different management scenarios on waterbird abundances, including weighing costs and benefits of maintaining individual or groups of ponds or converting them to tidal marsh.

This modeling exercise used a detailed 13-year dataset to identify trends and key drivers of waterbird abundance, underscoring the value of restoration monitoring and research to inform adaptive management. The results presented here are powerful tools for advancing adaptive management actions that can help reach the long-term goals of this 50-year Project.

\section{Acknowledgments}

We thank the Don Edwards San Francisco Bay National Wildlife Refuge Complex (Anne Morkill, Eric Mruz, Joy Albertson, Rachel Tertes), Eden Landing Ecological Reserve, South Bay Salt Pond Restoration Project (Laura Valoppi and John Bourgeois), and U.S. Fish and Wildlife Service Inventory and Monitoring Program (Giselle Block, Kaylene Keller) for logistical and project support. J. Falgout and B. Williams at U.S. Geological Survey (USGS) Core Science Analytics, Synthesis, and Libraries in Denver provided integral assistance in using the Simple Linux Utility for Resource Management (SLURM). We thank N. Athearn and L. Brand (USGS), and J. Bluso-Demers, C. Burns, and N. Washburn (San Francisco Bay Bird Observatory [SFBBO]) for waterbird project coordination; J. Shinn (USGS) and J. Scullen (SFBBO) for data management; K. Spragens (USGS) for helpful discussion; J. Yee (USGS) for statistical advice; and T. Graham (USGS) for review. We thank the many field technicians that conducted surveys and entered data throughout the years of this project. 


\section{References Cited}

Ackerman, J.T., Hartman, C.A., Herzog, M.P., Smith, L.M., Moskal, S.M., De La Cruz, S.E.W., Yee, J.L., and Takekawa, J.Y., 2014, The critical role of islands for waterbird breeding and foraging habitat in managed ponds of the South Bay Salt Pond Restoration Project, South San Francisco Bay, California: U.S. Geological Survey Open-File Report 2014-1263, 108 p.

Adair, S.E., Moore, J.L., and Kielm, Jr., W.H., 1996, Diving duck use of coastal ponds - An analysis of alternative hypotheses: The Journal of Wildlife Management, v. 60, p. 83-93.

Athearn, N.D., Takekawa, J.Y., Bluso-Demers, J.D., Shinn, J.M., Brand, L.A., Robinson-Nilsen, C.W., and Strong, C.M., 2012, Variability in habitat value of commercial salt production pondsImplications for waterbird management and tidal marsh restoration planning: Hydrobiologia, v. 697, p. $139-155$.

Athearn, N.D., Takekawa, J.Y., Jaffe, B., Hattenbach, B.J., and Foxgrover, A.C., 2010, Mapping elevations of tidal wetland restoration sites in San Francisco Bay-Comparing accuracy of aerial lidar with a singlebeam echosounder: Journal of Coastal Research, v. 26, p. 312-319.

Athearn, N.D., Takekawa, J.Y., and Shinn, J.M., 2009, Avian response to early tidal salt marsh restoration at former commercial salt evaporation ponds in San Francisco Bay, California, USA: Natural Resources and Environmental Issues, v. 15, p. 77-86.

Bancroft, G.T., Gawlik, D.E., and Rutchey, K., 2002, Water depths in the northern Everglades of Florida, USA: Journal of the Waterbird Society, v. 25, p. 265-277.

Beerens, J.M., Gawlik, D.E., Herring, G., and Cook, M.I., 2011, Dynamic habitat selection by two wading bird species with divergent foraging strategies in a seasonally fluctuating wetland: The Auk, v. 128 , p. $651-662$.

Bennett, A.F., Radford, J.Q., and Haslem, A., 2006, Properties of land mosaics - Implications for nature conservation in agricultural environments: Biological Conservation, v. 133, p. 250-264.

Brand, L.A., Smith, L.M., Takekawa, J.Y., and others, 2012, Trajectory of early tidal marsh restoration-Elevation, sedimentation and colonization of breached salt ponds in the northern San Francisco Bay: Ecological Engineering, v. 42, p. 19-29.

Brand, L.A., Takekawa, J.Y., Shinn, J., Graham, T., Buffington, K., Gustafson, K. Ben, Smith, L.M., and Spring, S.E., 2014, Effects of wetland management on carrying capacity of diving ducks and shorebirds in a coastal estuary: Waterbirds, v. 37, p. 52-67.

Brochet, A.L., Gauthier-Clerc, M., Mathevet, R., Béchet, A., Mondain-Monval, J.Y., and Tamisier, A., 2009, Marsh management, reserve creation, hunting periods and carrying capacity for wintering ducks and coots: Biodiversity and Conservation, v. 18, p. 1,879-1,894.

Burger, J., Niles, L., and Clark, K.E., 1997, Importance of beach, mudflat and marsh habitats to migrant shorebirds on Delaware Bay: Biological Conservation, v. 79, p. 283-292.

Burnham, K.P., and Anderson, D.R., 2002, Model selection and multimodel inference-A practical information-theoretic approach (2d ed.): New York, Springer-Verlag, 488 p.

Burton, N.H.K., Evans, P.R., and Robinson, M.A., 1996, Effects of shorebird numbers of disturbance, the loss of a roost site and its replacement by an artificial island at Hartlepool, Cleveland: Biological Conservation, v. 77, p. 193-201.

Catry, T., Alves, J.A., Andrade, J., Costa, H., Dias, M.P., Fernandes, P., Leal, A., Lourenço, P.M., Martins, R.C., Moniz, F., Pardal, S., Rocha, A., Santos, C.D., Encarnação, V., and Granadeiro, J.P., 2011, Long-term declines of wader populations at the Tagus Estuary, Portugal-A response to global or local factors?: Bird Conservation International, v. 21, p. 438-453.

Conklin, J.R., and Colwell, M.A., 2008, Individual associations in a wintering shorebird populationDo dunlin have friends?: Journal of Field Ornithology, v. 79, p. 32-40. 
Conklin, J.R., Colwell, M.A., and Fox-Fernandez, N.W., 2008, High variation in roost use by Dunlin wintering in California-Implications for habitat limitation: Bird Conservation International, v. 18, p. 275-291.

Cullen, S.A., Jehl, J.R., and Neuchterlein, G.L., 1999, Eared grebe (Podiceps nigricollis): Cornell Lab of Ornithology Birds of North America website, accessed January 2016, at http://bna.birds.cornell.edu/bna/species/433.

Dias, M.P., Granadeiro, J.P., Lecoq, M., Santos, C.D., and Palmeirim, J.M., 2006, Distance to high-tide roosts constrains the use of foraging areas by dunlins-Implications for the management of estuarine wetlands: Biological Conservation, v. 131, p. 446-452.

Dias, M.P., Lecoq, M., Moniz, F., and Rabaça, J.E., 2014, Can human-made saltpans represent an alternative habitat for shorebirds?-Implications for a predictable loss of estuarine sediment flats: Environmental Management, v. 53, p. 163-171.

EDAW and others, 2007, Final environmental impact statement/environmental impact report for the South Bay Salt Pond Restoration Project: Prepared for U.S. Fish and Wildlife Service and California Department of Fish and Game, p. 1750.Elphick, C.S., and Oring, L.W., 2003, Conservation implications of flooding rice fields on winter waterbird communities: Agriculture, Ecosystems and Environment, v. 94, p. 17-29.

Fournier, D.A., Skaug, H.J., Ancheta, J., Ianelli, J., Magnusson, A., Maunder, M.N., Nielsen, A., and Sibert, J., 2012, AD Model Builder-Using automatic differentiation for statistical inference of highly parameterized complex nonlinear models: Optimization Methods and Software, v. 27, p. 233-249.

Gill, J.A., Norris, K., and Sutherland, W.J., 2001, Why behavioral responses may not reflect the population consequences of human disturbance: Biological Conservation, v. 97, p. 265-268.

Giroux, J-F., 1981, Use of artificial islands by nesting waterfowl in southeastern Alberta: Journal of Wildlife Management, v. 45, p. 69-679

Goals Project, 1999, Baylands ecosystem habitat goals-A report of habitat recommendations: Prepared by the San Francisco Bay Area Wetlands Ecosystem Goals Project, U.S. Environmental Protection Agency, San Francisco, California/San Francisco Bay Regional Water Quality Control Board, Oakland, California, 209 p. plus appendix.

Gordus, A.G., Shivaprasad, H.L., and Swift, P.K., 2002, Salt toxicosis in ruddy ducks that winter on an agricultural evaporation basin in California: Journal of Wildlife Diseases, v. 38, p. 124-131.

Hastie, T.J., and Tibshirani, R.J., 1990, Generalized additive models: Boca Raton, Florida, CRC Press, $352 \mathrm{p}$.

Horvath, Z., Vad, C.F., Voros, L., and Boros, E., 2013, The keystone role of anostracans and copepods in European soda pans during the spring migration of waterbirds: Freshwater Biology, v. 58, p. 430440.

Isola, C.R., Colwell, M.A., Taft, O.W., and Safran, R.J., 2000, Interspecific differences in habitat use of shorebirds and waterfowl foraging in managed wetlands of California's San Joaquin Valley:

Waterbirds, v. 23, p. 196-203.

Lewis, E.L., 1980, The Practical Salinity Scale 1978 and its antecedents: Journal of Oceanic Engineering, v. 5, p. 3-8.

Masero, J.A., 2003, Assessing alternative anthropogenic habitats for conserving waterbirds_-Salinas as buffer areas against the impact of natural habitat loss for shorebirds: Biodiversity and Conservation, $\mathrm{v}$. 12 , p. $1,157-1,173$.

Meager, J.J., Schlacher, T.A., and Nielsen, T., 2012, Humans alter habitat selection of birds on oceanexposed sandy beaches: Diversity and Distributions, v. 18, p. 294-306.

Mohd-Azlan, J., Noske, R.A., and Lawes, M.J., 2015, The role of habitat heterogeneity in structuring mangrove bird assemblages: Diversity, v. 7, p. 118-136. 
Morrison, R.I.G., 2001, Trends in shorebird populations in North America using Breeding Bird Survey data: Bird Trends, v. 8, p. 12-15.

Murray, C.G., Kasel, S., Loyn, R.H., Hepworth, G., and Hamilton, A.J., 2013, Waterbird use of artificial wetlands in an Australian urban landscape: Hydrobiologia, v. 716, p. 131-146.

Page, G.W., Stenzel, L.E., and Kjelmyr, J.E., 1999, Overview of shorebird abundance and distribution in wetlands of the Pacific Coast of the contiguous United States: The Condor, v. 101, p. 461-471.

Peters, K.A., and Otis, D.L., 2007, Shorebirds roost-site selection at two temporal scales-Is human disturbance a factor?: Journal of Applied Ecology, v. 44, p. 196-209.

R Core Team, 2014: R-A language and environment for statistical computing: Vienna Austria, $\mathrm{R}$ Foundation for Statistical Computing, accessed August 2015, at http://www.R-project.org/.

Richmond, O.M.W., Dulava, S., Strong, C.M., and Albertson, J.D., 2014, San Francisco Estuary midwinter waterfowl survey-2012 survey results and trend analysis (1981-2012): Prepared by the U.S. Fish and Wildlife Service Pacific Southwest Region National Wildlife Refuge System Inventory and Monitoring Initiative, Fremont, California, $54 \mathrm{p}$.

Rosa, S., Palmeirim, J., and Moreira, F., 2003, Factors affecting waterbird abundance and species richness in an increasingly urbanized area of the Tagus Estuary in Portugal: Waterbirds, v. 26, p. 226232.

Sánchez, M.I., Green, A.J., and Castellanos, E.M., 2006, Spatial and temporal fluctuations in presence and use of chironomid prey by shorebirds in the Odiel saltpans, south-west Spain: Hydrobiologia, v. 567, p. 329-340.

San Francisco Estuary Partnership, 2015, The state of the estuary 2015-Status and trends updates on 33 indicators of ecosystem health: San Francisco Estuary Partnership, Oakland, California, 92 p.

Sebastián-González, E., and Green, A.J., 2013, Habitat use by waterbirds in relation to pond size, water depth, and isolation-Lessons from a restoration in southern Spain: Restoration Ecology, v. 22, no. 3, p. 311-318.

Siegel, S.W., and Bachand, P.A.M., 2002, Feasibility analysis of South Bay Salt Pond Restoration, San Francisco Estuary, California: San Rafael, California, Wetlands and Water Resources, 228 p.

Skaug, H., Fournier, D., Nielsen, A., Magnusson, A., and Bolker, B., 2012, Generalized linear mixed models using AD Model Builder — R package version 0.7.2.12. http://glmmadmb.r-forge.rproject.org.

Stallings, L., 2004, South Bay salt ponds initial stewardship plan-Final environmental impact report/environmental impact statement: Prepared by Life Science!, Inc., Woodland, California, for U.S. Fish and Wildlife Service and California Department of Fish and Game.

Steere, J.T., and Schaefer, N., 2001, Restoring the estuary-Implementation strategy of the San Francisco Bay Joint Venture: Oakland, California, San Francisco Bay Joint Venture, 111 p.

Stenzel, L.E., and Page, G.W., 1988, Results of the first comprehensive shorebird census of San Francisco and San Pablo Bays: Wader Study Group Bulletin, v. 54, p. 43-48.

Taft, O.W., Colwell, M.A., Isola, C.R., and Safran, R.J., 2002, Waterbird responses to experimental drawdown-Implications for the multispecies management of wetland mosaics: Journal of Applied Ecology, v. 39, p. 987-1,001.

Tajiri, H., and Ohkawara, K., 2013, The effects of flooding and plowing on foraging site selection by wintering dabbling ducks in rice fields: Ornithological Science, v. 12, p. 127-136.

Takekawa, J.Y., Miles, A.K., Tsao-Melcer, D.C., Schoellhamer, D.H., Fregien, S., and Athearn, N.D., 2009, Dietary flexibility in three representative waterbirds across salinity and depth gradients in salt ponds of San Francisco Bay: Hydrobiologia, v. 626, p. 155-168. 
Tavares, D.C., Guadagnin, D.L., de Moura, J.F., Siciliano, S., and Merico, A., 2015, Environmental and anthropogenic factors structuring waterbird habitats of tropical coastal lagoons-Implications for management: Biological Conservation, v. 186, p. 12-21.

Thompson, B., Lowe, S., and Kellogg, M., 2000, Results of the benthic pilot study 1994-1997_Part 1-Macrobenthic assemblages of the San Francisco Bay-Delta, and their responses to abiotic factors: San Francisco, Califoirnia, San Francisco Estuary Institute, Technical Report 39, 40 p.

Thompson, B., Ranasinghe, J.A., Lowe, S., Melwani, A., and Weisberg, S.B., 2013, Benthic macrofaunal assemblages of the San Francisco Estuary and Delta, USA: Environmental Monitoring and Assessment, v. 185, p. 2,281-2,295.

Trost, R.E., and Sanders, T.A., 2009, Pacific Flyway Data Book-waterfowl harvest and status, hunter participation and success in the Pacific Flyway and United States. United States Fish and Wildlife Service. Portland, Oregon.

Trulio, L.A., and Sokale, J., 2008, Foraging shorebird response to trail use around San Francisco Bay: Journal of Wildlife Management, v. 72, p. 1,775-1,780.

Trulio, L., White, H., Sokale, J., and Tokatlian, K., 2012, Report on waterfowl response to trail use in the South Bay Salt Pond Restoration Project: San Jose, California, San Jose State University and others, $16 \mathrm{p}$.

Twedt, D.J., 2013, Foraging habitat for shorebirds in southeastern Missouri and its predicted future availability: Wetlands, v. 33, p. 667-678.

U.S. Fish and Wildlife Service, 2006, Waterfowl population status, 2006: U.S. Fish and Wildlife Service, $60 \mathrm{p}$.

Van Gils, J.A., Spaans, B., Dekinga, A., and Piersma, T., 2006, Foraging in a tidally structured environment by red knots (Calidris canutus) - Ideal, but not free: Ecology, v. 87, p. 1,189-1,202.

Warnock, N., Page, G.W., Ruhlen, T.D., Nur, N., Takekawa, J.Y., and Hanson, J.T., 2002, Management and conservation of San Francisco Bay salt ponds-Effects of pond salinity, area, tide, and season on Pacific Flyway waterbirds: Waterbirds, v. 25, p. 79-92.

Warnock, S.E., and Takekawa, J.Y., 1995, Habitat preferences of wintering shorebirds in a temporally changing environment-Western sandpipers in the San Francisco Bay Estuary: The Auk, v. 112, p. 920-930.

Warnock, S.E., and Takekawa, J.Y., 1996, Wintering site fidelity and movement patterns of western sandpipers Calidris mauri in the San Francisco Bay Estuary: Ibis, v. 138, p. 160-167.

Warton, D.I., 2005, Many zeros does not mean zero inflation-Cmparing the goodness-of-fit of parametric models to multivariate abundance data: Environmetrics, v. 16, p. 275-289.

Weber, L.M., and Haig, S.M., 1996, Shorebird use of South Carolina managed and natural coastal wetlands: The Journal of Wildlife Management, v. 60, p. 73-82.

Wood, J., Page, G., Reiter, M., Liu, L., and Robinson-Nilsen, C., 2010, Abundance and distribution of wintering shorebirds in San Francisco Bay, 1990-2008-Population change and informing future monitoring: Prepared by PRBO Conservation Science, Petaluma, California, and San Francsco Bay Bird Observatory, Milpitas, California, for Resources Legacy Fund, Sacramento, California, 56 p.

Zuur, A.F., Leno, E.N., Walker, N.J., Saveliev, A.A., and Smith, G.M., 2009, Mixed effects models and extensions in ecology with R: New York, Springer, 574 p. 


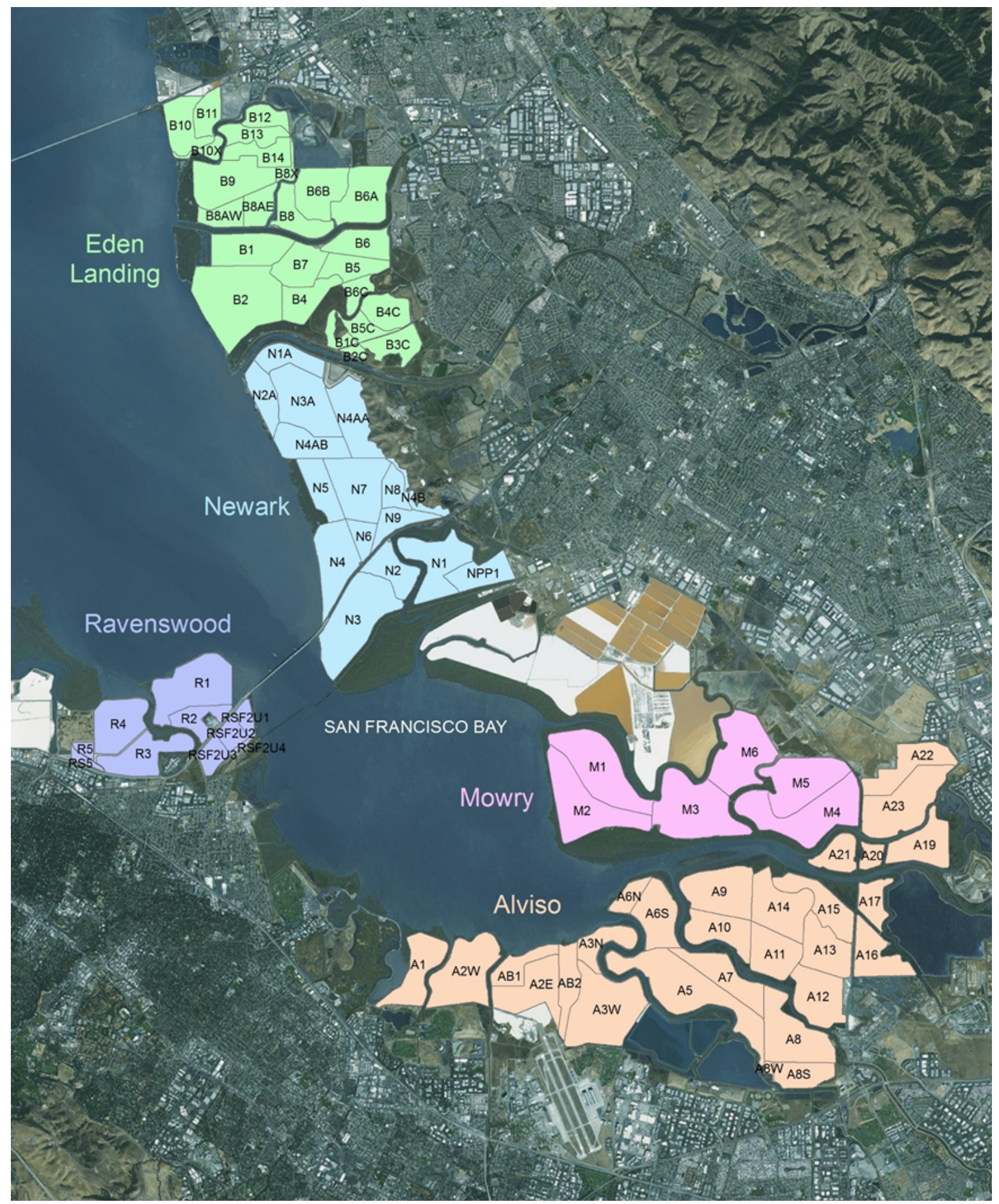

Figure 1. Former and current salt ponds grouped in several complexes in South San Francisco Bay, California. Ponds in Alviso and Ravenswood (both managed by Don Edwards San Francisco Bay National Wildlife Refuge), and the Eden Landing Ecological Reserve (California Department of Fish and Wildlife) are part of the South San Francisco Bay Restoration Project. Cargill, Inc. manages the Mowry and Newark pond complexes for salt production 
A

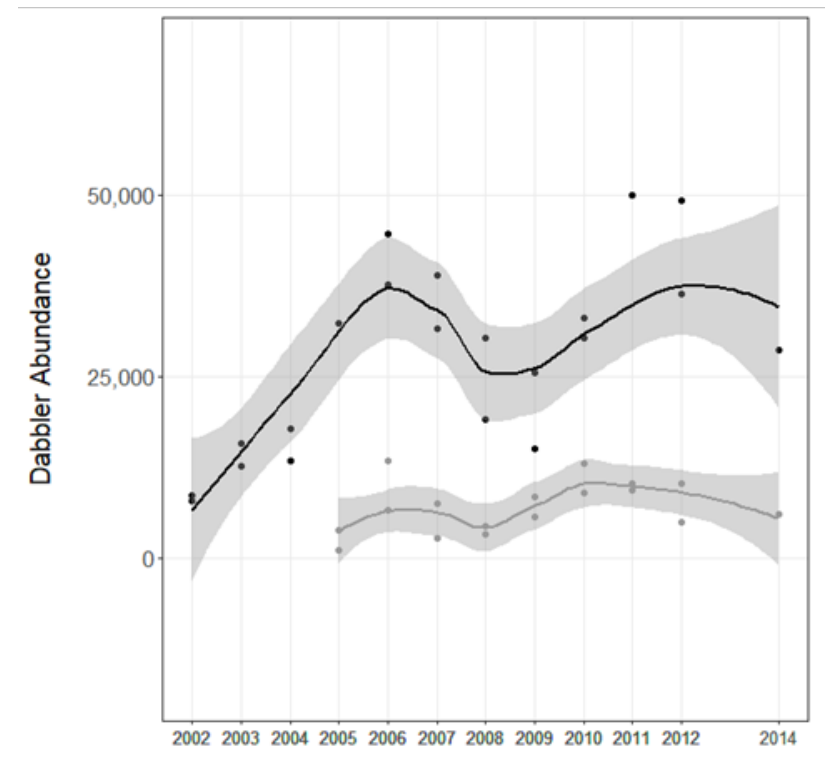

Year
B

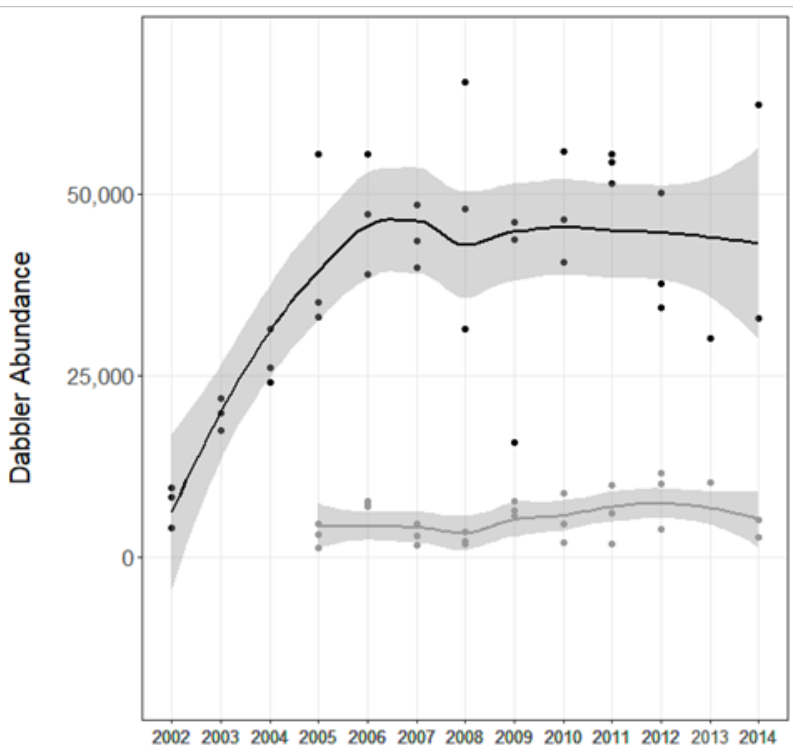

Year
C

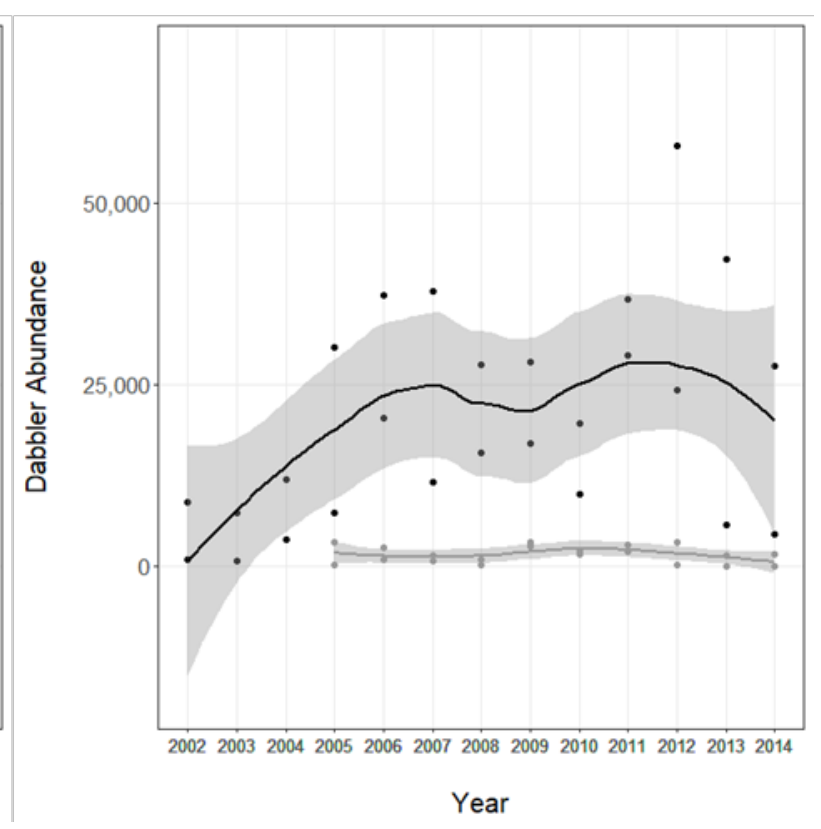

Figure 2. Dabbling duck population trends during fall (A), winter (B), and spring (C) in South Bay Salt Pond Restoration Project ponds (dark gray) and active salt production ponds (light gray), South San Francisco Bay, California. Points represent abundance of each monthly count, where fall includes October and November; winter includes December, January, and February; and spring includes March and April. Trend lines were created using locally weighted scatterplot smoothing in R. Counts were not conducted during fall 2013. Year corresponds to the year of fall, winter 2013 includes January and February 2014, and spring 2013 includes March and April 2014. 
A

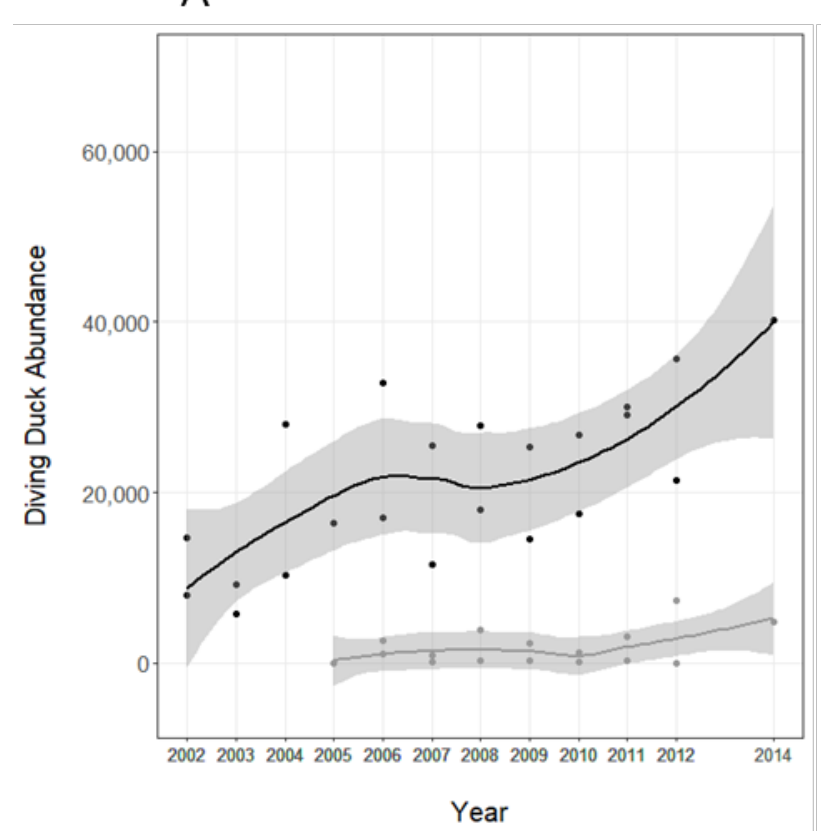

B

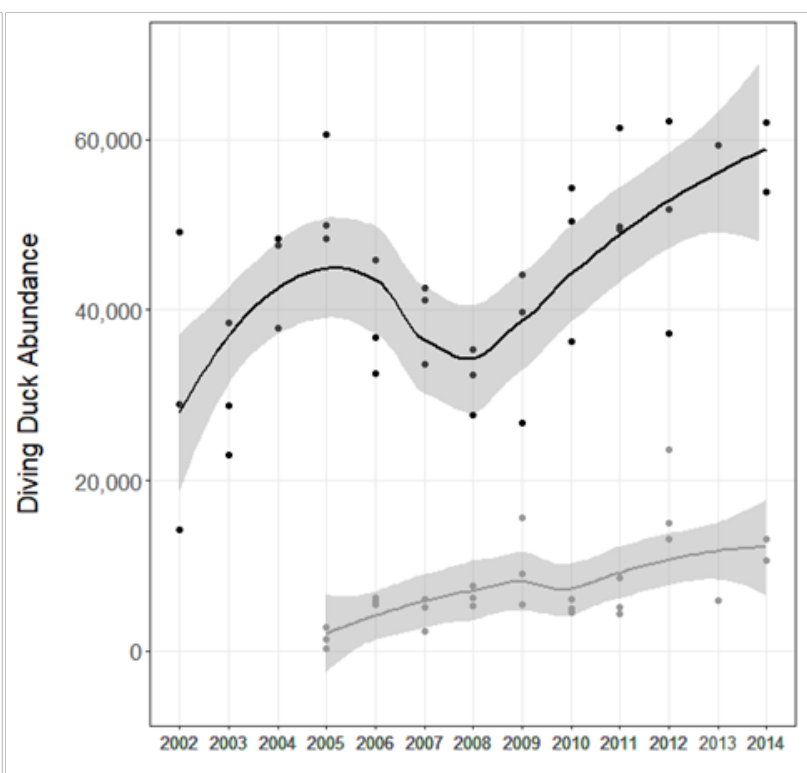

Year
C

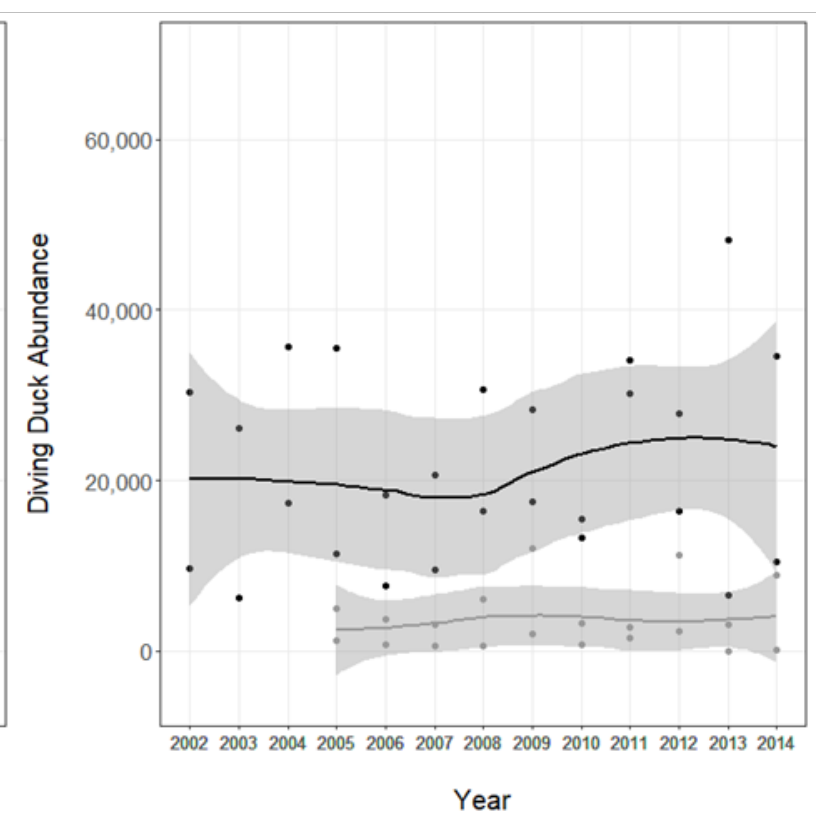

Figure 3. Diving duck population trend during fall (A), winter (B), and spring (C) in South Bay Salt Pond Restoration Project ponds (dark gray) and active salt production ponds (light gray), South San Francisco Bay, California. Points represent abundance of each monthly count, where fall includes October and November; winter includes December, January, and February; and spring includes March and April. Trend lines were created using locally weighted scatterplot smoothing in R. Counts were not conducted during fall 2013. Year corresponds to the year of fall, winter 2013 includes January and February 2014, and spring 2013 includes March and April 2014. 
A

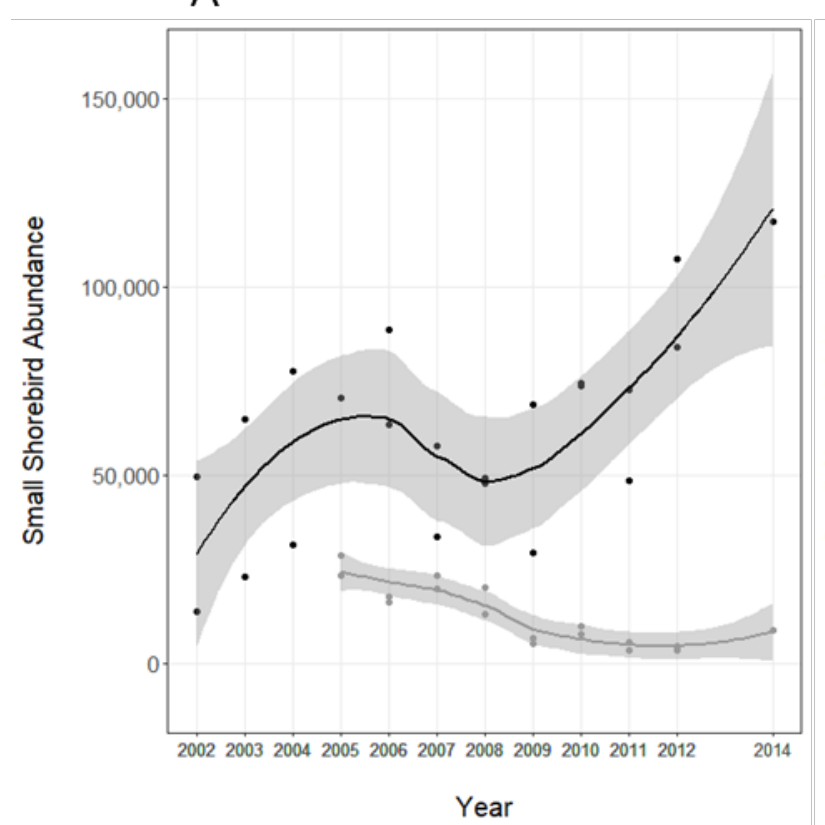

B

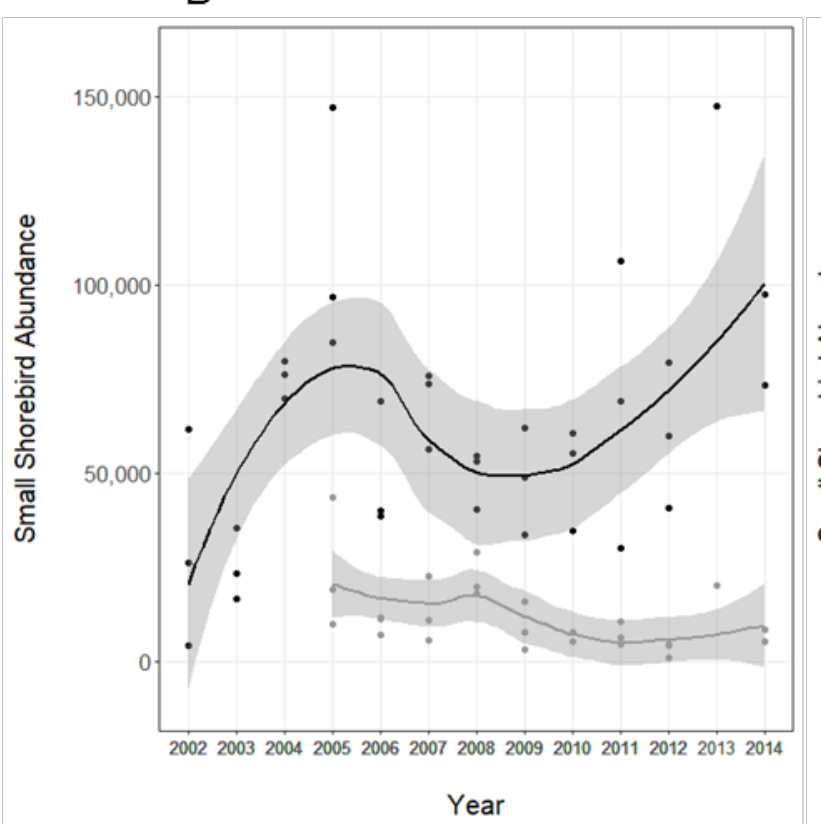

C

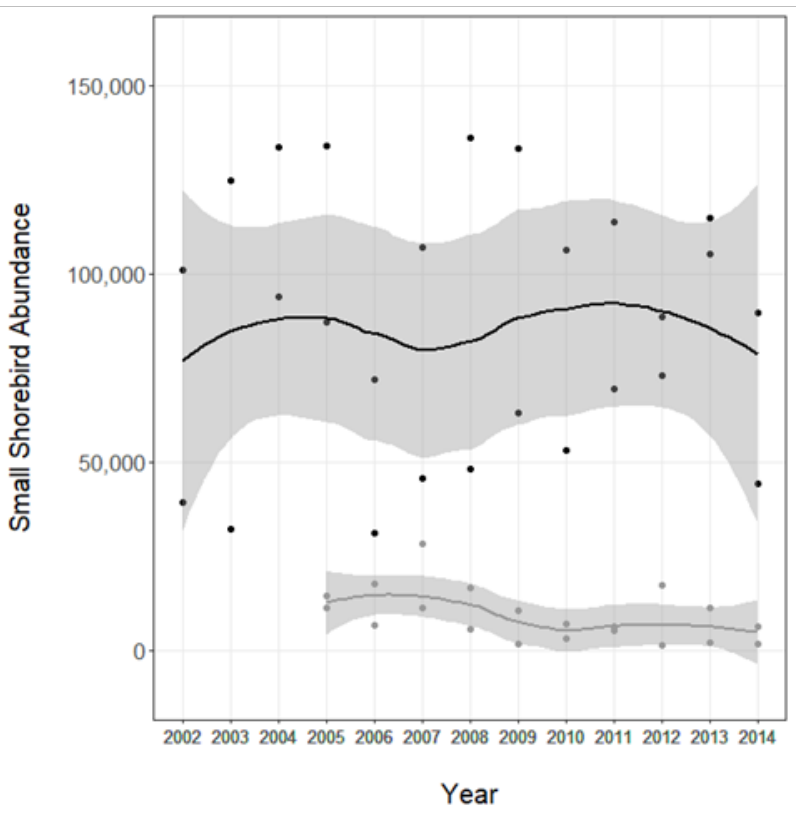

Figure 4. Small shorebird population trend during fall (A), winter (B), and spring (C) in South Bay Salt Pond Restoration Project ponds (dark gray) and active salt production ponds (light gray), South San Francisco Bay, California. Points represent abundance of each monthly count, where fall includes October and November; winter includes December, January, and February; and spring includes March and April. Trend lines were created using locally weighted scatterplot smoothing in R. Counts were not conducted during fall 2013. Year corresponds to the year of fall, winter 2013 includes January and February 2014, and spring 2013 includes March and April 2014. 
A

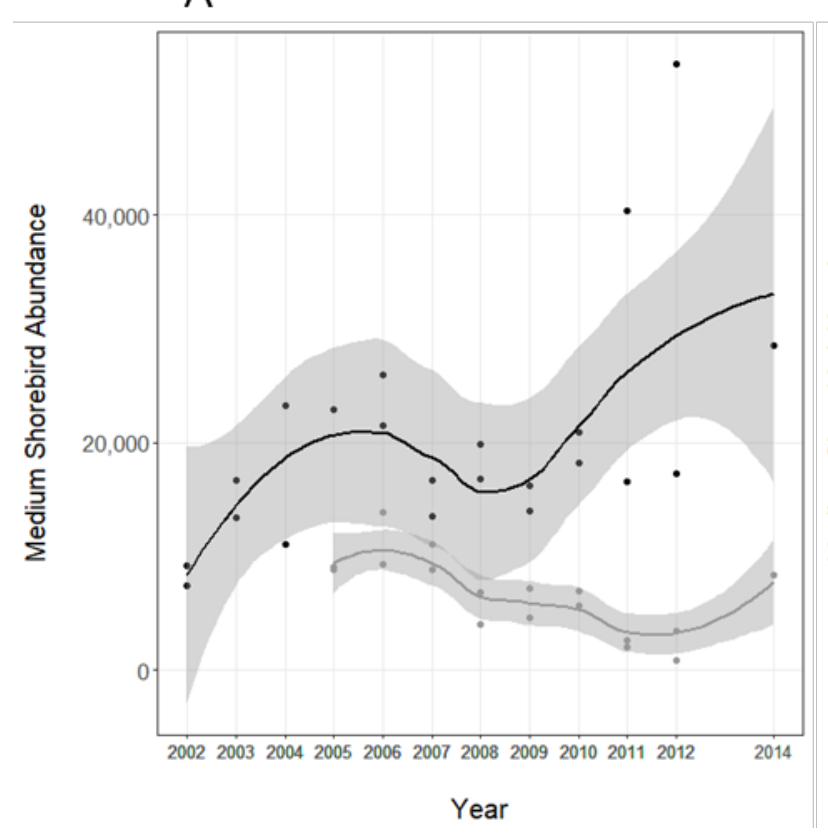

B

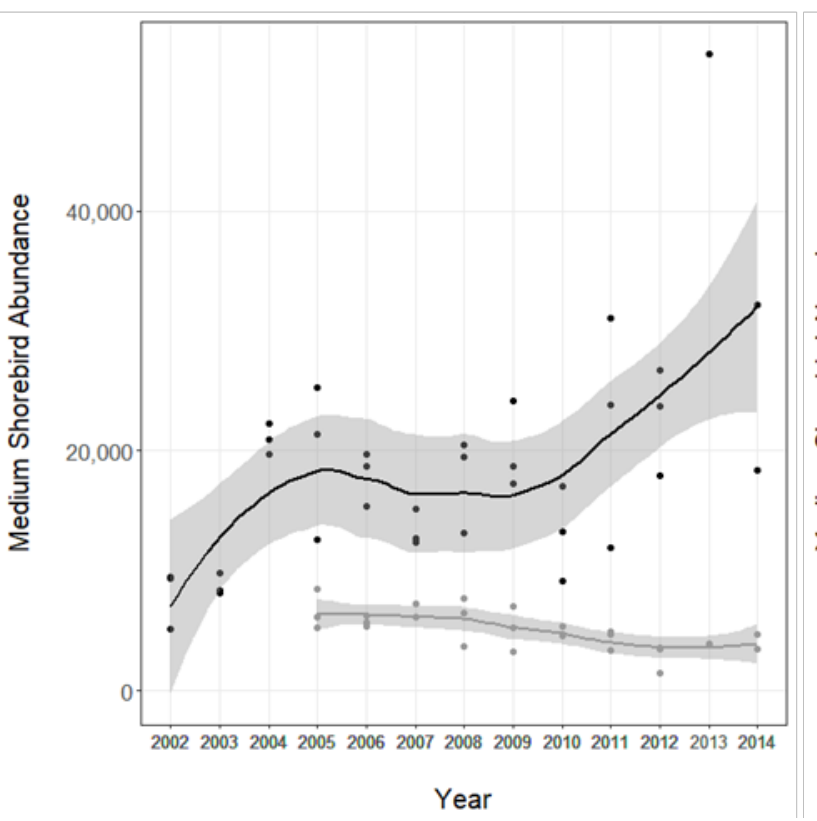

C

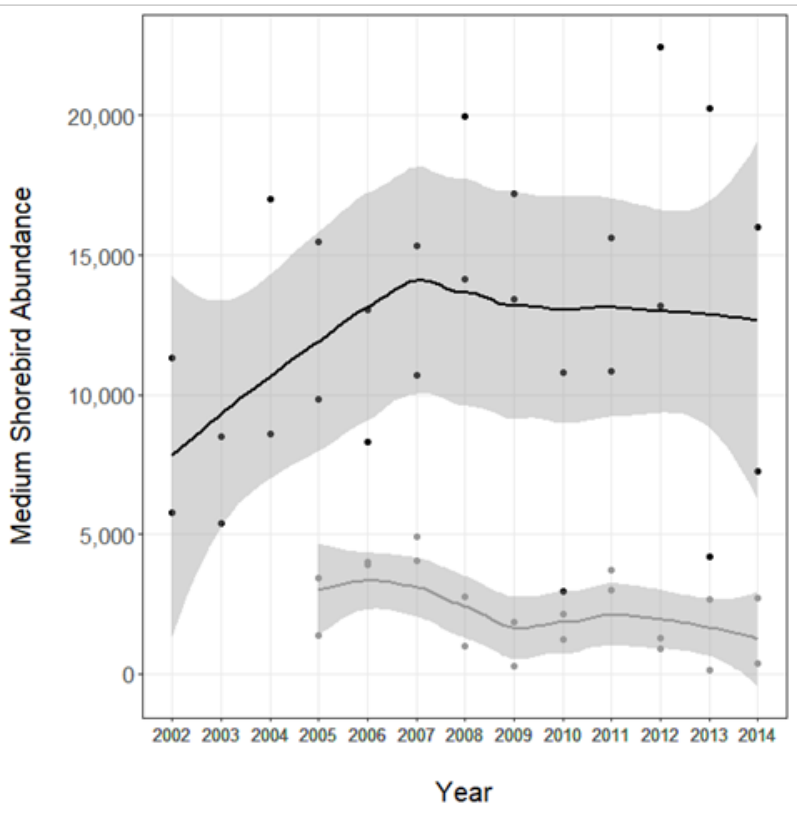

Figure 5. Medium shorebird population trend during fall (A), winter (B), and spring (C) in South Bay Salt Pond Restoration Project ponds (dark gray) and active salt production ponds (light gray), South San Francisco Bay, California. Points represent abundance of each monthly count, where fall includes October and November; winter includes December, January, and February; and spring includes March and April. Trend lines were created using locally weighted scatterplot smoothing in R. Counts were not conducted during fall 2013. Year corresponds to the year of fall, winter 2013 includes January and February 2014, and spring 2013 includes March and April 2014. 
A

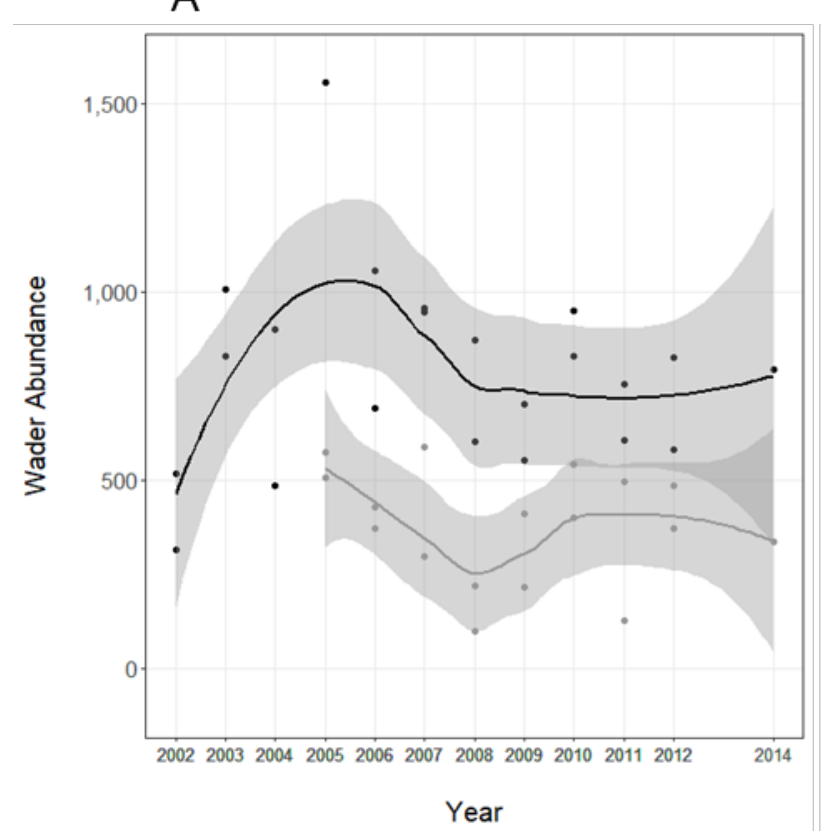

B

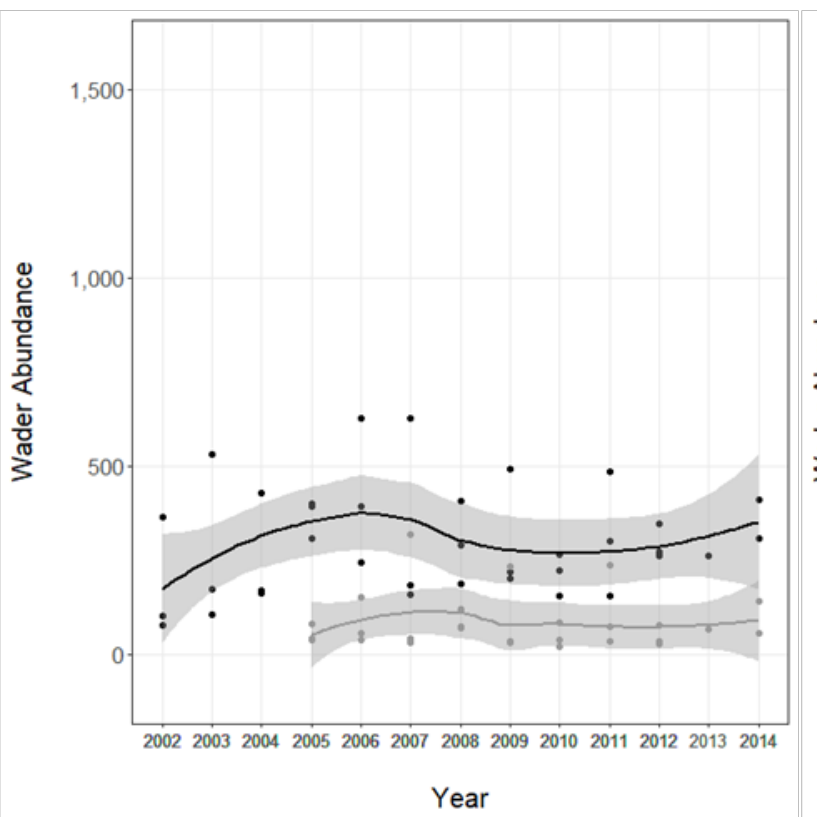

C

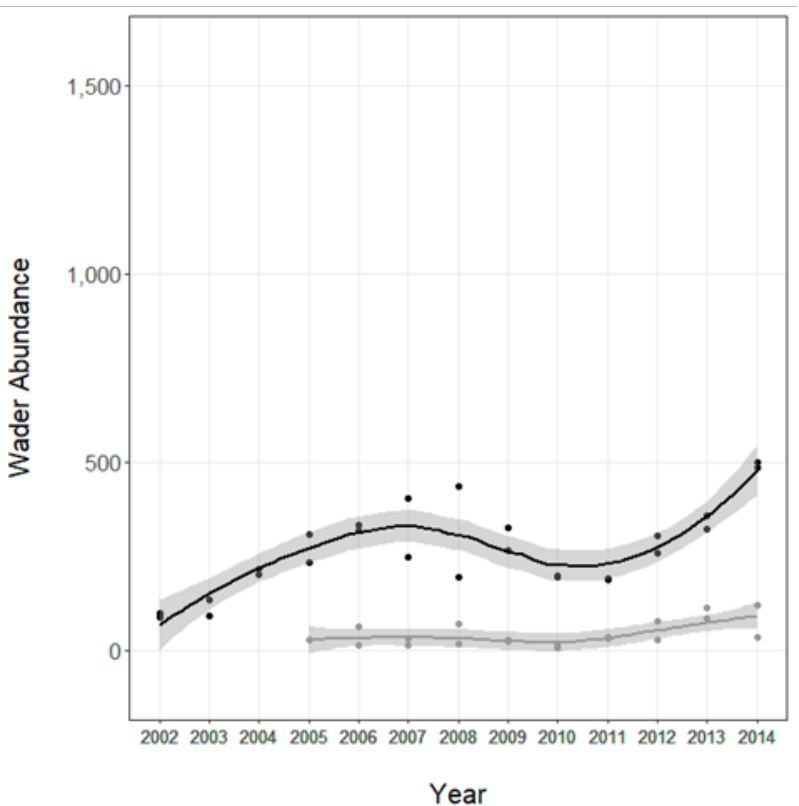

Figure 6. Wader population trend during fall (A), winter (B), and spring (C) in South Bay Salt Pond Restoration Project ponds (dark gray) and active salt production ponds (light gray), South San Francisco Bay, California. Points represent abundance of each monthly count, where fall includes October and November; winter includes December, January, and February; and spring includes March and April. Trend lines were created using locally weighted scatterplot smoothing in R. Counts were not conducted during fall 2013. Year corresponds to the year of fall, winter 2013 includes January and February 2014, and spring 2013 includes March and April 2014. 
A

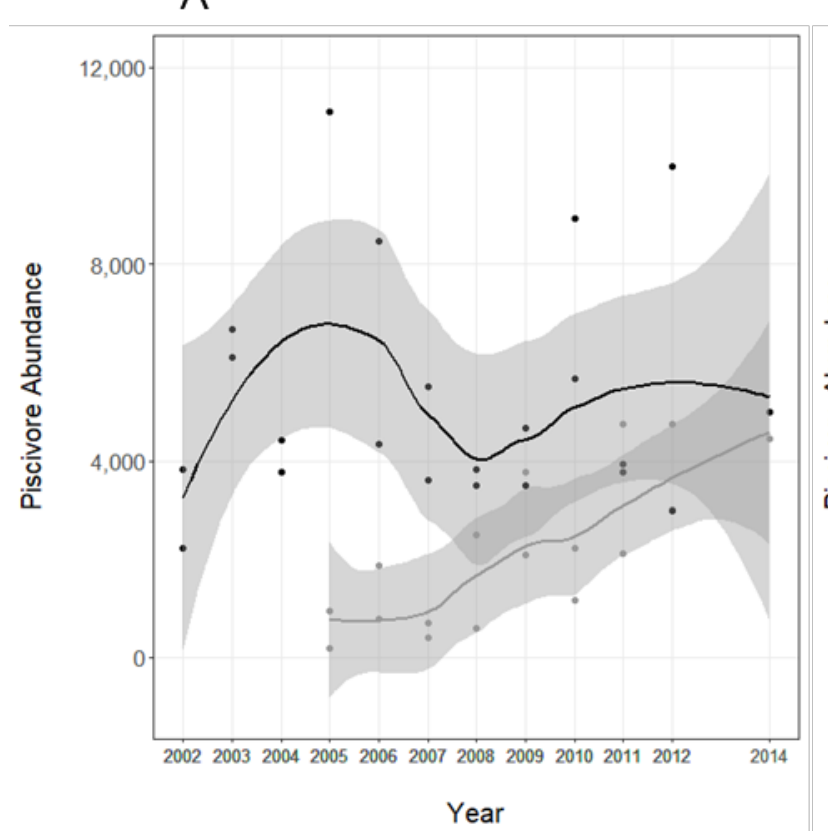

B

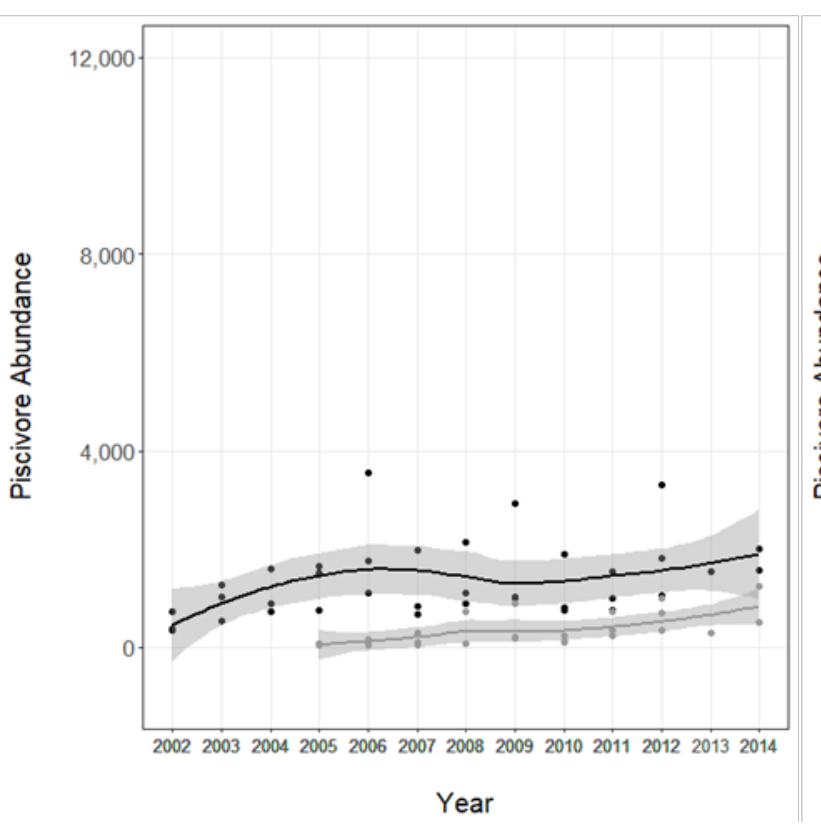

C

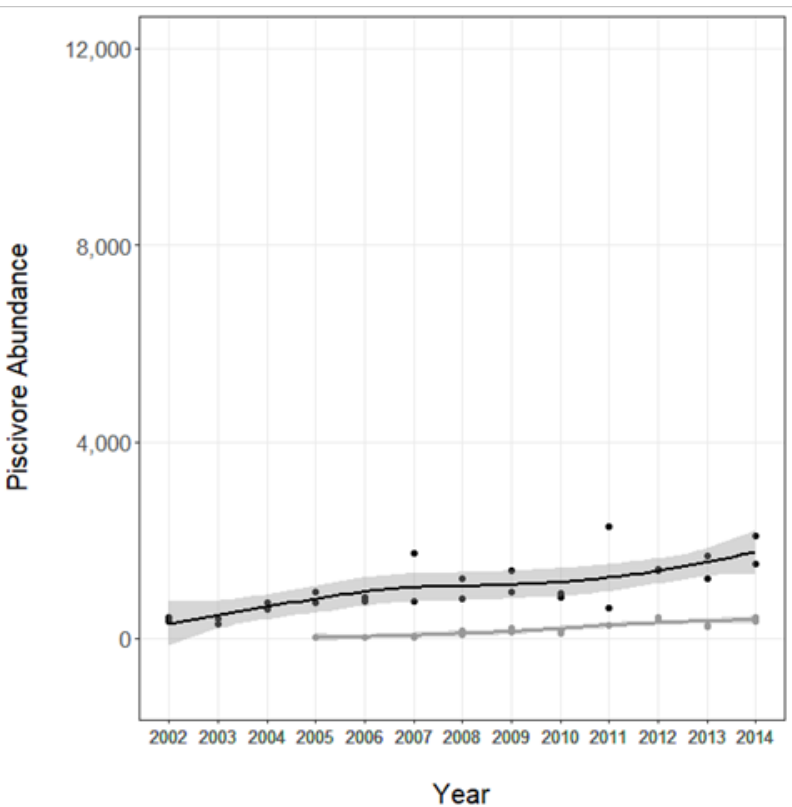

Figure 7. Piscivore population trend during fall (A), winter (B), and spring (C) in South Bay Salt Pond Restoration Project ponds (blue) and active salt production ponds (red), South San Francisco Bay, California. Points represent abundance of each monthly count, where fall includes October and November; winter includes December, January, and February; and spring includes March and April. Trend lines were created using locally weighted scatterplot smoothing in R. Counts were not conducted during fall 2013. Year corresponds to the year of fall, winter 2013 includes January and February 2014, and spring 2013 includes March and April 2014. 
A

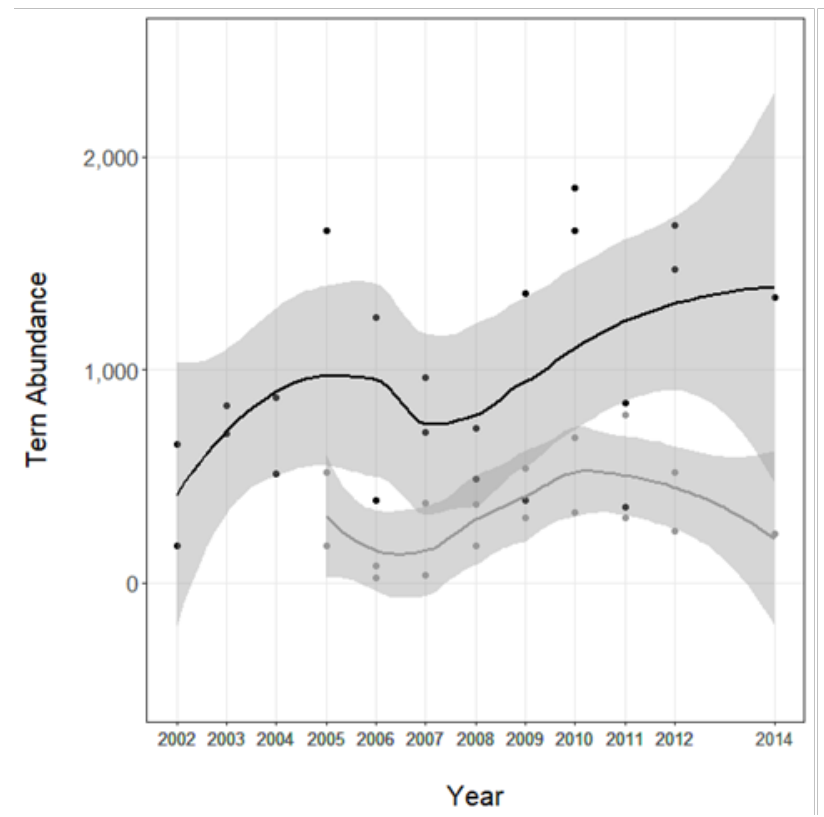

B

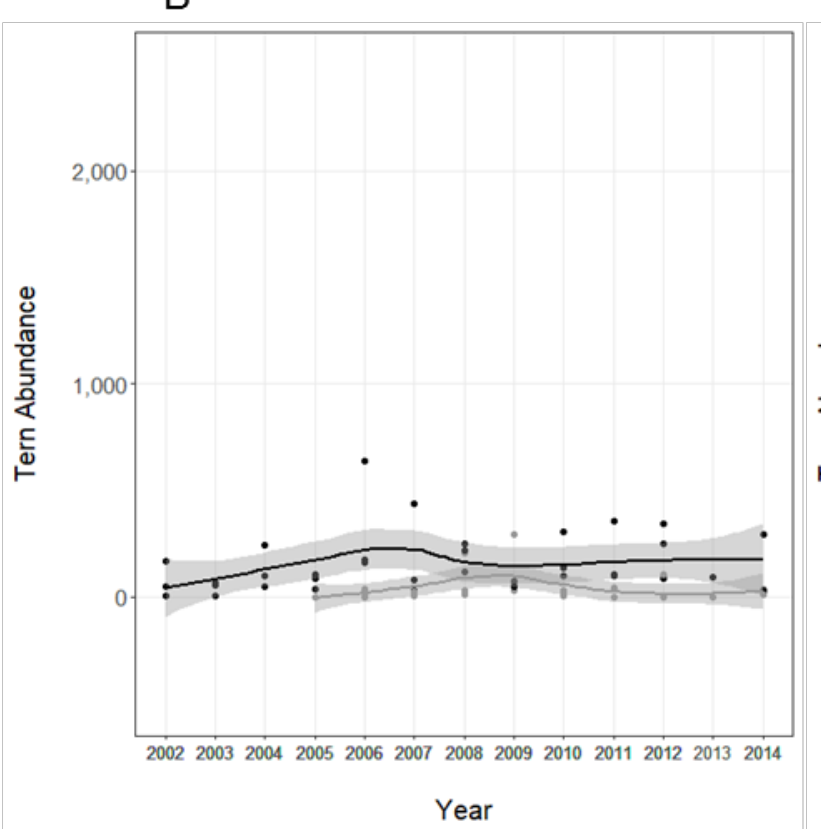

C

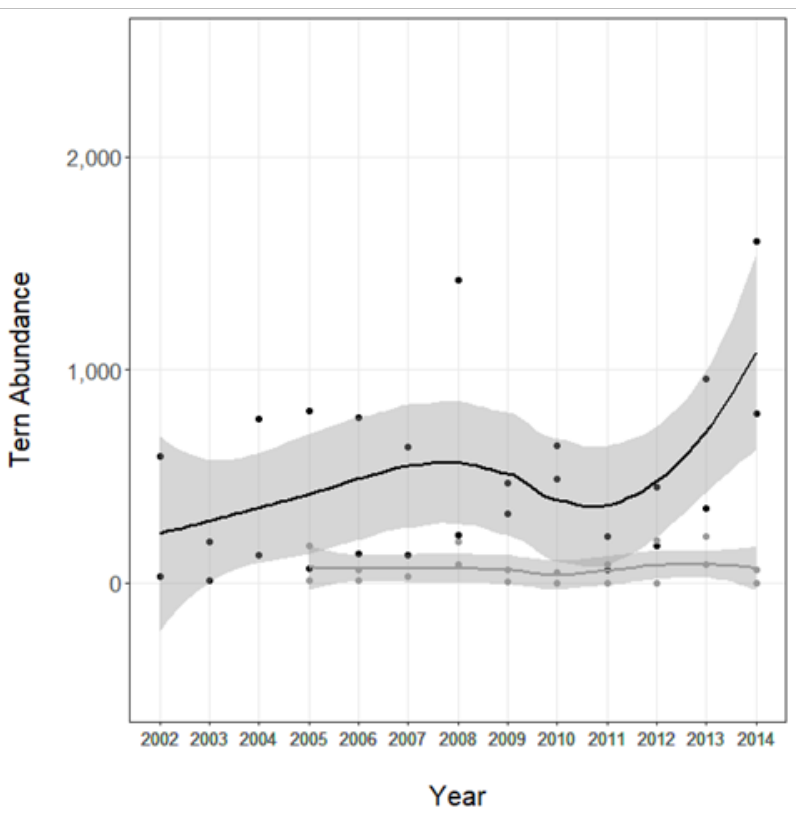

Figure 8. Tern population trend during fall (A), winter (B), and spring (C) in South Bay Salt Pond Restoration Project ponds (dark gray) and active salt production ponds (light gray), South San Francisco Bay, California. Points represent abundance of each monthly count, where fall includes October and November; winter includes December, January, and February; and spring includes March and April. Trend lines were created using locally weighted scatterplot smoothing in R. Counts were not conducted during fall 2013. Year corresponds to the year of fall, winter 2013 includes January and February 2014, and spring 2013 includes March and April 2014. 

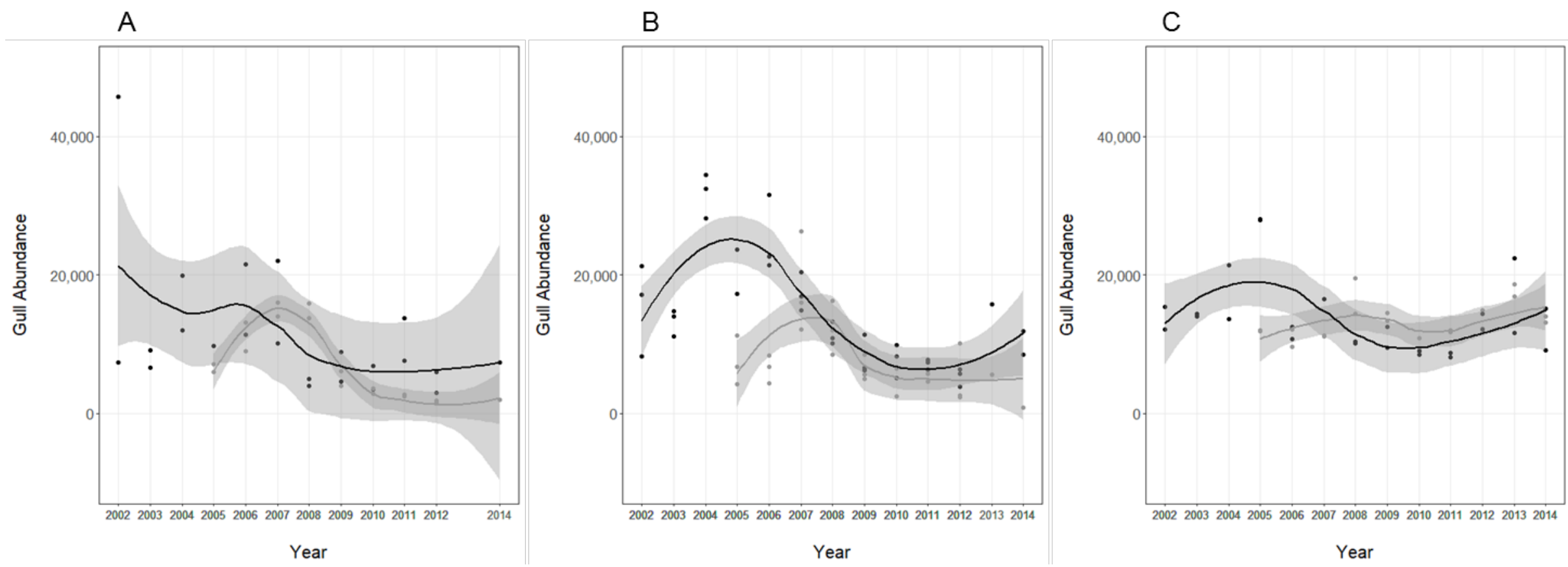

Figure 9. Gull population trend during fall $(A)$, winter $(B)$, and spring $(C)$ in South Bay Salt Pond Restoration Project ponds (dark gray) and active salt production ponds (light gray), South San Francisco Bay, California. Points represent abundance of each monthly count, where fall includes October and November; winter includes December, January, and February; and spring includes March and April. Trend lines were created using locally weighted scatterplot smoothing in R. Counts were not conducted during fall 2013. Year corresponds to the year of fall, winter 2013 includes January and February 2014, and spring 2013 includes March and April 2014 
A

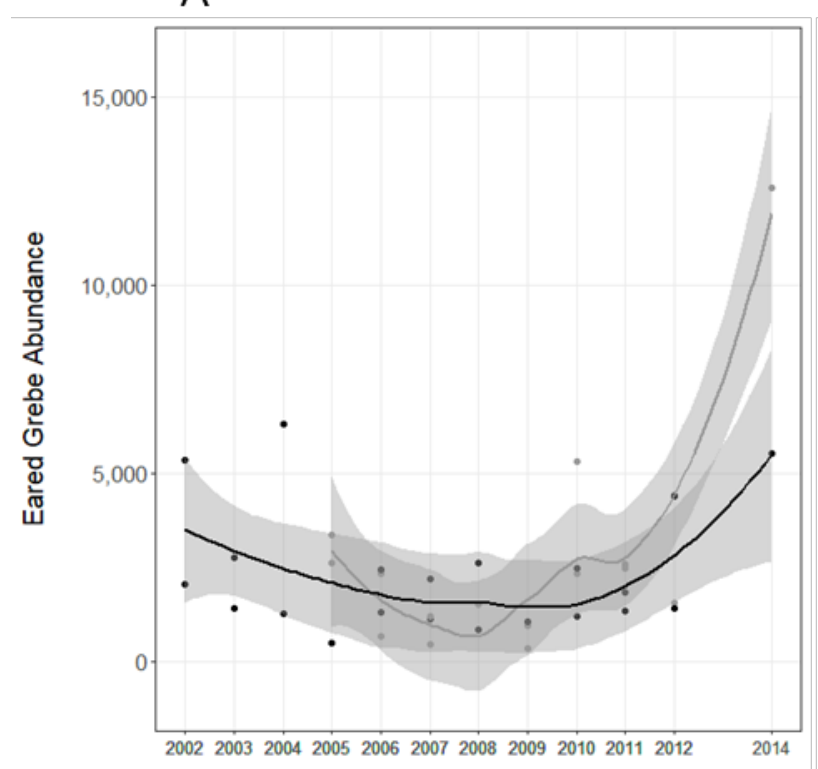

Year
B

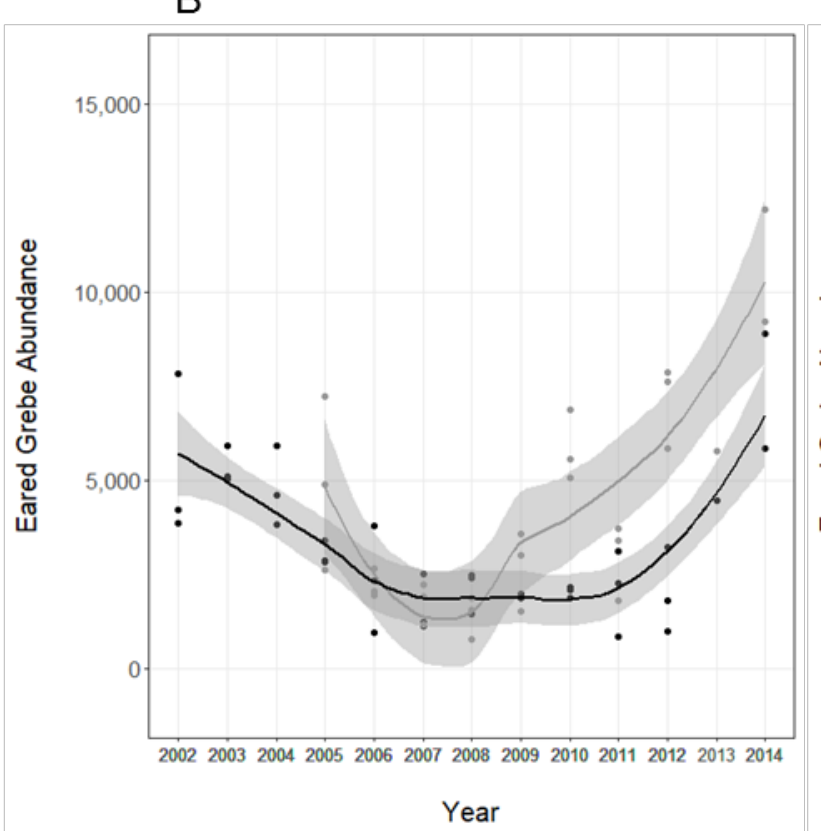

C

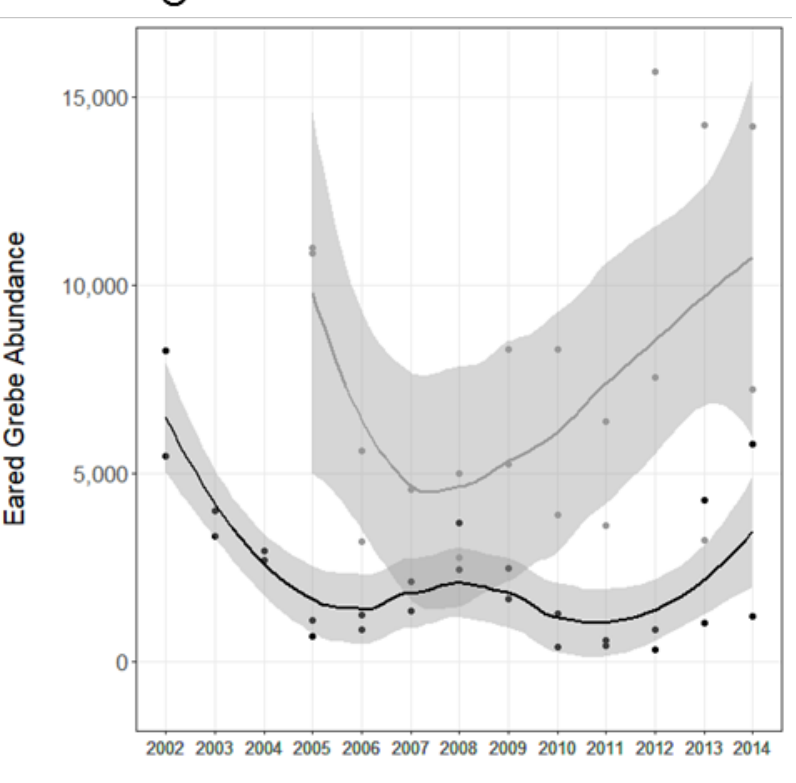

Year

Figure 10. Eared grebe population trend during fall $(\mathrm{A})$, winter $(\mathrm{B})$, and spring $(\mathrm{C})$ in South Bay Salt Pond Restoration Project ponds (dark gray) and active salt production ponds (light gray), South San Francisco Bay, California. Points represent abundance of each monthly count, where fall includes October and November; winter includes December, January, and February; and spring includes March and April. Trend lines were created using locally weighted scatterplot smoothing in R. Counts were not conducted during fall 2013. Year corresponds to the year of fall, winter 2013 includes January and February 2014, and spring 2013 includes March and April 2014. 
A

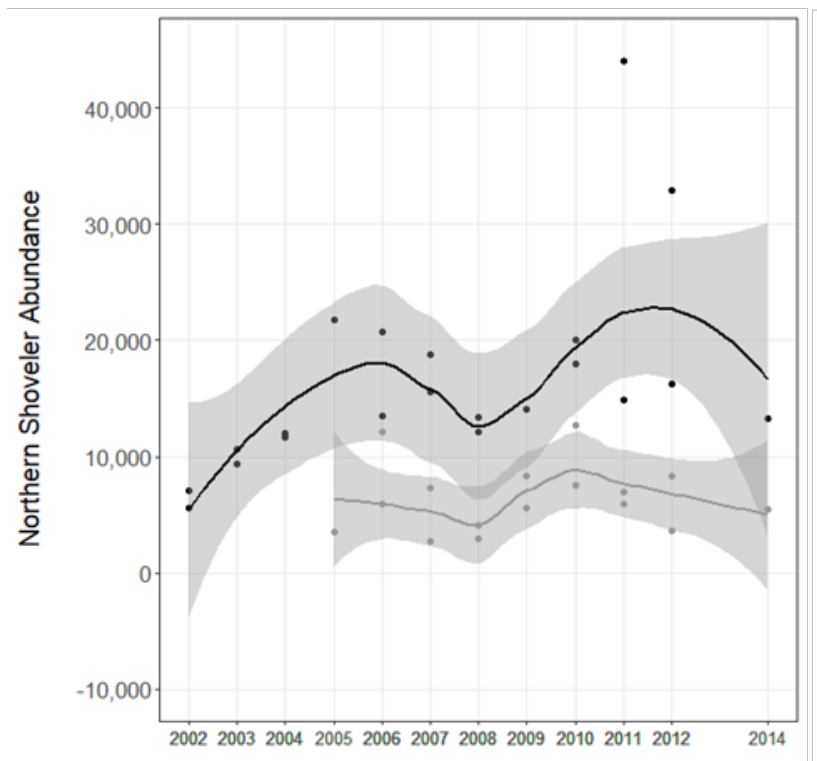

Year
B

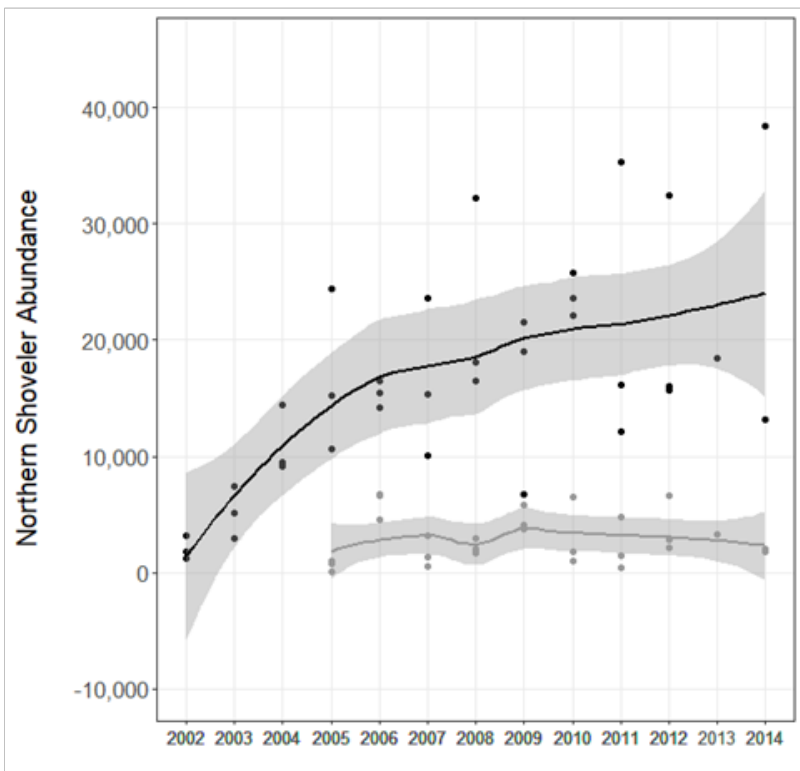

Year
C

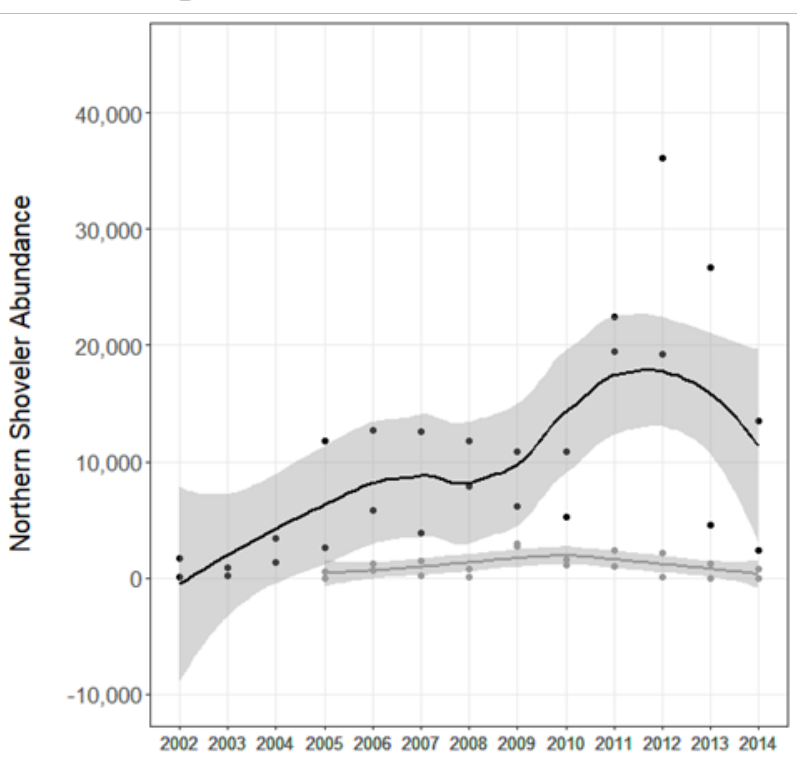

Year

Figure 11. Northern shoveler population trend during fall (A), winter (B), and spring (C) in South Bay Salt Pond Restoration Project ponds (dark gray) and active salt production ponds (light gray), South San Francisco Bay, California. Points represent abundance of each monthly count, where fall includes October and November; winter includes December, January, and February; and spring includes March and April. Trend lines were created using locally weighted scatterplot smoothing in R. Counts were not conducted during fall 2013. Year corresponds to the year of fall, winter 2013 includes January and February 2014, and spring 2013 includes March and April 2014. 
A

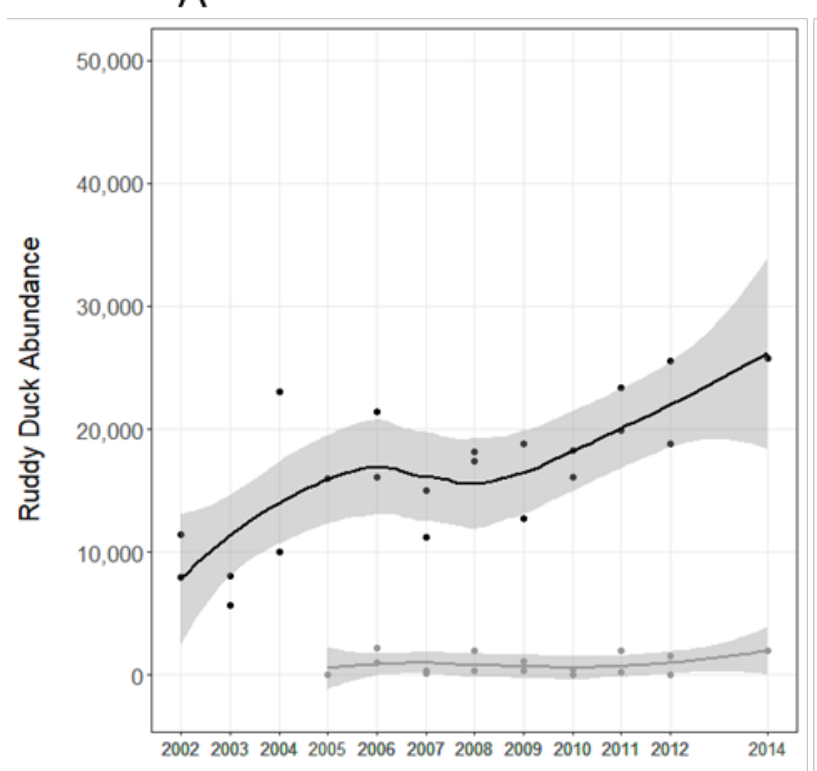

Year
B

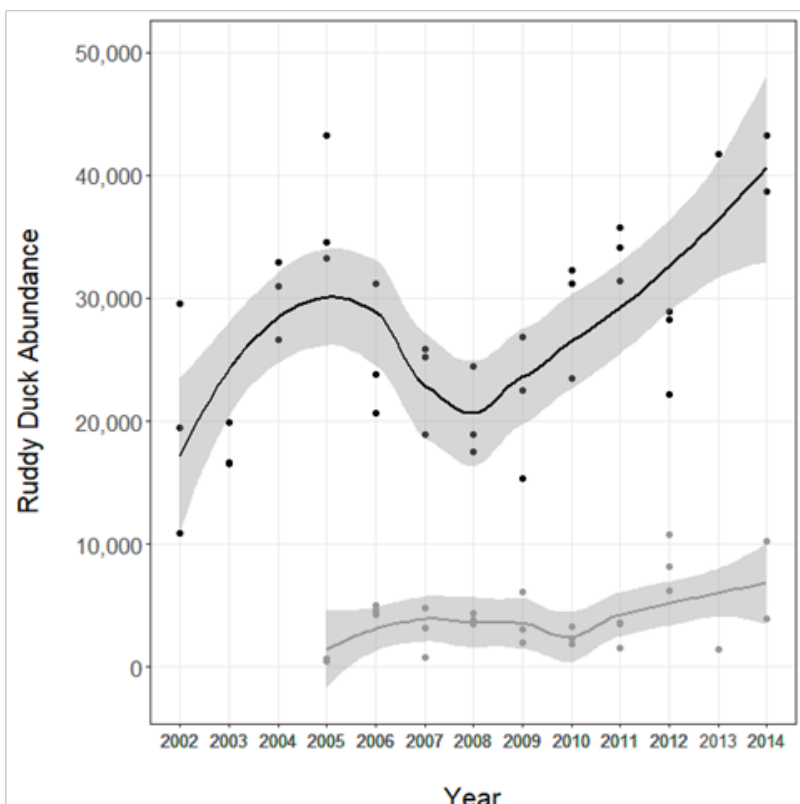

C

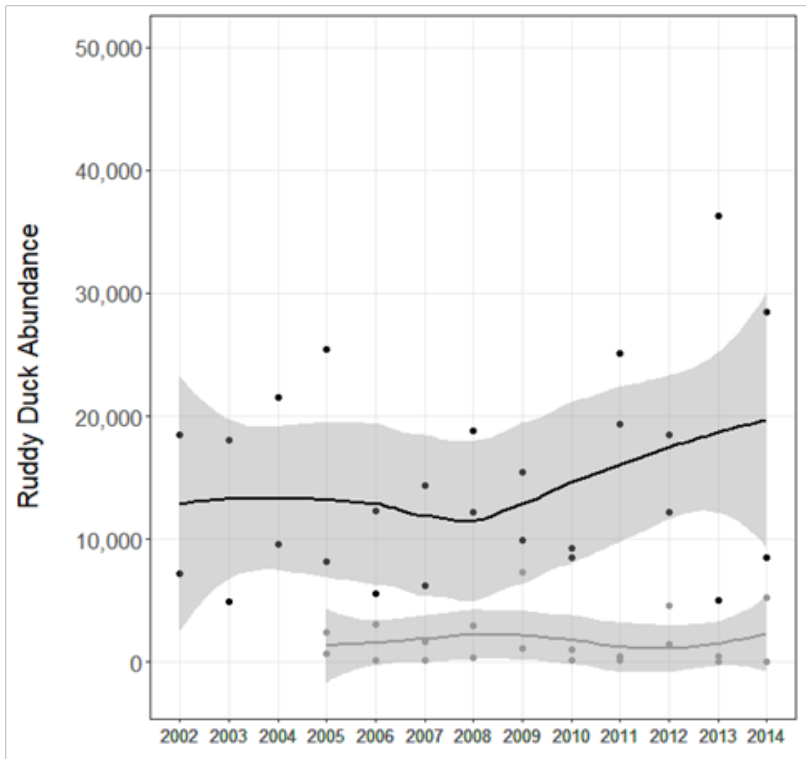

Year

Figure 12. Ruddy duck population trend during fall $(A)$, winter $(B)$, and spring $(C)$ in South Bay Salt Pond Restoration Project ponds (dark gray) and active salt production ponds (light gray), South San Francisco Bay, California. Points represent abundance of each monthly count, where fall includes October and November; winter includes December, January, and February; and spring includes March and April. Trend lines were created using locally weighted scatterplot smoothing in R. Counts were not conducted during fall 2013. Year corresponds to the year of fall, winter 2013 includes January and February 2014, and spring 2013 includes March and April 2014. 
A

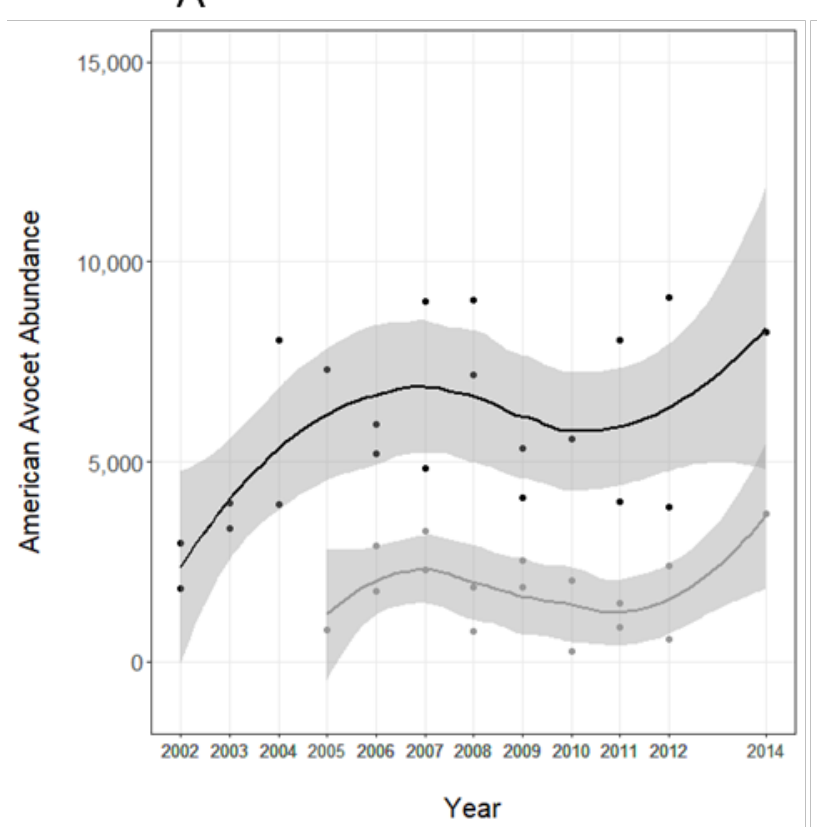

B

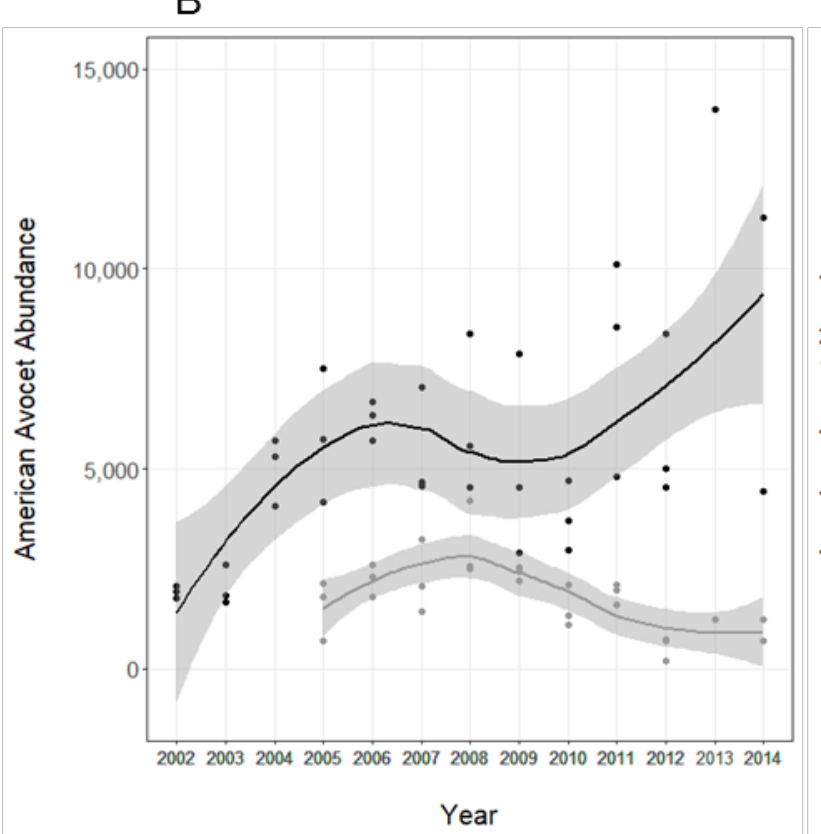

C

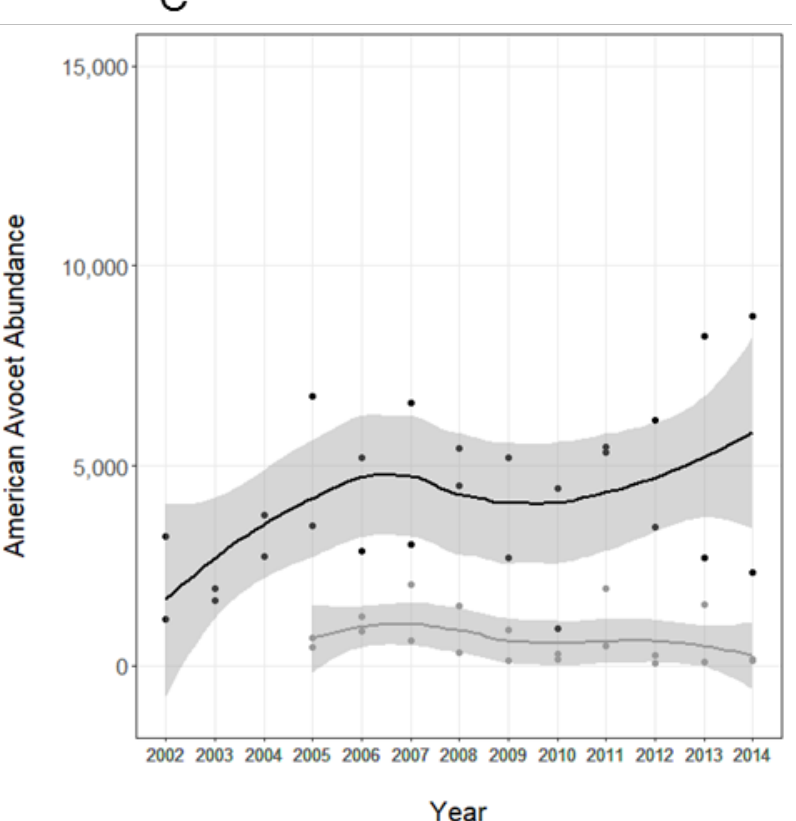

Figure 13. American avocet population trend during fall (A), winter (B), and spring (C) in South Bay Salt Pond Restoration Project ponds (dark gray) and active salt production ponds (light gray), South San Francisco Bay, California. Points represent abundance of each monthly count, where fall includes October and November; winter includes December, January, and February; and spring includes March and April. Trend lines were created using locally weighted scatterplot smoothing in R. Counts were not conducted during fall 2013. Year corresponds to the year of fall, winter 2013 includes January and February 2014, and spring 2013 includes March and April 2014. 
A

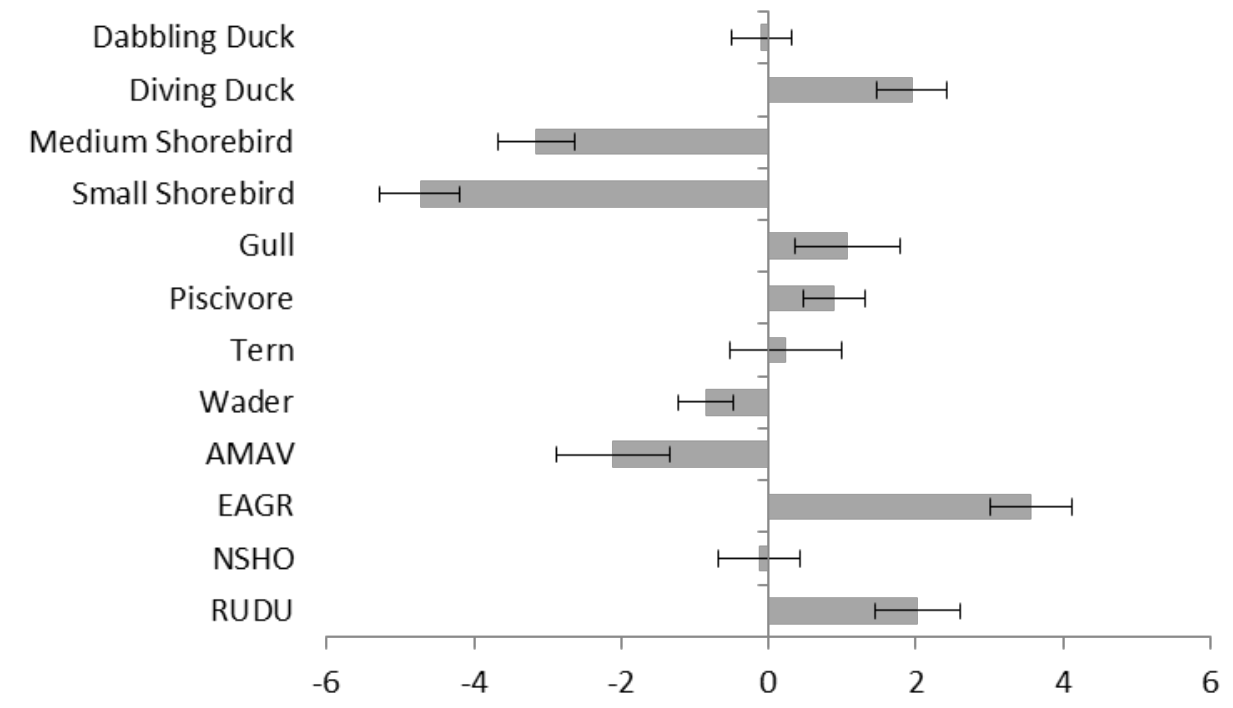

B

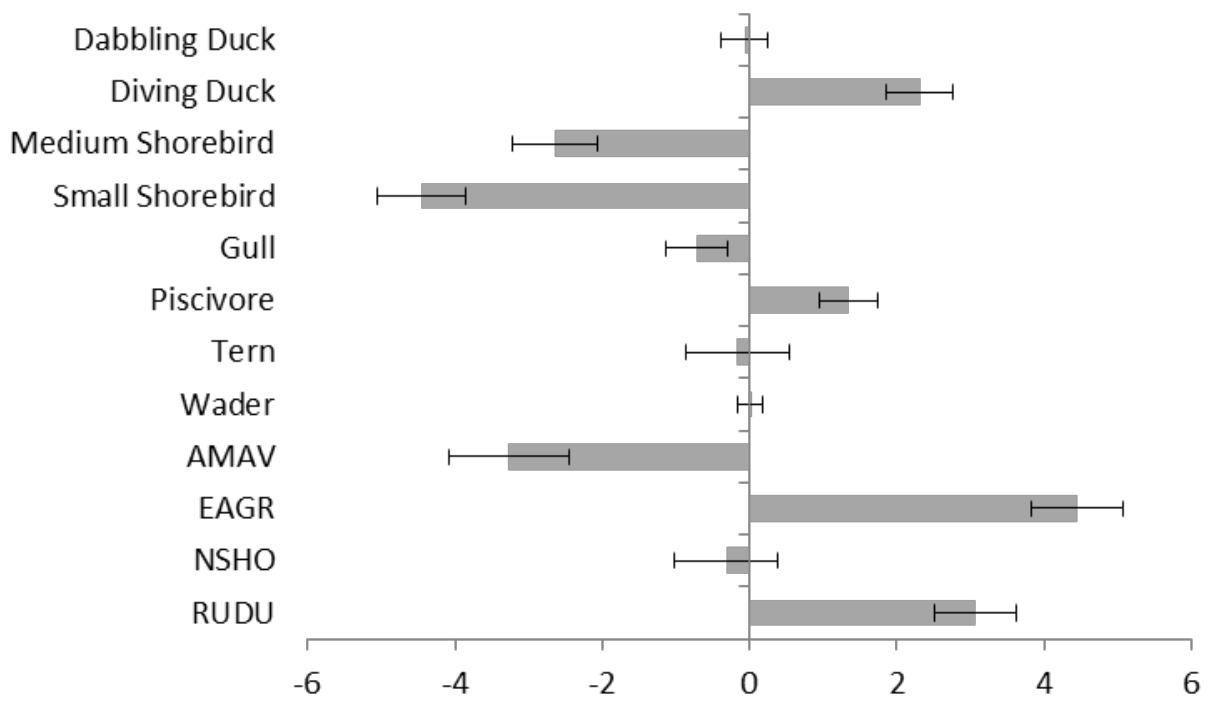

Figure 14. Model-averaged coefficients ( \pm 95 -percent confidence interval $[\mathrm{CI}])$ of pond water depth for $(A)$ foraging and $(B)$ roosting waterbirds at the pond scale in restoration ponds, South San Francisco Bay, California. Coefficients were considered significant only if the 95-percent $\mathrm{Cl}$ does not overlap zero. Bar length indicates the coefficient effect size and is comparable across species and guilds. AMAV, American avocet; EAGR, eared grebe; NSHO, northern shoveler; RUDU, ruddy duck. 

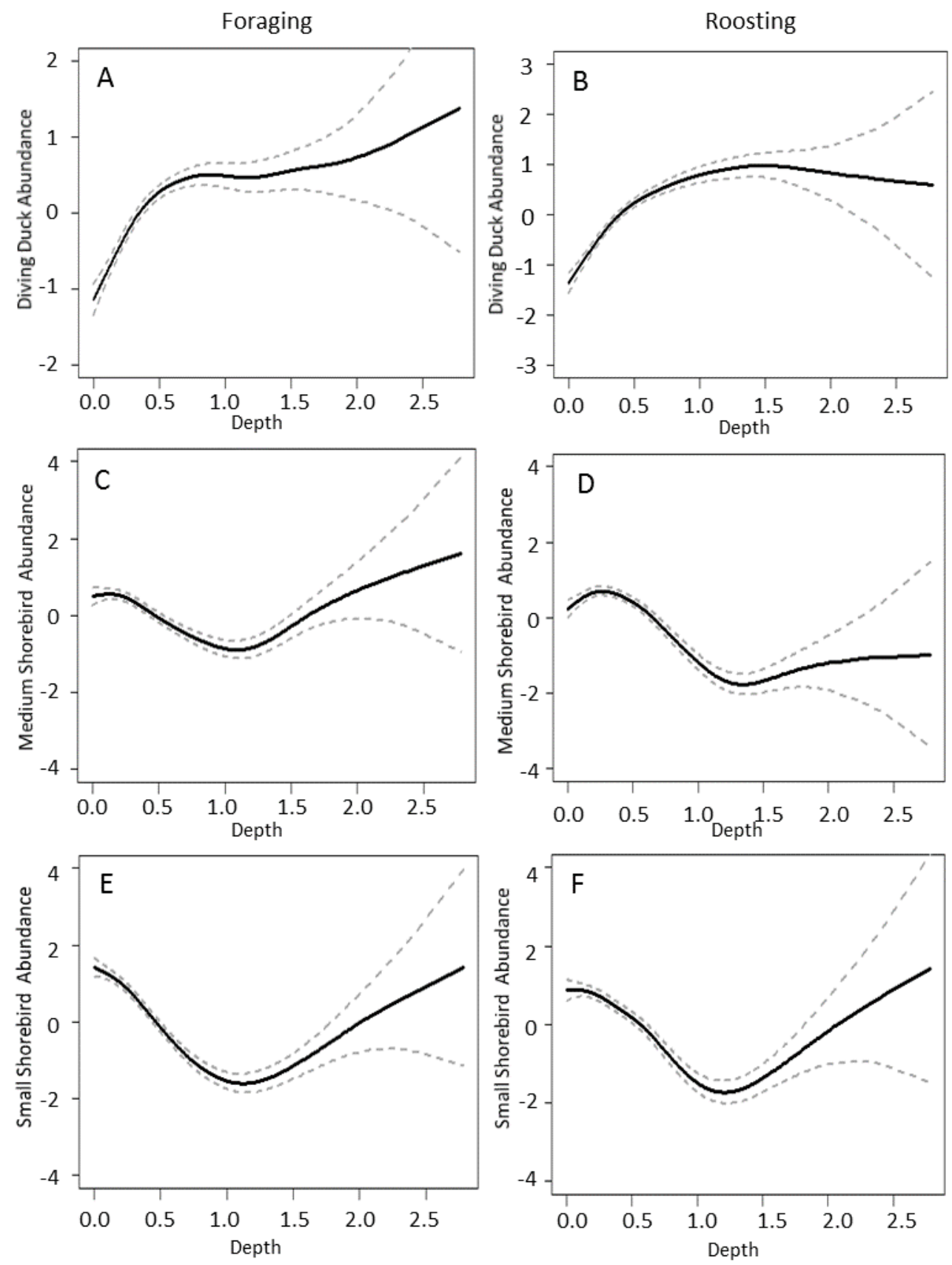

Figure 15. Diving duck (A-B), medium (C-D) and small shorebird (E-F), gull (G-H), piscivore (I-J) and wader $(K)$ abundance response to water depth (in meters) from generalized additive mixed models at the pond scale in restoration ponds, South San Francisco Bay, California. Dashed lines indicate the 95-percent confidence interval. Note: $Y$-axis differs among figures. 

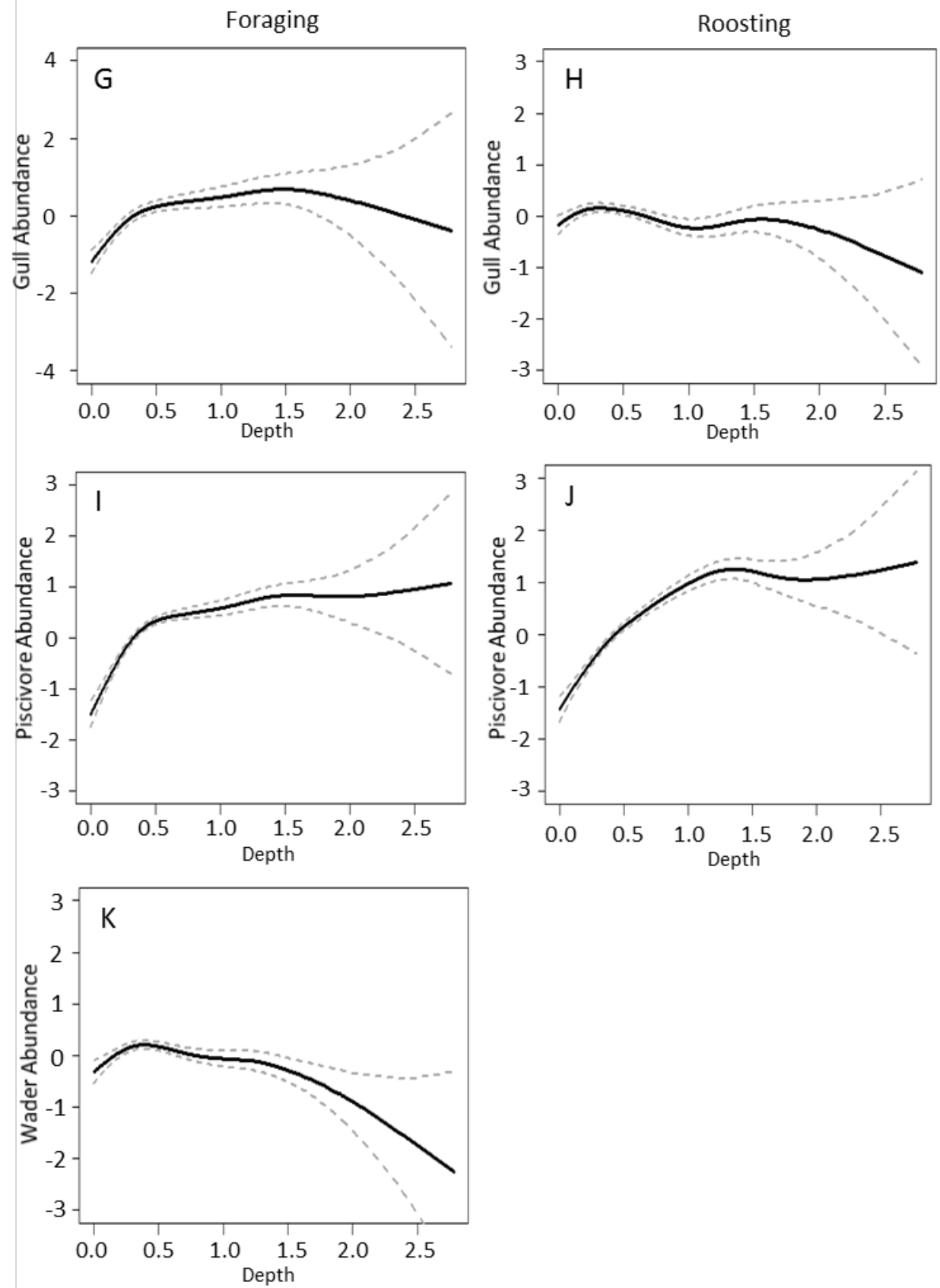

Figure 15. - Continued 
A

Dabbling Duck

Diving Duck

Medium Shorebird

Small Shorebird

Gull

Piscivore

Tern

Wader

AMAV

EAGR

NSHO

RUDU

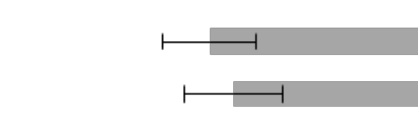

. 

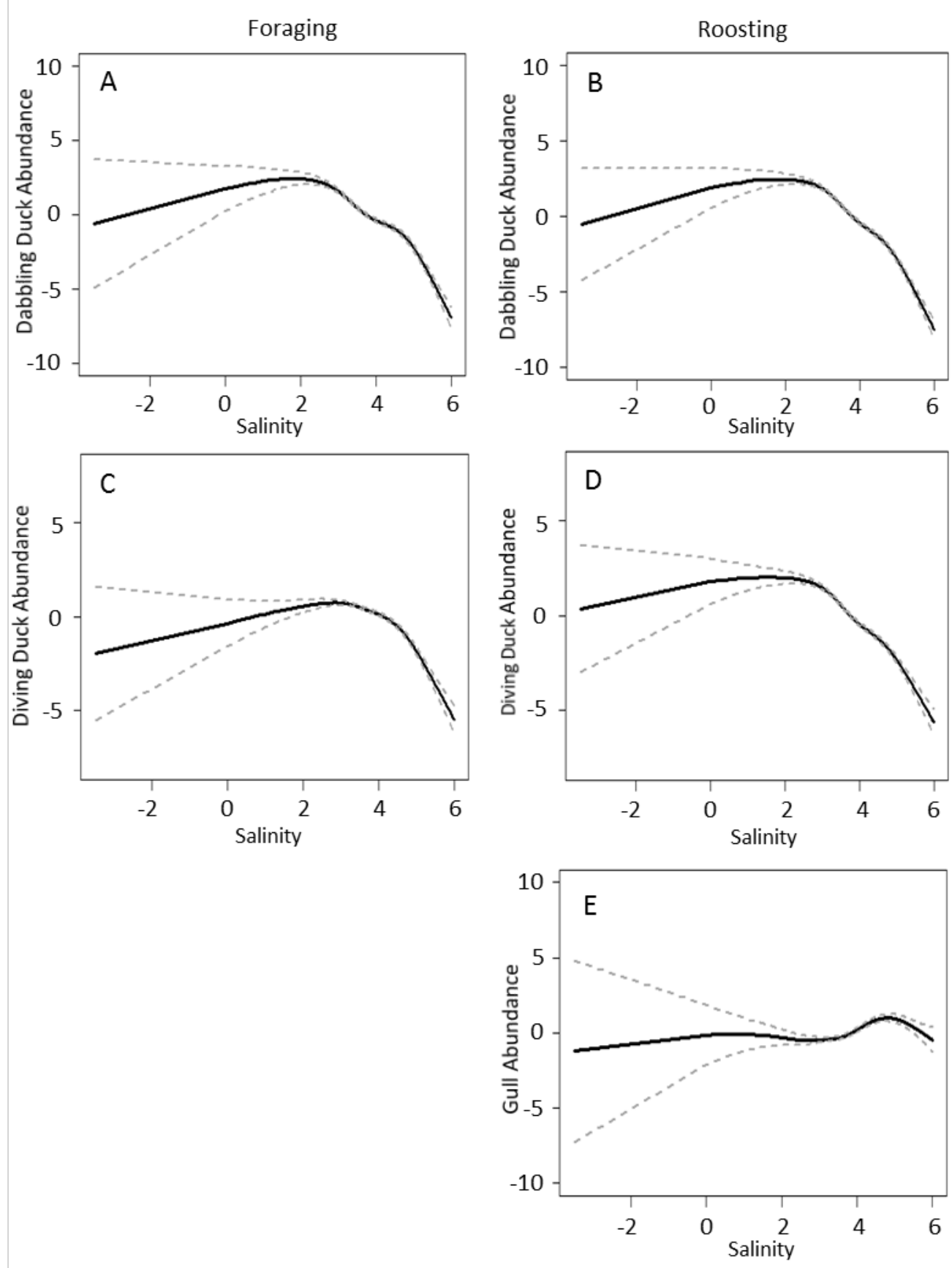

Figure 17. Dabbling duck $(A-B)$, diving duck $(C-D)$, gull $(E)$, piscivore $(F-G)$, tern $(H-I)$,and wader $(J-K)$ abundance response to salinity (in $\log$ [parts per thousand]) from generalized additive mixed models at the pond scale in restoration ponds, South San Francisco Bay, California. Dashed lines indicate the 95-percent confidence interval. Note: Y-axis differs among figures. 

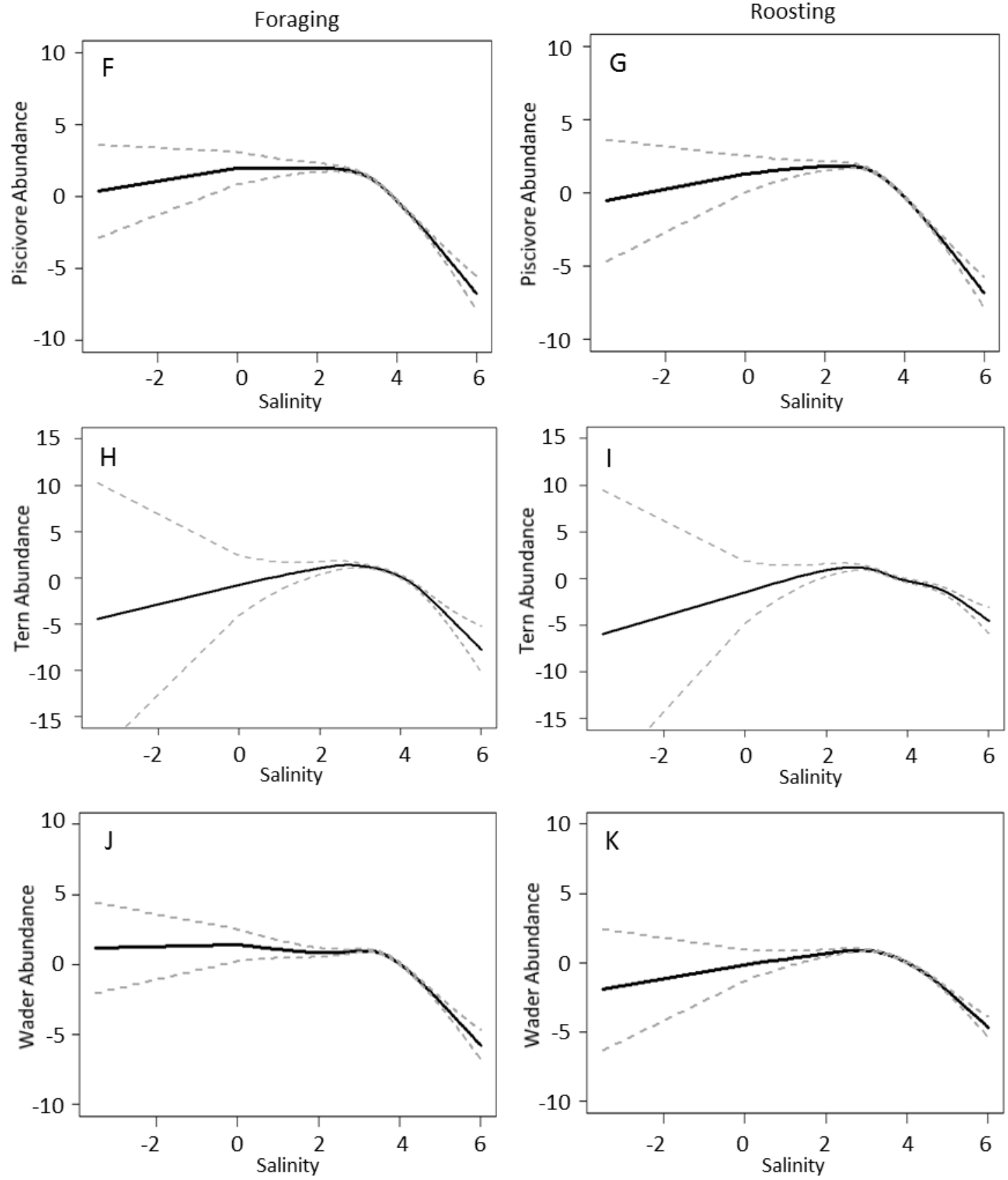

Figure 17.-Continued. 
A

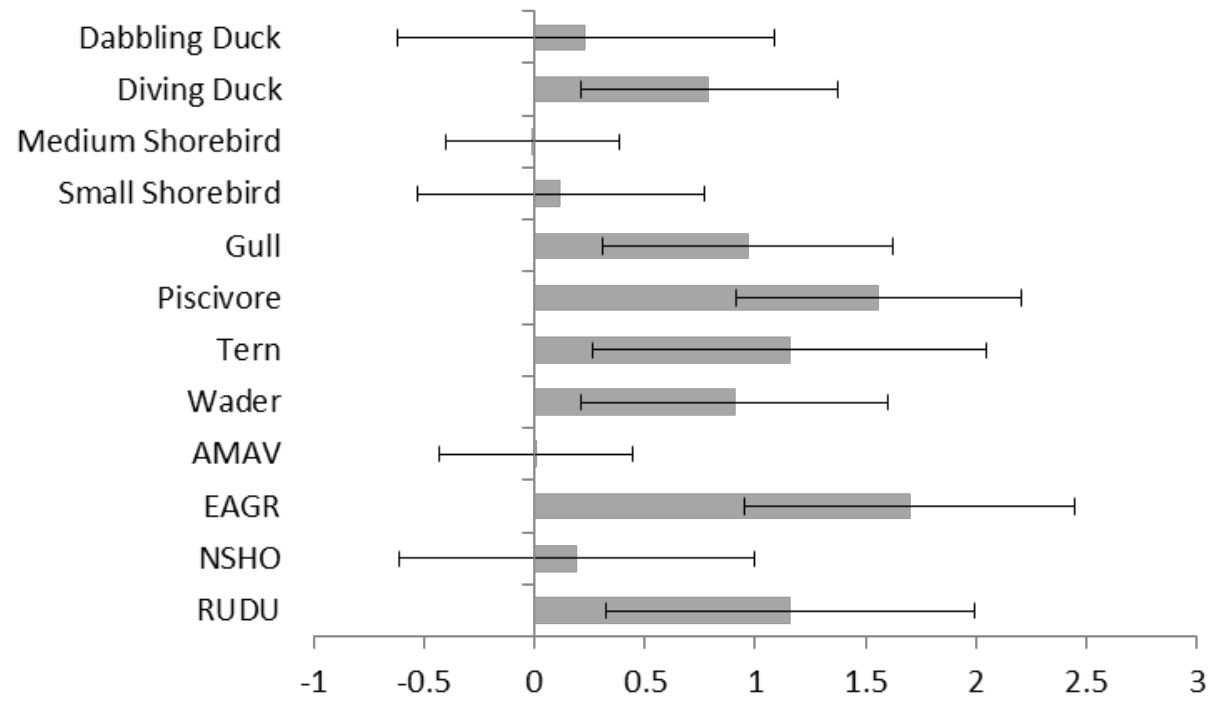

B

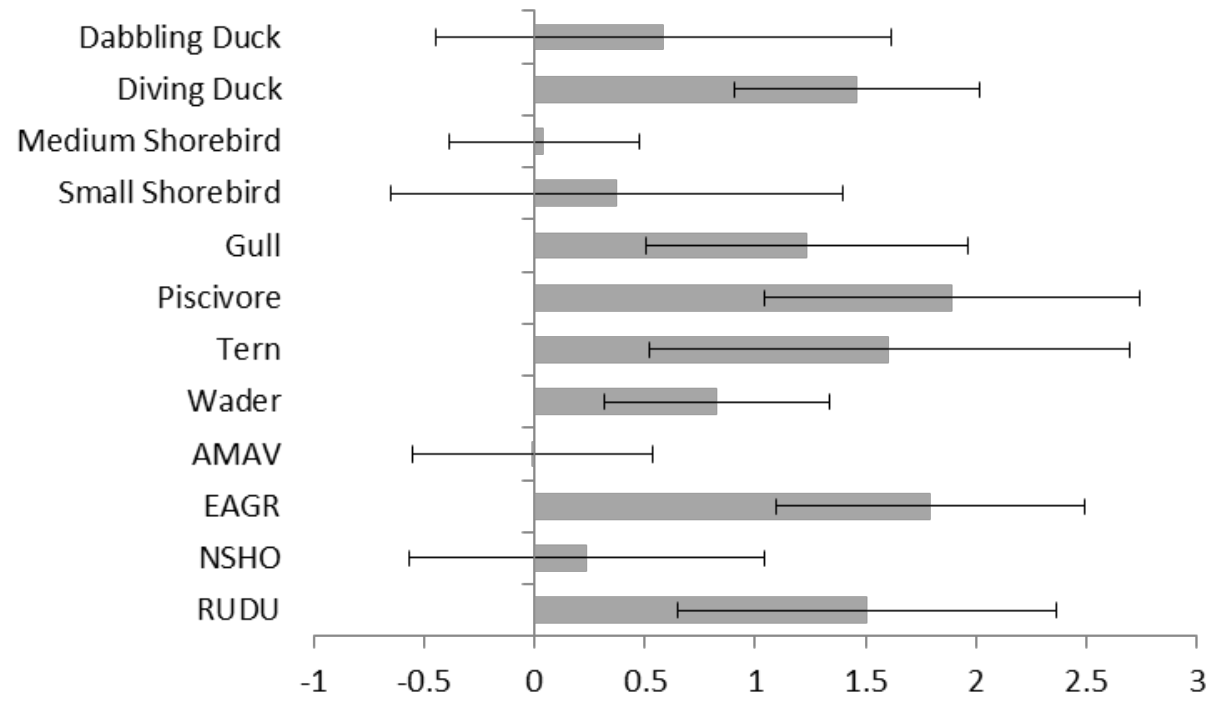

Figure 18. Model-averaged coefficients ( \pm 95 -percent confidence interval $[\mathrm{Cl}])$ of pond area for $(\mathrm{A})$ foraging and (B) roosting waterbirds at the pond scale in restoration ponds, South San Francisco Bay, California. Coefficients are considered significant only if the 95-percent $\mathrm{Cl}$ does not overlap zero. Bar length indicates the coefficient effect size and is comparable across species and guilds. AMAV, American avocet; EAGR, eared grebe; NSHO, northern shoveler; RUDU, ruddy duck. 

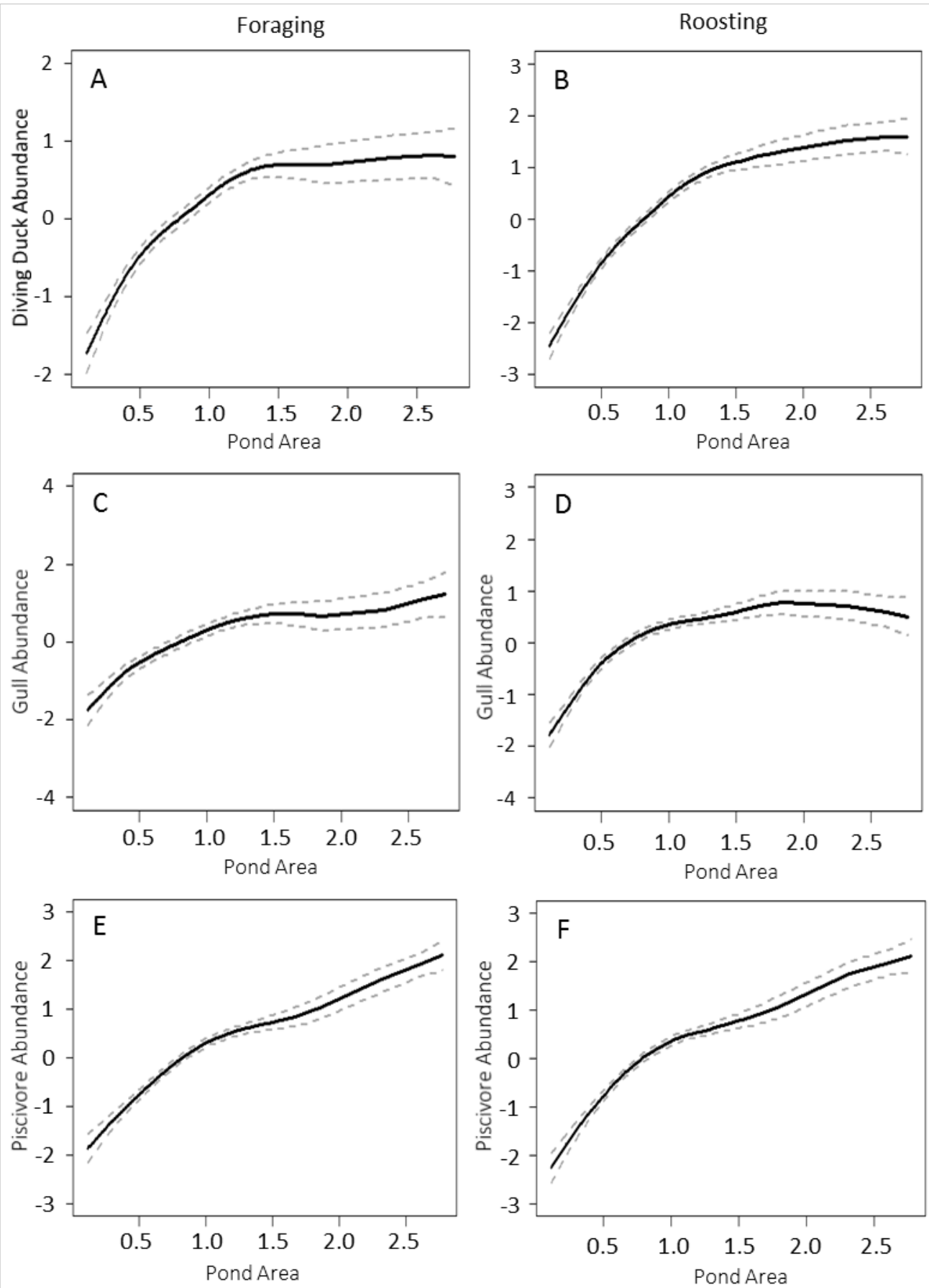

Figure 19. Diving duck (A-B), gull (C-D), piscivore $(E-F)$, tern $(G-H)$, and wader $(I-J)$ abundance response to pond area (in square kilometers) from generalized additive mixed models at the pond scale in restoration ponds, South San Francisco Bay, California. Dashed lines indicate the 95-percent confidence interval. Note: Y-axis differs among figures. 

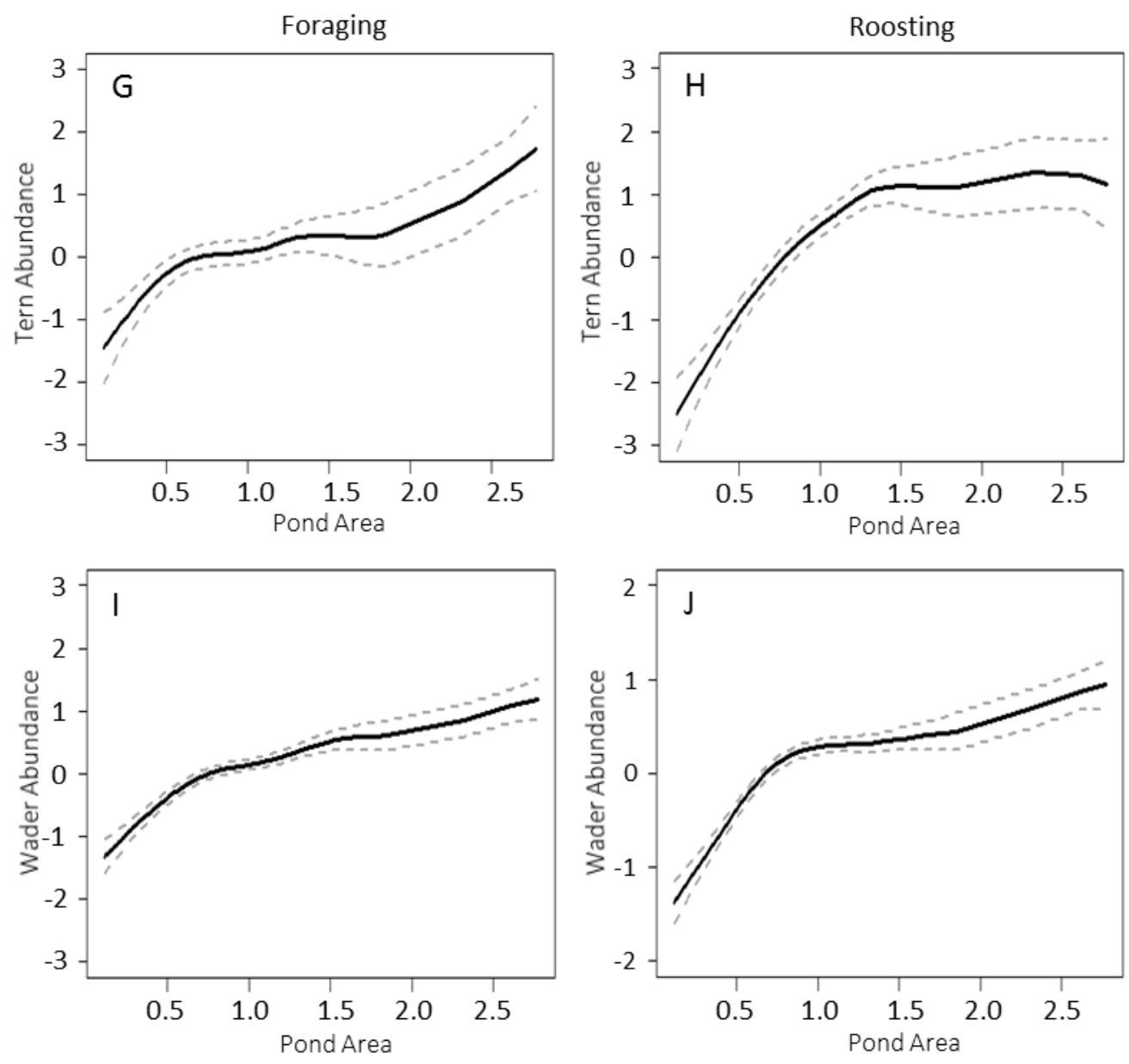

Figure 19. - Continued. 
A

Dabbling Duck

Diving Duck

Medium Shorebird

Small Shorebird

Gull

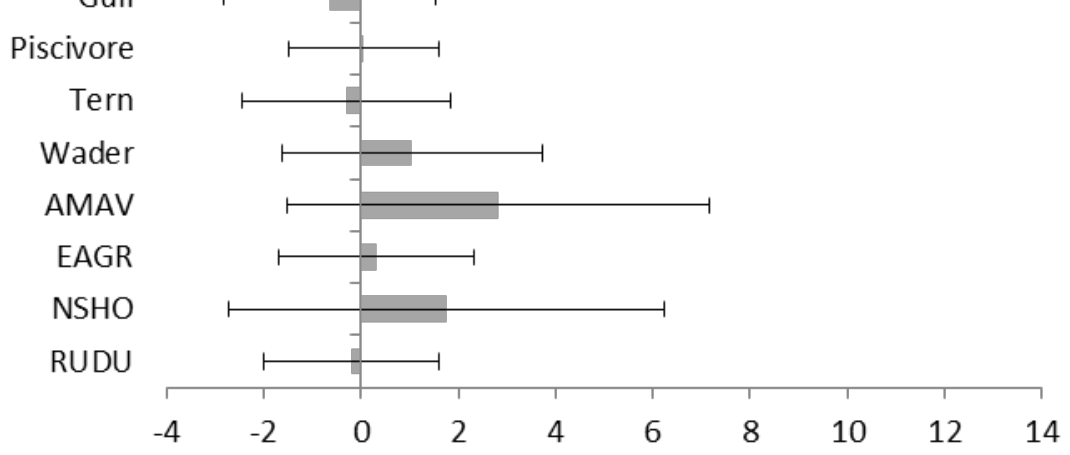

B

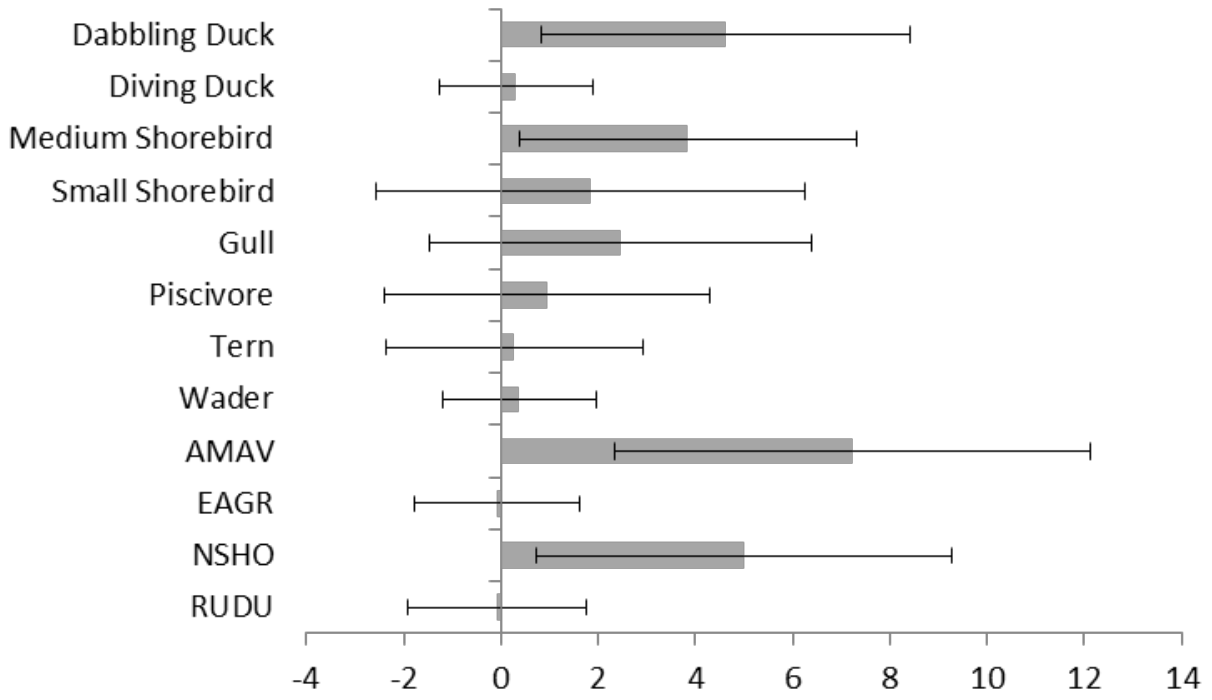

Figure 20. Model-averaged coefficients ( \pm 95 -percent confidence interval $[\mathrm{Cl}])$ of pond topography for $(\mathrm{A})$ foraging and (B) roosting waterbirds at the pond scale in restoration ponds, South San Francisco Bay, California. Coefficients are considered significant only if the 95-percent $\mathrm{Cl}$ does not overlap zero. Bar length indicates the coefficient effect size and is comparable across species and guilds. AMAV, American avocet; EAGR, eared grebe; NSHO, northern shoveler; RUDU, ruddy duck. 

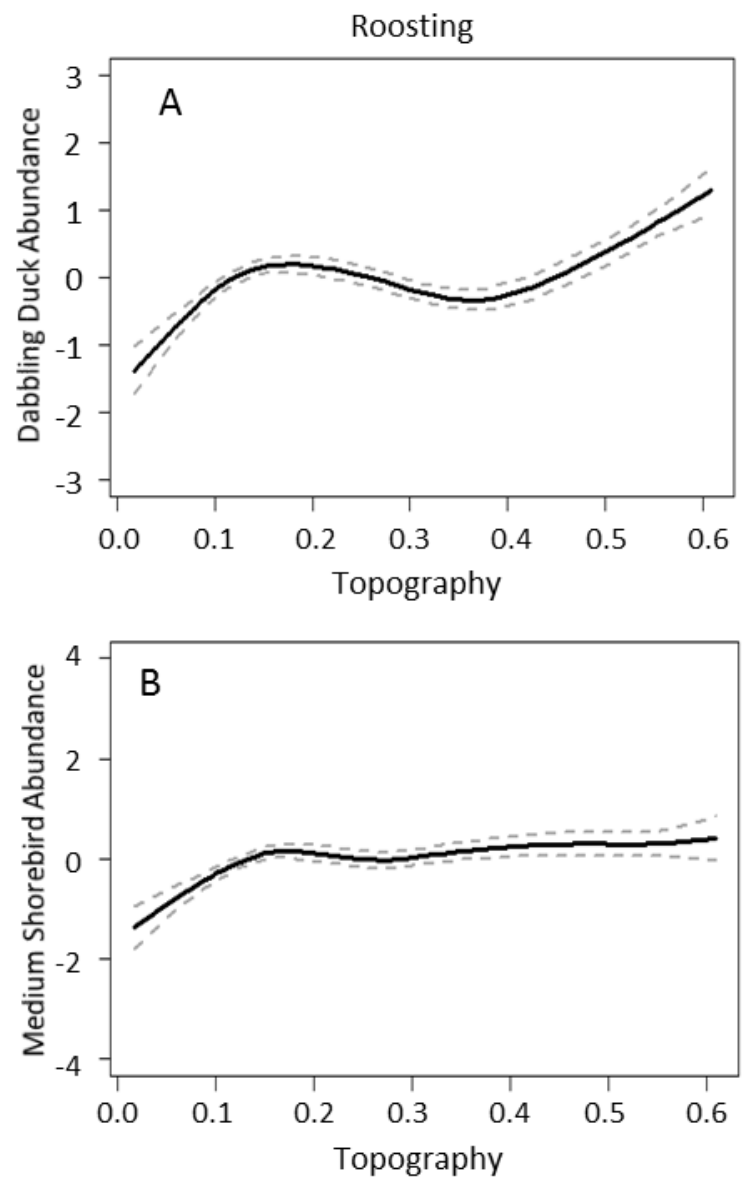

Figure 21. Roosting dabbling duck (A) and roosting medium shorebird (B) abundance response to pond topography (in meters) from generalized additive mixed models at the pond scale in restoration ponds, South San Francisco Bay, California. Dashed lines indicate the 95-percent confidence interval. Note: Y-axis differs between figures. 
A

Dabbling Duck

Diving Duck

Medium Shorebird

Small Shorebird

Gull

Piscivore

Tern

Wader

AMAV

EAGR

NSHO

RUDU

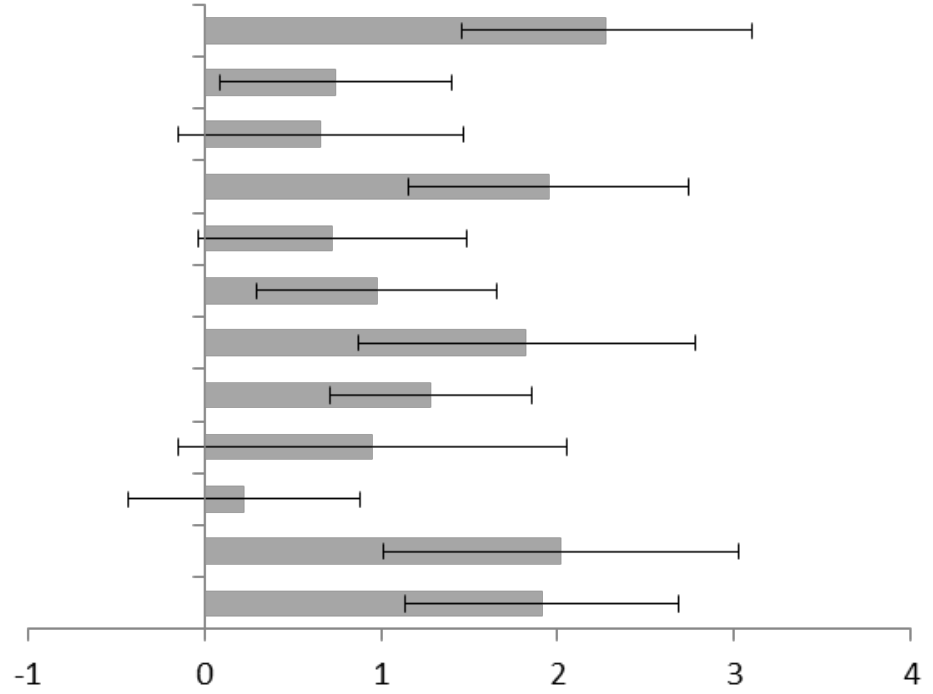

B

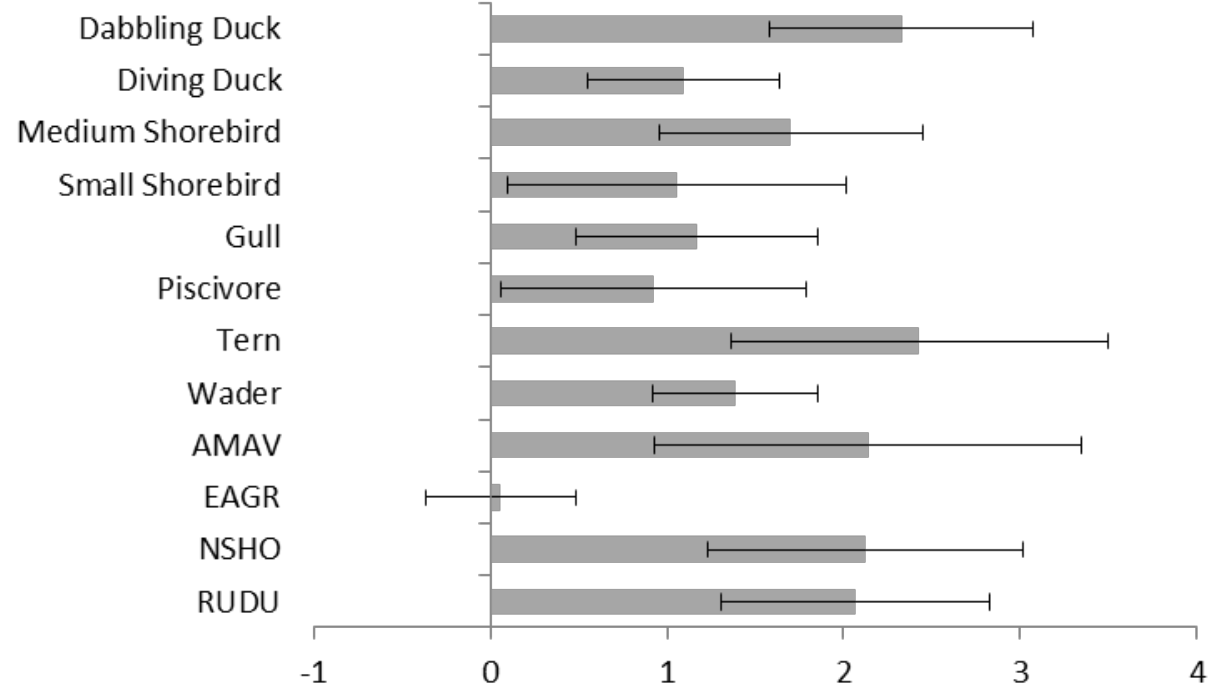

Figure 22. Model-averaged coefficients ( \pm 95 -percent confidence interval $[\mathrm{Cl}]$ ) of islands for $(\mathrm{A})$ foraging and $(\mathrm{B})$ roosting waterbirds at the pond scale in restoration ponds, South San Francisco Bay, California. Coefficients are considered significant only if the 95-percent $\mathrm{Cl}$ does not overlap zero. Bar length indicates the coefficient effect size and is comparable across species and guilds. AMAV, American avocet; EAGR, eared grebe; NSHO, northern shoveler; RUDU, ruddy duck. 
A

Dabbling Duck

Diving Duck

Medium Shorebird

Small Shorebird

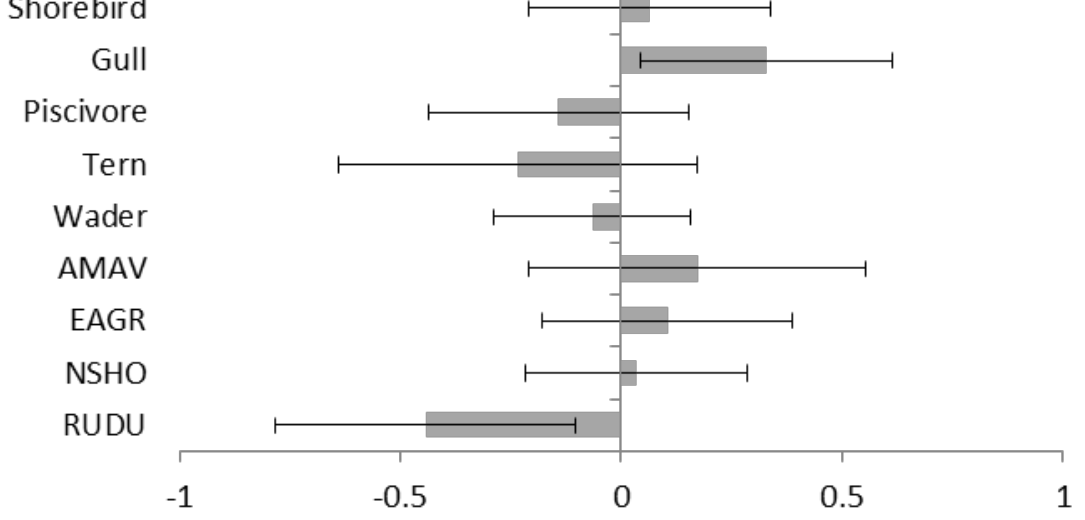

B

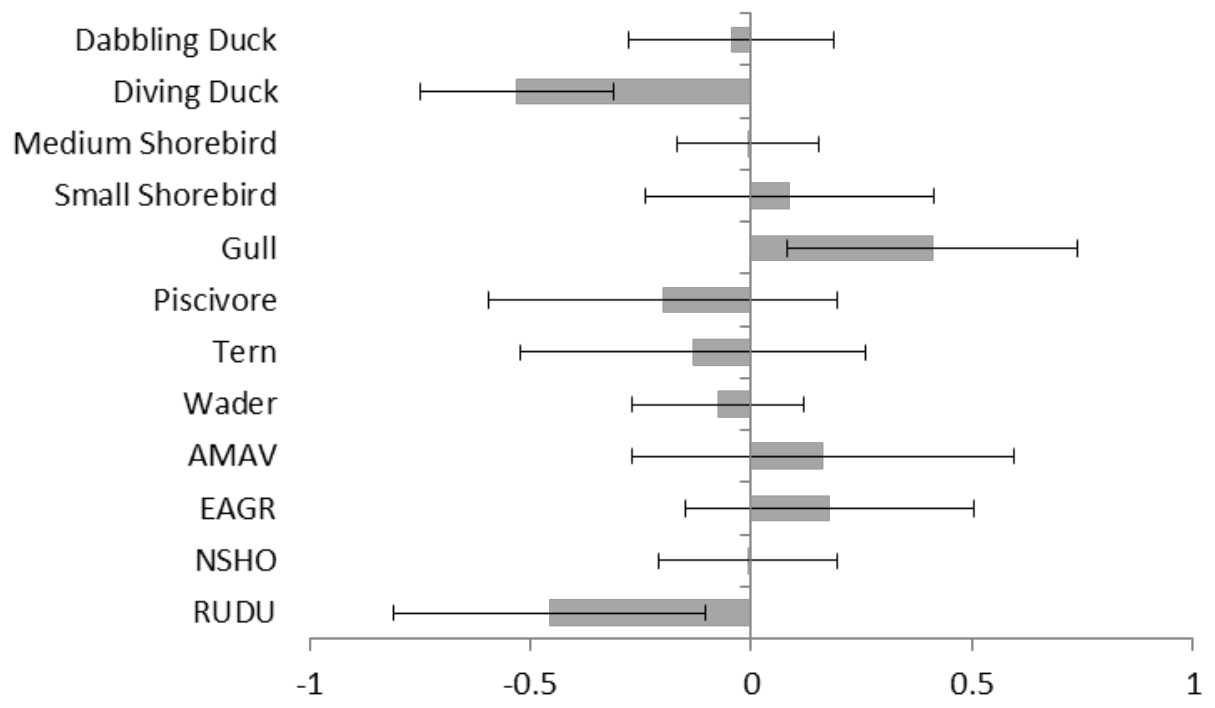

Figure 23. Model-averaged coefficients ( \pm 95 -percent confidence interval $[\mathrm{Cl}]$ ) of distance to San Francisco Bay for (A) foraging and (B) roosting waterbirds at the pond scale in restoration ponds, South San Francisco Bay, California. Coefficients are considered significant only if the 95-percent $\mathrm{Cl}$ does not overlap zero. Bar length indicates the coefficient effect size and is comparable across species and guilds. AMAV, American avocet; EAGR, eared grebe; NSHO, northern shoveler; RUDU, ruddy duck. 

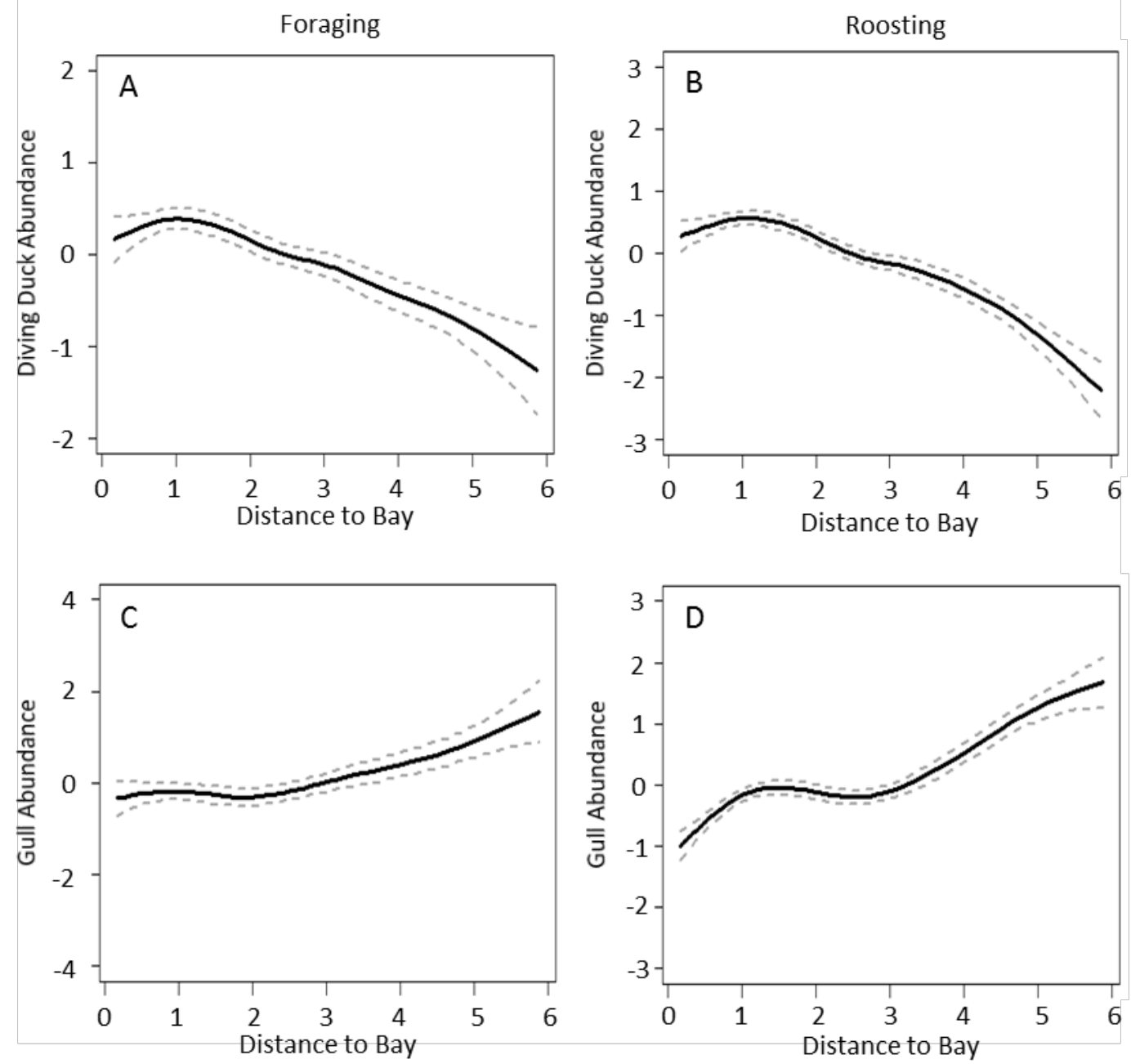

Figure 24. Diving duck ( $A-B)$ and gull ( $C-D)$ abundance response to pond distance (in kilometers) to San Francisco Bay from generalized additive mixed models at the pond scale in restoration ponds, South San Francisco Bay, California. Dashed lines indicate the 95-percent confidence interval. Note: $Y$-axis differs among figures. 


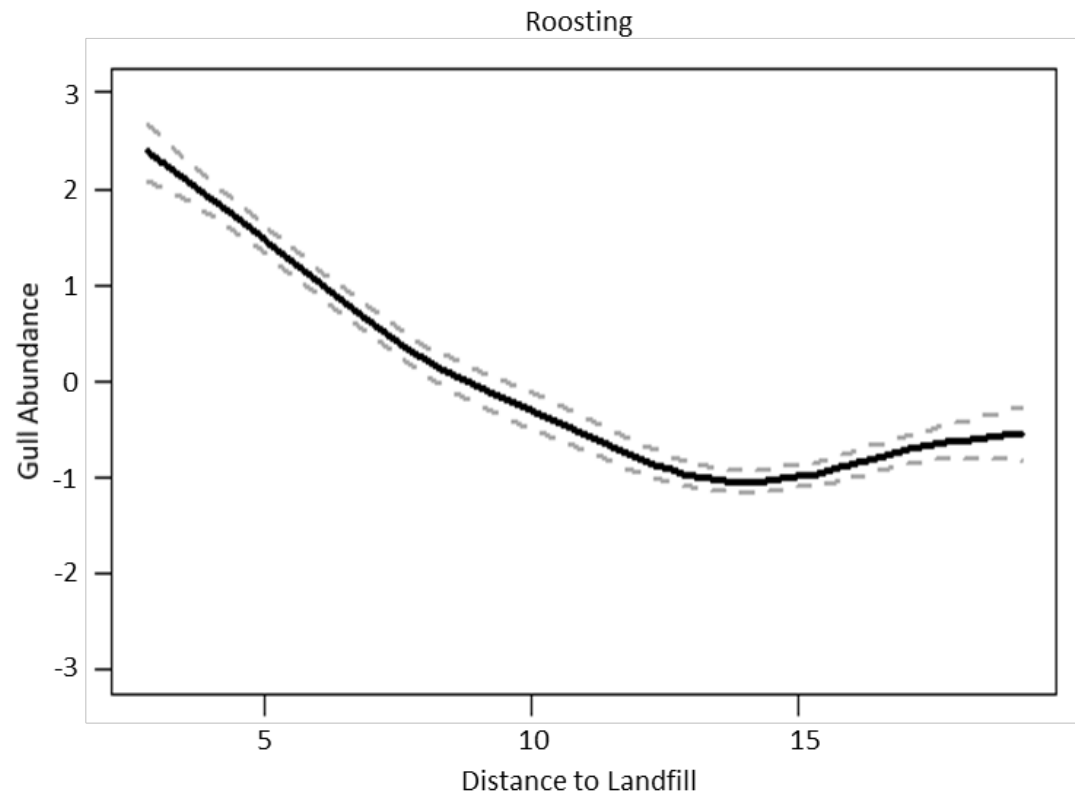

Figure 25. Gull abundance response to distance to landfill (in kilometers) from generalized additive mixed models at the pond scale in restoration ponds, South San Francisco Bay, California. Dashed lines indicate the 95percent confidence interval. 
A

Dabbling Duck

Diving Duck

Medium Shorebird

Small Shorebird

Gull

Piscivore

Tern

Wader

AMAV

EAGR

$\mathrm{NSHO}$

RUDU

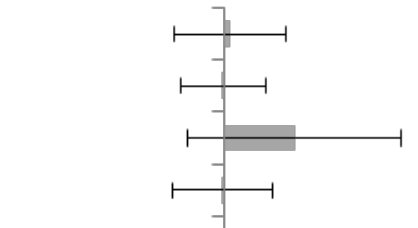

.

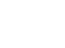

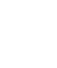

(15)

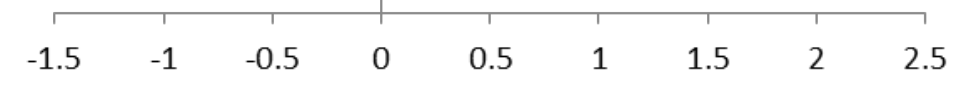

B

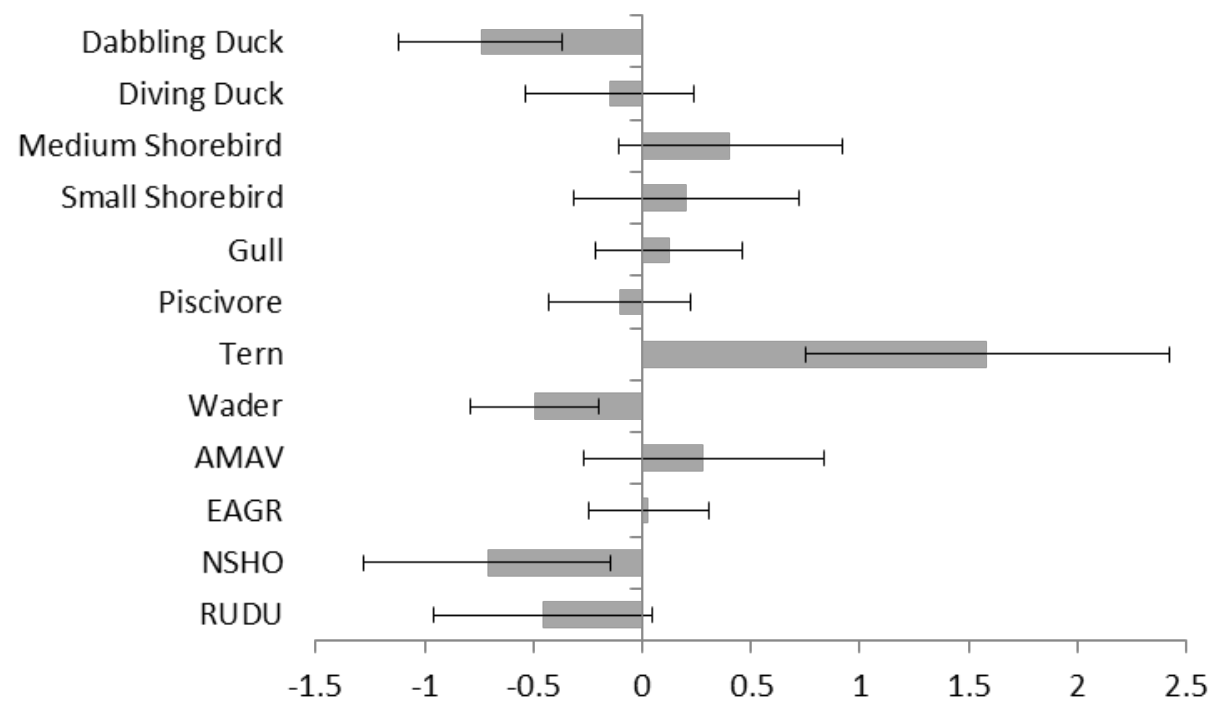

Figure 26. Model-averaged coefficients ( \pm 95 -percent confidence interval $[\mathrm{Cl}])$ of percent of pond levees open to hunting for (A) foraging and (B) roosting waterbirds at the pond scale in restoration ponds, South San Francisco Bay, California. Coefficients are considered significant only if the 95-percent $\mathrm{Cl}$ does not overlap zero. Bar length indicates the coefficient effect size and is comparable across species and guilds. AMAV, American avocet; EAGR, eared grebe; NSHO, northern shoveler; RUDU, ruddy duck. 
Foraging
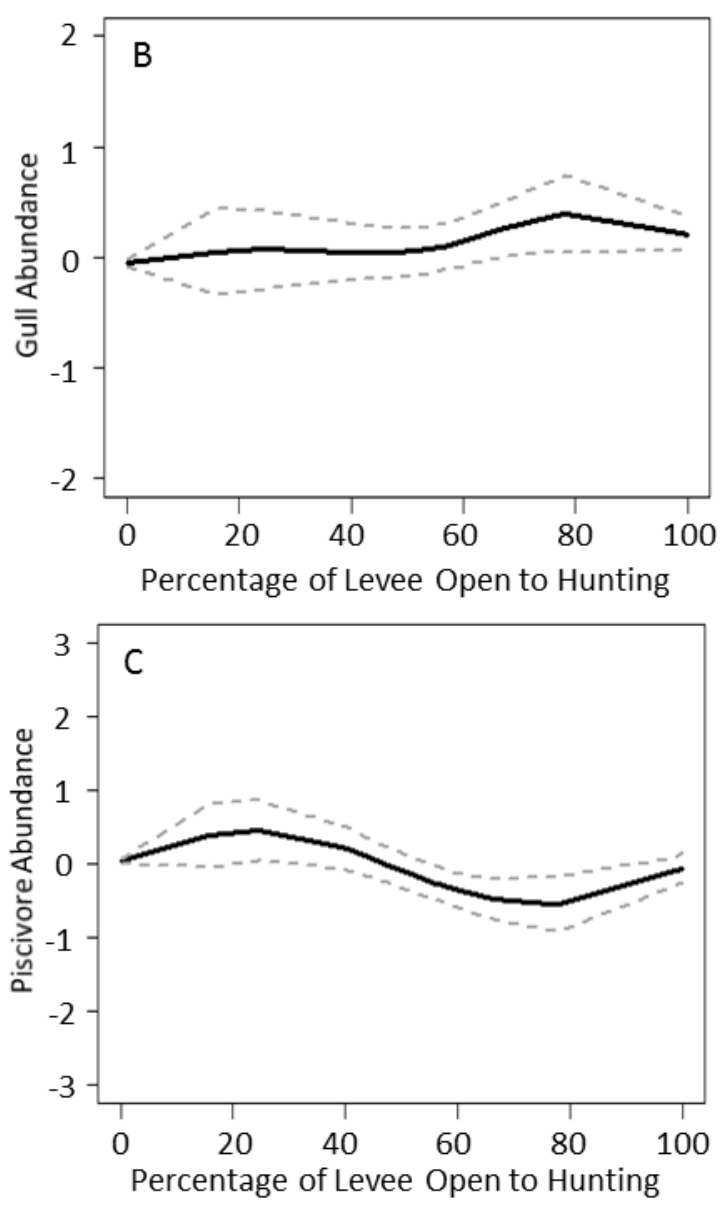

Roosting

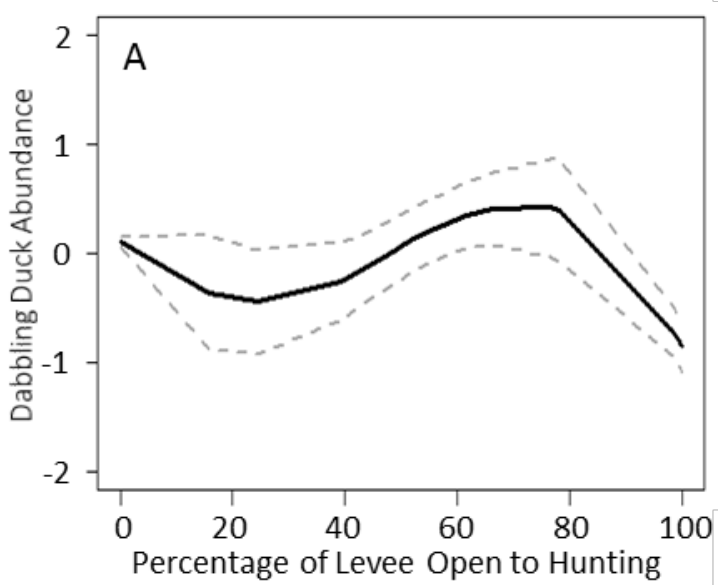

Percentage of Levee Open to Hunting

Figure 27. Dabbling duck (A), gull (B), piscivore (C), tern (D), and wader (E) abundance response to percentage of levees open to hunting from generalized additive mixed models at the pond scale in restoration ponds, South San Francisco Bay, California. Dashed lines indicate the 95-percent confidence interval. Note: Y-axis differs between figures. 

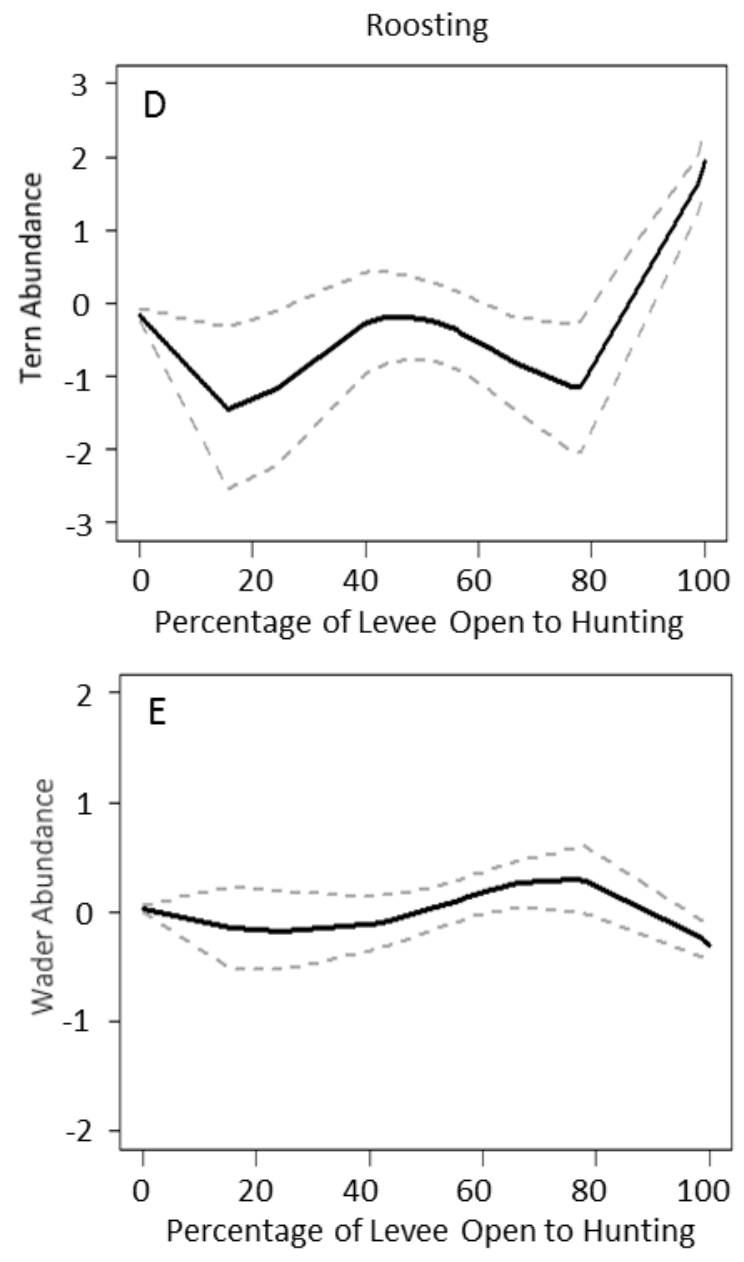

Figure 27.-Continued. 
A

Dabbling Duck

Diving Duck

Medium Shorebird

Small Shorebird

Gull

Piscivore

Tern

Wader

AMAV

EAGR

NSHO

RUDU

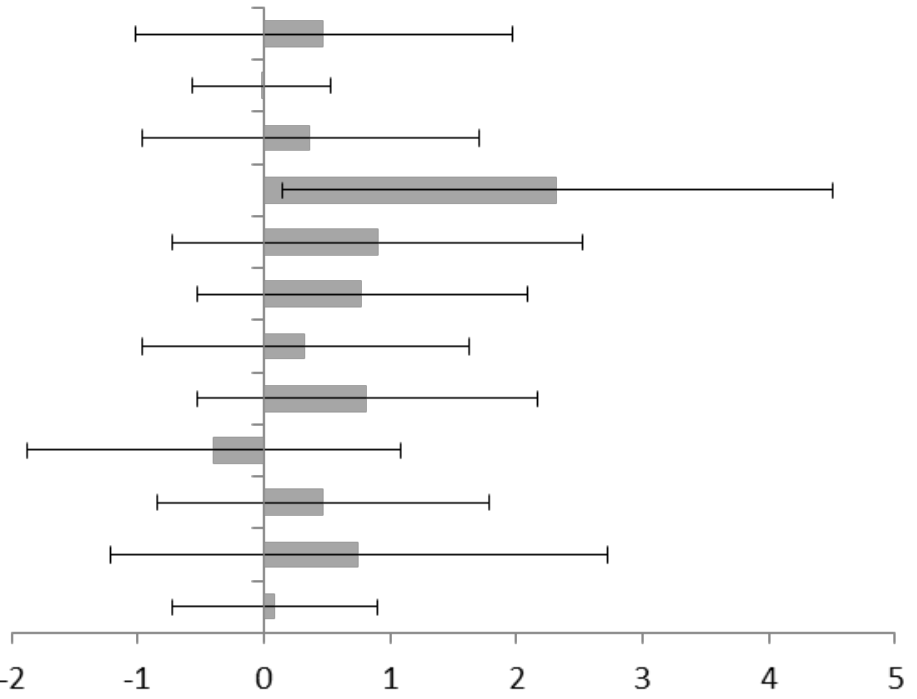

B

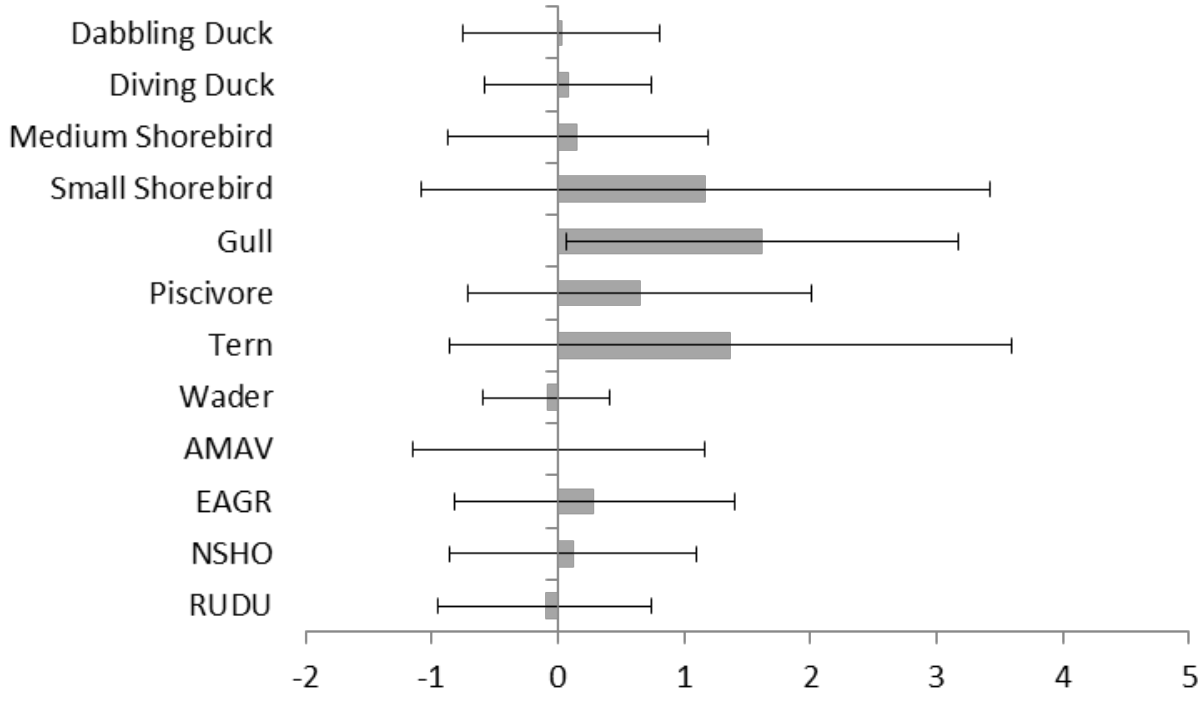

Figure 28. Model-averaged coefficients ( \pm 95 -percent confidence interval $[\mathrm{Cl}]$ ) of percentage of pond levees open to the public for $(\mathrm{A})$ foraging and $(\mathrm{B})$ roosting waterbirds at the pond scale in restoration ponds, South San Francisco Bay, California. Coefficients are considered significant only if the 95-percent Cl does not overlap zero. Bar length indicates the coefficient effect size and is comparable across species and guilds. AMAV, American avocet; EAGR, eared grebe; NSHO, northern shoveler; RUDU, ruddy duck. 

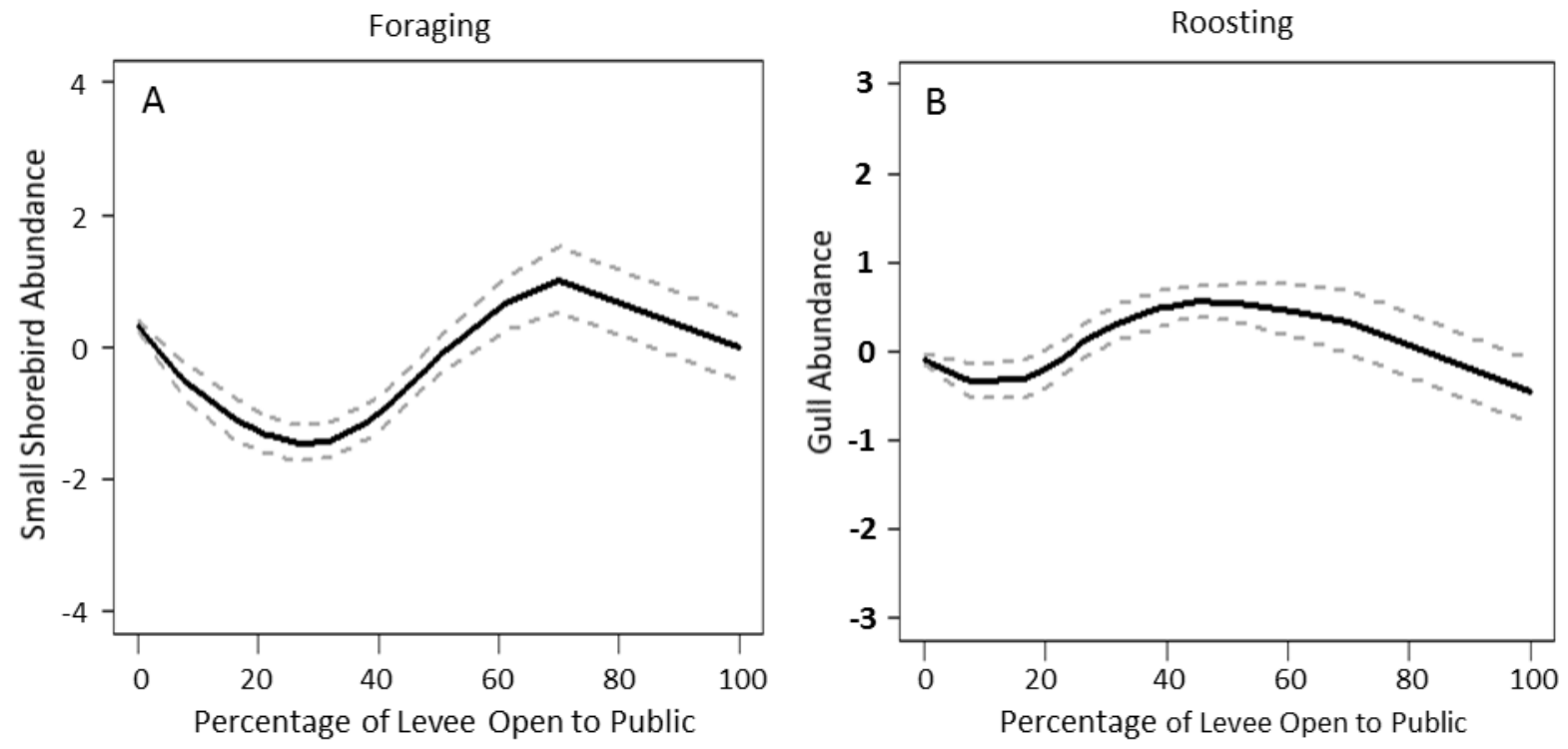

Figure 29. Small shorebird $(A)$ and gull $(B)$ abundance response to percentage of levees open to the public from generalized additive mixed models at the pond scale in restoration ponds, South San Francisco Bay, California. Dashed lines indicate the 95-percent confidence interval. Note: Y-axis differs between figures. 
A

Dabbling Duck

Diving Duck

Medium Shorebird

Small Shorebird

Gull

Piscivore

Tern

Wader

AMAV

EAGR

NSHO

RUDU

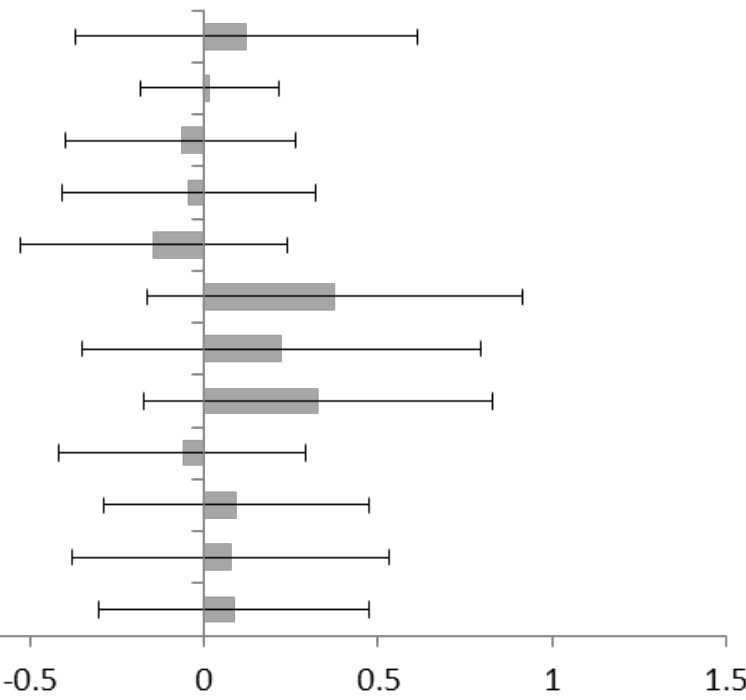

B

Dabbling Duck

Diving Duck

Medium Shorebird

Small Shorebird

Gull

Piscivore

Tern

Wader

AMAV

EAGR

$\mathrm{NSHO}$

RUDU

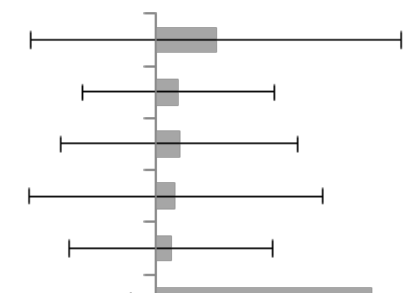

$-1 \quad-0.5$

.5


A

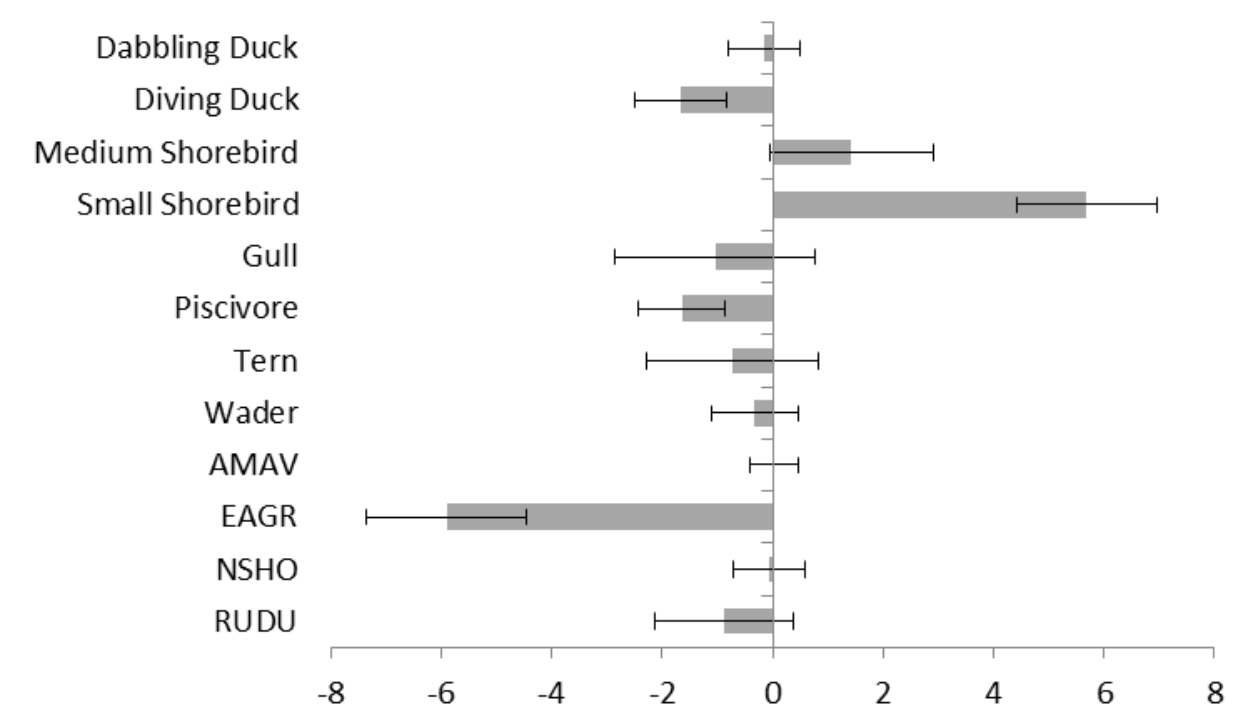

B

Dabbling Duck

Diving Duck

Medium Shorebird

Small Shorebird

Gull

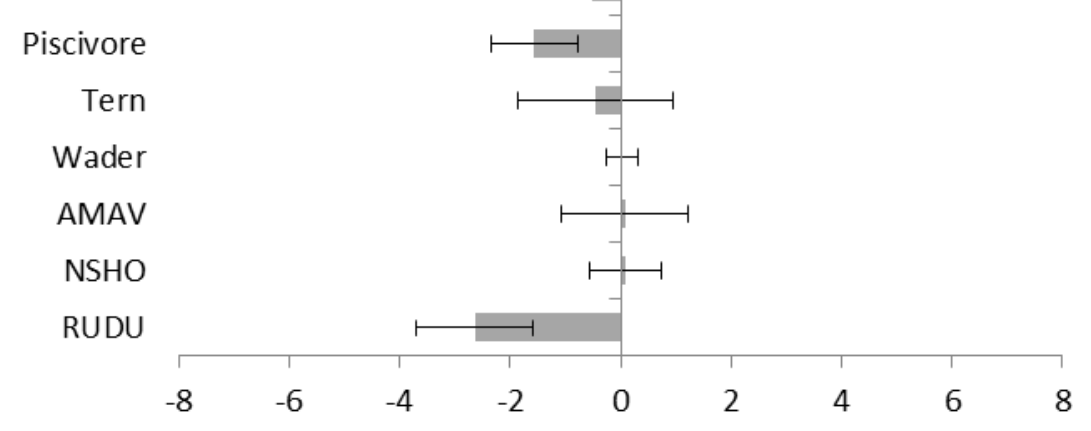

Figure 31. Model-averaged coefficients ( \pm 95 -percent confidence interval $[\mathrm{Cl}]$ ) of pond management (breached [positive values on $\mathrm{x}$-axis] compared to not breached [negative values on $\mathrm{x}$-axis]) for $(\mathrm{A})$ foraging and $(\mathrm{B})$ roosting waterbirds at the pond scale in restoration ponds, South San Francisco Bay, California. Coefficients are considered significant only if the 95-percent $\mathrm{Cl}$ does not overlap zero. Bar length indicates the coefficient effect size and is comparable across species and guilds. AMAV, American avocet; EAGR, eared grebe; NSHO, northern shoveler; RUDU, ruddy duck. The model-averaged parameter estimate (-26.998) for roosting eared grebe is not shown here as it exceeds the scale of this graph, but it is not considered significant (95-percent confidence interval overlaps zero). 

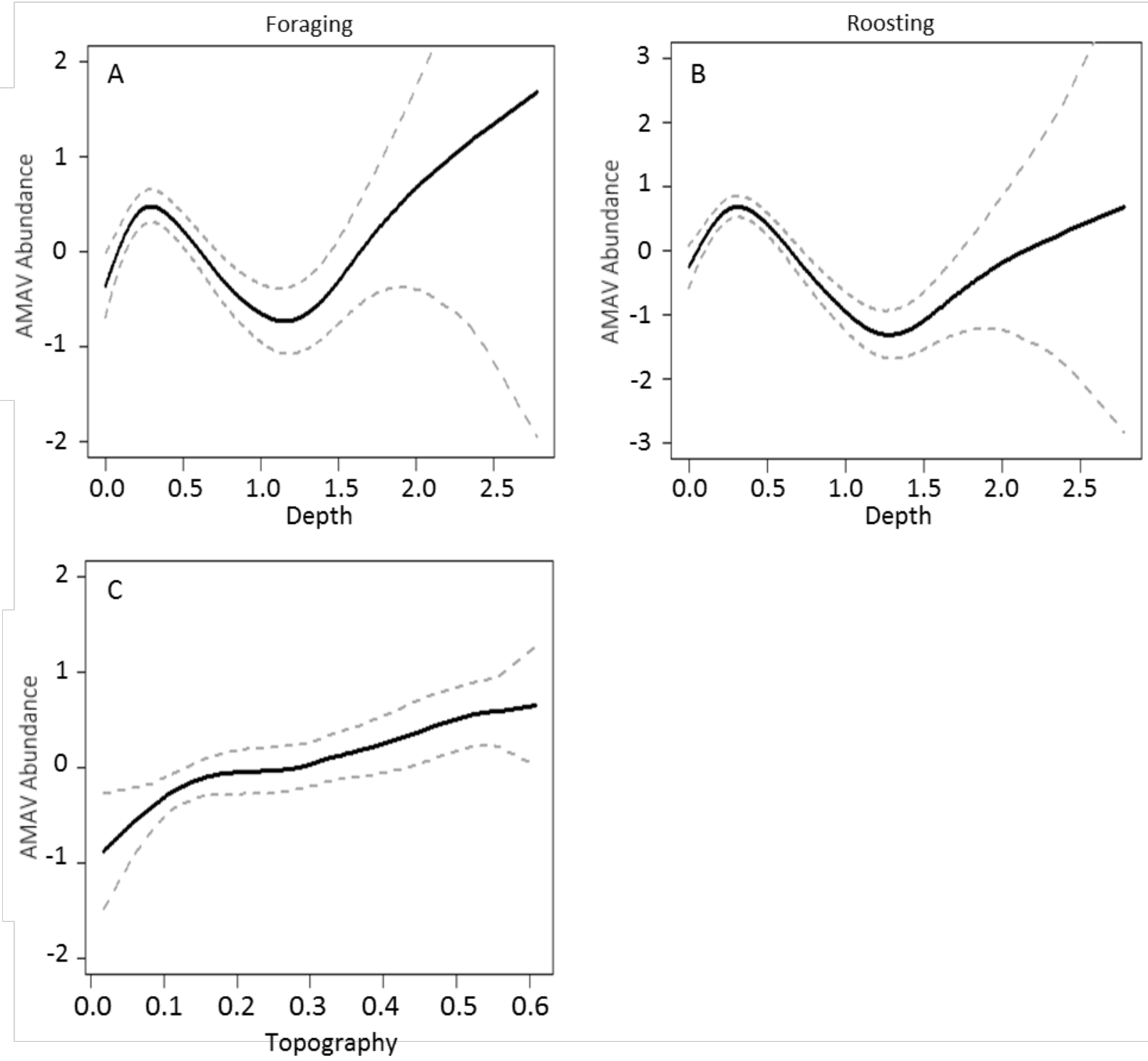

Figure 32. American avocet (AMAV) abundance response to depth (in meters relative to NAVD88 vertical datum; $A-B$ ) and topography (in meters; $C$ ) from generalized additive mixed models at the pond scale in restoration ponds, South San Francisco Bay, California. Dashed lines indicate the 95-percent confidence interval. 

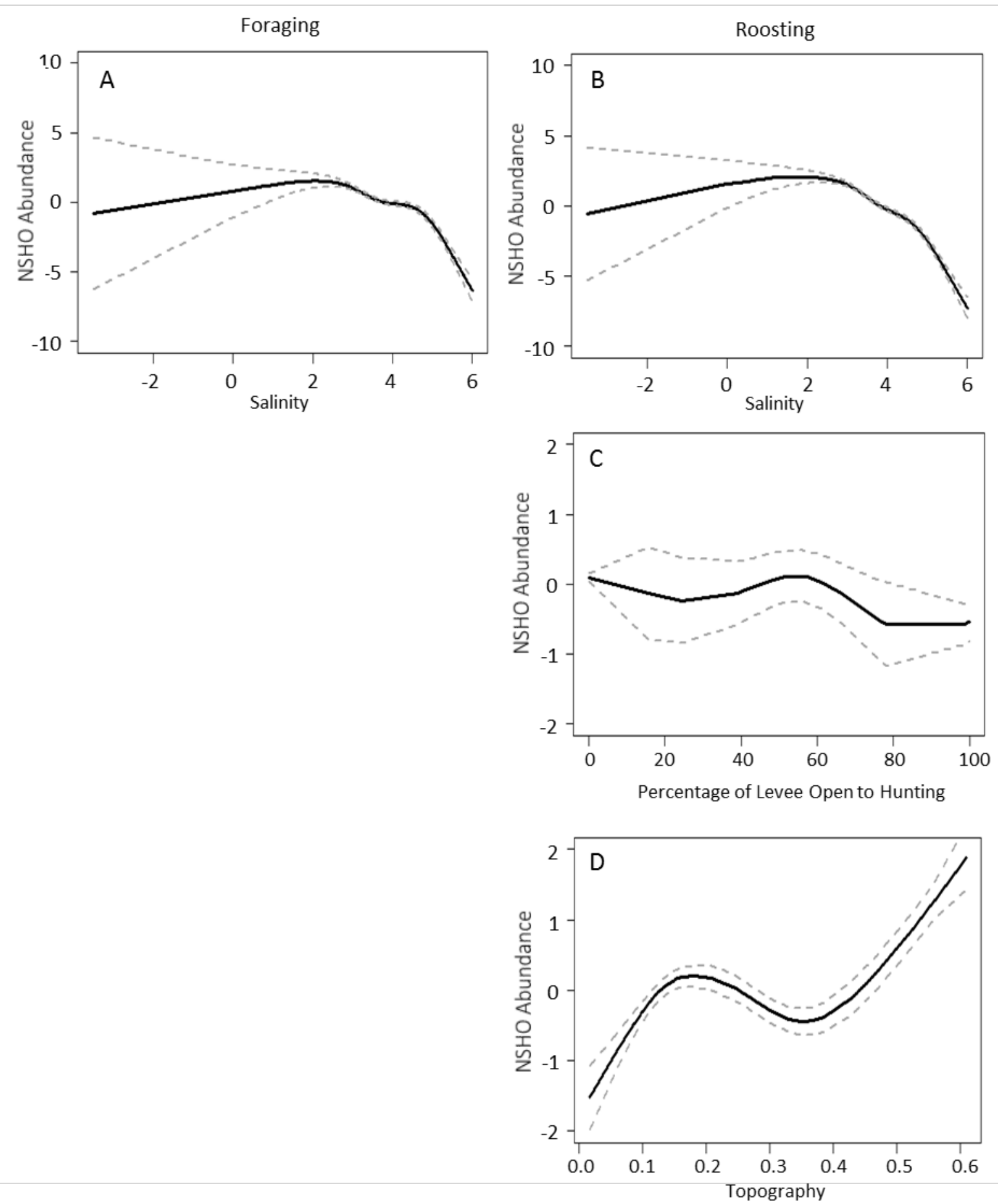

Figure 33. Northern shoveler (NSHO) abundance response to salinity (in log[parts per thousand]; A-B), percentage of the levee open to hunting $(C)$, and pond topography (in meters; $D$ ) from generalized additive mixed models at the pond scale in restoration ponds, South San Francisco Bay, California. Dashed lines indicate the 95percent confidence interval. 

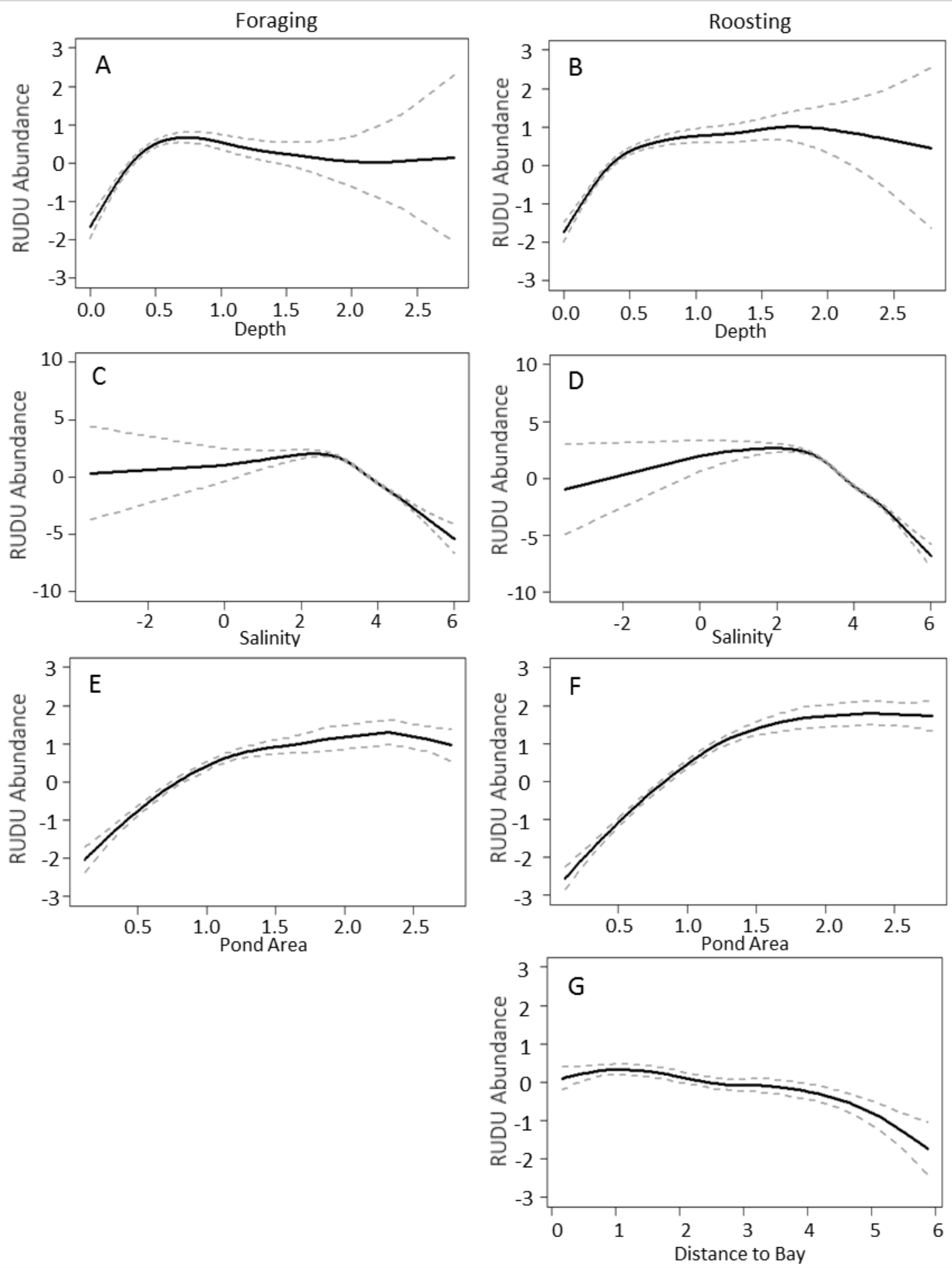

Figure 34. Ruddy duck (RUDU) abundance response to water depth (in meters; A-B), salinity (in log[parts per thousand]; C-D), pond area (in square kilometers; E-F), and distance to San Francisco Bay (in kilometers; G) from generalized additive mixed models at the pond scale in restoration ponds, South San Francisco Bay, California. Dashed lines indicate the 95-percent confidence interval. 

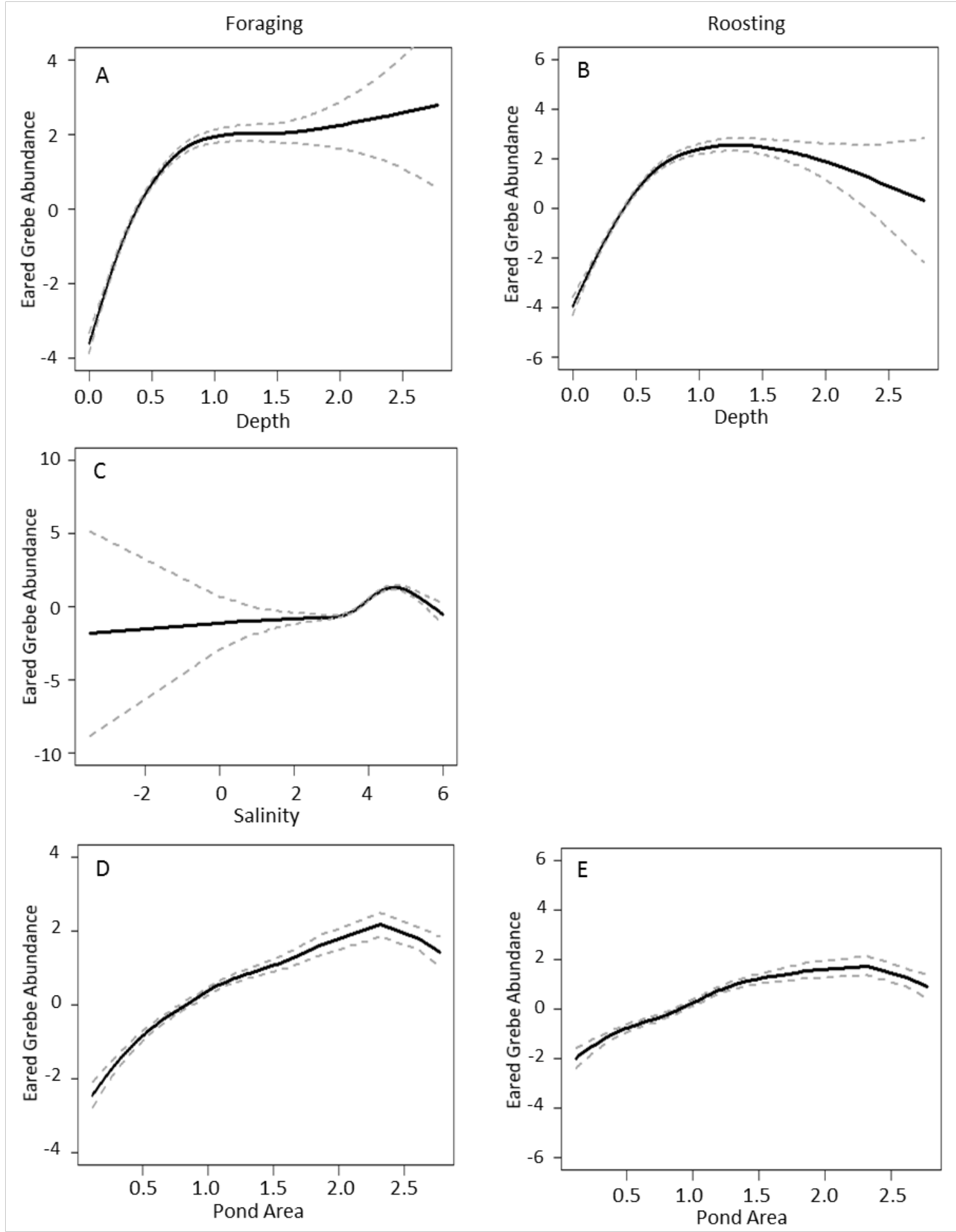

Figure 35. Eared grebe (EAGR) abundance response to depth (in meters relative to NAVD 88 vertical datum; AB), salinity (in log[parts per thousand]; C), and pond area (in square kilometers; D-E) from generalized additive mixed models at the pond scale in restoration ponds, South San Francisco Bay, California. Dashed lines indicate the 95-percent confidence interval. Note: Y-axis may differ among figures. 


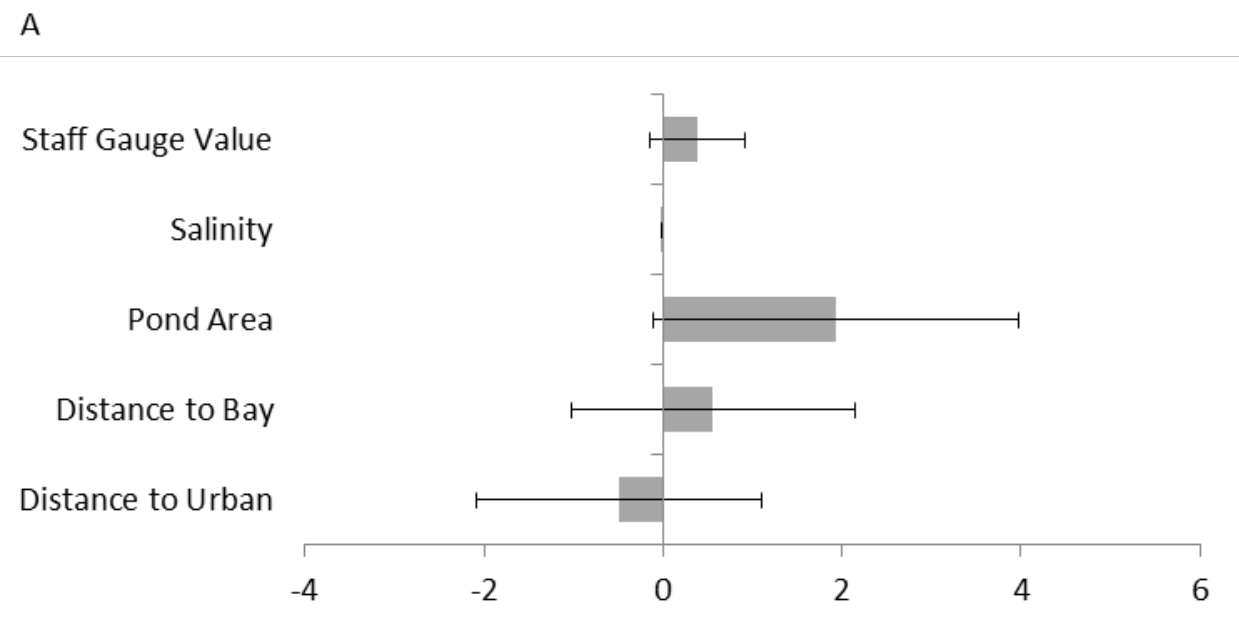

B

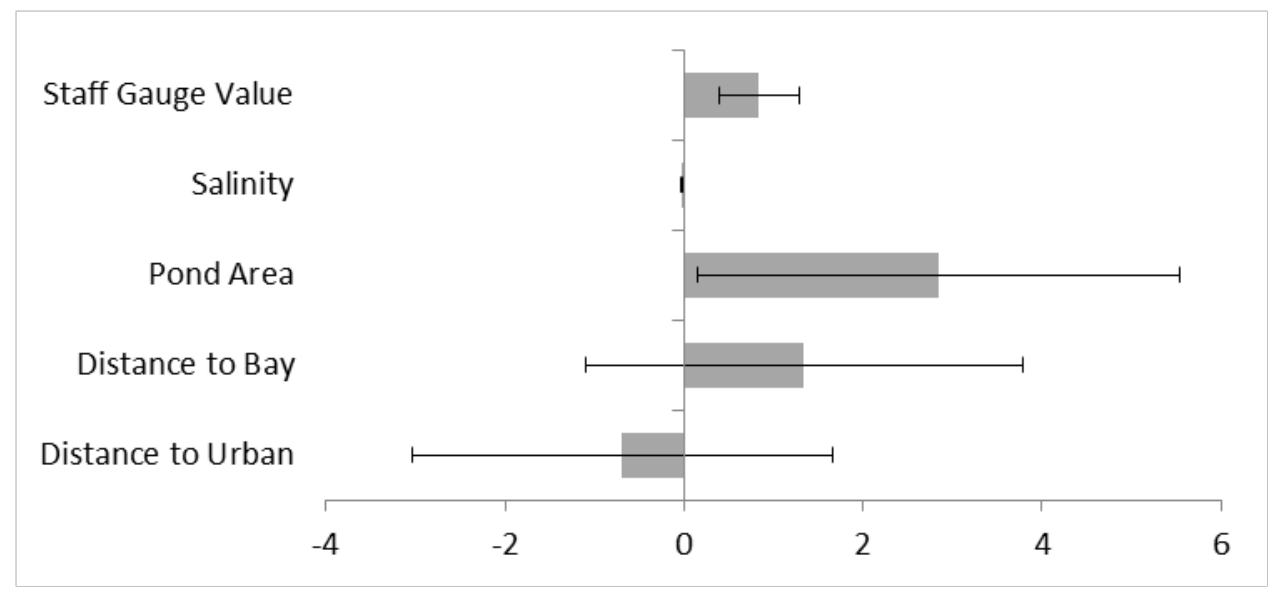

Figure 36. Model-averaged coefficients ( \pm 95 -percent confidence interval $[\mathrm{Cl}])$ for $(\mathrm{A})$ foraging and $(\mathrm{B})$ roosting eared grebes in salt production ponds at the pond scale in restoration ponds, South San Francisco Bay, California. Coefficients are considered significant only if the 95-percent $\mathrm{Cl}$ does not overlap zero. Bar length indicates the coefficient effect size and is comparable across species and guilds. 
A

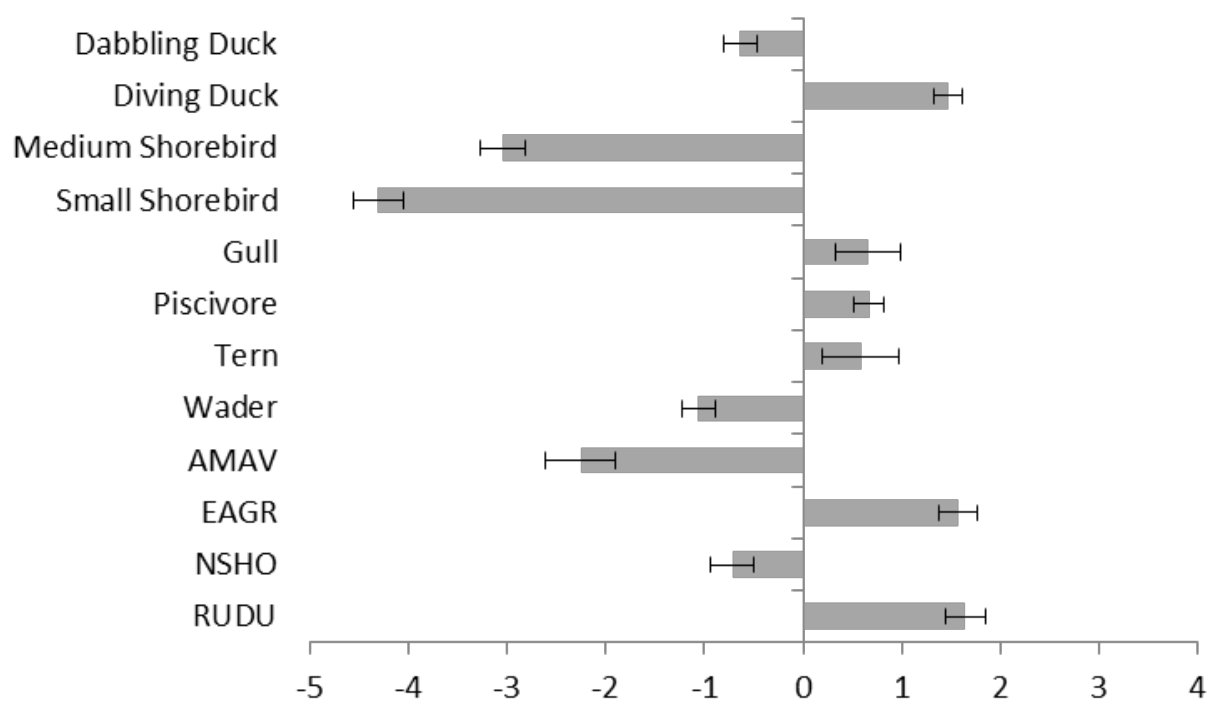

B

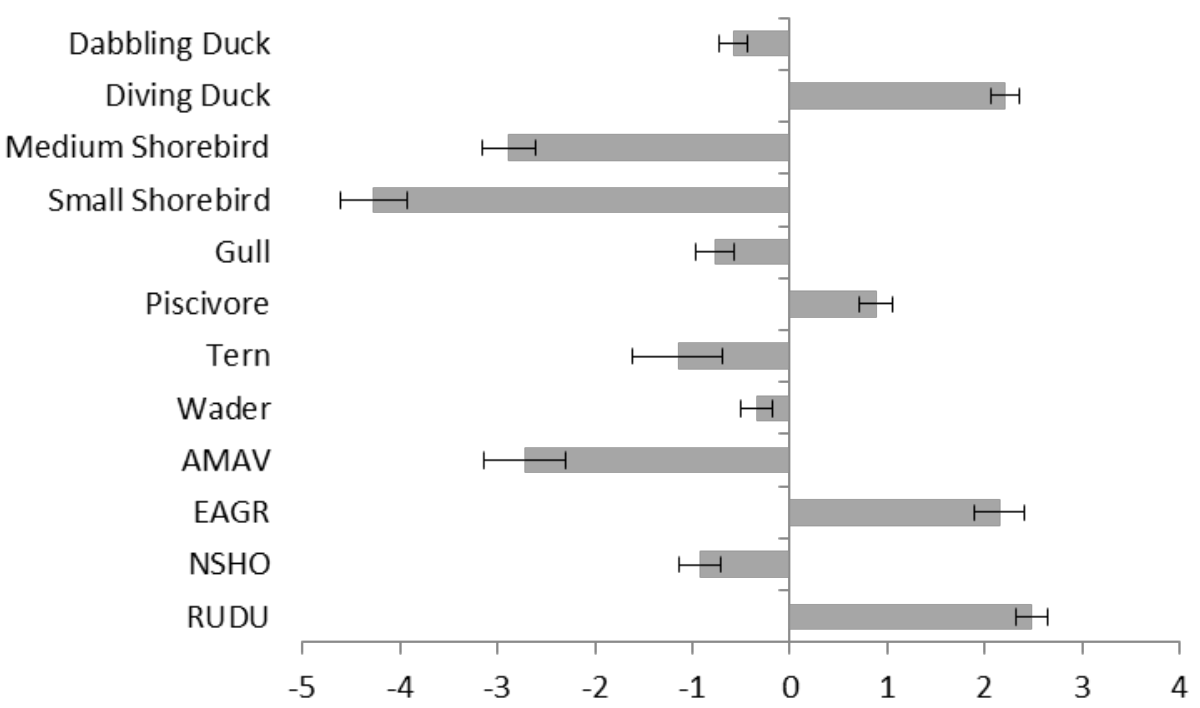

Figure 37. Model-averaged coefficients ( \pm 95 -percent confidence interval $[\mathrm{Cl}])$ of grid water depth for $(\mathrm{A})$ foraging and $(B)$ roosting waterbirds at the grid scale in restoration ponds, South San Francisco Bay, California.

Coefficients are considered significant only if the 95-percent $\mathrm{Cl}$ does not overlap zero. Bar length indicates the coefficient effect size and is comparable across species and guilds. AMAV, American avocet; EAGR, eared grebe; NSHO, northern shoveler; RUDU, ruddy duck. 

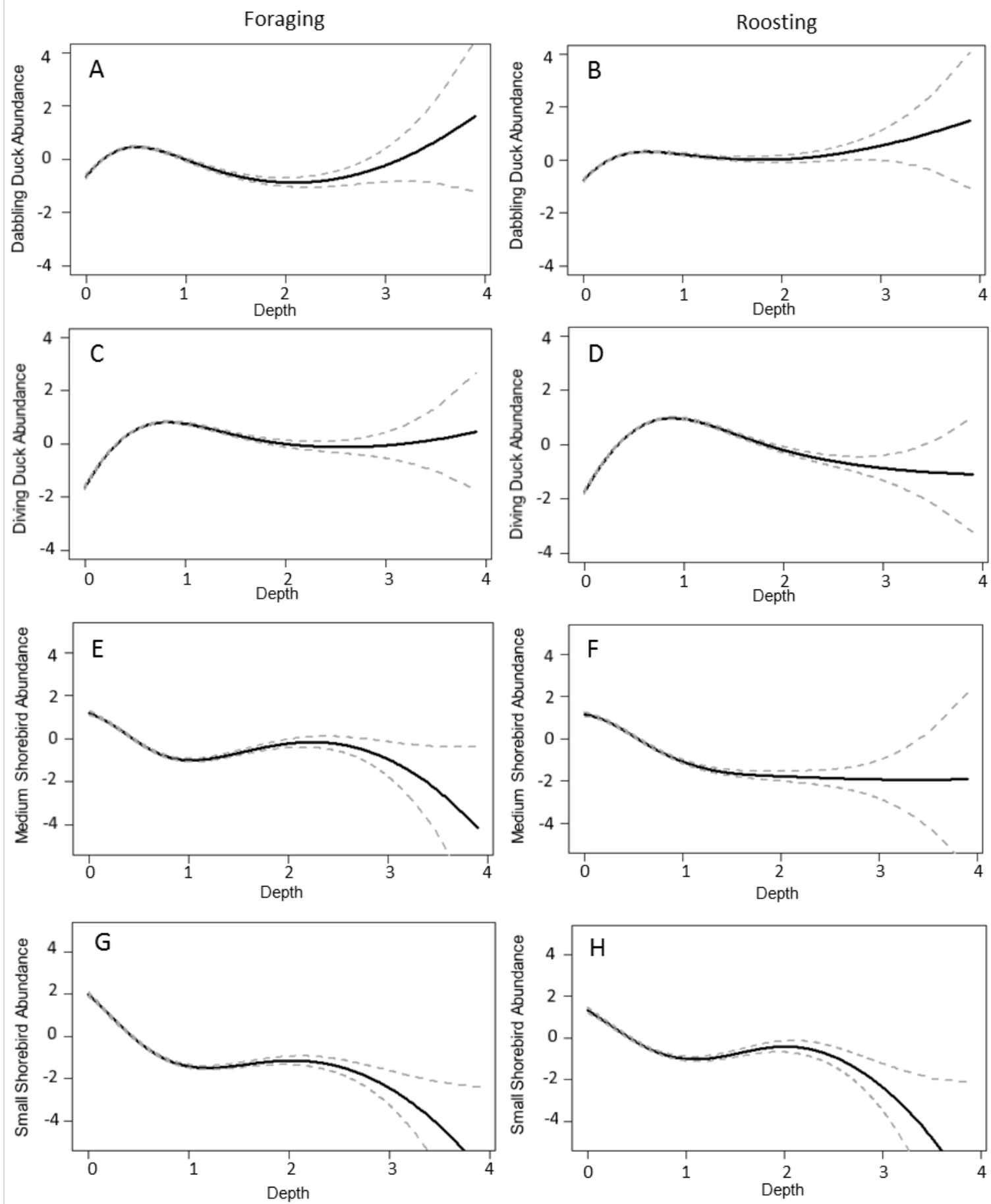

Figure 38. Dabbling duck (A-B), diving duck (C-D), medium $(E-F)$ and small shorebird $(G-H)$, gull $(I-J)$, piscivore $(K-L)$, tern $(M-N)$, and wader $(O-P)$ abundance response to water depth (in meters relative to NAVD 88 vertical datum) from generalized additive models at the grid scale in restoration ponds, South San Francisco Bay, California. Dashed lines indicate 95-percent confidence interval. 

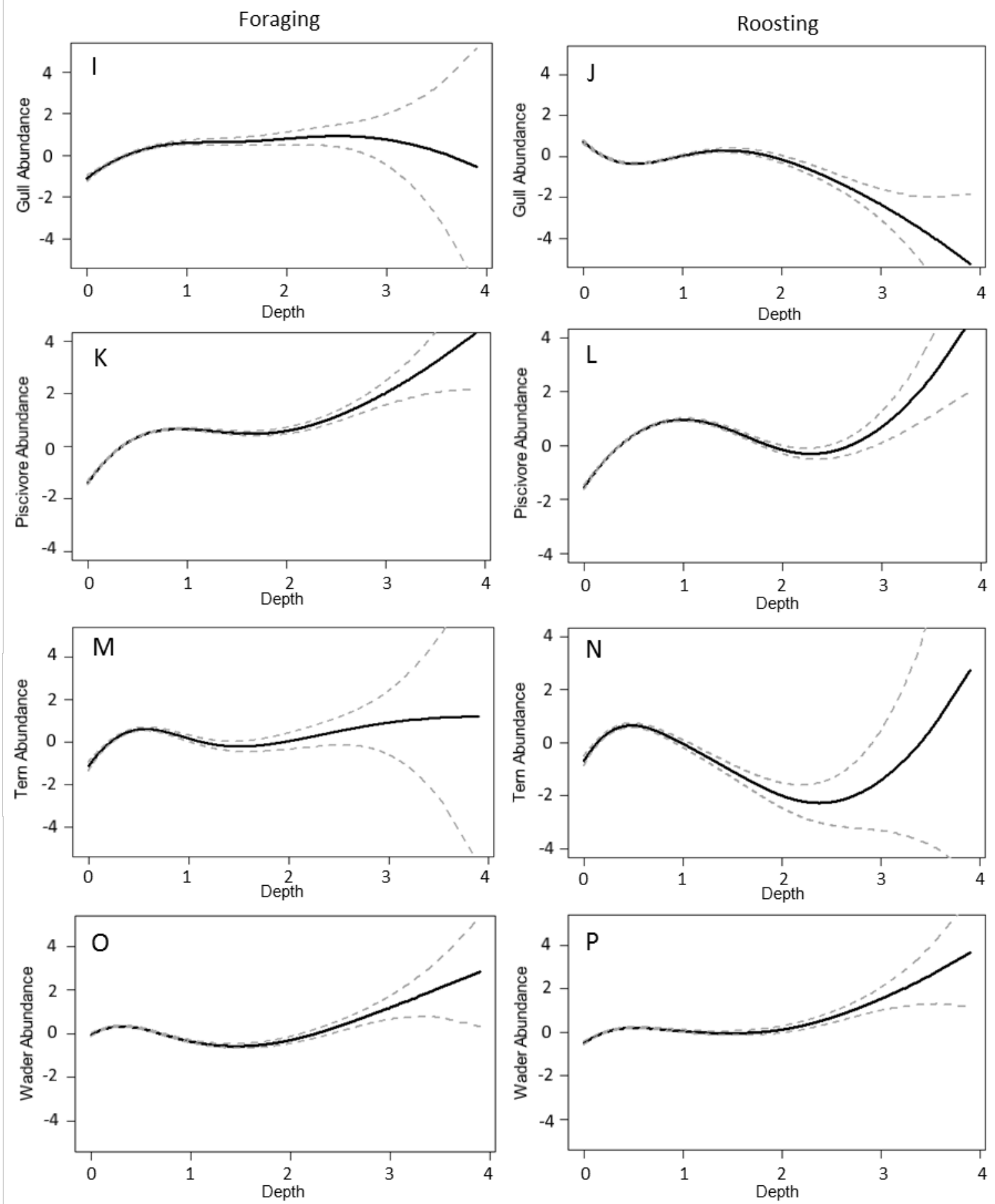

Figure 38. - Continued. 
A

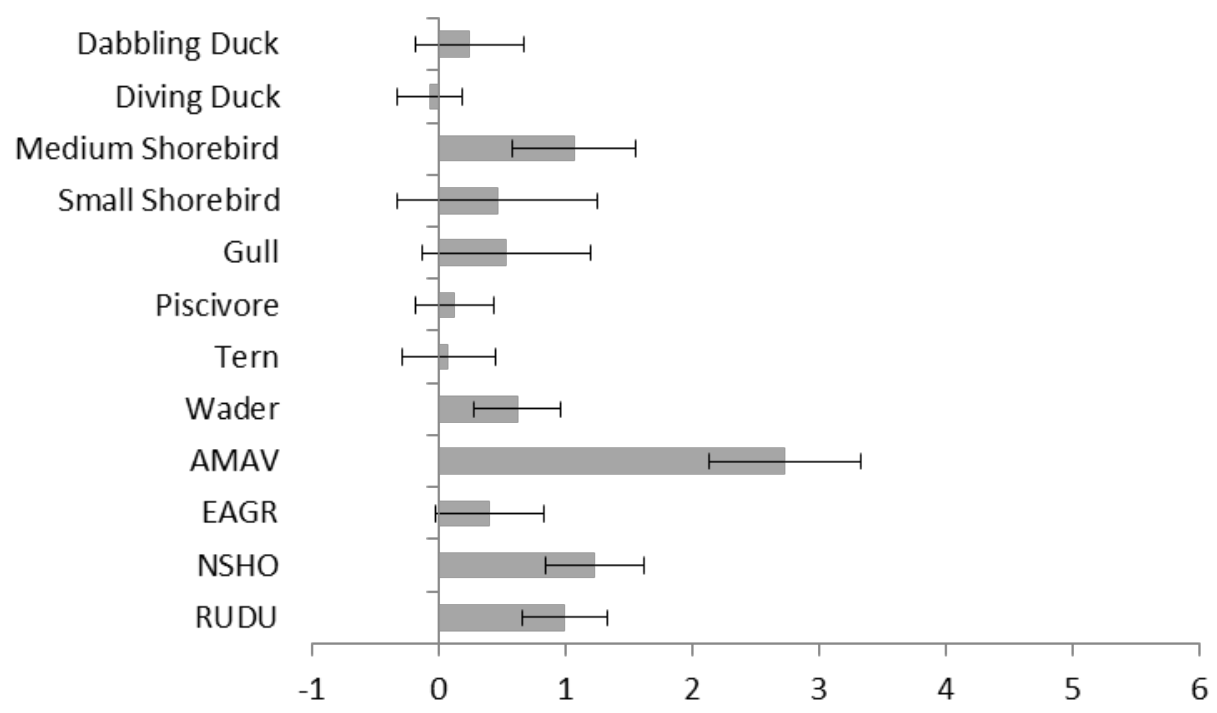

B

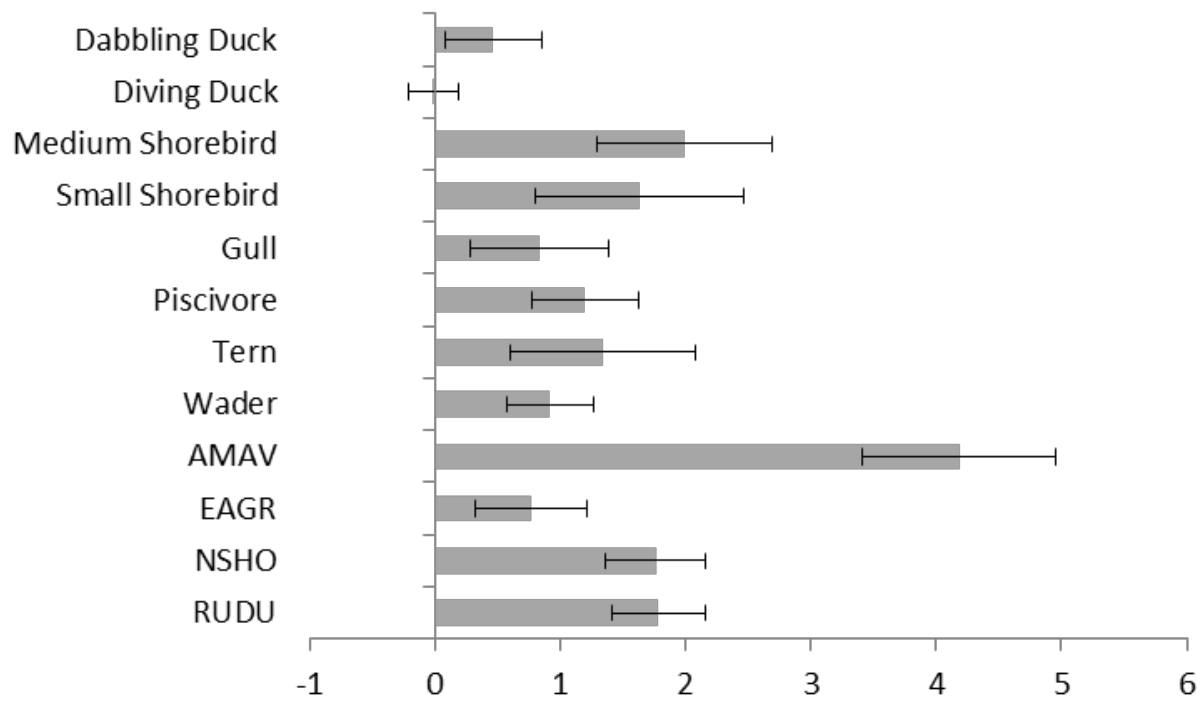

Figure 39. Model-averaged coefficients ( \pm 95 -percent confidence interval $[\mathrm{Cl}]$ ) of islands for $(\mathrm{A})$ foraging and $(\mathrm{B})$ roosting waterbirds at the grid scale in restoration ponds, South San Francisco Bay, California. Coefficients are considered significant only if the 95-percent $\mathrm{Cl}$ does not overlap zero. Bar length indicates the coefficient effect size and is comparable across species and guilds. AMAV, American avocet; EAGR, eared grebe; NSHO, northern shoveler; RUDU, ruddy duck. 


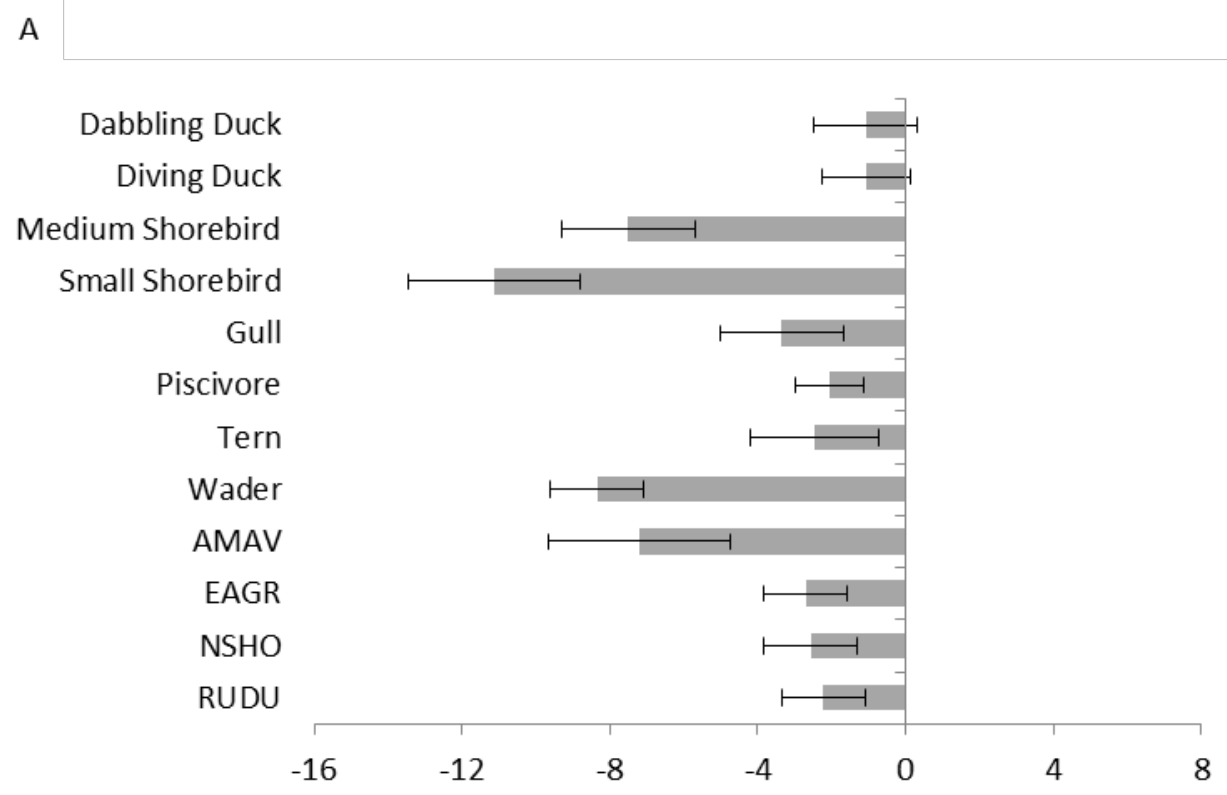

B

Dabbling Duck Diving Duck Medium Shorebird Small Shorebird

Gull Piscivore

Tern

Wader

AMAV

EAGR

$\mathrm{NSHO}$

RUDU
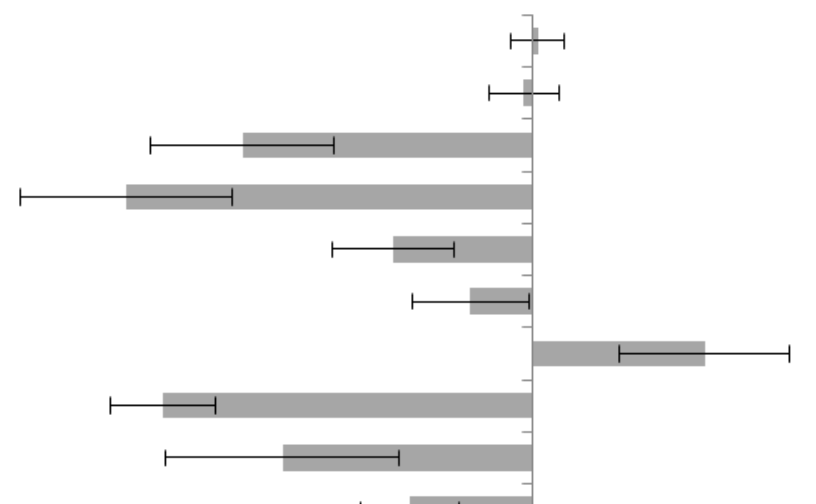

$-16$

$-12$

Figure 40. Model-averaged coefficients ( \pm 95 -percent confidence interval $[\mathrm{Cl}]$ ) of grid distance to levee for (A) foraging and (B) roosting waterbirds at the grid scale in restoration ponds, South San Francisco Bay, California. Coefficients are considered significant only if the 95 -percent $\mathrm{Cl}$ does not overlap zero. Bar length indicates the coefficient effect size and is comparable across species and guilds. AMAV, American avocet; EAGR, eared grebe; NSHO, northern shoveler; RUDU, ruddy duck. 

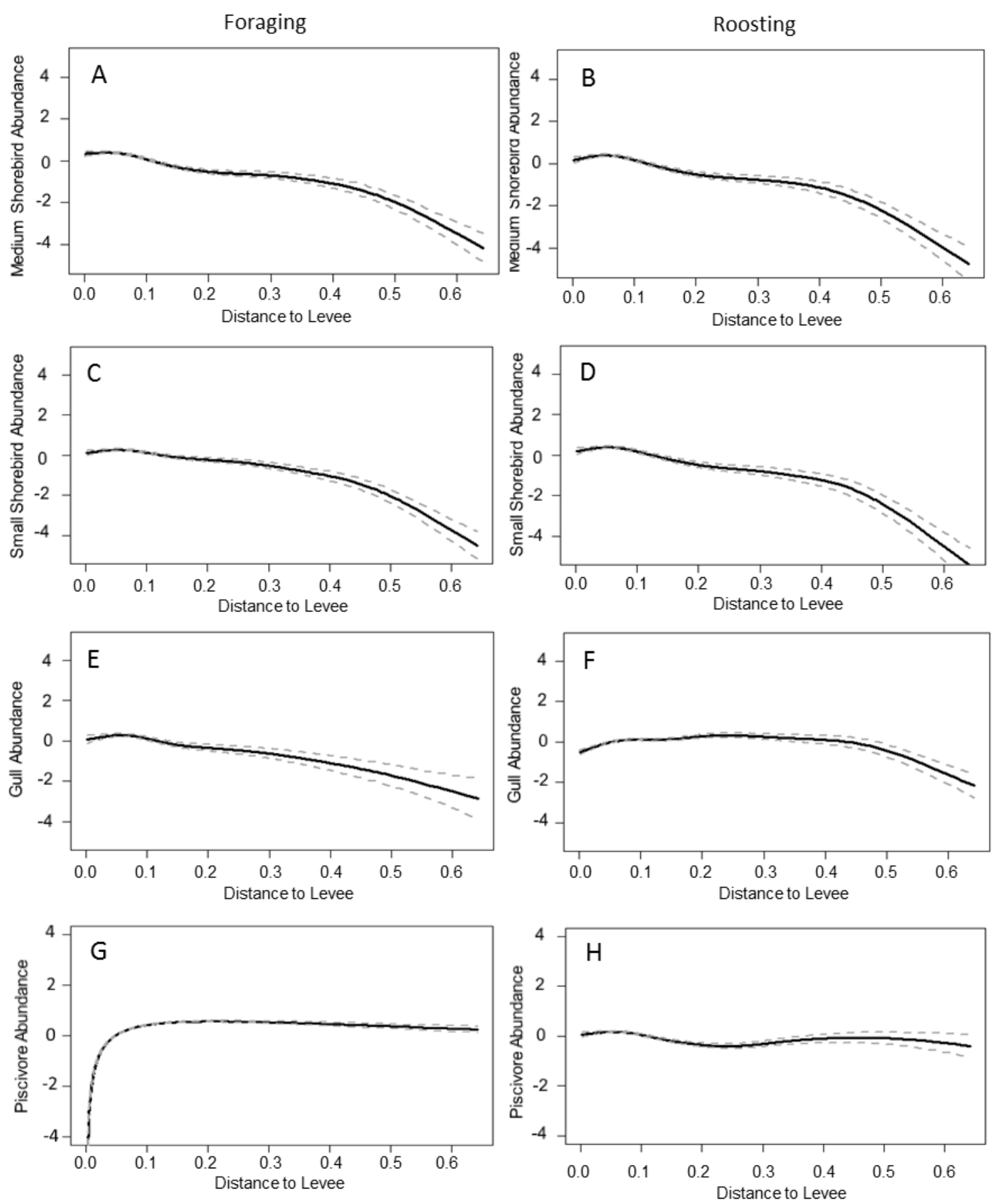

Figure 41. Medium (A-B) and small shorebird $(C-D)$, gull $(E-F)$, piscivore $(G-H)$, tern $(I-J)$, and wader $(K-L)$ abundance response to grid distance to levees (in kilometers) from generalized additive models at the grid scale in restoration ponds, South San Francisco Bay, California. Dashed lines indicate 95-percent confidence interval. 

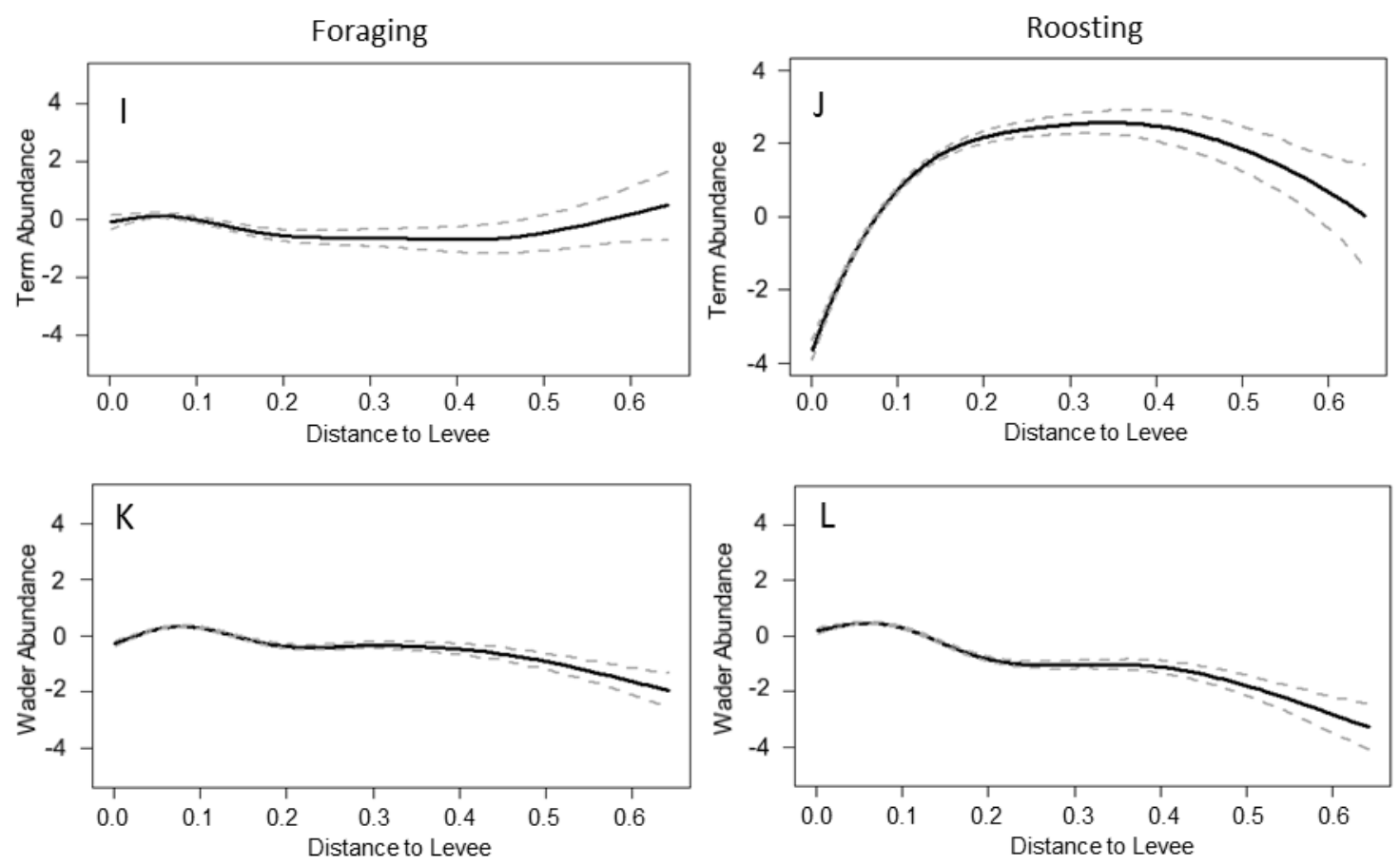

Figure 41. - Continued. 


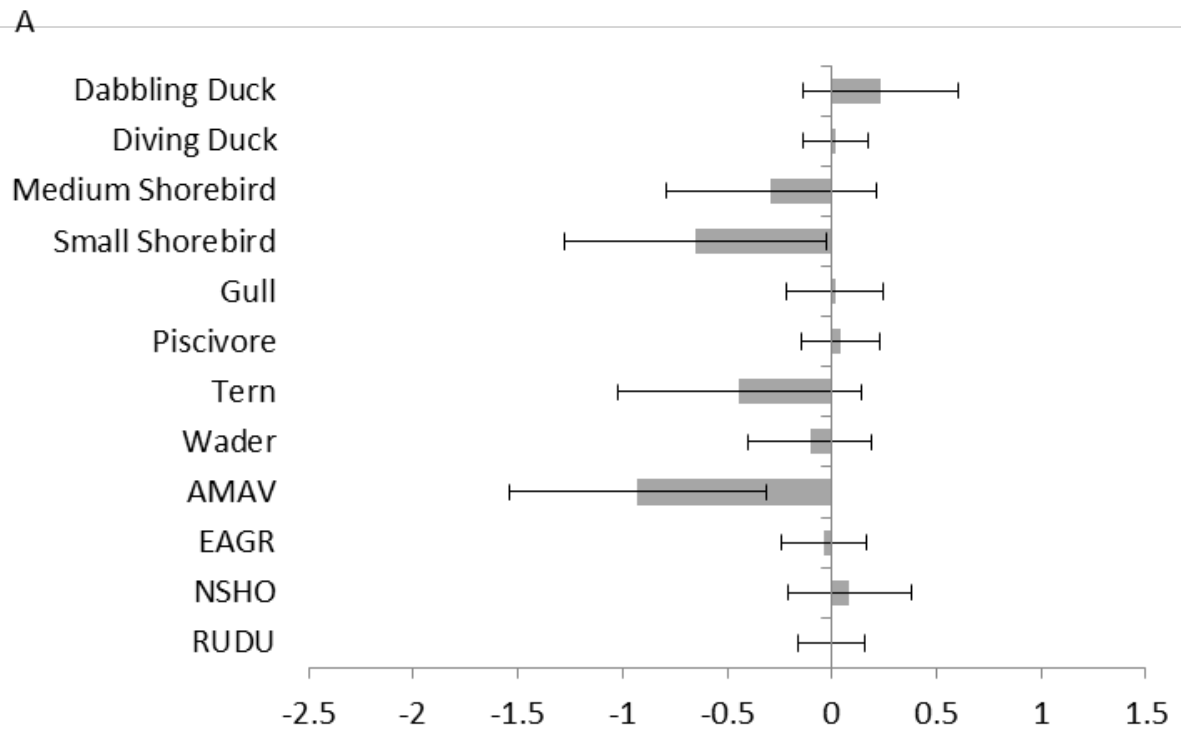

B

Dabbling Duck Diving Duck Medium Shorebird Small Shorebird

Gull Piscivore

Tern

Wader

AMAV

EAGR

$\mathrm{NSHO}$

RUDU

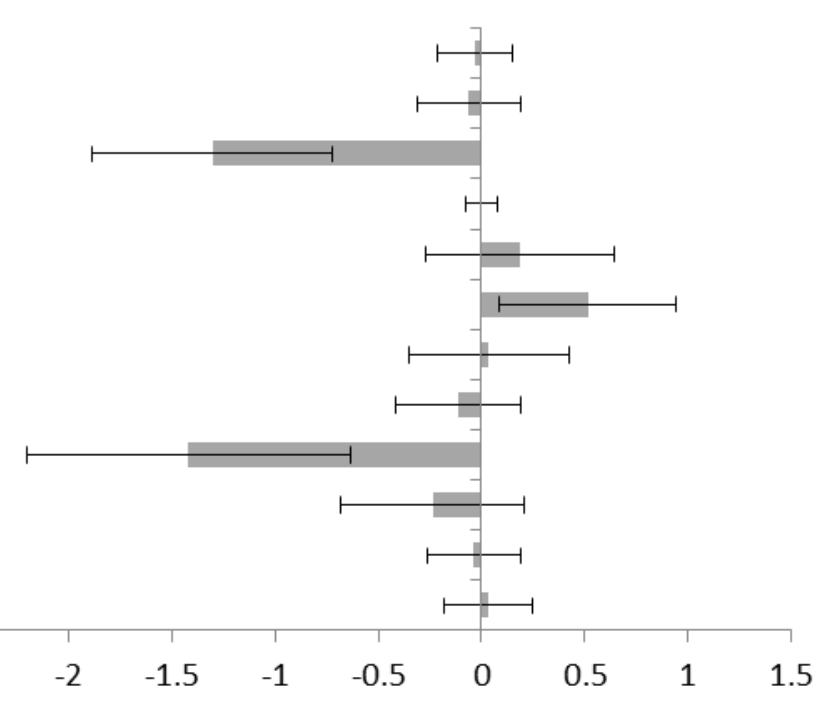

Figure 42. Model-averaged coefficients ( \pm 95 -percent confidence interval $[\mathrm{Cl}]$ ) of grid distance to creek for (A) foraging and (B) roosting waterbirds at the grid scale in restoration ponds, South San Francisco Bay, California. Coefficients are considered significant only if the 95-percent $\mathrm{Cl}$ does not overlap zero. Bar length indicates the coefficient effect size and is comparable across species and guilds. AMAV, American avocet; EAGR, eared grebe; NSHO, northern shoveler; RUDU, ruddy duck. 

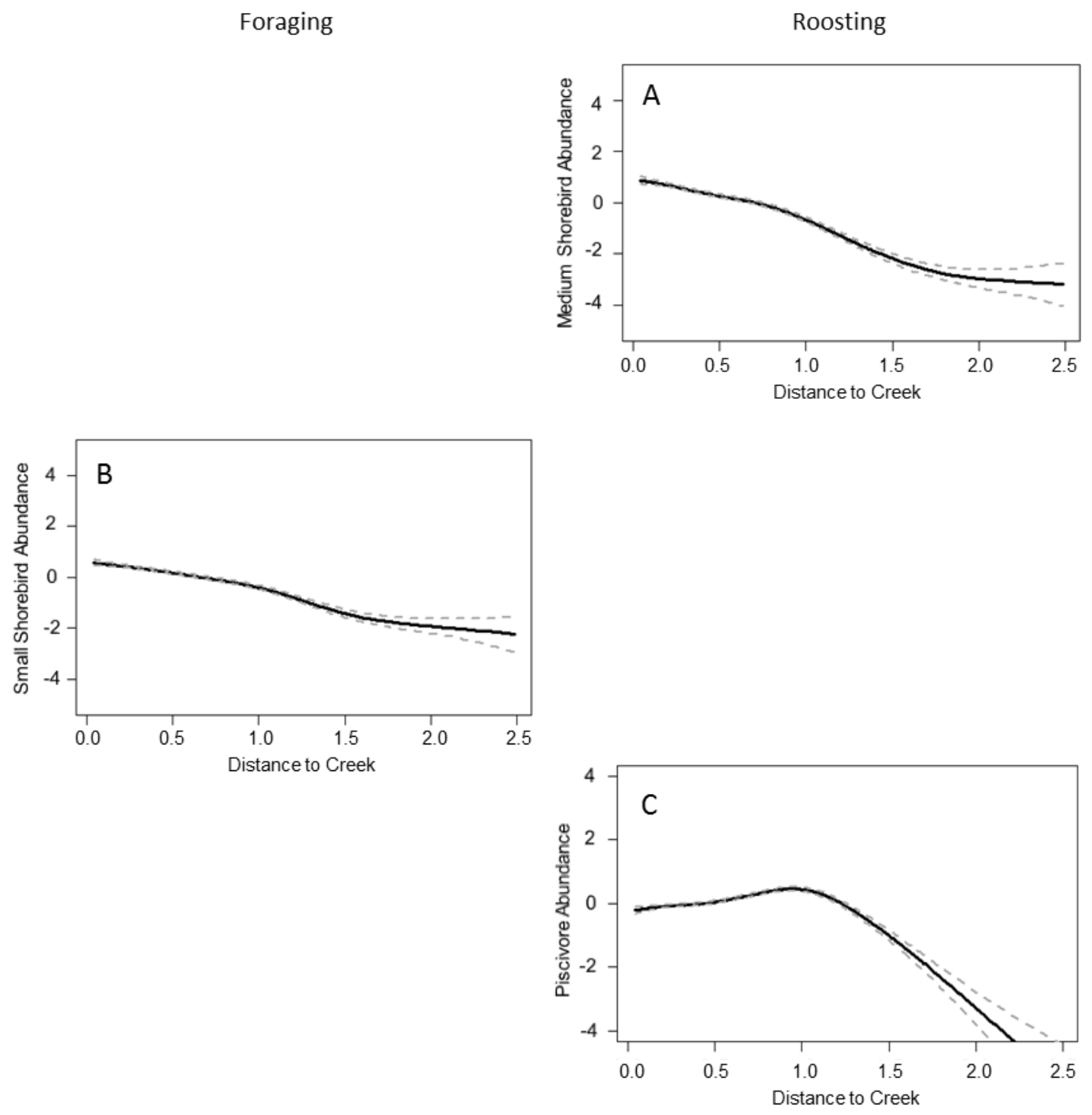

Figure 43. Medium shorebird (A), small shorebird (B) and piscivore (C) abundance response to grid distance to adjacent creek (in kilometers) from generalized additive models at the grid scale in restoration ponds, South San Francisco Bay, California. Dashed lines indicate 95-percent confidence interval. 

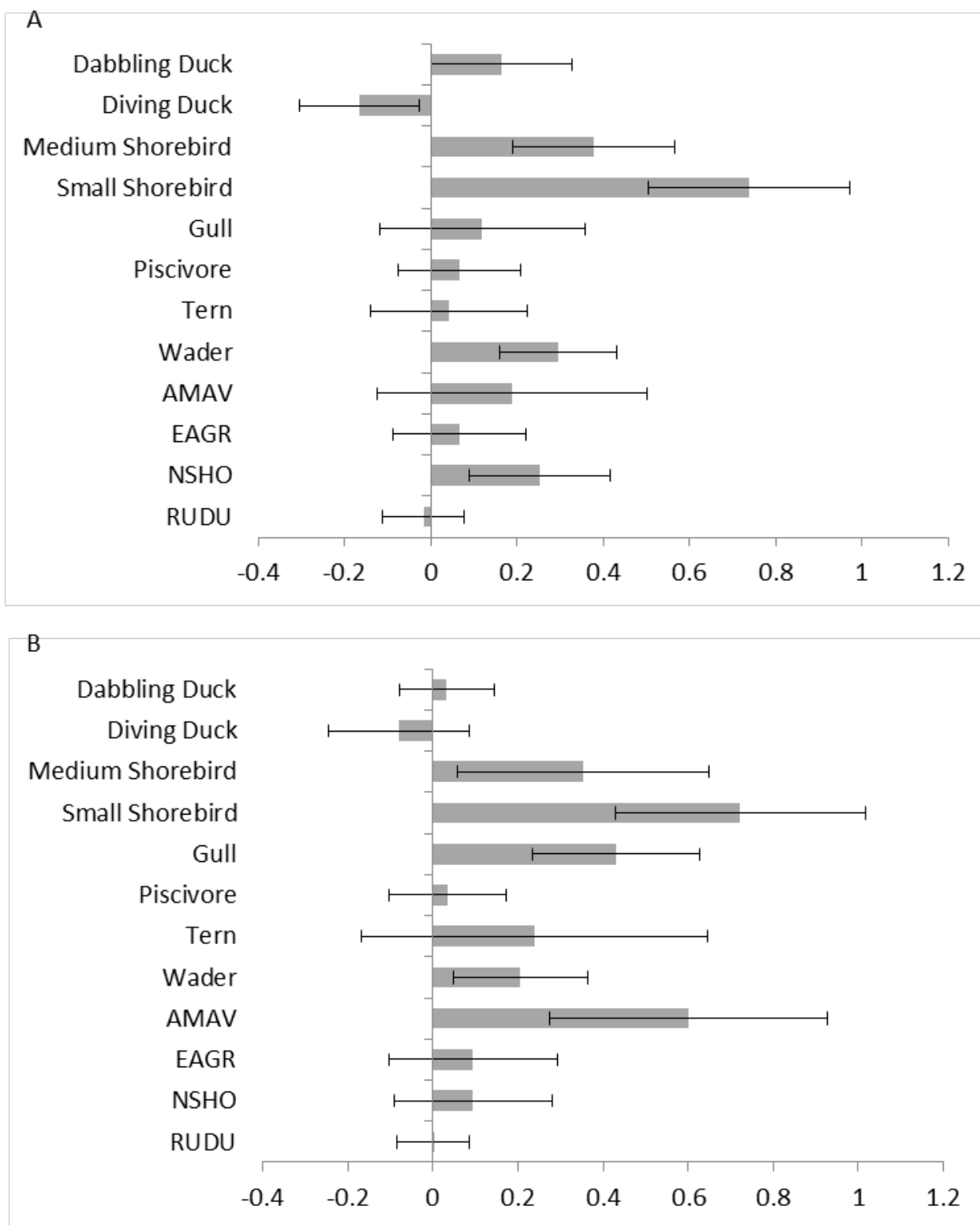

Figure 44. Model-averaged coefficients ( \pm 95 -percent confidence interval $[\mathrm{Cl}])$ of grid topography for $(\mathrm{A})$ foraging and (B) roosting waterbirds at the grid scale in restoration ponds, South San Francisco Bay, California. Coefficients are considered significant only if the 95-percent $\mathrm{Cl}$ does not overlap zero. Bar length indicates the coefficient effect size and is comparable across species and guilds. AMAV, American avocet; EAGR, eared grebe; NSHO, northern shoveler; RUDU, ruddy duck. 


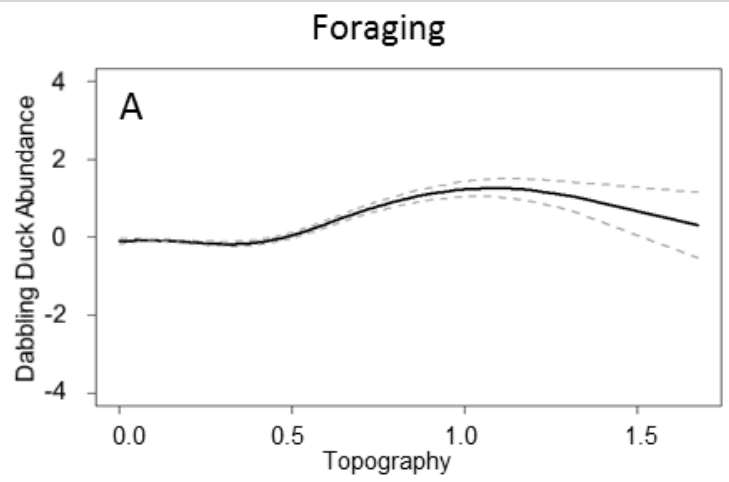

Roosting
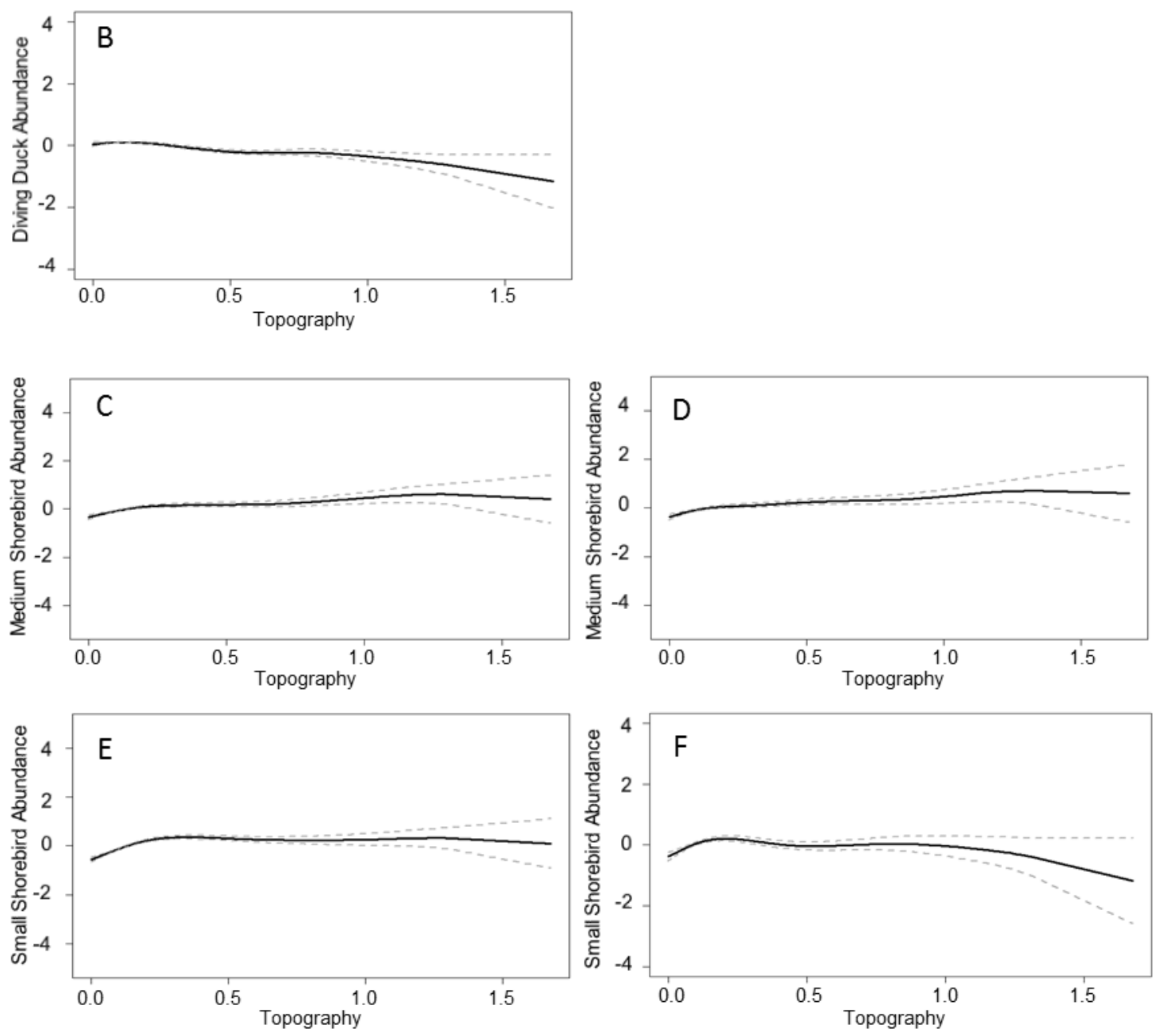

Figure 45. Dabbling duck $(A)$, diving duck $(B)$, medium $(C-D)$ and small shorebird $(E-F)$, gull $(G)$, wader $(H-l)$ abundance response to pond topography (in meters) from generalized additive models at the grid scale in restoration ponds, South San Francisco Bay, California. Dashed lines indicate 95-percent confidence interval. 
Foraging

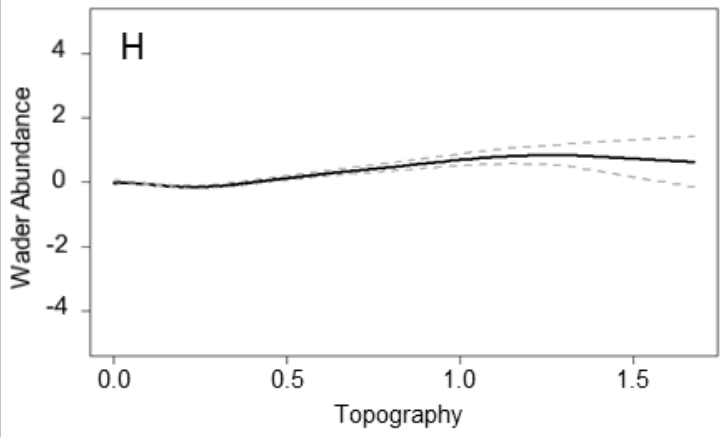

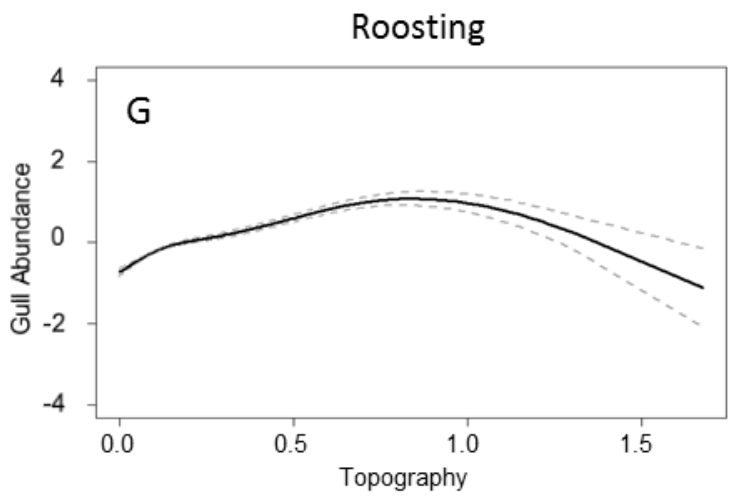

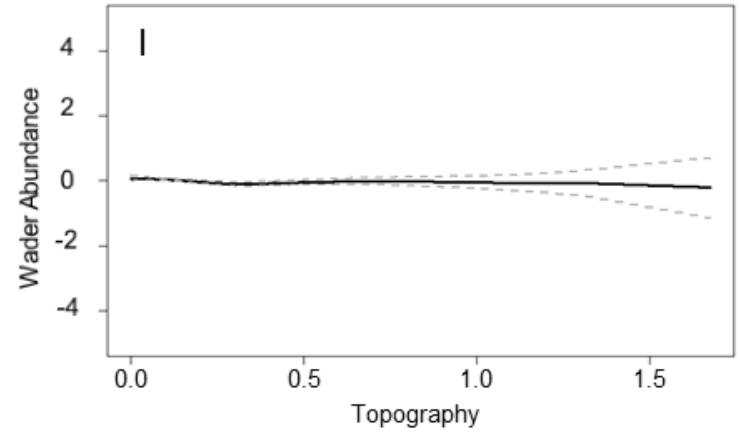

Figure 45.-Continued. 

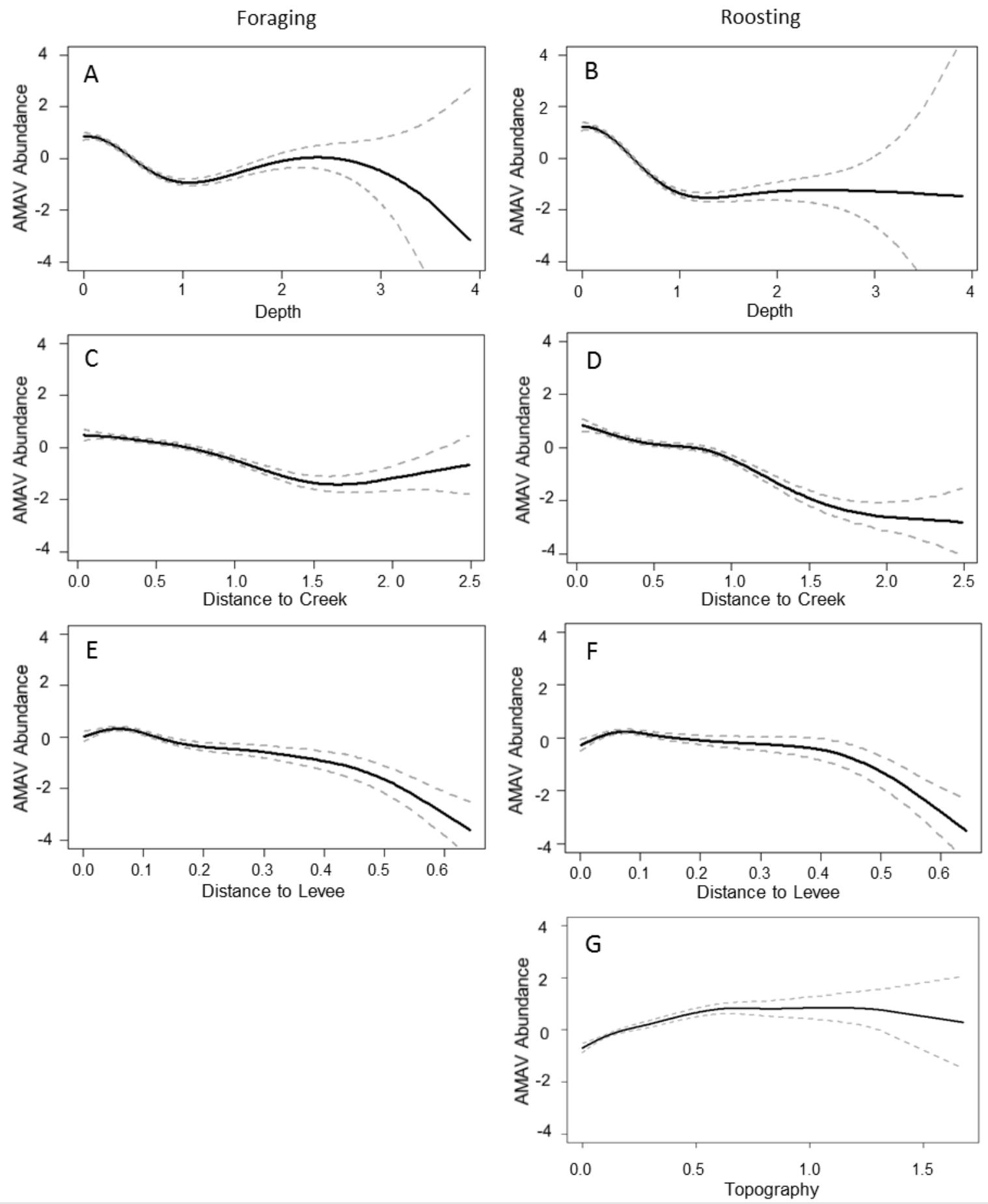

Figure 46. American avocet (AMAV) abundance response to depth (in meters relative to NAVD 88 vertical datum; A-B), distance to nearest creek (in meters; C--D), distance to levees (in meters; $E--F$ ), and pond topography (in meters; G) from generalized additive mixed models at the grid scale in restoration ponds, South San Francisco Bay, California. Dashed lines indicate the 95-percent confidence interval. 

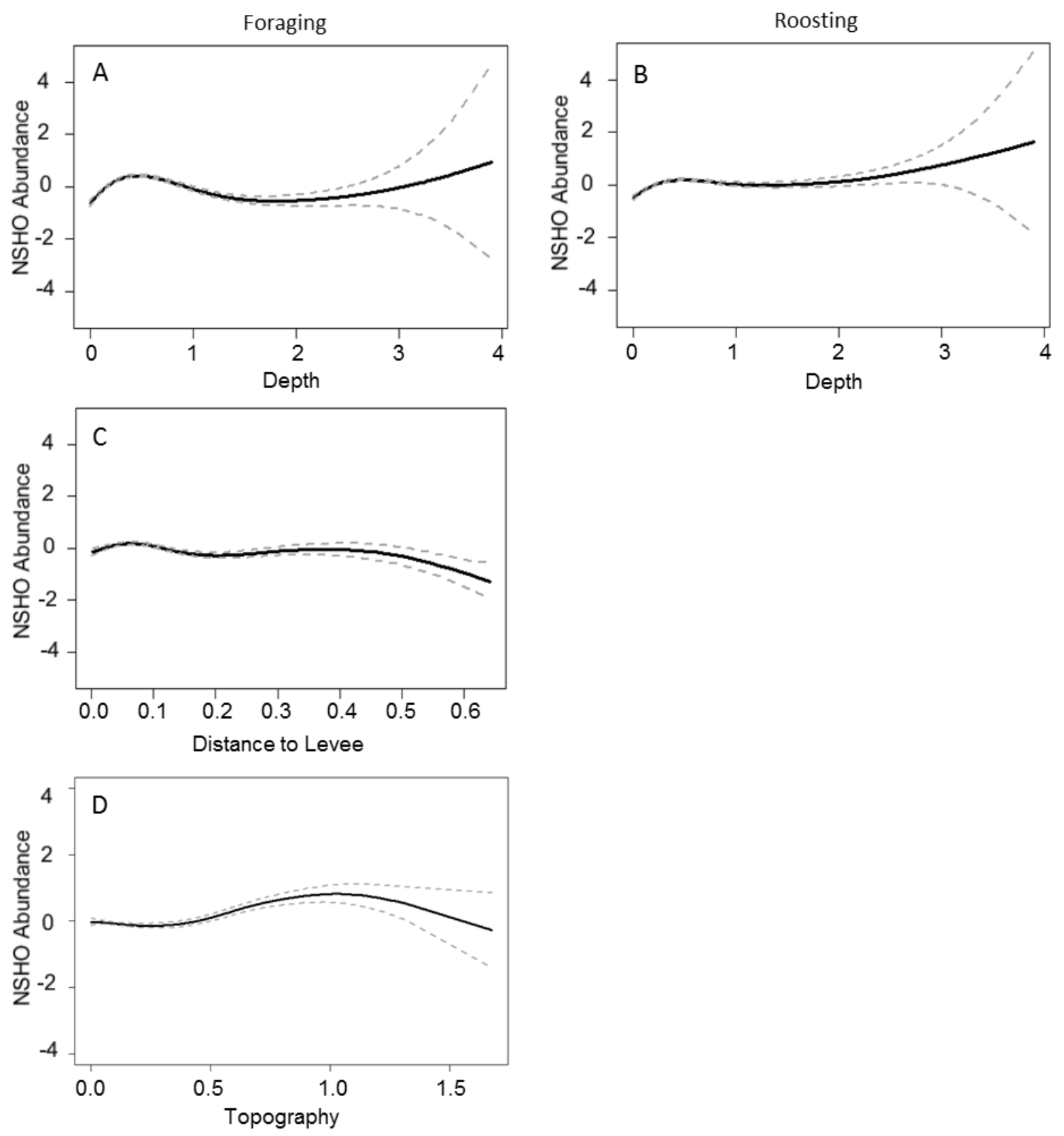

Figure 47. Northern shoveler (NSHO) abundance response to depth (in meters; $\mathrm{A}-\mathrm{B}$ ), distance to levees (in meters relative to NAVD 88 vertical datum; C), and pond topography (in meters; D) from generalized additive mixed models at the grid scale in restoration ponds, South San Francisco Bay, California. Dashed lines indicate the 95-percent confidence interval. 

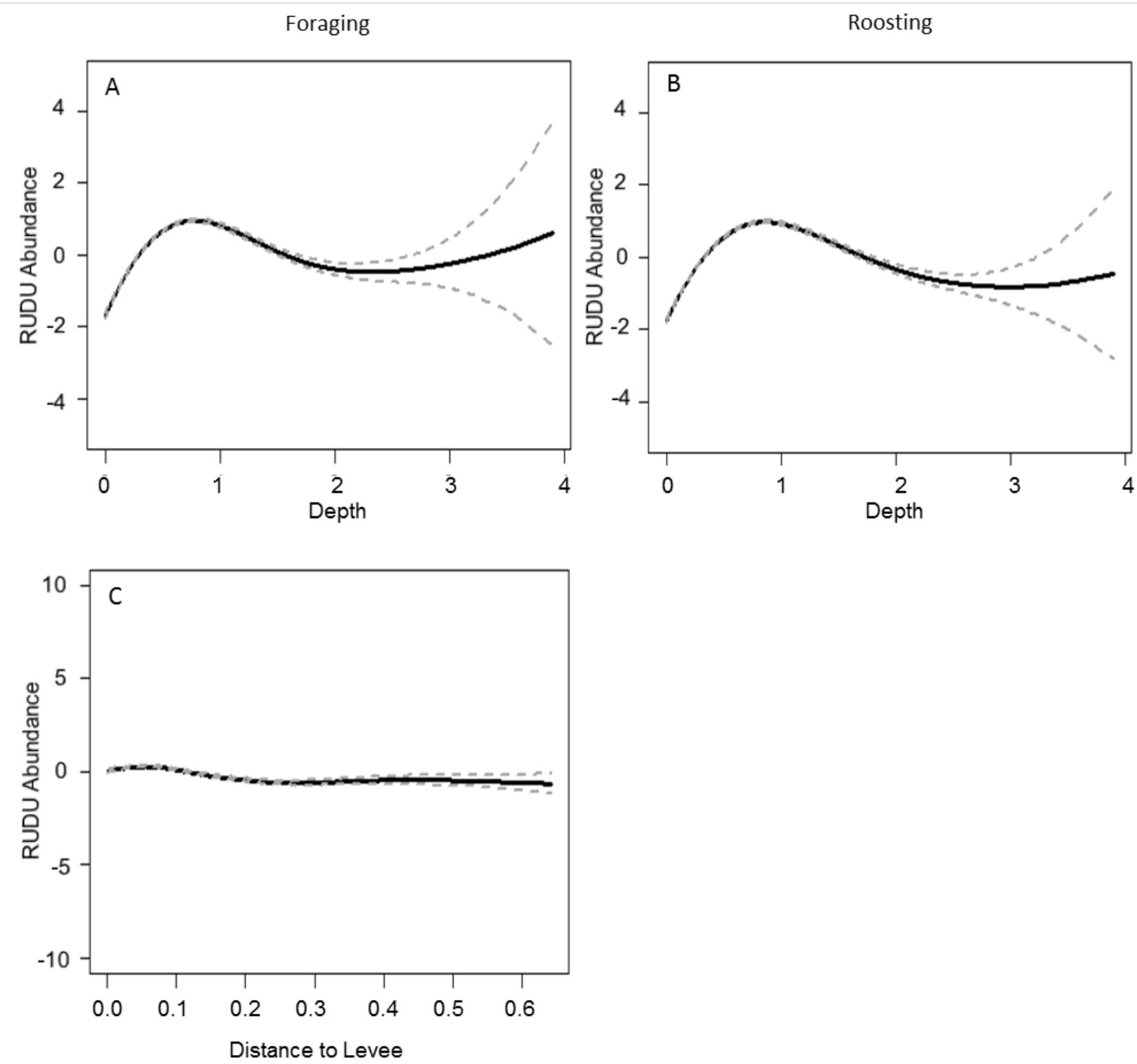

Figure 48. Ruddy duck abundance response to depth (in meters relative to NAVD 88 vertical datum; $A-B$ ) and distance to levees (in meters; $\mathrm{C}$ ) from generalized additive mixed models at the grid scale in restoration ponds, South San Francisco Bay, California. Dashed lines indicate the 95-percent confidence interval. 

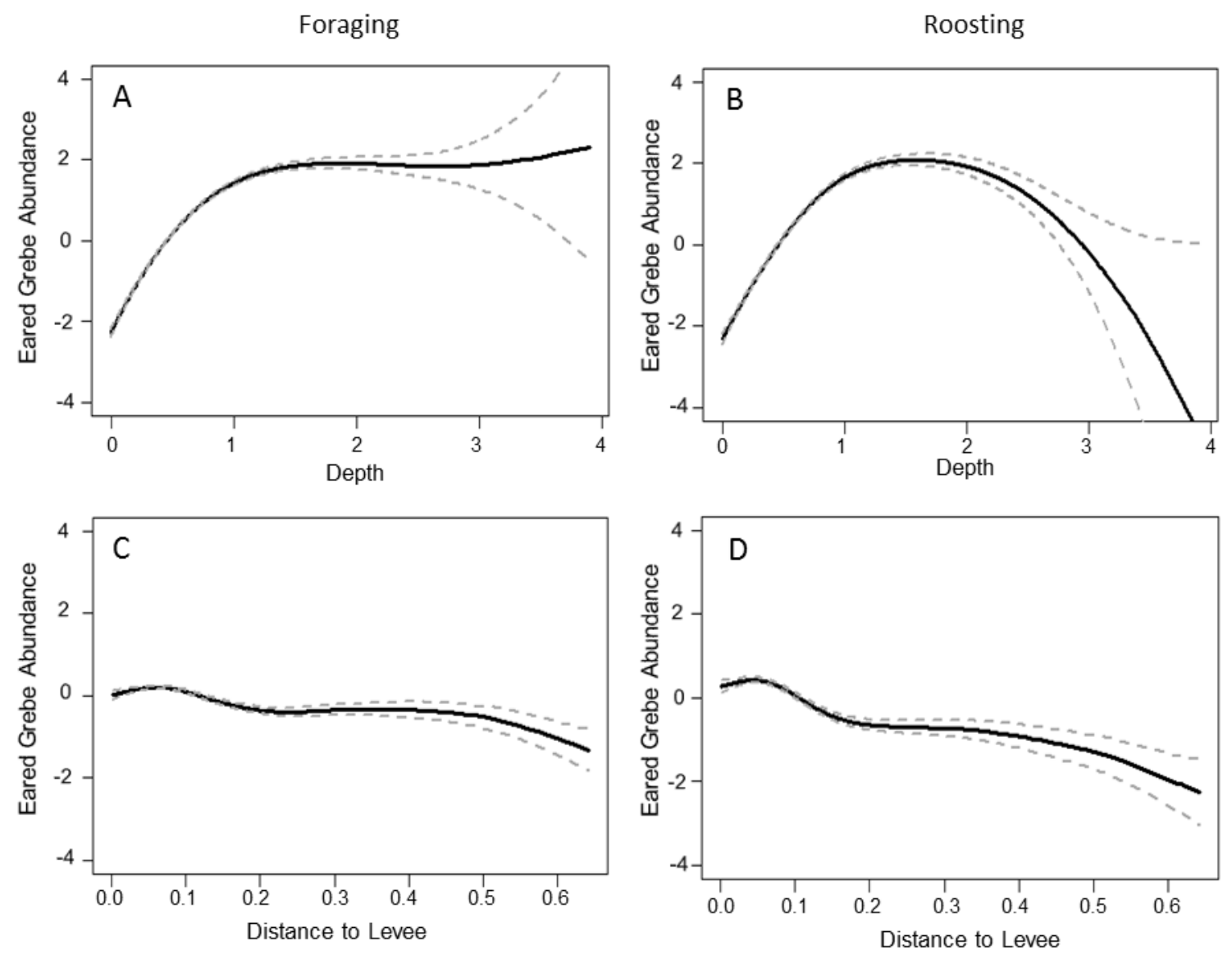

Figure 49. Eared grebe abundance response to depth (in meters relative to NAVD 88 vertical datum; A-B) and distance to levees (in meters; C-D) from generalized additive mixed models at the grid scale in restoration ponds, South San Francisco Bay, California. Dashed lines indicate the 95-percent confidence interval. 
Table 1. Attributes of the South Bay Salt Pond Restoration Project ponds used in waterbird habitat association models, South San Francisco Bay, California.

[Data were collected during 2002-15. Maximum \% levee open to public: We accounted for change in public access over time. For some ponds in some months and years this value may be lower. Maximum \% of levee open to hunting: We accounted for changes in hunter access over time and we only included levees open to hunters during the hunting season (November-January). Abbreviations and symbol: km, kilometer; $\mathrm{km}^{2}$; square kilometer; \%, percent]

\begin{tabular}{|c|c|c|c|c|c|c|c|}
\hline Pond & $\begin{array}{c}\text { Area } \\
(\mathrm{km} 2)\end{array}$ & $\begin{array}{c}\text { Bay } \\
\text { distance } \\
(\mathrm{km})\end{array}$ & $\begin{array}{l}\text { Urban } \\
\text { distance } \\
(\mathrm{km})\end{array}$ & $\begin{array}{l}\text { Landfill } \\
\text { distance } \\
(\mathrm{km})\end{array}$ & $\begin{array}{l}\text { Maximum \% } \\
\text { levee open to } \\
\text { public }\end{array}$ & $\begin{array}{l}\text { Maximum \% } \\
\text { of levee open } \\
\text { to hunting }\end{array}$ & $\begin{array}{l}\text { Number } \\
\text { of } \\
\text { islands }\end{array}$ \\
\hline A1 & 1.1 & 0.9 & 1.4 & 10.0 & 0.0 & 0.0 & 2 \\
\hline $\mathrm{A} 10^{1}$ & 1.0 & 1.9 & 3.4 & 4.9 & 0.5 & 0.0 & 0 \\
\hline $\mathrm{A} 11^{1}$ & 1.1 & 3.1 & 2.2 & 5.1 & 0.3 & 0.0 & 0 \\
\hline $\mathrm{A} 12^{1}$ & 1.3 & 4.5 & 0.8 & 6.1 & 0.7 & 0.0 & 0 \\
\hline $\mathrm{A} 13^{1}$ & 1.1 & 4.0 & 1.5 & 5.1 & 0.5 & 0.0 & 2 \\
\hline $\mathrm{A} 14^{1}$ & 1.4 & 2.7 & 2.9 & 4.1 & 0.4 & 0.0 & 0 \\
\hline A15 & 1.0 & 3.6 & 2.4 & 4.1 & 1.0 & 0.0 & 0 \\
\hline $\mathrm{A} 16^{1}$ & 1.0 & 5.1 & 1.0 & 5.5 & 1.0 & 0.0 & 5 \\
\hline A17 & 0.5 & 4.8 & 2.2 & 4.3 & 1.0 & 0.0 & 1 \\
\hline A19 & 1.1 & 5.9 & 1.3 & 4.0 & 0.0 & 1.0 & 0 \\
\hline A20 & 0.3 & 4.7 & 2.5 & 3.4 & 0.0 & 0.0 & 0 \\
\hline A 21 & 0.6 & 3.9 & 3.1 & 3.1 & 0.0 & 0.0 & 0 \\
\hline A22 & 1.1 & 5.7 & 0.6 & 2.8 & 0.2 & 0.0 & 0 \\
\hline A23 & 1.9 & 5.5 & 1.2 & 3.1 & 0.0 & 0.0 & 0 \\
\hline A2E & 1.3 & 0.8 & 1.0 & 8.3 & 0.4 & 0.4 & 0 \\
\hline $\mathrm{A} 2 \mathrm{~W}$ & 1.8 & 0.7 & 1.5 & 9.2 & 0.0 & 0.0 & 3 \\
\hline $\mathrm{A} 3 \mathrm{~N}$ & 0.7 & 0.3 & 2.3 & 6.4 & 0.0 & 0.8 & 0 \\
\hline $\mathrm{A} 3 \mathrm{~W}$ & 2.3 & 1.6 & 1.2 & 7.6 & 0.2 & 0.6 & 0 \\
\hline A5 & 2.6 & 2.4 & 1.9 & 6.3 & 0.0 & 0.7 & 1 \\
\hline A7 & 1.1 & 2.8 & 2.4 & 5.8 & 0.0 & 0.4 & 7 \\
\hline A8 & 1.7 & 4.7 & 1.0 & 6.9 & 0.0 & 0.5 & 0 \\
\hline A9* & 1.5 & 1.4 & 4.0 & 4.0 & 0.5 & 0.0 & 0 \\
\hline $\mathrm{AB} 1$ & 0.6 & 0.2 & 1.6 & 8.1 & 0.2 & 0.5 & 1 \\
\hline AB2 & 0.7 & 0.9 & 1.1 & 7.6 & 0.1 & 0.2 & 17 \\
\hline B1 & 1.2 & 1.5 & 3.1 & 16.2 & 0.0 & 1.0 & 1 \\
\hline B10 & 0.9 & 0.4 & 0.6 & 19.2 & 0.0 & 0.6 & 3 \\
\hline B11 & 0.5 & 0.9 & 0.6 & 19.0 & 0.0 & 1.0 & 0 \\
\hline B12 & 0.4 & 2.2 & 0.7 & 18.1 & 0.0 & 1.0 & 0 \\
\hline B13 & 0.6 & 1.8 & 1.1 & 18.0 & 0.0 & 1.0 & 0 \\
\hline B14 & 0.7 & 1.9 & 1.5 & 17.5 & 0.0 & 1.0 & 0 \\
\hline $\mathrm{B} 1 \mathrm{C}$ & 0.3 & 2.3 & 2.0 & 13.5 & 0.0 & 0.0 & 0 \\
\hline B2 & 2.8 & 0.8 & 3.7 & 15.7 & 0.0 & 0.8 & 6 \\
\hline B2C & 0.1 & 2.5 & 1.7 & 12.9 & 0.0 & 0.0 & 1 \\
\hline B4 & 0.8 & 2.1 & 2.3 & 14.5 & 0.0 & 0.6 & 4 \\
\hline B5 & 0.7 & 3.4 & 1.0 & 14.3 & 0.0 & 1.0 & 0 \\
\hline B5C & 0.4 & 2.9 & 1.4 & 13.1 & 0.0 & 0.0 & 0 \\
\hline B6 & 0.8 & 3.8 & 0.7 & 14.5 & 0.0 & 1.0 & 1 \\
\hline B6A & 1.3 & 4.1 & 0.5 & 15.3 & 0.0 & 0.6 & 2 \\
\hline B6B & 1.1 & 3.2 & 1.2 & 16.0 & 0.0 & 1.0 & 0 \\
\hline B6C & 0.3 & 3.1 & 1.3 & 13.8 & 0.0 & 0.2 & 0 \\
\hline B7 & 0.9 & 2.4 & 2.1 & 15.1 & 0.0 & 1.0 & 2 \\
\hline B8 & 0.8 & 2.7 & 1.9 & 15.9 & 0.0 & 1.0 & 0 \\
\hline B8AE & 0.5 & 1.8 & 2.8 & 17.1 & 0.0 & 1.0 & 0 \\
\hline B8X & 0.1 & 2.4 & 1.5 & 16.9 & 0.0 & 1.0 & 0 \\
\hline B9 & 1.5 & 1.1 & 2.2 & 17.6 & 0.0 & 1.0 & 2 \\
\hline R2 & 0.6 & 0.8 & 0.9 & 13.3 & 0.4 & 0.4 & 0 \\
\hline R3 & 1.1 & 1.3 & 0.4 & 14.5 & 0.0 & 0.0 & 0 \\
\hline
\end{tabular}




\begin{tabular}{lccccccc}
\hline Pond & $\begin{array}{c}\text { Area } \\
(\mathbf{k m})\end{array}$ & $\begin{array}{c}\text { Bay } \\
\text { distance } \\
(\mathbf{k m})\end{array}$ & $\begin{array}{c}\text { Urban } \\
\text { distance } \\
(\mathbf{k m})\end{array}$ & $\begin{array}{c}\text { Landfill } \\
\text { distance } \\
\mathbf{( k m})\end{array}$ & $\begin{array}{c}\text { Maximum \% } \\
\text { levee open to } \\
\text { public }\end{array}$ & $\begin{array}{c}\text { Maximum \% } \\
\text { of levee open } \\
\text { to hunting }\end{array}$ & $\begin{array}{c}\text { Number } \\
\text { of } \\
\text { islands }\end{array}$ \\
\hline R4 & 1.2 & 0.5 & 1.1 & 15.1 & 0.2 & 0.0 & 0 \\
R5 & 0.1 & 1.2 & 0.4 & 15.8 & 0.3 & 0.0 & 0 \\
RSF2U1 & 0.2 & 0.2 & 1.3 & 12.2 & 0.6 & 0.0 & 8 \\
RSF2U2 & 0.3 & 0.5 & 0.9 & 12.5 & 0.3 & 0.0 & 22 \\
RSF2U3 & 0.4 & 1.0 & 0.5 & 13.0 & 0.5 & 0.0 & 0 \\
\hline
\end{tabular}

${ }^{1}$ Indicates ponds surveyed in fall 2002. 
Table 2. Common name, scientific name, and associated foraging guild of waterbird species observed on salt ponds, South San Francisco Bay, California, October-April 2002-13.

[Common name: Species that were evaluated individually in modeling efforts are identified by four-letter species coding. (J) indicates species present only on Project ponds; (D) indicates species present only on Production ponds. Species for which we observed 10 or fewer individuals across the study period were excluded from this list (Project ponds $=20$ species; Production Ponds $=8$ species).]

\begin{tabular}{|c|c|c|}
\hline Common name & Scientific name & Foraging guild \\
\hline American avocet (AMAV) & Recurvirostra americana & Medium shorebird \\
\hline American coot & Fulica americana & Dabbling duck \\
\hline American green-winged teal & Anas crecca & Dabbling duck \\
\hline American white pelican & Pelecanus erythrorhynchos & Piscivore \\
\hline American wigeon & Anas americana & Dabbling duck \\
\hline Belted kingfisher & Megaceryle alcyon & Piscivore \\
\hline Black oystercatcher & Haematopus bachmani & Medium shorebird \\
\hline Black skimmer & Rynchops niger & Tern \\
\hline Black turnstone & Arenaria melanocephala & Medium shorebird \\
\hline Black-bellied plover & Pluvialis squatarola & Medium shorebird \\
\hline Black-crowned night-heron & Nycticorax nycticorax & Wader \\
\hline Black-necked stilt (BNST) & Himantopus mexicanus & Medium shorebird \\
\hline Blue-winged teal & Anas discors & Dabbling duck \\
\hline Bonaparte's gull & Chroicocephalus philadelphia & Gull \\
\hline Brown pelican & Pelecanus occidentalis & Piscivore \\
\hline Bufflehead & Bucephala albeola & Diving duck \\
\hline Cackling goose $(\mathrm{J})$ & Branta hutchinsii & Goose \\
\hline California gull & Larus californicus & Gull \\
\hline Canada goose & Branta canadensis & Goose \\
\hline Canvasback & Aythya valisineria & Diving duck \\
\hline Caspian tern & Hydroprogne caspia & Tern \\
\hline Cinnamon teal & Anas cyanoptera & Dabbling duck \\
\hline Clark's grebe & Aechmophorus clarkii & Piscivore \\
\hline Common goldeneye & Bucephala clangula & Diving duck \\
\hline Common merganser & Mergus merganser & Piscivore \\
\hline Double-crested cormorant & Phalacrocorax auritus & Piscivore \\
\hline Dunlin & Calidris alpina & Small shorebird \\
\hline Eared grebe (EAGR) & Podiceps nigricollis & Eared grebe \\
\hline Elegant tern & Thalasseus elegans & Tern \\
\hline Eurasian wigeon & Anas penelope & Dabbling duck \\
\hline Forster's tern (FOTE) & Sterna forsteri & Tern \\
\hline Gadwall & Anas strepera & Dabbling duck \\
\hline Glaucous gull & Larus hyperboreus & Gull \\
\hline Glaucous-winged gull & Larus glaucescens & Gull \\
\hline Great blue heron & Ardea herodias & Wader \\
\hline Great egret & Ardea alba & Wader \\
\hline Greater scaup & Aythya marila & Diving duck \\
\hline Greater white-fronted goose & Anser albifrons & Goose \\
\hline
\end{tabular}




\begin{tabular}{|c|c|c|}
\hline Common name & Scientific name & Foraging guild \\
\hline Greater yellowlegs & Tringa melanoleuca & Medium shorebird \\
\hline Herring gull & Larus argentatus & Gull \\
\hline Hooded merganser & Lophodytes cucullatus & Piscivore \\
\hline Horned grebe & Podiceps auritus & Eared grebe \\
\hline Killdeer & Charadrius vociferus & Medium shorebird \\
\hline Least sandpiper & Calidris minutilla & Small shorebird \\
\hline Least tern & Sternula antillarum & Tern \\
\hline Lesser scaup & Aythya affinis & Diving duck \\
\hline Lesser yellowlegs & Tringa flavipes & Medium shorebird \\
\hline Long-billed curlew & Numenius americanus & Medium shorebird \\
\hline Long-billed dowitcher & Limnodromus scolopaecus & Small shorebird \\
\hline Mallard & Anas platyrhynchos & Dabbling duck \\
\hline Marbled godwit & Limosa fedoa & Medium shorebird \\
\hline Mew gull & Larus canus & Gull \\
\hline Northern pintail & Anas acuta & Dabbling duck \\
\hline Northern shoveler & Anas clypeata & Dabbling duck \\
\hline Pectoral sandpiper (D) & Calidris melanotos & Small shorebird \\
\hline Pied-billed grebe & Podilymbus podiceps & Piscivore \\
\hline Red knot & Calidris canutus & Medium shorebird \\
\hline Red phalarope & Phalaropus fulicaria & Phalarope \\
\hline Red-breasted merganser & Mergus serrator & Piscivore \\
\hline Redhead & Aythya americana & Diving duck \\
\hline Red-necked phalarope & Phalaropus lobatus & Phalarope \\
\hline Red-throated loon & Gavia stellata & Piscivore \\
\hline Ring-billed gull & Larus delawarensis & Gull \\
\hline Ruddy duck & Oxyura jamaicensis & Diving duck \\
\hline Ruddy turnstone & Arenaria interpres & Medium shorebird \\
\hline Sabine's gull (J) & Xema sabini & Gull \\
\hline Sanderling & Calidris alba & Small shorebird \\
\hline Semipalmated plover & Charadrius semipalmatus & Small shorebird \\
\hline Short-billed dowitcher & Limnodromus griseus & Small shorebird \\
\hline Snow goose & Chen caerulescens & Goose \\
\hline Snowy egret & Egretta thula & Wader \\
\hline Snowy plover & Charadrius alexandrinus & Small shorebird \\
\hline Spotted sandpiper & Actitis macularius & Small shorebird \\
\hline Surf scoter & Melanitta perspicillata & Diving duck \\
\hline Thayer's gull & Larus thayeri & Gull \\
\hline Western grebe & Aechmophorus occidentalis & Piscivore \\
\hline Western gull & Larus occidentalis & Gull \\
\hline Western sandpiper (WESA) & Calidris mauri & Small shorebird \\
\hline Whimbrel & Numenius phaeopus & Medium shorebird \\
\hline White-winged scoter & Melanitta fusca & Diving duck \\
\hline Willet & Tringa semipalmata & Medium shorebird \\
\hline Wilson's phalarope (J) & Phalaropus tricolor & Phalarope \\
\hline
\end{tabular}


Table 3. The ranking of candidate models at the pond scale for foraging dabbling ducks in the former salt production ponds of South San Francisco Bay, California.

[Models are ranked by differences in Akaike's information criterion and only models of $\triangle \mathrm{AIC}<4$. Birds were surveyed across the former salt ponds during October through April 2002-2015. Variables considered included the mean pond depth (Depth), the presence of islands (Island), variation in topography (DepthSD), \% of pond levee open to hunting (HuntLevee), \% of pond levee open to the public (OpenLevee), pond area (Area), water salinity, pond distance to urban areas (UrbanDist), and pond distance to the bay (BayDist). k: Number of estimated parameters in the model. AICc: Second-order Akaike's information criterion. $\triangle \mathrm{AICc}$ : The difference between the AICc of the current model and the AICc of the top model. $\mathbf{w}_{\mathbf{i}}$ : Akaike weight-likelihood of the model relative to candidate models.

Evidence ratio: The weight of evidence that the current model is inferior to the top model]

\begin{tabular}{|c|c|c|c|c|c|c|}
\hline Model name & k & $-2 \log L$ & AICc & $\triangle \mathrm{AICc}$ & $w_{i}$ & $\begin{array}{c}\text { Evidence } \\
\text { ratio }\end{array}$ \\
\hline Salinity + Island + DepthSD & 8 & 25006.2 & 25022.3 & 0.00 & 0.04 & 1.00 \\
\hline Salinity + Area + OpenLevee + Island + DepthSD & 10 & 25002.6 & 25022.7 & 0.43 & 0.03 & 1.24 \\
\hline Salinity + UrbanDist + Island + DepthSD & 9 & 25005.0 & 25023.1 & 0.81 & 0.03 & 1.50 \\
\hline Salinity + Island + Breached + DepthSD & 9 & 25005.0 & 25023.1 & 0.81 & 0.03 & 1.50 \\
\hline Depth + Salinity + Island + DepthSD & 9 & 25005.2 & 25023.3 & 1.01 & 0.02 & 1.66 \\
\hline Salinity + OpenLevee + Island & 8 & 25007.6 & 25023.7 & 1.40 & 0.02 & 2.01 \\
\hline Salinity + Area + Island & 8 & 25007.8 & 25023.9 & 1.60 & 0.02 & 2.23 \\
\hline Depth + Salinity + UrbanDist + Island + DepthSD & 10 & 25003.8 & 25023.9 & 1.63 & 0.02 & 2.26 \\
\hline Depth + Salinity + Area + OpenLevee + Island & 10 & 25003.8 & 25023.9 & 1.63 & 0.02 & 2.26 \\
\hline Salinity + UrbanDist + Island + Breached + DepthSD & 10 & 25003.8 & 25023.9 & 1.63 & 0.02 & 2.26 \\
\hline Salinity + HuntLevee + Island + DepthSD & 9 & 25006.0 & 25024.1 & 1.81 & 0.02 & 2.48 \\
\hline Depth + Salinity + Area + Island + Breached + DepthSD & 11 & 25002.0 & 25024.1 & 1.84 & 0.02 & 2.51 \\
\hline Salinity + BayDist + Island + DepthSD & 9 & 25006.2 & 25024.3 & 2.01 & 0.01 & 2.74 \\
\hline Salinity + UrbanDist + OpenLevee + Island + DepthSD & 10 & 25004.2 & 25024.3 & 2.03 & 0.01 & 2.75 \\
\hline Depth + Salinity + OpenLevee + Island + DepthSD & 10 & 25004.4 & 25024.5 & 2.23 & 0.01 & 3.04 \\
\hline Depth + Salinity + Island + Breached + DepthSD & 10 & 25004.4 & 25024.5 & 2.23 & 0.01 & 3.04 \\
\hline Salinity + OpenLevee + Island + Breached + DepthSD & 10 & 25004.4 & 25024.5 & 2.23 & 0.01 & 3.04 \\
\hline Salinity + Area + OpenLevee + HuntLevee + Island + DepthSD & 11 & 25002.4 & 25024.5 & 2.24 & 0.01 & 3.07 \\
\hline Salinity + Island & 7 & 25010.6 & 25024.6 & 2.39 & 0.01 & 3.30 \\
\hline Salinity + UrbanDist + OpenLevee + Island & 9 & 25006.6 & 25024.7 & 2.41 & 0.01 & 3.34 \\
\hline Salinity + BayDist + Area + OpenLevee + Island + DepthSD & 11 & 25002.6 & 25024.7 & 2.44 & 0.01 & 3.39 \\
\hline Salinity + UrbanDist + HuntLevee + Island + DepthSD & 10 & 25004.8 & 25024.9 & 2.63 & 0.01 & 3.72 \\
\hline Salinity + BayDist + Area + Island + Breached + DepthSD & 11 & 25002.8 & 25024.9 & 2.64 & 0.01 & 3.75 \\
\hline Salinity + OpenLevee + Island + Breached & 9 & 25007.0 & 25025.1 & 2.81 & 0.01 & 4.08 \\
\hline Salinity + BayDist + UrbanDist + Island + DepthSD & 10 & 25005.0 & 25025.1 & 2.83 & 0.01 & 4.11 \\
\hline Salinity + BayDist + Island + Breached + DepthSD & 10 & 25005.0 & 25025.1 & 2.83 & 0.01 & 4.11 \\
\hline Salinity + HuntLevee + Island + Breached + DepthSD & 10 & 25005.0 & 25025.1 & 2.83 & 0.01 & 4.11 \\
\hline Depth + Salinity + UrbanDist + Island + Breached + DepthSD & 11 & 25003.0 & 25025.1 & 2.84 & 0.01 & 4.14 \\
\hline Depth + Salinity + UrbanDist + Area + OpenLevee + Island + DepthSD & 12 & 25001.0 & 25025.1 & 2.86 & 0.01 & 4.18 \\
\hline Depth + Salinity + OpenLevee + Island & 9 & 25007.2 & 25025.3 & 3.01 & 0.01 & 4.51 \\
\hline Depth + Salinity + BayDist + Island + DepthSD & 10 & 25005.2 & 25025.3 & 3.03 & 0.01 & 4.54 \\
\hline Depth + Salinity + HuntLevee + Island + DepthSD & 10 & 25005.2 & 25025.3 & 3.03 & 0.01 & 4.54 \\
\hline Salinity + BayDist + OpenLevee + Island + DepthSD & 10 & 25005.2 & 25025.3 & 3.03 & 0.01 & 4.54 \\
\hline Salinity + OpenLevee + HuntLevee + Island + DepthSD & 10 & 25005.2 & 25025.3 & 3.03 & 0.01 & 4.54 \\
\hline Depth + Salinity + UrbanDist + OpenLevee + Island + DepthSD & 11 & 25003.2 & 25025.3 & 3.04 & 0.01 & 4.58 \\
\hline Salinity + BayDist + UrbanDist + Area + Island + DepthSD & 11 & 25003.2 & 25025.3 & 3.04 & 0.01 & 4.58 \\
\hline Depth + Salinity + Area + OpenLevee + Island + Breached + DepthSD & 12 & 25001.2 & 25025.3 & 3.06 & 0.01 & 4.62 \\
\hline Salinity + BayDist + OpenLevee + Island & 9 & 25007.4 & 25025.5 & 3.21 & 0.01 & 4.98 \\
\hline
\end{tabular}




\begin{tabular}{|c|c|c|c|c|c|c|}
\hline Model name & k & $-2 \log L$ & $\mathrm{AICc}$ & $\triangle \mathrm{AlCc}$ & $\mathbf{W}_{\mathbf{i}}$ & $\begin{array}{c}\text { Evidence } \\
\text { ratio }\end{array}$ \\
\hline Salinity + OpenLevee + HuntLevee + Island & 9 & 25007.4 & 25025.5 & 3.21 & 0.01 & 4.98 \\
\hline Salinity + UrbanDist + OpenLevee + Island + Breached + DepthSD & 11 & 25003.4 & 25025.5 & 3.24 & 0.01 & 5.06 \\
\hline Depth + Salinity + Area + OpenLevee + HuntLevee + Island + DepthSD & 12 & 25001.4 & 25025.5 & 3.26 & 0.01 & 5.10 \\
\hline Salinity + UrbanDist + Area + OpenLevee + Island + Breached + DepthSD & 12 & 25001.4 & 25025.5 & 3.26 & 0.01 & 5.10 \\
\hline Salinity + UrbanDist + Island & 8 & 25009.6 & 25025.7 & 3.40 & 0.01 & 5.47 \\
\hline Salinity + Island + Breached & 8 & 25009.6 & 25025.7 & 3.40 & 0.01 & 5.47 \\
\hline Depth + Salinity + BayDist + Area + OpenLevee + Island & 11 & 25003.6 & 25025.7 & 3.44 & 0.01 & 5.59 \\
\hline Depth + Salinity + Area + OpenLevee + Island + Breached & 11 & 25003.6 & 25025.7 & 3.44 & 0.01 & 5.59 \\
\hline Salinity + BayDist + Area + HuntLevee + Island + DepthSD & 11 & 25003.6 & 25025.7 & 3.44 & 0.01 & 5.59 \\
\hline Salinity + UrbanDist + HuntLevee + Island + Breached + DepthSD & 11 & 25003.6 & 25025.7 & 3.44 & 0.01 & 5.59 \\
\hline Depth + Salinity + BayDist + Area + OpenLevee + Island + DepthSD & 12 & 25001.6 & 25025.7 & 3.46 & 0.01 & 5.64 \\
\hline Depth + Salinity + BayDist + UrbanDist + Island + DepthSD & 11 & 25003.8 & 25025.9 & 3.64 & 0.01 & 6.18 \\
\hline Depth + Salinity + UrbanDist + HuntLevee + Island + DepthSD & 11 & 25003.8 & 25025.9 & 3.64 & 0.01 & 6.18 \\
\hline Depth + Salinity + Area + OpenLevee + HuntLevee + Island & 11 & 25003.8 & 25025.9 & 3.64 & 0.01 & 6.18 \\
\hline Depth + Salinity + OpenLevee + Island + Breached + DepthSD & 11 & 25003.8 & 25025.9 & 3.64 & 0.01 & 6.18 \\
\hline Salinity + BayDist + UrbanDist + Island + Breached + DepthSD & 11 & 25003.8 & 25025.9 & 3.64 & 0.01 & 6.18 \\
\hline Salinity + BayDist + Area + OpenLevee + Island + Breached & 11 & 25003.8 & 25025.9 & 3.64 & 0.01 & 6.18 \\
\hline Depth + Salinity + UrbanDist + Area + HuntLevee + Island + DepthSD & 12 & 25001.8 & 25025.9 & 3.66 & 0.01 & 6.23 \\
\hline Depth + Salinity + Area + HuntLevee + Island + Breached + DepthSD & 12 & 25001.8 & 25025.9 & 3.66 & 0.01 & 6.23 \\
\hline Salinity + BayDist + Area + OpenLevee + Island + Breached + DepthSD & 12 & 25001.8 & 25025.9 & 3.66 & 0.01 & 6.23 \\
\hline Salinity + BayDist + Island & 8 & 25010.0 & 25026.1 & 3.80 & 0.01 & 6.69 \\
\hline Salinity + BayDist + HuntLevee + Island + DepthSD & 10 & 25006.0 & 25026.1 & 3.83 & 0.01 & 6.78 \\
\hline Salinity + UrbanDist + OpenLevee + Island + Breached & 10 & 25006.0 & 25026.1 & 3.83 & 0.01 & 6.78 \\
\hline Salinity + UrbanDist + OpenLevee + HuntLevee + Island + DepthSD & 11 & 25004.0 & 25026.1 & 3.84 & 0.01 & 6.83 \\
\hline
\end{tabular}


Table 4. The ranking of candidate models at the pond scale for roosting dabbling ducks in the former salt production ponds of South San Francisco Bay, California.

[Models are ranked by differences in Akaike's information criterion and only models of $\triangle \mathrm{AIC}<4$ and the null model are presented. Birds were surveyed across the former salt ponds during October through April 2002-2015. Variables considered included the mean pond depth (Depth), the presence of islands (Island), variation in topography (DepthSD), \% of pond levee open to hunting (HuntLevee), \% of pond levee open to the public (OpenLevee), pond area (Area), water salinity, pond distance to urban areas (UrbanDist), and pond distance to the bay (BayDist). k: Number of estimated parameters in the model. AICc: Second-order Akaike's information criterion. $\triangle$ AICc: The difference between the AICc of the current model and the AICc of the top model. wi: Akaike weight-likelihood of the model relative to candidate models. Evidence ratio: The weight of evidence that the current model is inferior to the top model]

\begin{tabular}{|c|c|c|c|c|c|c|}
\hline Model name & k & $-2 \log L$ & AICc & $\triangle \mathrm{AICc}$ & $\mathbf{w}_{\mathrm{i}}$ & $\begin{array}{c}\text { Evidence } \\
\text { ratio }\end{array}$ \\
\hline Salinity + Area + HuntLevee + Island + DepthSD & 10 & 27679.0 & 27699.1 & 0.00 & 0.08 & 1.00 \\
\hline Salinity + UrbanDist + Area + HuntLevee + Island + DepthSD & 11 & 27677.4 & 27699.5 & 0.42 & 0.07 & 1.23 \\
\hline Salinity + BayDist + Area + HuntLevee + Island + DepthSD & 11 & 27678.0 & 27700.1 & 1.02 & 0.05 & 1.66 \\
\hline Depth + Salinity + Area + HuntLevee + Island + DepthSD & 11 & 27678.2 & 27700.3 & 1.22 & 0.04 & 1.84 \\
\hline Salinity + UrbanDist + HuntLevee + Island + DepthSD & 10 & 27680.4 & 27700.5 & 1.40 & 0.04 & 2.01 \\
\hline Salinity + Area + HuntLevee + Island + Breached + DepthSD & 11 & 27678.6 & 27700.7 & 1.62 & 0.04 & 2.24 \\
\hline Salinity + BayDist + UrbanDist + Area + HuntLevee + Island + DepthSD & 12 & 27676.8 & 27700.9 & 1.83 & 0.03 & 2.50 \\
\hline Salinity + HuntLevee + Island + DepthSD & 9 & 27683.0 & 27701.1 & 1.99 & 0.03 & 2.70 \\
\hline Salinity + Area + OpenLevee + HuntLevee + Island + DepthSD & 11 & 27679.0 & 27701.1 & 2.02 & 0.03 & 2.74 \\
\hline Salinity + UrbanDist + Area + HuntLevee + Island + Breached + DepthSD & 12 & 27677.2 & 27701.3 & 2.23 & 0.03 & 3.05 \\
\hline Depth + Salinity + BayDist + Area + HuntLevee + Island + DepthSD & 12 & 27677.4 & 27701.5 & 2.43 & 0.02 & 3.37 \\
\hline \multicolumn{7}{|l|}{ Salinity + UrbanDist + Area + OpenLevee + HuntLevee + Island +} \\
\hline DepthSD & 12 & 27677.4 & 27701.5 & 2.43 & 0.02 & 3.37 \\
\hline Depth + Salinity + UrbanDist + HuntLevee + Island + DepthSD & 11 & 27679.6 & 27701.7 & 2.62 & 0.02 & 3.70 \\
\hline Salinity + BayDist + Area + HuntLevee + Island + Breached + DepthSD & 12 & 27677.6 & 27701.7 & 2.63 & 0.02 & 3.73 \\
\hline Depth + Salinity + Area + HuntLevee + Island + Breached + DepthSD & 12 & 27677.8 & 27701.9 & 2.83 & 0.02 & 4.12 \\
\hline Salinity + BayDist + UrbanDist + HuntLevee + Island + DepthSD & 11 & 27680.0 & 27702.1 & 3.02 & 0.02 & 4.52 \\
\hline Salinity + BayDist + Area + OpenLevee + HuntLevee + Island + DepthSD & 12 & 27678.0 & 27702.1 & 3.03 & 0.02 & 4.55 \\
\hline \multicolumn{7}{|l|}{ Depth + Salinity + BayDist + UrbanDist + Area + HuntLevee + Island +} \\
\hline DepthSD & 13 & 27676.0 & 27702.1 & 3.05 & 0.02 & 4.60 \\
\hline \multicolumn{7}{|l|}{ Depth + Salinity + UrbanDist + Area + HuntLevee + Island + Breached +} \\
\hline DepthSD & 13 & 27676.0 & 27702.1 & 3.05 & 0.02 & 4.60 \\
\hline Salinity + UrbanDist + HuntLevee + Island + Breached + DepthSD & 11 & 27680.2 & 27702.3 & 3.22 & 0.02 & 4.99 \\
\hline Depth + Salinity + Area + OpenLevee + HuntLevee + Island + DepthSD & 12 & 27678.2 & 27702.3 & 3.23 & 0.02 & 5.03 \\
\hline Depth + Salinity + HuntLevee + Island + DepthSD & 10 & 27682.4 & 27702.5 & 3.40 & 0.01 & 5.47 \\
\hline Salinity + BayDist + HuntLevee + Island + DepthSD & 10 & 27682.4 & 27702.5 & 3.40 & 0.01 & 5.47 \\
\hline Salinity + UrbanDist + OpenLevee + HuntLevee + Island + DepthSD & 11 & 27680.4 & 27702.5 & 3.42 & 0.01 & 5.52 \\
\hline \multicolumn{7}{|l|}{ Salinity + BayDist + UrbanDist + Area + HuntLevee + Island + Breached +} \\
\hline DepthSD & 13 & 27676.4 & 27702.5 & 3.45 & 0.01 & 5.61 \\
\hline Salinity + Area + OpenLevee + HuntLevee + Island + Breached + DepthSD & 12 & 27678.6 & 27702.7 & 3.63 & 0.01 & 6.15 \\
\hline \multicolumn{7}{|l|}{ Depth + Salinity + UrbanDist + Area + OpenLevee + HuntLevee + Island +} \\
\hline DepthSD & 13 & 27676.6 & 27702.7 & 3.65 & 0.01 & 6.20 \\
\hline Salinity + HuntLevee + Island + Breached + DepthSD & 10 & 27682.8 & 27702.9 & 3.80 & 0.01 & 6.69 \\
\hline \multicolumn{7}{|l|}{ Depth + Salinity + BayDist + Area + HuntLevee + Island + Breached +} \\
\hline DepthSD & 13 & 27676.8 & 27702.9 & 3.85 & 0.01 & 6.86 \\
\hline \multicolumn{7}{|l|}{ Salinity + BayDist + UrbanDist + Area + OpenLevee + HuntLevee + Island } \\
\hline+ DepthSD & 13 & 27676.8 & 27702.9 & 3.85 & 0.01 & 6.86 \\
\hline Null Model & 5 & 27976.6 & 27986.6 & 287.54 & $2.97 \mathrm{E}-64$ & $2.75 \mathrm{E}+62$ \\
\hline
\end{tabular}


Table 5. The ranking of candidate models at the pond scale for foraging and roosting diving ducks in the former salt production ponds of South San Francisco Bay, California.

[Models are ranked by differences in Akaike's information criterion and only models of $\triangle \mathrm{AIC}<4$ and the null model are presented. Birds were surveyed across the former salt ponds during October through April 2002-2015. Variables considered included the mean pond depth (Depth), the presence of islands (Island), variation in topography (DepthSD), \% of pond levee open to hunting (HuntLevee), \% of pond levee open to the public (OpenLevee), pond area (Area), water salinity, pond distance to urban areas (UrbanDist), and pond distance to the bay (BayDist). k: Number of estimated parameters in the model. AICc: Second-order Akaike's information criterion. $\triangle$ AICc: The difference between the AICc of the current model and the AICc of the top model. wi: Akaike weight-likelihood of the model relative to candidate models. Evidence ratio: The weight of evidence that the current model is inferior to the top model]

\begin{tabular}{|c|c|c|c|c|c|c|}
\hline Model name & k & $-2 \log L$ & AICc & $\triangle \mathrm{AICc}$ & $\mathbf{w}_{\mathrm{i}}$ & $\begin{array}{c}\text { Evidence } \\
\text { ratio }\end{array}$ \\
\hline \multicolumn{7}{|l|}{ Foraging } \\
\hline Depth + Salinity + BayDist + Area + Island + Breached & 11 & 22517.6 & 22539.7 & 0.00 & 0.24 & 1.00 \\
\hline Depth + Salinity + BayDist + Area + Island + Breached + DepthSD & 12 & 22517.0 & 22541.1 & 1.42 & 0.12 & 2.03 \\
\hline Depth + Salinity + BayDist + UrbanDist + Area + Island + Breached & 12 & 22517.6 & 22541.7 & 2.02 & 0.09 & 2.74 \\
\hline Depth + Salinity + BayDist + Area + OpenLevee + Island + Breached & 12 & 22517.6 & 22541.7 & 2.02 & 0.09 & 2.74 \\
\hline Depth + Salinity + BayDist + Area + HuntLevee + Island + Breached & 12 & 22517.6 & 22541.7 & 2.02 & 0.09 & 2.74 \\
\hline \multicolumn{7}{|l|}{ Depth + Salinity + BayDist + UrbanDist + Area + Island + Breached +} \\
\hline DepthSD & 13 & 22516.8 & 22542.9 & 3.23 & 0.05 & 5.04 \\
\hline \multicolumn{7}{|l|}{ Depth + Salinity + BayDist + Area + OpenLevee + Island + Breached +} \\
\hline DepthSD & 13 & 22517.0 & 22543.1 & 3.43 & 0.04 & 5.57 \\
\hline \multicolumn{7}{|l|}{ Depth + Salinity + BayDist + Area + HuntLevee + Island + Breached +} \\
\hline DepthSD & 13 & 22517.0 & 22543.1 & 3.43 & 0.04 & 5.57 \\
\hline \multicolumn{7}{|l|}{ Depth + Salinity + BayDist + UrbanDist + Area + OpenLevee + Island +} \\
\hline Breached & 13 & 22517.4 & 22543.5 & 3.83 & 0.04 & 6.80 \\
\hline Null Model & 5 & 22718.0 & 22728.0 & 188.33 & $3.04 \mathrm{E}-42$ & $7.85 \mathrm{E}+40$ \\
\hline \multicolumn{7}{|l|}{ Roosting } \\
\hline Depth + Salinity + BayDist + Area + HuntLevee + Island + Breached & 12 & 29025.0 & 29049.1 & 0.00 & 0.18 & 1.00 \\
\hline Depth + Salinity + BayDist + Area + Island + Breached & 11 & 29027.6 & 29049.7 & 0.58 & 0.13 & 1.34 \\
\hline Depth + Salinity + BayDist + UrbanDist + Area + Island + Breached & 12 & 29026.4 & 29050.5 & 1.40 & 0.09 & 2.01 \\
\hline \multicolumn{7}{|l|}{ Depth + Salinity + BayDist + Area + HuntLevee + Island + Breached +} \\
\hline DepthSD & 13 & 29024.4 & 29050.5 & 1.42 & 0.09 & 2.03 \\
\hline \multicolumn{7}{|l|}{ Depth + Salinity + BayDist + Area + OpenLevee + HuntLevee + Island +} \\
\hline Breached & 13 & 29024.6 & 29050.7 & 1.62 & 0.08 & 2.25 \\
\hline Depth + Salinity + BayDist + Area + Island + Breached + DepthSD & 12 & 29026.8 & 29050.9 & 1.80 & 0.07 & 2.46 \\
\hline Depth + Salinity + BayDist + Area + OpenLevee + Island + Breached & 12 & 29027.2 & 29051.3 & 2.20 & 0.06 & 3.00 \\
\hline \multicolumn{7}{|l|}{ Depth + Salinity + BayDist + UrbanDist + Area + HuntLevee + Island +} \\
\hline Breached + DepthSD & 14 & 29023.2 & 29051.4 & 2.24 & 0.06 & 3.06 \\
\hline \multicolumn{7}{|l|}{ Depth + Salinity + BayDist + UrbanDist + Area + Island + Breached +} \\
\hline DepthSD & 13 & 29025.6 & 29051.7 & 2.62 & 0.05 & 3.70 \\
\hline \multicolumn{7}{|l|}{ Depth + Salinity + BayDist + UrbanDist + Area + OpenLevee +} \\
\hline HuntLevee + Island + Breached & 14 & 29023.6 & 29051.8 & 2.64 & 0.05 & 3.74 \\
\hline \multicolumn{7}{|l|}{ Depth + Salinity + BayDist + UrbanDist + Area + OpenLevee + Island +} \\
\hline Breached & 13 & 29026.0 & 29052.1 & 3.02 & 0.04 & 4.52 \\
\hline \multicolumn{7}{|l|}{ Depth + Salinity + BayDist + Area + OpenLevee + HuntLevee + Island +} \\
\hline Breached + DepthSD & 14 & 29024.4 & 29052.6 & 3.44 & 0.03 & 5.58 \\
\hline \multicolumn{7}{|l|}{ Depth + Salinity + BayDist + Area + OpenLevee + Island + Breached +} \\
\hline DepthSD & 13 & 29026.8 & 29052.9 & 3.82 & 0.03 & 6.75 \\
\hline Null Model & 5 & 29462.6 & 29472.6 & 423.51 & $1.95 \mathrm{E}-93$ & $9.21 \mathrm{E}+91$ \\
\hline
\end{tabular}


Table 6. The ranking of candidate models at the pond scale for foraging small shorebirds in the former salt production ponds of South San Francisco Bay, California.

[Models are ranked by differences in Akaike's information criterion and only models of $\triangle \mathrm{AIC}<4$ and the null model are presented. Birds were surveyed across the former salt ponds during October through April 2002-2015. Variables considered included the mean pond depth (Depth), the presence of islands (Island), variation in topography (DepthSD), \% of pond levee open to hunting (HuntLevee), \% of pond levee open to the public (OpenLevee), pond area (Area), water salinity, pond distance to urban areas (UrbanDist), and pond distance to the bay (BayDist). k: Number of estimated parameters in the model. AICc: Second-order Akaike's information criterion. $\triangle$ AICc: The difference between the AICc of the current model and the AICc of the top model. $\mathbf{w}_{\mathbf{i}}$ : Akaike weight-likelihood of the model relative to candidate models. Evidence ratio: The weight of evidence that the current model is inferior to the top model]

\begin{tabular}{|c|c|c|c|c|c|c|}
\hline Model name & k & $-2 \log L$ & AICc & $\triangle \mathrm{AICc}$ & wi & $\begin{array}{c}\text { Evidence } \\
\text { ratio }\end{array}$ \\
\hline Depth + Salinity + OpenLevee + Island + Breached & 10 & 27025.8 & 27045.9 & 0.00 & 0.10 & 1.00 \\
\hline Depth + Salinity + BayDist + OpenLevee + Island + Breached & 11 & 27024.8 & 27046.9 & 1.02 & 0.06 & 1.66 \\
\hline Depth + Salinity + OpenLevee + Island + Breached + DepthSD & 11 & 27025.0 & 27047.1 & 1.22 & 0.05 & 1.84 \\
\hline Depth + Salinity + Area + OpenLevee + Island + Breached & 11 & 27025.2 & 27047.3 & 1.42 & 0.05 & 2.03 \\
\hline Depth + OpenLevee + Island + Breached & 9 & 27029.4 & 27047.5 & 1.59 & 0.04 & 2.21 \\
\hline Depth + Salinity + UrbanDist + OpenLevee + Island + Breached & 11 & 27025.6 & 27047.7 & 1.82 & 0.04 & 2.48 \\
\hline Depth + Salinity + OpenLevee + HuntLevee + Island + Breached & 11 & 27025.8 & 27047.9 & 2.02 & 0.04 & 2.74 \\
\hline Depth + BayDist + OpenLevee + Island + Breached & 10 & 27028.2 & 27048.3 & 2.40 & 0.03 & 3.32 \\
\hline Depth + Salinity + BayDist + Area + OpenLevee + Island + Breached & 12 & 27024.2 & 27048.3 & 2.43 & 0.03 & 3.37 \\
\hline Depth + Salinity + BayDist + OpenLevee + Island + Breached + DepthSD & 12 & 27024.4 & 27048.5 & 2.63 & 0.03 & 3.73 \\
\hline Depth + Salinity + BayDist + UrbanDist + OpenLevee + Island + Breached & 12 & 27024.6 & 27048.7 & 2.83 & 0.02 & 4.12 \\
\hline Depth + Salinity + Area + OpenLevee + Island + Breached + DepthSD & 12 & 27024.6 & 27048.7 & 2.83 & 0.02 & 4.12 \\
\hline \multicolumn{7}{|l|}{ Depth + Salinity + BayDist + OpenLevee + HuntLevee + Island +} \\
\hline Breached & 12 & 27024.8 & 27048.9 & 3.03 & 0.02 & 4.55 \\
\hline Depth + Salinity + UrbanDist + Area + OpenLevee + Island + Breached & 12 & 27024.8 & 27048.9 & 3.03 & 0.02 & 4.55 \\
\hline \multicolumn{7}{|l|}{ Depth + Salinity + UrbanDist + OpenLevee + Island + Breached +} \\
\hline DepthSD & 12 & 27024.8 & 27048.9 & 3.03 & 0.02 & 4.55 \\
\hline Depth + Area + OpenLevee + Island + Breached & 10 & 27029.0 & 27049.1 & 3.20 & 0.02 & 4.95 \\
\hline \multicolumn{7}{|l|}{ Depth + Salinity + OpenLevee + HuntLevee + Island + Breached +} \\
\hline DepthSD & 12 & 27025.0 & 27049.1 & 3.23 & 0.02 & 5.03 \\
\hline Depth + UrbanDist + OpenLevee + Island + Breached & 10 & 27029.2 & 27049.3 & 3.40 & 0.02 & 5.47 \\
\hline Depth + Salinity + Area + OpenLevee + HuntLevee + Island + Breached & 12 & 27025.2 & 27049.3 & 3.43 & 0.02 & 5.56 \\
\hline Depth + OpenLevee + HuntLevee + Island + Breached & 10 & 27029.4 & 27049.5 & 3.60 & 0.02 & 6.05 \\
\hline \multicolumn{7}{|l|}{ Depth + Salinity + UrbanDist + OpenLevee + HuntLevee + Island +} \\
\hline Breached & 12 & 27025.6 & 27049.7 & 3.83 & 0.01 & 6.79 \\
\hline Null Model & 5 & 27319.6 & 27329.6 & 283.74 & $2.37 \mathrm{E}-63$ & $4.11 \mathrm{E}+61$ \\
\hline
\end{tabular}


Table 7. The ranking of candidate models at the pond scale for roosting small shorebirds in the former salt production ponds of South San Francisco Bay, California.

[Models are ranked by differences in Akaike's information criterion and only models of $\triangle \mathrm{AIC}<4$ and the null model are presented. Birds were surveyed across the former salt ponds during October through April 2002-2015. Variables considered included the mean pond depth (Depth), the presence of islands (Island), variation in topography (DepthSD), \% of pond levee open to hunting (HuntLevee), \% of pond levee open to the public (OpenLevee), pond area (Area), water salinity, pond distance to urban areas (UrbanDist), and pond distance to the bay (BayDist). k: Number of estimated parameters in the model. AICc: Second-order Akaike's information criterion. $\triangle$ AICc: The difference between the AICc of the current model and the AICc of the top model. wi: Akaike weight-likelihood of the model relative to candidate models. Evidence ratio: The weight of evidence that the current model is inferior to the top model]

\begin{tabular}{|c|c|c|c|c|c|c|}
\hline Model name & k & $-2 \log L$ & AICc & $\triangle \mathrm{AICc}$ & $\mathbf{w}_{\mathbf{i}}$ & $\begin{array}{c}\text { Evidence } \\
\text { ratio }\end{array}$ \\
\hline Depth + Area + OpenLevee + Island + Breached & 10 & 26771.8 & 26791.9 & 0.00 & 0.03 & 1.00 \\
\hline Depth + Area + OpenLevee + HuntLevee + Island + Breached & 11 & 26769.8 & 26791.9 & 0.02 & 0.03 & 1.01 \\
\hline Depth + OpenLevee + Island + Breached & 9 & 26774.2 & 26792.3 & 0.39 & 0.02 & 1.21 \\
\hline Depth + OpenLevee + HuntLevee + Island + Breached + DepthSD & 11 & 26770.2 & 26792.3 & 0.42 & 0.02 & 1.23 \\
\hline Depth + Area + OpenLevee + HuntLevee + Island + Breached + DepthSD & 12 & 26768.2 & 26792.3 & 0.43 & 0.02 & 1.24 \\
\hline Depth + Island + Breached + DepthSD & 9 & 26774.4 & 26792.5 & 0.59 & 0.02 & 1.34 \\
\hline Depth + OpenLevee + Island + Breached + DepthSD & 10 & 26772.4 & 26792.5 & 0.60 & 0.02 & 1.35 \\
\hline Depth + HuntLevee + Island + Breached + DepthSD & 10 & 26772.4 & 26792.5 & 0.60 & 0.02 & 1.35 \\
\hline Depth + BayDist + OpenLevee + HuntLevee + Island + Breached & 11 & 26770.4 & 26792.5 & 0.62 & 0.02 & 1.36 \\
\hline Depth + Area + OpenLevee + Island + Breached + DepthSD & 11 & 26770.4 & 26792.5 & 0.62 & 0.02 & 1.36 \\
\hline Depth + BayDist + Area + OpenLevee + HuntLevee + Island + Breached & 12 & 26768.4 & 26792.5 & 0.63 & 0.02 & 1.37 \\
\hline Depth + BayDist + OpenLevee + Island + Breached & 10 & 26772.6 & 26792.7 & 0.80 & 0.02 & 1.49 \\
\hline Depth + BayDist + Area + OpenLevee + Island + Breached & 11 & 26770.6 & 26792.7 & 0.82 & 0.02 & 1.50 \\
\hline Depth + Area + HuntLevee + Island + Breached + DepthSD & 11 & 26770.8 & 26792.9 & 1.02 & 0.02 & 1.66 \\
\hline Depth + Salinity + Area + OpenLevee + HuntLevee + Island + Breached & 12 & 26769.0 & 26793.1 & 1.23 & 0.01 & 1.85 \\
\hline \multicolumn{7}{|l|}{ Depth + Salinity + Area + OpenLevee + HuntLevee + Island + Breached +} \\
\hline DepthSD & 13 & 26767.2 & 26793.3 & 1.45 & 0.01 & 2.07 \\
\hline Depth + Salinity + OpenLevee + HuntLevee + Island + Breached & 11 & 26771.4 & 26793.5 & 1.62 & 0.01 & 2.24 \\
\hline Depth + Salinity + HuntLevee + Island + Breached + DepthSD & 11 & 26771.4 & 26793.5 & 1.62 & 0.01 & 2.24 \\
\hline Depth + Salinity + Area + OpenLevee + Island + Breached + DepthSD & 12 & 26769.4 & 26793.5 & 1.63 & 0.01 & 2.26 \\
\hline Depth + Salinity + OpenLevee + HuntLevee + Island + Breached + DepthSD & 12 & 26769.4 & 26793.5 & 1.63 & 0.01 & 2.26 \\
\hline Depth + BayDist + OpenLevee + HuntLevee + Island + Breached + DepthSD & 12 & 26769.4 & 26793.5 & 1.63 & 0.01 & 2.26 \\
\hline \multicolumn{7}{|l|}{ Depth + BayDist + Area + OpenLevee + HuntLevee + Island + Breached +} \\
\hline DepthSD & 13 & 26767.4 & 26793.5 & 1.65 & 0.01 & 2.28 \\
\hline Depth + Salinity + OpenLevee + Island + Breached & 10 & 26773.6 & 26793.7 & 1.80 & 0.01 & 2.46 \\
\hline Depth + BayDist + Island + Breached + DepthSD & 10 & 26773.6 & 26793.7 & 1.80 & 0.01 & 2.46 \\
\hline Depth + Salinity + OpenLevee + Island + Breached + DepthSD & 11 & 26771.6 & 26793.7 & 1.82 & 0.01 & 2.48 \\
\hline Depth + BayDist + OpenLevee + Island + Breached + DepthSD & 11 & 26771.6 & 26793.7 & 1.82 & 0.01 & 2.48 \\
\hline Depth + BayDist + HuntLevee + Island + Breached + DepthSD & 11 & 26771.6 & 26793.7 & 1.82 & 0.01 & 2.48 \\
\hline Depth + BayDist + Area + OpenLevee + Island + Breached + DepthSD & 12 & 26769.6 & 26793.7 & 1.83 & 0.01 & 2.50 \\
\hline Depth + Salinity + BayDist + Area + OpenLevee + HuntLevee + Island + Breached & 13 & 26767.6 & 26793.7 & 1.85 & 0.01 & 2.52 \\
\hline Depth + Salinity + Area + Island + Breached + DepthSD & 11 & 26771.8 & 26793.9 & 2.02 & 0.01 & 2.74 \\
\hline Depth + UrbanDist + Area + OpenLevee + Island + Breached & 11 & 26771.8 & 26793.9 & 2.02 & 0.01 & 2.74 \\
\hline Depth + UrbanDist + OpenLevee + HuntLevee + Island + Breached & 11 & 26771.8 & 26793.9 & 2.02 & 0.01 & 2.74 \\
\hline Depth + Salinity + Area + HuntLevee + Island + Breached + DepthSD & 12 & 26769.8 & 26793.9 & 2.03 & 0.01 & 2.76 \\
\hline Depth + BayDist + UrbanDist + OpenLevee + HuntLevee + Island + Breached & 12 & 26769.8 & 26793.9 & 2.03 & 0.01 & 2.76 \\
\hline Depth + UrbanDist + Area + OpenLevee + HuntLevee + Island + Breached & 12 & 26769.8 & 26793.9 & 2.03 & 0.01 & 2.76 \\
\hline Depth + UrbanDist + OpenLevee + Island + Breached & 10 & 26774.0 & 26794.1 & 2.20 & 0.01 & 3.00 \\
\hline
\end{tabular}




\begin{tabular}{|c|c|c|c|c|c|c|}
\hline Model name & k & $-2 \log L$ & AICc & $\triangle \mathrm{AICc}$ & $\mathbf{w}_{\mathrm{i}}$ & $\begin{array}{c}\begin{array}{c}\text { Evidence } \\
\text { ratio }\end{array} \\
\end{array}$ \\
\hline Depth + BayDist + UrbanDist + OpenLevee + Island + Breached & 11 & 26772.0 & 26794.1 & 2.22 & 0.01 & 3.03 \\
\hline Depth + BayDist + Area + Island + Breached + DepthSD & 11 & 26772.0 & 26794.1 & 2.22 & 0.01 & 3.03 \\
\hline Depth + UrbanDist + HuntLevee + Island + Breached + DepthSD & 11 & 26772.0 & 26794.1 & 2.22 & 0.01 & 3.03 \\
\hline Depth + UrbanDist + OpenLevee + HuntLevee + Island + Breached + DepthSD & 12 & 26770.0 & 26794.1 & 2.23 & 0.01 & 3.05 \\
\hline \multicolumn{7}{|l|}{ Depth + UrbanDist + Area + OpenLevee + HuntLevee + Island + Breached +} \\
\hline DepthSD & 13 & 26768.0 & 26794.1 & 2.25 & 0.01 & 3.08 \\
\hline Depth + BayDist + Island + Breached & 9 & 26776.2 & 26794.3 & 2.39 & 0.01 & 3.30 \\
\hline Depth + UrbanDist + OpenLevee + Island + Breached + DepthSD & 11 & 26772.2 & 26794.3 & 2.42 & 0.01 & 3.35 \\
\hline Depth + BayDist + Area + HuntLevee + Island + Breached + DepthSD & 12 & 26770.2 & 26794.3 & 2.43 & 0.01 & 3.37 \\
\hline Depth + UrbanDist + Area + OpenLevee + Island + Breached + DepthSD & 12 & 26770.2 & 26794.3 & 2.43 & 0.01 & 3.37 \\
\hline \multicolumn{7}{|l|}{ Depth + BayDist + UrbanDist + Area + OpenLevee + HuntLevee + Island +} \\
\hline Breached & 13 & 26768.2 & 26794.3 & 2.45 & 0.01 & 3.40 \\
\hline Depth + BayDist + UrbanDist + Area + OpenLevee + Island + Breached & 12 & 26770.4 & 26794.5 & 2.63 & 0.01 & 3.73 \\
\hline Depth + UrbanDist + Area + Island + Breached + DepthSD & 11 & 26772.6 & 26794.7 & 2.82 & 0.01 & 4.09 \\
\hline \multicolumn{7}{|l|}{ Depth + Salinity + BayDist + OpenLevee + HuntLevee + Island + Breached +} \\
\hline DepthSD & 13 & 26768.6 & 26794.7 & 2.85 & 0.01 & 4.16 \\
\hline \multicolumn{7}{|l|}{ Depth + Salinity + BayDist + Area + OpenLevee + HuntLevee + Island + Breached } \\
\hline + DepthSD & 14 & 26766.6 & 26794.8 & 2.87 & 0.01 & 4.20 \\
\hline Depth + BayDist + Area + Island + Breached & 10 & 26774.8 & 26794.9 & 3.00 & 0.01 & 4.48 \\
\hline Depth + Salinity + BayDist + OpenLevee + Island + Breached + DepthSD & 12 & 26770.8 & 26794.9 & 3.03 & 0.01 & 4.55 \\
\hline Depth + Salinity + BayDist + HuntLevee + Island + Breached + DepthSD & 12 & 26770.8 & 26794.9 & 3.03 & 0.01 & 4.55 \\
\hline Depth + UrbanDist + Area + HuntLevee + Island + Breached + DepthSD & 12 & 26770.8 & 26794.9 & 3.03 & 0.01 & 4.55 \\
\hline Depth + Salinity + BayDist + Area + OpenLevee + Island + Breached + DepthSD & 13 & 26768.8 & 26794.9 & 3.05 & 0.01 & 4.60 \\
\hline \multicolumn{7}{|l|}{ Depth + Salinity + UrbanDist + OpenLevee + HuntLevee + Island + Breached +} \\
\hline DepthSD & 13 & 26768.8 & 26794.9 & 3.05 & 0.01 & 4.60 \\
\hline \multicolumn{7}{|l|}{ Depth + BayDist + UrbanDist + OpenLevee + HuntLevee + Island + Breached +} \\
\hline DepthSD & 13 & 26768.8 & 26794.9 & 3.05 & 0.01 & 4.60 \\
\hline Depth + Area + Island + Breached & 9 & 26777.0 & 26795.1 & 3.19 & 0.01 & 4.92 \\
\hline Depth + HuntLevee + Island + Breached & 9 & 26777.0 & 26795.1 & 3.19 & 0.01 & 4.92 \\
\hline Depth + Salinity + UrbanDist + Island + Breached + DepthSD & 11 & 26773.0 & 26795.1 & 3.22 & 0.01 & 4.99 \\
\hline Depth + BayDist + UrbanDist + Island + Breached + DepthSD & 11 & 26773.0 & 26795.1 & 3.22 & 0.01 & 4.99 \\
\hline Depth + BayDist + Area + HuntLevee + Island + Breached & 11 & 26773.0 & 26795.1 & 3.22 & 0.01 & 4.99 \\
\hline Depth + Salinity + UrbanDist + OpenLevee + HuntLevee + Island + Breached & 12 & 26771.0 & 26795.1 & 3.23 & 0.01 & 5.03 \\
\hline Depth + Salinity + UrbanDist + HuntLevee + Island + Breached + DepthSD & 12 & 26771.0 & 26795.1 & 3.23 & 0.01 & 5.03 \\
\hline Depth + BayDist + UrbanDist + HuntLevee + Island + Breached + DepthSD & 12 & 26771.0 & 26795.1 & 3.23 & 0.01 & 5.03 \\
\hline \multicolumn{7}{|l|}{ Depth + Salinity + UrbanDist + Area + OpenLevee + HuntLevee + Island +} \\
\hline Breached & 13 & 26769.0 & 26795.1 & 3.25 & 0.01 & 5.08 \\
\hline \multicolumn{7}{|l|}{ Depth + Salinity + UrbanDist + Area + OpenLevee + HuntLevee + Island +} \\
\hline Breached + DepthSD & 14 & 26767.0 & 26795.2 & 3.27 & 0.01 & 5.13 \\
\hline Depth + Salinity + UrbanDist + OpenLevee + Island + Breached & 11 & 26773.2 & 26795.3 & 3.42 & 0.00 & 5.52 \\
\hline Depth + Salinity + BayDist + Area + Island + Breached + DepthSD & 12 & 26771.2 & 26795.3 & 3.43 & 0.00 & 5.56 \\
\hline Depth + Salinity + UrbanDist + Area + OpenLevee + Island + Breached & 12 & 26771.2 & 26795.3 & 3.43 & 0.00 & 5.56 \\
\hline Depth + Salinity + UrbanDist + OpenLevee + Island + Breached + DepthSD & 12 & 26771.2 & 26795.3 & 3.43 & 0.00 & 5.56 \\
\hline Depth + BayDist + UrbanDist + OpenLevee + Island + Breached + DepthSD & 12 & 26771.2 & 26795.3 & 3.43 & 0.00 & 5.56 \\
\hline \multicolumn{7}{|l|}{ Depth + Salinity + BayDist + UrbanDist + OpenLevee + HuntLevee + Island +} \\
\hline Breached & 13 & 26769.2 & 26795.3 & 3.45 & 0.00 & 5.61 \\
\hline Depth + Salinity + BayDist + Area + HuntLevee + Island + Breached + DepthSD & 13 & 26769.2 & 26795.3 & 3.45 & 0.00 & 5.61 \\
\hline
\end{tabular}




\begin{tabular}{|c|c|c|c|c|c|c|}
\hline Model name & k & $-2 \log L$ & AICc & $\triangle \mathrm{AICc}$ & $w_{i}$ & $\begin{array}{c}\text { Evidence } \\
\text { ratio }\end{array}$ \\
\hline \multicolumn{7}{|l|}{ Depth + BayDist + UrbanDist + Area + OpenLevee + HuntLevee + Island +} \\
\hline Breached + DepthSD & 14 & 26767.2 & 26795.4 & 3.47 & 0.00 & 5.67 \\
\hline Depth + Area + HuntLevee + Island + Breached & 10 & 26775.4 & 26795.5 & 3.60 & 0.00 & 6.05 \\
\hline Depth + Salinity + BayDist + UrbanDist + OpenLevee + Island + Breached & 12 & 26771.4 & 26795.5 & 3.63 & 0.00 & 6.15 \\
\hline \multicolumn{7}{|l|}{ Depth + Salinity + UrbanDist + Area + OpenLevee + Island + Breached +} \\
\hline DepthSD & 13 & 26769.4 & 26795.5 & 3.65 & 0.00 & 6.20 \\
\hline Depth + Area + OpenLevee + HuntLevee + Breached + DepthSD & 11 & 26773.6 & 26795.7 & 3.82 & 0.00 & 6.74 \\
\hline Depth + Salinity + UrbanDist + Area + HuntLevee + Island + Breached + DepthSD & 13 & 26769.6 & 26795.7 & 3.85 & 0.00 & 6.86 \\
\hline \multicolumn{7}{|l|}{ Depth + BayDist + UrbanDist + Area + OpenLevee + Island + Breached +} \\
\hline DepthSD & 13 & 26769.6 & 26795.7 & 3.85 & 0.00 & 6.86 \\
\hline \multicolumn{7}{|l|}{ Depth + Salinity + BayDist + UrbanDist + Area + OpenLevee + HuntLevee +} \\
\hline Island + Breached & 14 & 26767.6 & 26795.8 & 3.87 & 0.00 & 6.92 \\
\hline
\end{tabular}


Table 8. The ranking of candidate models at the pond scale for foraging medium shorebirds in the former salt production ponds of South San Francisco Bay, California.

[Models are ranked by differences in Akaike's information criterion and only models of $\Delta$ AIC $<4$ and the null model are presented. Variables considered included the mean pond depth (Depth), the presence of islands (Island), variation in topography (DepthSD), \% of pond levee open to hunting (HuntLevee), \% of pond levee open to the public (OpenLevee), pond area (Area), water salinity, pond distance to urban areas (UrbanDist), and pond distance to the bay (BayDist). k: Number of estimated parameters in the model. AICc: Second-order Akaike's information criterion. $\triangle$ AICc: The difference between the AICc of the current model and the AICc of the top model. wi: Akaike weight-likelihood of the model relative to candidate models. Evidence ratio: The weight of evidence that the current model is inferior to the top model]

\begin{tabular}{|c|c|c|c|c|c|c|}
\hline Model name & k & $-2 \log L$ & AlCc & $\triangle \mathrm{AICc}$ & $w_{\mathrm{i}}$ & $\begin{array}{c}\text { Evidence } \\
\text { ratio }\end{array}$ \\
\hline \multicolumn{7}{|l|}{ Depth + Salinity + BayDist + HuntLevee + Island + Breached + } \\
\hline DepthSD & 12 & 19571.9 & 19596.1 & 0.00 & 0.05 & 1.00 \\
\hline Depth + BayDist + HuntLevee + Island + Breached + DepthSD & 11 & 19574.1 & 19596.2 & 0.12 & 0.05 & 1.06 \\
\hline \multicolumn{7}{|l|}{ Depth + Salinity + BayDist + OpenLevee + HuntLevee + Island +} \\
\hline Breached + DepthSD & 13 & 19571.1 & 19597.2 & 1.14 & 0.03 & 1.77 \\
\hline \multicolumn{7}{|l|}{ Depth + BayDist + OpenLevee + HuntLevee + Island + Breached +} \\
\hline DepthSD & 12 & 19573.2 & 19597.3 & 1.26 & 0.03 & 1.88 \\
\hline \multicolumn{7}{|l|}{ Depth + Salinity + BayDist + UrbanDist + HuntLevee + Island +} \\
\hline Breached + DepthSD & 13 & 19571.4 & 19597.6 & 1.50 & 0.02 & 2.12 \\
\hline \multicolumn{7}{|l|}{ Depth + BayDist + UrbanDist + HuntLevee + Island + Breached +} \\
\hline DepthSD & 12 & 19573.4 & 19597.6 & 1.50 & 0.02 & 2.12 \\
\hline Depth + BayDist + OpenLevee + HuntLevee + Island + Breached & 11 & 19575.5 & 19597.6 & 1.52 & 0.02 & 2.14 \\
\hline \multicolumn{7}{|l|}{ Depth + Salinity + BayDist + OpenLevee + HuntLevee + Island +} \\
\hline Breached & 12 & 19573.5 & 19597.6 & 1.56 & 0.02 & 2.18 \\
\hline Depth + Salinity + HuntLevee + Island + Breached + DepthSD & 11 & 19575.5 & 19597.6 & 1.58 & 0.02 & 2.21 \\
\hline \multicolumn{7}{|l|}{ Depth + Salinity + BayDist + Area + HuntLevee + Island + Breached +} \\
\hline DepthSD & 13 & 19571.9 & 19598.0 & 1.94 & 0.02 & 2.64 \\
\hline Depth + BayDist + Area + HuntLevee + Island + Breached + DepthSD & 12 & 19574.0 & 19598.1 & 2.04 & 0.02 & 2.77 \\
\hline Depth + HuntLevee + Island + Breached + DepthSD & 10 & 19578.0 & 19598.1 & 2.07 & 0.02 & 2.81 \\
\hline Depth + Salinity + BayDist + Island + Breached + DepthSD & 11 & 19576.3 & 19598.4 & 2.30 & 0.02 & 3.16 \\
\hline Depth + BayDist + HuntLevee + Island + Breached & 10 & 19578.3 & 19598.4 & 2.37 & 0.02 & 3.27 \\
\hline \multicolumn{7}{|l|}{ Depth + Salinity + OpenLevee + HuntLevee + Island + Breached +} \\
\hline DepthSD & 12 & 19574.4 & 19598.6 & 2.50 & 0.01 & 3.49 \\
\hline Depth + Salinity + BayDist + HuntLevee + Island + Breached & 11 & 19576.5 & 19598.6 & 2.52 & 0.01 & 3.53 \\
\hline \multicolumn{7}{|l|}{ Depth + Salinity + BayDist + UrbanDist + OpenLevee + HuntLevee +} \\
\hline Island + Breached + DepthSD & 14 & 19570.5 & 19598.6 & 2.58 & 0.01 & 3.63 \\
\hline \multicolumn{7}{|l|}{ Depth + BayDist + UrbanDist + OpenLevee + HuntLevee + Island +} \\
\hline Breached + DepthSD & 13 & 19572.5 & 19598.6 & 2.58 & 0.01 & 3.63 \\
\hline Depth + BayDist + Island + Breached + DepthSD & 10 & 19578.6 & 19598.7 & 2.61 & 0.01 & 3.68 \\
\hline \multicolumn{7}{|l|}{ Depth + Salinity + UrbanDist + HuntLevee + Island + Breached +} \\
\hline DepthSD & 12 & 19574.7 & 19598.9 & 2.80 & 0.01 & 4.06 \\
\hline \multicolumn{7}{|l|}{ Depth + BayDist + UrbanDist + OpenLevee + HuntLevee + Island +} \\
\hline Breached & 12 & 19574.8 & 19598.9 & 2.84 & 0.01 & 4.14 \\
\hline \multicolumn{7}{|l|}{ Depth + Salinity + BayDist + UrbanDist + OpenLevee + HuntLevee +} \\
\hline Island + Breached & 13 & 19572.9 & 19599.0 & 2.98 & 0.01 & 4.43 \\
\hline Depth + OpenLevee + HuntLevee + Island + Breached + DepthSD & 11 & 19577.0 & 19599.1 & 3.00 & 0.01 & 4.49 \\
\hline
\end{tabular}




\begin{tabular}{|c|c|c|c|c|c|c|}
\hline Model name & k & $-2 \log L$ & AICc & $\triangle \mathrm{AICc}$ & $\mathbf{w}_{\mathrm{i}}$ & $\begin{array}{c}\text { Evidence } \\
\text { ratio }\end{array}$ \\
\hline \multicolumn{7}{|l|}{ Depth + Salinity + BayDist + Area + OpenLevee + HuntLevee + Island +} \\
\hline Breached + DepthSD & 14 & 19571.0 & 19599.2 & 3.12 & 0.01 & 4.75 \\
\hline \multicolumn{7}{|l|}{ Depth + BayDist + Area + OpenLevee + HuntLevee + Island + Breached } \\
\hline + DepthSD & 13 & 19573.2 & 19599.3 & 3.24 & 0.01 & 5.05 \\
\hline Depth + BayDist + HuntLevee + Breached + DepthSD & 10 & 19579.4 & 19599.5 & 3.43 & 0.01 & 5.55 \\
\hline \multicolumn{7}{|l|}{ Depth + BayDist + UrbanDist + Area + HuntLevee + Island + Breached } \\
\hline + DepthSD & 13 & 19573.4 & 19599.6 & 3.50 & 0.01 & 5.75 \\
\hline \multicolumn{7}{|l|}{ Depth + Salinity + BayDist + UrbanDist + Area + HuntLevee + Island +} \\
\hline Breached + DepthSD & 14 & 19571.4 & 19599.6 & 3.52 & 0.01 & 5.81 \\
\hline Depth + BayDist + Area + OpenLevee + HuntLevee + Island + Breached & 12 & 19575.5 & 19599.6 & 3.54 & 0.01 & 5.87 \\
\hline \multicolumn{7}{|l|}{ Depth + Salinity + BayDist + Area + OpenLevee + HuntLevee + Island +} \\
\hline Breached & 13 & 19573.5 & 19599.6 & 3.58 & 0.01 & 5.98 \\
\hline Depth + Salinity + Area + HuntLevee + Island + Breached + DepthSD & 12 & 19575.5 & 19599.6 & 3.58 & 0.01 & 5.99 \\
\hline \multicolumn{7}{|l|}{ Depth + Salinity + BayDist + OpenLevee + Island + Breached +} \\
\hline DepthSD & 12 & 19575.6 & 19599.8 & 3.70 & 0.01 & 6.36 \\
\hline Depth + Salinity + BayDist + UrbanDist + Island + Breached + DepthSD & 12 & 19575.7 & 19599.8 & 3.72 & 0.01 & 6.42 \\
\hline Depth + BayDist + UrbanDist + HuntLevee + Island + Breached & 11 & 19577.8 & 19599.9 & 3.80 & 0.01 & 6.70 \\
\hline Depth + BayDist + UrbanDist + Island + Breached + DepthSD & 11 & 19577.8 & 19599.9 & 3.88 & 0.01 & 6.97 \\
\hline Depth + Salinity + Island + Breached + DepthSD & 10 & 19579.9 & 19599.9 & 3.89 & 0.01 & 6.99 \\
\hline Depth + BayDist + HuntLevee + Island + DepthSD & 10 & 19579.9 & 19600.0 & 3.95 & 0.01 & 7.20 \\
\hline \multicolumn{7}{|l|}{ Depth + UrbanDist + OpenLevee + HuntLevee + Island + Breached +} \\
\hline DepthSD & 12 & 19575.9 & 19600.0 & 3.98 & 0.01 & 7.32 \\
\hline Null Model & 5 & 19720.5 & 19730.5 & 134.45 & $3.16 \mathrm{E}-31$ & $1.57 \mathrm{E}+29$ \\
\hline
\end{tabular}


Table 9. The ranking of candidate models at the pond scale for roosting medium shorebirds in the former salt production ponds of South San Francisco Bay, California

[Models are ranked by differences in Akaike's information criterion and only models of $\triangle \mathrm{AIC}<4$ and the null model are presented. Birds were surveyed across the former salt ponds during October through April 2002-2015. Variables considered included the mean pond depth (Depth), the presence of islands (Island), variation in topography (DepthSD), \% of pond levee open to hunting (HuntLevee), \% of pond levee open to the public (OpenLevee), pond area (Area), water salinity, pond distance to urban areas (UrbanDist), and pond distance to the bay (BayDist). k: Number of estimated parameters in the model. AICc: Second-order Akaike's information criterion. $\triangle$ AICc: The difference between the AICc of the current model and the AICc of the top model. wi: Akaike weight-likelihood of the model relative to candidate models. Evidence ratio: The weight of evidence that the current model is inferior to the top model]

\begin{tabular}{|c|c|c|c|c|c|c|}
\hline Model name & k & $-2 \log L$ & AICc & $\triangle \mathrm{AICc}$ & $\mathbf{w}_{\mathrm{i}}$ & $\begin{array}{c}\text { Evidence } \\
\text { ratio }\end{array}$ \\
\hline Depth + Salinity + HuntLevee + Island + Breached + DepthSD & 11 & 26039.2 & 26061.3 & 0.00 & 0.10 & 1.00 \\
\hline Depth + HuntLevee + Island + Breached + DepthSD & 10 & 26042.0 & 26062.1 & 0.78 & 0.07 & 1.48 \\
\hline Depth + Salinity + UrbanDist + HuntLevee + Island + Breached + DepthSD & 12 & 26038.4 & 26062.5 & 1.22 & 0.05 & 1.84 \\
\hline Depth + Salinity + OpenLevee + HuntLevee + Island + Breached + DepthSD & 12 & 26038.8 & 26063.0 & 1.62 & 0.04 & 2.24 \\
\hline Depth + UrbanDist + HuntLevee + Island + Breached + DepthSD & 11 & 26041.0 & 26063.1 & 1.80 & 0.04 & 2.46 \\
\hline Depth + Salinity + BayDist + HuntLevee + Island + Breached + DepthSD & 12 & 26039.0 & 26063.1 & 1.82 & 0.04 & 2.48 \\
\hline Depth + Salinity + PondArea + HuntLevee + Island + Breached + DepthSD & 12 & 26039.0 & 26063.1 & 1.82 & 0.04 & 2.48 \\
\hline Depth + OpenLevee + HuntLevee + Island + Breached + DepthSD & 11 & 26041.6 & 26063.7 & 2.40 & 0.03 & 3.32 \\
\hline Depth + BayDist + HuntLevee + Island + Breached + DepthSD & 11 & 26041.8 & 26063.9 & 2.60 & 0.03 & 3.67 \\
\hline Depth + PondArea + HuntLevee + Island + Breached + DepthSD & 11 & 26041.8 & 26063.9 & 2.60 & 0.03 & 3.67 \\
\hline Depth + Salinity + HuntLevee + Island + DepthSD & 10 & 26044.2 & 26064.3 & 2.98 & 0.02 & 4.45 \\
\hline \multicolumn{7}{|l|}{ Depth + Salinity + UrbanDist + OpenLevee + HuntLevee + Island + Breached } \\
\hline+ DepthSD & 13 & 26038.2 & 26064.3 & 3.03 & 0.02 & 4.56 \\
\hline \multicolumn{7}{|l|}{ Depth + Salinity + UrbanDist + PondArea + HuntLevee + Island + Breached +} \\
\hline DepthSD & 13 & 26038.4 & 26064.5 & 3.23 & 0.02 & 5.04 \\
\hline \multicolumn{7}{|l|}{ Depth + Salinity + PondArea + OpenLevee + HuntLevee + Island + Breached +} \\
\hline DepthSD & 13 & 26038.4 & 26064.5 & 3.23 & 0.02 & 5.04 \\
\hline Depth + UrbanDist + OpenLevee + HuntLevee + Island + Breached + DepthSD & 12 & 26040.6 & 26064.7 & 3.42 & 0.02 & 5.52 \\
\hline \multicolumn{7}{|l|}{ Depth + Salinity + BayDist + OpenLevee + HuntLevee + Island + Breached +} \\
\hline DepthSD & 13 & 26038.6 & 26064.7 & 3.43 & 0.02 & 5.57 \\
\hline \multicolumn{7}{|l|}{ Depth + Salinity + BayDist + PondArea + HuntLevee + Island + Breached +} \\
\hline DepthSD & 13 & 26038.8 & 26064.9 & 3.63 & 0.02 & 6.16 \\
\hline Depth + UrbanDist + PondArea + HuntLevee + Island + Breached + DepthSD & 12 & 26041.0 & 26065.1 & 3.82 & 0.01 & 6.74 \\
\hline Depth + Island + Breached + DepthSD & 9 & 26047.2 & 26065.3 & 3.97 & 0.01 & 7.28 \\
\hline Null Model & 5 & 26158.6 & 26168.6 & 107.33 & $4.84 \mathrm{E}-25$ & $2.02 \mathrm{E}+23$ \\
\hline
\end{tabular}


Table 10. The ranking of candidate models at the pond scale for foraging wadersa in the former salt production ponds of South San Francisco Bay, California.

[Models are ranked by differences in Akaike's information criterion and only models of $\triangle \mathrm{AIC}<4$ and the null model are presented. Birds were surveyed across the former salt ponds during October through April 2002-2015. Variables considered included the mean pond depth (Depth), the presence of islands (Island), variation in topography (DepthSD), \% of pond levee open to hunting (HuntLevee), \% of pond levee open to the public (OpenLevee), pond area (Area), water salinity, pond distance to urban areas (UrbanDist), and pond distance to the bay (BayDist). k: Number of estimated parameters in the model. AICc: Second-order Akaike's information criterion. $\triangle$ AICc: The difference between the AICc of the current model and the AICc of the top model. wi: Akaike weight-likelihood of the model relative to candidate models. Evidence ratio: The weight of evidence that the current model is inferior to the top model]

\begin{tabular}{|c|c|c|c|c|c|c|}
\hline Model name & k & $-2 \log L$ & AICc & $\triangle \mathrm{AICc}$ & $w_{i}$ & $\begin{array}{c}\text { Evidence } \\
\text { ratio }\end{array}$ \\
\hline \multicolumn{7}{|l|}{ Depth + Salinity + UrbanDist + PondArea + OpenLevee + HuntLevee + } \\
\hline Island + Breached & 13 & 11109.9 & 11134.0 & 0.00 & 0.05 & 1.00 \\
\hline \multicolumn{7}{|l|}{ Depth + Salinity + UrbanDist + PondArea + OpenLevee + Island +} \\
\hline Breached & 12 & 11111.9 & 11136.0 & 0.02 & 0.05 & 1.01 \\
\hline Depth + Salinity + UrbanDist + PondArea + OpenLevee + Island & 11 & 11114.0 & 11136.1 & 0.09 & 0.05 & 1.04 \\
\hline \multicolumn{7}{|l|}{ Depth + Salinity + UrbanDist + PondArea + OpenLevee + HuntLevee +} \\
\hline Island & 12 & 11112.1 & 11136.3 & 0.26 & 0.04 & 1.14 \\
\hline \multicolumn{7}{|l|}{ Depth + Salinity + UrbanDist + PondArea + OpenLevee + Island +} \\
\hline Breached + DepthSD & 13 & 11110.5 & 11136.7 & 0.68 & 0.03 & 1.40 \\
\hline \multicolumn{7}{|l|}{ Depth + Salinity + UrbanDist + PondArea + OpenLevee + HuntLevee +} \\
\hline Island + Breached + DepthSD & 14 & 11108.5 & 11136.7 & 0.70 & 0.03 & 1.42 \\
\hline \multicolumn{7}{|l|}{ Depth + Salinity + UrbanDist + PondArea + HuntLevee + Island +} \\
\hline Breached + DepthSD & 13 & 11110.6 & 11136.8 & 0.76 & 0.03 & 1.46 \\
\hline \multicolumn{7}{|l|}{ Depth + Salinity + BayDist + UrbanDist + PondArea + OpenLevee +} \\
\hline Island + DepthSD & 13 & 11110.8 & 11136.9 & 0.90 & 0.03 & 1.57 \\
\hline \multicolumn{7}{|l|}{ Depth + Salinity + BayDist + UrbanDist + PondArea + OpenLevee +} \\
\hline Island + Breached + DepthSD & 14 & 11108.8 & 11136.9 & 0.94 & 0.03 & 1.60 \\
\hline \multicolumn{7}{|l|}{ Depth + Salinity + UrbanDist + PondArea + OpenLevee + Island +} \\
\hline DepthSD & 12 & 11112.8 & 11137.0 & 0.96 & 0.03 & 1.62 \\
\hline \multicolumn{7}{|l|}{ Depth + Salinity + BayDist + UrbanDist + PondArea + OpenLevee +} \\
\hline HuntLevee + Island + Breached + DepthSD & 15 & 11106.8 & 11137.0 & 1.00 & 0.03 & 1.65 \\
\hline \multicolumn{7}{|l|}{ Depth + Salinity + UrbanDist + PondArea + Island + Breached +} \\
\hline DepthSD & 12 & 11112.9 & 11137.0 & 1.00 & 0.03 & 1.65 \\
\hline \multicolumn{7}{|l|}{ Depth + Salinity + BayDist + UrbanDist + PondArea + OpenLevee +} \\
\hline HuntLevee + Island + DepthSD & 14 & 11109.0 & 11137.1 & 1.14 & 0.03 & 1.77 \\
\hline \multicolumn{7}{|l|}{ Depth + Salinity + BayDist + UrbanDist + PondArea + OpenLevee +} \\
\hline Island & 12 & 11113.0 & 11137.2 & 1.16 & 0.03 & 1.79 \\
\hline \multicolumn{7}{|l|}{ Depth + Salinity + UrbanDist + PondArea + OpenLevee + HuntLevee +} \\
\hline Island + DepthSD & 13 & 11111.0 & 11137.2 & 1.18 & 0.03 & 1.80 \\
\hline \multicolumn{7}{|l|}{ Depth + Salinity + BayDist + UrbanDist + PondArea + HuntLevee +} \\
\hline Island + Breached + DepthSD & 14 & 11109.1 & 11137.2 & 1.24 & 0.03 & 1.86 \\
\hline \multicolumn{7}{|l|}{ Depth + Salinity + BayDist + UrbanDist + PondArea + OpenLevee +} \\
\hline HuntLevee + Island & 13 & 11111.2 & 11137.3 & 1.34 & 0.02 & 1.95 \\
\hline \multicolumn{7}{|l|}{ Depth + Salinity + BayDist + UrbanDist + PondArea + OpenLevee +} \\
\hline HuntLevee + Island + Breached & 14 & 11109.2 & 11137.4 & 1.36 & 0.02 & 1.97 \\
\hline \multicolumn{7}{|l|}{ Depth + Salinity + BayDist + UrbanDist + PondArea + OpenLevee +} \\
\hline Island + Breached & 13 & 11111.2 & 11137.4 & 1.36 & 0.02 & 1.97 \\
\hline
\end{tabular}




\begin{tabular}{|c|c|c|c|c|c|c|}
\hline Model name & k & $-2 \log L$ & AICc & $\triangle \mathrm{AICc}$ & $\mathbf{w}_{\mathrm{i}}$ & $\begin{array}{c}\text { Evidence } \\
\text { ratio }\end{array}$ \\
\hline \multicolumn{7}{|l|}{ Depth + Salinity + BayDist + UrbanDist + PondArea + Island + Breached } \\
\hline + DepthSD & 13 & 11111.3 & 11137.5 & 1.48 & 0.02 & 2.10 \\
\hline Depth + Salinity + PondArea + OpenLevee + HuntLevee + Island & 11 & 11116.2 & 11138.3 & 2.29 & 0.02 & 3.13 \\
\hline Depth + Salinity + PondArea + OpenLevee + Island & 10 & 11118.2 & 11138.3 & 2.29 & 0.02 & 3.14 \\
\hline \multicolumn{7}{|l|}{ Depth + Salinity + BayDist + PondArea + OpenLevee + Island +} \\
\hline DepthSD & 12 & 11114.2 & 11138.4 & 2.36 & 0.01 & 3.26 \\
\hline \multicolumn{7}{|l|}{ Depth + Salinity + UrbanDist + PondArea + HuntLevee + Island +} \\
\hline Breached & 12 & 11114.3 & 11138.4 & 2.38 & 0.01 & 3.29 \\
\hline \multicolumn{7}{|l|}{ Depth + Salinity + BayDist + PondArea + OpenLevee + HuntLevee +} \\
\hline Island + DepthSD & 13 & 11112.3 & 11138.4 & 2.42 & 0.01 & 3.35 \\
\hline \multicolumn{7}{|l|}{ Depth + Salinity + PondArea + OpenLevee + HuntLevee + Island +} \\
\hline Breached & 12 & 11114.3 & 11138.4 & 2.42 & 0.01 & 3.36 \\
\hline Depth + Salinity + BayDist + PondArea + OpenLevee + Island & 11 & 11116.4 & 11138.5 & 2.53 & 0.01 & 3.53 \\
\hline \multicolumn{7}{|l|}{ Depth + Salinity + BayDist + PondArea + OpenLevee + HuntLevee +} \\
\hline Island & 12 & 11114.4 & 11138.5 & 2.54 & 0.01 & 3.56 \\
\hline Depth + Salinity + PondArea + OpenLevee + Island + Breached & 11 & 11116.5 & 11138.6 & 2.63 & 0.01 & 3.72 \\
\hline \multicolumn{7}{|l|}{ Depth + Salinity + BayDist + PondArea + OpenLevee + HuntLevee +} \\
\hline Island + Breached + DepthSD & 14 & 11110.6 & 11138.7 & 2.74 & 0.01 & 3.93 \\
\hline \multicolumn{7}{|l|}{ Depth + Salinity + UrbanDist + PondArea + HuntLevee + Island +} \\
\hline DepthSD & 12 & 11114.6 & 11138.7 & 2.74 & 0.01 & 3.94 \\
\hline Depth + Salinity + UrbanDist + PondArea + Island + DepthSD & 11 & 11116.7 & 11138.8 & 2.77 & 0.01 & 3.98 \\
\hline \multicolumn{7}{|l|}{ Depth + Salinity + BayDist + UrbanDist + PondArea + HuntLevee +} \\
\hline Island + DepthSD & 13 & 11112.7 & 11138.9 & 2.86 & 0.01 & 4.18 \\
\hline \multicolumn{7}{|l|}{ Depth + Salinity + BayDist + PondArea + OpenLevee + Island +} \\
\hline Breached + DepthSD & 13 & 11112.7 & 11138.9 & 2.86 & 0.01 & 4.18 \\
\hline Depth + Salinity + BayDist + UrbanDist + PondArea + Island + DepthSD & 12 & 11114.7 & 11138.9 & 2.86 & 0.01 & 4.18 \\
\hline Depth + Salinity + UrbanDist + PondArea + Island + Breached & 11 & 11116.8 & 11138.9 & 2.91 & 0.01 & 4.27 \\
\hline \multicolumn{7}{|l|}{ Depth + Salinity + BayDist + PondArea + OpenLevee + HuntLevee +} \\
\hline Island + Breached & 13 & 11112.8 & 11139.0 & 2.98 & 0.01 & 4.44 \\
\hline \multicolumn{7}{|l|}{ Depth + Salinity + BayDist + PondArea + OpenLevee + Island +} \\
\hline Breached & 12 & 11115.0 & 11139.2 & 3.16 & 0.01 & 4.86 \\
\hline \multicolumn{7}{|l|}{ Depth + Salinity + PondArea + OpenLevee + HuntLevee + Island +} \\
\hline Breached + DepthSD & 13 & 11113.3 & 11139.5 & 3.48 & 0.01 & 5.70 \\
\hline Depth + Salinity + PondArea + OpenLevee + Island + DepthSD & 11 & 11117.4 & 11139.5 & 3.49 & 0.01 & 5.71 \\
\hline \multicolumn{7}{|l|}{ Depth + Salinity + PondArea + OpenLevee + HuntLevee + Island +} \\
\hline DepthSD & 12 & 11115.4 & 11139.5 & 3.50 & 0.01 & 5.76 \\
\hline \multicolumn{7}{|l|}{ Depth + Salinity + PondArea + OpenLevee + Island + Breached +} \\
\hline DepthSD & 12 & 11115.6 & 11139.7 & 3.68 & 0.01 & 6.30 \\
\hline \multicolumn{7}{|l|}{ Depth + Salinity + BayDist + PondArea + HuntLevee + Island +} \\
\hline Breached + DepthSD & 13 & 11113.7 & 11139.8 & 3.80 & 0.01 & 6.69 \\
\hline Null Model & 5 & 11424.0 & 11434.1 & 298.07 & $8.96 \mathrm{E}-67$ & $5.32 \mathrm{E}+64$ \\
\hline
\end{tabular}


Table 11. The ranking of candidate models at the pond scale for roosting waders in the former salt production ponds of South San Francisco Bay, California.

[Models are ranked by differences in Akaike's information criterion and only models of $\triangle \mathrm{AIC}<4$ and the null model are presented. Birds were surveyed across the former salt ponds during October through April 2002-2015. Variables considered included the mean pond depth (Depth), the presence of islands (Island), variation in topography (DepthSD), \% of pond levee open to hunting (HuntLevee), \% of pond levee open to the public (OpenLevee), pond area (Area), water salinity, pond distance to urban areas (UrbanDist), and pond distance to the bay (BayDist). k: Number of estimated parameters in the model. AICc: Second-order Akaike's information criterion. $\triangle$ AICc: The difference between the AICc of the current model and the AICc of the top model. wi: Akaike weight-likelihood of the model relative to candidate models. Evidence ratio: The weight of evidence that the current model is inferior to the top model]

\begin{tabular}{|c|c|c|c|c|c|c|}
\hline Model name & k & $-2 \log L$ & AICc & $\triangle \mathrm{AICc}$ & $w_{i}$ & $\begin{array}{l}\text { Evidence } \\
\text { ratio }\end{array}$ \\
\hline Salinity + UrbanDist + PondArea + HuntLevee + Island & 10 & 11390.3 & 11410.4 & 0.00 & 0.09 & 1.00 \\
\hline Salinity + BayDist + UrbanDist + PondArea + HuntLevee + Island & 11 & 11388.7 & 11410.8 & 0.36 & 0.08 & 1.19 \\
\hline \multicolumn{7}{|l|}{ Salinity + BayDist + UrbanDist + PondArea + HuntLevee + Island +} \\
\hline DepthSD & 12 & 11387.3 & 11411.4 & 1.01 & 0.06 & 1.66 \\
\hline Salinity + UrbanDist + PondArea + OpenLevee + HuntLevee + Island & 11 & 11389.8 & 11411.9 & 1.48 & 0.04 & 2.09 \\
\hline Salinity + UrbanDist + PondArea + HuntLevee + Island + DepthSD & 11 & 11390.0 & 11412.1 & 1.70 & 0.04 & 2.33 \\
\hline Depth + Salinity + UrbanDist + PondArea + HuntLevee + Island & 11 & 11390.3 & 11412.4 & 1.94 & 0.03 & 2.63 \\
\hline Salinity + UrbanDist + PondArea + HuntLevee + Island + Breached & 11 & 11390.3 & 11412.4 & 1.94 & 0.03 & 2.63 \\
\hline \multicolumn{7}{|l|}{ Depth + Salinity + BayDist + UrbanDist + PondArea + HuntLevee +} \\
\hline Island & 12 & 11388.3 & 11412.4 & 2.01 & 0.03 & 2.73 \\
\hline \multicolumn{7}{|l|}{ Salinity + BayDist + UrbanDist + PondArea + OpenLevee + HuntLevee +} \\
\hline Island + DepthSD & 13 & 11386.3 & 11412.5 & 2.05 & 0.03 & 2.79 \\
\hline \multicolumn{7}{|l|}{ Salinity + BayDist + UrbanDist + PondArea + OpenLevee + HuntLevee +} \\
\hline Island & 12 & 11388.4 & 11412.5 & 2.09 & 0.03 & 2.85 \\
\hline \multicolumn{7}{|l|}{ Salinity + BayDist + UrbanDist + PondArea + HuntLevee + Island +} \\
\hline Breached & 12 & 11388.5 & 11412.6 & 2.19 & 0.03 & 2.99 \\
\hline Salinity + BayDist + PondArea + HuntLevee + Island & 10 & 11392.9 & 11413.0 & 2.60 & 0.03 & 3.67 \\
\hline \multicolumn{7}{|l|}{ Salinity + UrbanDist + PondArea + OpenLevee + HuntLevee + Island +} \\
\hline DepthSD & 12 & 11389.0 & 11413.1 & 2.69 & 0.02 & 3.84 \\
\hline \multicolumn{7}{|l|}{ Salinity + BayDist + UrbanDist + PondArea + HuntLevee + Island +} \\
\hline Breached + DepthSD & 13 & 11387.1 & 11413.3 & 2.83 & 0.02 & 4.12 \\
\hline \multicolumn{7}{|l|}{ Depth + Salinity + BayDist + UrbanDist + PondArea + HuntLevee +} \\
\hline Island + DepthSD & 13 & 11387.2 & 11413.3 & 2.87 & 0.02 & 4.20 \\
\hline Salinity + PondArea + HuntLevee + Island & 9 & 11395.4 & 11413.4 & 3.01 & 0.02 & 4.50 \\
\hline Salinity + BayDist + PondArea + HuntLevee + Island + DepthSD & 11 & 11391.4 & 11413.5 & 3.04 & 0.02 & 4.56 \\
\hline \multicolumn{7}{|l|}{ Depth + Salinity + UrbanDist + PondArea + OpenLevee + HuntLevee +} \\
\hline Island & 12 & 11389.7 & 11413.8 & 3.41 & 0.02 & 5.51 \\
\hline \multicolumn{7}{|l|}{ Salinity + UrbanDist + PondArea + OpenLevee + HuntLevee + Island +} \\
\hline Breached & 12 & 11389.8 & 11413.9 & 3.49 & 0.02 & 5.73 \\
\hline \multicolumn{7}{|l|}{ Salinity + UrbanDist + PondArea + HuntLevee + Island + Breached +} \\
\hline DepthSD & 12 & 11390.0 & 11414.1 & 3.65 & 0.01 & 6.21 \\
\hline \multicolumn{7}{|l|}{ Depth + Salinity + UrbanDist + PondArea + HuntLevee + Island +} \\
\hline DepthSD & 12 & 11390.0 & 11414.1 & 3.69 & 0.01 & 6.33 \\
\hline \multicolumn{7}{|l|}{ Depth + Salinity + BayDist + UrbanDist + PondArea + OpenLevee +} \\
\hline HuntLevee + Island & 13 & 11388.0 & 11414.2 & 3.77 & 0.01 & 6.59 \\
\hline \multicolumn{7}{|l|}{ Depth + Salinity + UrbanDist + PondArea + HuntLevee + Island +} \\
\hline Breached & 12 & 11390.2 & 11414.3 & 3.91 & 0.01 & 7.07 \\
\hline
\end{tabular}




\begin{tabular}{|c|c|c|c|c|c|c|}
\hline Model name & k & $-2 \log L$ & AlCc & $\triangle \mathrm{AICc}$ & $w_{i}$ & $\begin{array}{c}\text { Evidence } \\
\text { ratio }\end{array}$ \\
\hline \multicolumn{7}{|c|}{ Depth + Salinity + BayDist + UrbanDist + PondArea + HuntLevee +} \\
\hline Island + Breached & 13 & 11388.3 & 11414.4 & 3.97 & 0.01 & 7.28 \\
\hline \multicolumn{7}{|c|}{ Depth + Salinity + BayDist + UrbanDist + PondArea + OpenLevee +} \\
\hline HuntLevee + Island + DepthSD & 14 & 11386.3 & 11414.4 & 3.99 & 0.01 & 7.35 \\
\hline Null Model & 5 & 11601.0 & 11611.1 & 200.64 & $2.48 \mathrm{E}-45$ & $3.71 \mathrm{E}+43$ \\
\hline
\end{tabular}


Table 12. The ranking of candidate models at the pond scale for foraging and roosting piscivores in the former salt production ponds of South San Francisco Bay, California

[Models are ranked by differences in Akaike's information criterion and only models of $\triangle \mathrm{AIC}<4$ and the null model are presented. Birds were surveyed across the former salt ponds during October through April 2002-2015. Variables considered included the mean pond depth (Depth), the presence of islands (Island), variation in topography (DepthSD), \% of pond levee open to hunting (HuntLevee), \% of pond levee open to the public (OpenLevee), pond area (Area), water salinity, pond distance to urban areas (UrbanDist), and pond distance to the bay (BayDist). k: Number of estimated parameters in the model. AICc: Second-order Akaike's information criterion. $\triangle$ AICc: The difference between the AICc of the current model and the AICc of the top model. wi: Akaike weight-likelihood of the model relative to candidate models. Evidence ratio: The weight of evidence that the current model is inferior to the top model]

\begin{tabular}{|c|c|c|c|c|c|c|}
\hline Model name & k & $-2 \log L$ & $\mathrm{AICc}$ & $\triangle \mathrm{AICc}$ & $\mathbf{w}_{\mathrm{i}}$ & $\begin{array}{c}\text { Evidence } \\
\text { ratio }\end{array}$ \\
\hline \multicolumn{7}{|l|}{ Foraging } \\
\hline \multicolumn{7}{|l|}{ Depth + Salinity + BayDist + UrbanDist + Area + OpenLevee +} \\
\hline HuntLevee + Island + Breached & 14 & 12685.2 & 12713.4 & 0.00 & 0.24 & 1.00 \\
\hline \multicolumn{7}{|l|}{ Depth + Salinity + UrbanDist + Area + OpenLevee + HuntLevee + Island } \\
\hline+ Breached & 13 & 12688.3 & 12714.5 & 1.08 & 0.14 & 1.72 \\
\hline \multicolumn{7}{|l|}{ Depth + Salinity + BayDist + Area + OpenLevee + HuntLevee + Island +} \\
\hline Breached & 13 & 12689.2 & 12715.4 & 2.00 & 0.09 & 2.72 \\
\hline \multicolumn{7}{|l|}{ Depth + Salinity + BayDist + UrbanDist + Area + OpenLevee +} \\
\hline HuntLevee + Island + Breached + DepthSD & 15 & 12685.2 & 12715.4 & 2.00 & 0.09 & 2.72 \\
\hline Depth + Salinity + UrbanDist + Area + HuntLevee + Island + Breached & 12 & 12691.4 & 12715.6 & 2.18 & 0.08 & 2.98 \\
\hline \multicolumn{7}{|l|}{ Depth + Salinity + BayDist + UrbanDist + Area + HuntLevee + Island +} \\
\hline Breached & 13 & 12689.6 & 12715.7 & 2.32 & 0.08 & 3.19 \\
\hline \multicolumn{7}{|l|}{ Depth + Salinity + UrbanDist + Area + OpenLevee + HuntLevee + Island } \\
\hline+ Breached + DepthSD & 14 & 12688.1 & 12716.2 & 2.86 & 0.06 & 4.18 \\
\hline \multicolumn{7}{|l|}{ Depth + Salinity + BayDist + UrbanDist + Area + HuntLevee + Island +} \\
\hline Breached + DepthSD & 14 & 12688.9 & 12717.0 & 3.64 & 0.04 & 6.17 \\
\hline Null Model & 5 & 13062.0 & 13072.0 & 358.61 & $3.25 \mathrm{E}-79$ & $7.45 \mathrm{E}+77$ \\
\hline \multicolumn{7}{|l|}{ Roosting } \\
\hline \multicolumn{7}{|l|}{ Depth + Salinity + BayDist + UrbanDist + Area + OpenLevee + Island +} \\
\hline Breached & 13 & 15499.4 & 15525.6 & 0.00 & 0.11 & 1.00 \\
\hline \multicolumn{7}{|l|}{ Depth + Salinity + BayDist + UrbanDist + Area + OpenLevee +} \\
\hline HuntLevee + Island + Breached & 14 & 15497.9 & 15526.0 & 0.46 & 0.09 & 1.26 \\
\hline \multicolumn{7}{|l|}{ Depth + Salinity + BayDist + UrbanDist + Area + OpenLevee + Island +} \\
\hline Breached + DepthSD & 14 & 15498.3 & 15526.4 & 0.88 & 0.07 & 1.55 \\
\hline Depth + Salinity + UrbanDist + Area + OpenLevee + Island + Breached & 12 & 15502.3 & 15526.4 & 0.88 & 0.07 & 1.55 \\
\hline \multicolumn{7}{|l|}{ Depth + Salinity + UrbanDist + Area + OpenLevee + HuntLevee + Island } \\
\hline+ Breached & 13 & 15500.8 & 15526.9 & 1.34 & 0.06 & 1.95 \\
\hline \multicolumn{7}{|l|}{ Depth + Salinity + BayDist + UrbanDist + Area + Island + Breached +} \\
\hline DepthSD & 13 & 15500.8 & 15526.9 & 1.36 & 0.06 & 1.97 \\
\hline \multicolumn{7}{|l|}{ Depth + Salinity + BayDist + UrbanDist + Area + OpenLevee +} \\
\hline HuntLevee + Island + Breached + DepthSD & 15 & 15496.8 & 15526.9 & 1.38 & 0.06 & 1.99 \\
\hline \multicolumn{7}{|l|}{ Depth + Salinity + BayDist + UrbanDist + Area + HuntLevee + Island +} \\
\hline Breached + DepthSD & 14 & 15499.0 & 15527.2 & 1.64 & 0.05 & 2.27 \\
\hline Depth + Salinity + UrbanDist + Area + Island + Breached & 11 & 15505.3 & 15527.4 & 1.83 & 0.04 & 2.49 \\
\hline Depth + Salinity + UrbanDist + Area + HuntLevee + Island + Breached & 12 & 15503.4 & 15527.6 & 2.00 & 0.04 & 2.72 \\
\hline Depth + Salinity + BayDist + UrbanDist + Area + Island + Breached & 12 & 15503.5 & 15527.6 & 2.06 & 0.04 & 2.80 \\
\hline \multicolumn{7}{|l|}{ Depth + Salinity + BayDist + UrbanDist + Area + HuntLevee + Island +} \\
\hline Breached & 13 & 15501.6 & 15527.6 & 2.20 & 0.04 & 3.00 \\
\hline
\end{tabular}




\begin{tabular}{|c|c|c|c|c|c|c|}
\hline Model name & k & $-2 \log L$ & AlCc & $\triangle \mathrm{AICc}$ & $\mathbf{w}_{\mathrm{i}}$ & $\begin{array}{c}\text { Evidence } \\
\text { ratio }\end{array}$ \\
\hline \multicolumn{7}{|l|}{ Depth + Salinity + UrbanDist + Area + OpenLevee + Island + Breached } \\
\hline + DepthSD & 13 & 15502.1 & 15528.3 & 2.70 & 0.03 & 3.86 \\
\hline Depth + Salinity + UrbanDist + Area + Island + Breached + DepthSD & 12 & 15504.2 & 15528.4 & 2.80 & 0.03 & 4.06 \\
\hline \multicolumn{7}{|l|}{ Depth + Salinity + UrbanDist + Area + HuntLevee + Island + Breached +} \\
\hline DepthSD & 13 & 15502.5 & 15528.6 & 3.08 & 0.02 & 4.66 \\
\hline \multicolumn{7}{|l|}{ Depth + Salinity + UrbanDist + Area + OpenLevee + HuntLevee + Island } \\
\hline+ Breached + DepthSD & 14 & 15500.6 & 15528.7 & 3.18 & 0.02 & 4.90 \\
\hline Depth + Salinity + BayDist + Area + OpenLevee + Island + Breached & 12 & 15504.9 & 15529.0 & 3.48 & 0.02 & 5.70 \\
\hline \multicolumn{7}{|l|}{ Depth + Salinity + BayDist + Area + OpenLevee + HuntLevee + Island +} \\
\hline Breached & 13 & 15503.3 & 15529.5 & 3.92 & 0.02 & 7.10 \\
\hline Null Model & 5 & 15846.3 & 15856.4 & 330.81 & $1.61 \mathrm{E}-73$ & $6.84 \mathrm{E}+71$ \\
\hline
\end{tabular}


Table 13. The ranking of candidate models at the pond scale for foraging terns in the former salt production ponds of South San Francisco Bay, California.

[Models are ranked by differences in Akaike's information criterion and only models of $\triangle \mathrm{AIC}<4$ and the null model are presented. Birds were surveyed across the former salt ponds during October through April 2002-2015. Variables considered included the mean pond depth (Depth), the presence of islands (Island), variation in topography (DepthSD), \% of pond levee open to hunting (HuntLevee), \% of pond levee open to the public (OpenLevee), pond area (Area), water salinity, pond distance to urban areas (UrbanDist), and pond distance to the bay (BayDist). k: Number of estimated parameters in the model. AICc: Second-order Akaike's information criterion. $\triangle$ AICc: The difference between the AICc of the current model and the AICc of the top model. $\mathbf{w}_{\mathbf{i}}$ : Akaike weight-likelihood of the model relative to candidate models. Evidence ratio: The weight of evidence that the current model is inferior to the top model]

\begin{tabular}{|c|c|c|c|c|c|c|}
\hline Model name & k & $-2 \log L$ & AICc & $\triangle \mathrm{AICc}$ & $w_{i}$ & $\begin{array}{c}\text { Evidence } \\
\text { ratio }\end{array}$ \\
\hline Depth + Salinity + BayDist + Area + Island + Breached & 11 & 5488.4 & 5510.5 & 0.00 & 0.03 & 1.00 \\
\hline Depth + Salinity + BayDist + Area + HuntLevee + Island + Breached & 12 & 5486.5 & 5510.6 & 0.16 & 0.02 & 1.08 \\
\hline \multicolumn{7}{|l|}{ Depth + Salinity + BayDist + UrbanDist + Area + HuntLevee + Island +} \\
\hline Breached & 13 & 5484.6 & 5510.7 & 0.21 & 0.02 & 1.11 \\
\hline Depth + Salinity + BayDist + UrbanDist + Area + Island + Breached & 12 & 5486.6 & 5510.7 & 0.22 & 0.02 & 1.11 \\
\hline Salinity + BayDist + UrbanDist + Area + HuntLevee + Island + Breached & 12 & 5486.6 & 5510.7 & 0.22 & 0.02 & 1.11 \\
\hline Salinity + BayDist + UrbanDist + Area + Island + Breached & 11 & 5488.7 & 5510.8 & 0.32 & 0.02 & 1.17 \\
\hline Salinity + BayDist + Area + Island + Breached & 10 & 5491.0 & 5511.1 & 0.60 & 0.02 & 1.35 \\
\hline Salinity + BayDist + Area + HuntLevee + Island + Breached & 11 & 5489.0 & 5511.1 & 0.66 & 0.02 & 1.39 \\
\hline Salinity + BayDist + Area + OpenLevee + HuntLevee + Island & 11 & 5489.1 & 5511.2 & 0.70 & 0.02 & 1.42 \\
\hline Salinity + UrbanDist + Area + HuntLevee + Island + Breached & 11 & 5489.1 & 5511.2 & 0.72 & 0.02 & 1.43 \\
\hline Salinity + UrbanDist + Area + Island + Breached & 10 & 5491.4 & 5511.5 & 0.98 & 0.02 & 1.64 \\
\hline Salinity + BayDist + UrbanDist + Area + OpenLevee + HuntLevee + Island & 12 & 5487.4 & 5511.5 & 1.02 & 0.02 & 1.66 \\
\hline \multicolumn{7}{|l|}{ Salinity + BayDist + UrbanDist + Area + OpenLevee + HuntLevee + Island } \\
\hline+ Breached & 13 & 5485.4 & 5511.5 & 1.05 & 0.02 & 1.69 \\
\hline Salinity + BayDist + Area + OpenLevee + HuntLevee + Island + Breached & 12 & 5487.5 & 5511.6 & 1.10 & 0.02 & 1.73 \\
\hline Salinity + BayDist + UrbanDist + Area + HuntLevee + Island & 11 & 5489.5 & 5511.6 & 1.16 & 0.01 & 1.79 \\
\hline Salinity + BayDist + UrbanDist + Area + Island & 10 & 5491.6 & 5511.7 & 1.18 & 0.01 & 1.81 \\
\hline Salinity + BayDist + Area + Island & 9 & 5493.6 & 5511.7 & 1.19 & 0.01 & 1.81 \\
\hline Salinity + BayDist + Area + HuntLevee + Island & 10 & 5491.7 & 5511.8 & 1.30 & 0.01 & 1.92 \\
\hline \multicolumn{7}{|l|}{ Depth + Salinity + BayDist + Area + OpenLevee + HuntLevee + Island +} \\
\hline Breached & 13 & 5485.7 & 5511.8 & 1.33 & 0.01 & 1.95 \\
\hline Depth + Salinity + BayDist + Area + Island & 10 & 5491.7 & 5511.8 & 1.34 & 0.01 & 1.96 \\
\hline Salinity + BayDist + Area + OpenLevee + Island & 10 & 5491.8 & 5511.9 & 1.38 & 0.01 & 2.00 \\
\hline Depth + Salinity + BayDist + Area + HuntLevee + Island & 11 & 5489.9 & 5512.0 & 1.52 & 0.01 & 2.14 \\
\hline \multicolumn{7}{|l|}{ Depth + Salinity + BayDist + UrbanDist + Area + OpenLevee + HuntLevee } \\
\hline+ Island + Breached & 14 & 5483.9 & 5512.1 & 1.61 & 0.01 & 2.24 \\
\hline Depth + Salinity + BayDist + Area + OpenLevee + Island + Breached & 12 & 5488.0 & 5512.1 & 1.62 & 0.01 & 2.24 \\
\hline Depth + Salinity + BayDist + Area + OpenLevee + HuntLevee + Island & 12 & 5488.0 & 5512.1 & 1.66 & 0.01 & 2.29 \\
\hline Salinity + BayDist + Area + OpenLevee + Island + Breached & 11 & 5490.0 & 5512.1 & 1.66 & 0.01 & 2.29 \\
\hline Salinity + BayDist + UrbanDist + Area + OpenLevee + Island + Breached & 12 & 5488.1 & 5512.2 & 1.70 & 0.01 & 2.34 \\
\hline Depth + Salinity + BayDist + UrbanDist + Area + Island & 11 & 5490.1 & 5512.2 & 1.76 & 0.01 & 2.41 \\
\hline Salinity + BayDist + UrbanDist + Area + OpenLevee + Island & 11 & 5490.1 & 5512.2 & 1.76 & 0.01 & 2.41 \\
\hline Depth + Salinity + BayDist + UrbanDist + Area + HuntLevee + Island & 12 & 5488.2 & 5512.3 & 1.80 & 0.01 & 2.46 \\
\hline Depth + Salinity + BayDist + Area + Island + Breached + DepthSD & 12 & 5488.2 & 5512.3 & 1.82 & 0.01 & 2.48 \\
\hline \multicolumn{7}{|l|}{ Depth + Salinity + BayDist + UrbanDist + Area + OpenLevee + Island +} \\
\hline Breached & 13 & 5486.3 & 5512.5 & 1.99 & 0.01 & 2.71 \\
\hline
\end{tabular}




\begin{tabular}{|c|c|c|c|c|c|c|}
\hline Model name & k & $-2 \log L$ & AICc & $\triangle \mathrm{AICC}$ & $\mathbf{w}_{\mathrm{i}}$ & $\begin{array}{c}\text { Evidence } \\
\text { ratio }\end{array}$ \\
\hline \multicolumn{7}{|l|}{ Depth + Salinity + BayDist + Area + HuntLevee + Island + Breached +} \\
\hline DepthSD & 13 & 5486.4 & 5512.5 & 2.03 & 0.01 & 2.77 \\
\hline \multicolumn{7}{|l|}{ Depth + Salinity + BayDist + UrbanDist + Area + Island + Breached +} \\
\hline DepthSD & 13 & 5486.4 & 5512.6 & 2.07 & 0.01 & 2.82 \\
\hline \multicolumn{7}{|l|}{ Depth + Salinity + BayDist + UrbanDist + Area + HuntLevee + Island +} \\
\hline Breached + DepthSD & 14 & 5484.5 & 5512.6 & 2.13 & 0.01 & 2.91 \\
\hline Depth + Salinity + UrbanDist + Area + HuntLevee + Island + Breached & 12 & 5488.5 & 5512.6 & 2.14 & 0.01 & 2.91 \\
\hline Depth + Salinity + BayDist + Area + OpenLevee + Island & 11 & 5490.5 & 5512.6 & 2.14 & 0.01 & 2.92 \\
\hline Salinity + Area + HuntLevee + Island + Breached & 10 & 5492.6 & 5512.6 & 2.16 & 0.01 & 2.95 \\
\hline \multicolumn{7}{|l|}{ Depth + Salinity + BayDist + UrbanDist + Area + OpenLevee + HuntLevee } \\
\hline+ Island & 13 & 5486.6 & 5512.7 & 2.23 & 0.01 & 3.06 \\
\hline \multicolumn{7}{|l|}{ Salinity + BayDist + UrbanDist + Area + HuntLevee + Island + Breached +} \\
\hline DepthSD & 13 & 5486.6 & 5512.7 & 2.23 & 0.01 & 3.06 \\
\hline Salinity + UrbanDist + Area + HuntLevee + Island + Breached + DepthSD & 12 & 5488.6 & 5512.7 & 2.24 & 0.01 & 3.06 \\
\hline Salinity + Area + Island + Breached & 9 & 5494.7 & 5512.8 & 2.29 & 0.01 & 3.14 \\
\hline Salinity + UrbanDist + Area + HuntLevee + Island & 10 & 5492.7 & 5512.8 & 2.30 & 0.01 & 3.17 \\
\hline Salinity + BayDist + UrbanDist + Area + Island + Breached + DepthSD & 12 & 5488.7 & 5512.8 & 2.32 & 0.01 & 3.18 \\
\hline Salinity + BayDist + Area + OpenLevee + HuntLevee + Island + DepthSD & 12 & 5488.7 & 5512.8 & 2.32 & 0.01 & 3.18 \\
\hline \multicolumn{7}{|l|}{ Salinity + UrbanDist + Area + OpenLevee + HuntLevee + Island +} \\
\hline Breached & 12 & 5488.7 & 5512.9 & 2.38 & 0.01 & 3.28 \\
\hline Depth + Salinity + UrbanDist + Area + Island + Breached & 11 & 5490.8 & 5512.9 & 2.38 & 0.01 & 3.29 \\
\hline Salinity + UrbanDist + Area + Island + Breached + DepthSD & 11 & 5490.8 & 5512.9 & 2.38 & 0.01 & 3.29 \\
\hline Salinity + UrbanDist + Area + Island & 9 & 5495.0 & 5513.0 & 2.55 & 0.01 & 3.58 \\
\hline Salinity + BayDist + Area + Island + Breached + DepthSD & 11 & 5491.0 & 5513.1 & 2.62 & 0.01 & 3.71 \\
\hline Salinity + BayDist + Area + HuntLevee + Island + Breached + DepthSD & 12 & 5489.0 & 5513.2 & 2.68 & 0.01 & 3.81 \\
\hline \multicolumn{7}{|l|}{ Salinity + BayDist + UrbanDist + Area + OpenLevee + HuntLevee + Island } \\
\hline + DepthSD & 13 & 5487.1 & 5513.2 & 2.71 & 0.01 & 3.89 \\
\hline Depth + Salinity + BayDist + UrbanDist + Area + OpenLevee + Island & 12 & 5489.2 & 5513.3 & 2.80 & 0.01 & 4.05 \\
\hline \multicolumn{7}{|l|}{ Depth + Salinity + BayDist + Area + OpenLevee + HuntLevee + Island +} \\
\hline Breached + DepthSD & 14 & 5485.1 & 5513.3 & 2.81 & 0.01 & 4.08 \\
\hline \multicolumn{7}{|l|}{ Salinity + BayDist + Area + OpenLevee + HuntLevee + Island + Breached +} \\
\hline DepthSD & 13 & 5487.2 & 5513.3 & 2.81 & 0.01 & 4.09 \\
\hline \multicolumn{7}{|l|}{ Salinity + BayDist + UrbanDist + Area + OpenLevee + HuntLevee + Island } \\
\hline+ Breached + DepthSD & 14 & 5485.2 & 5513.4 & 2.87 & 0.01 & 4.21 \\
\hline Salinity + UrbanDist + Area + OpenLevee + Island + Breached & 11 & 5491.3 & 5513.4 & 2.92 & 0.01 & 4.31 \\
\hline Salinity + BayDist + Area + OpenLevee + Island + DepthSD & 11 & 5491.4 & 5513.5 & 3.02 & 0.01 & 4.53 \\
\hline \multicolumn{7}{|l|}{ Depth + Salinity + BayDist + Area + OpenLevee + HuntLevee + Island +} \\
\hline DepthSD & 13 & 5487.4 & 5513.5 & 3.03 & 0.01 & 4.56 \\
\hline \multicolumn{7}{|l|}{ Depth + Salinity + BayDist + Area + OpenLevee + Island + Breached +} \\
\hline DepthSD & 13 & 5487.5 & 5513.6 & 3.11 & 0.01 & 4.75 \\
\hline \multicolumn{7}{|l|}{ Depth + Salinity + UrbanDist + Area + HuntLevee + Island + Breached +} \\
\hline DepthSD & 13 & 5487.5 & 5513.6 & 3.11 & 0.01 & 4.75 \\
\hline Depth + Salinity + UrbanDist + Area + Island + Breached + DepthSD & 12 & 5489.5 & 5513.6 & 3.12 & 0.01 & 4.75 \\
\hline Salinity + BayDist + UrbanDist + Area + HuntLevee + Island + DepthSD & 12 & 5489.5 & 5513.7 & 3.18 & 0.01 & 4.90 \\
\hline Salinity + BayDist + UrbanDist + Area + Island + DepthSD & 11 & 5491.6 & 5513.7 & 3.20 & 0.01 & 4.95 \\
\hline Salinity + BayDist + Area + Island + DepthSD & 10 & 5493.6 & 5513.7 & 3.20 & 0.01 & 4.96 \\
\hline
\end{tabular}




\begin{tabular}{|c|c|c|c|c|c|c|}
\hline Model name & k & $-2 \log L$ & $\mathrm{AICc}$ & $\triangle \mathrm{AICc}$ & $w_{i}$ & $\begin{array}{c}\text { Evidence } \\
\text { ratio }\end{array}$ \\
\hline \multicolumn{7}{|l|}{ Salinity + UrbanDist + Area + OpenLevee + HuntLevee + Island +} \\
\hline Breached + DepthSD & 13 & 5487.6 & 5513.7 & 3.23 & 0.01 & 5.04 \\
\hline \multicolumn{7}{|l|}{ Depth + Salinity + BayDist + UrbanDist + Area + OpenLevee + HuntLevee } \\
\hline+ Island + Breached + DepthSD & 15 & 5483.6 & 5513.7 & 3.26 & 0.01 & 5.09 \\
\hline Depth + Salinity + BayDist + Area + Island + DepthSD & 11 & 5491.6 & 5513.7 & 3.26 & 0.01 & 5.10 \\
\hline Salinity + BayDist + Area + HuntLevee + Island + DepthSD & 11 & 5491.7 & 5513.8 & 3.32 & 0.01 & 5.26 \\
\hline Salinity + UrbanDist + Area + OpenLevee + HuntLevee + Island & 11 & 5491.8 & 5513.9 & 3.40 & 0.00 & 5.47 \\
\hline Depth + Salinity + Area + HuntLevee + Island + Breached & 11 & 5491.8 & 5513.9 & 3.42 & 0.00 & 5.53 \\
\hline Salinity + BayDist + Area + OpenLevee + Island + Breached + DepthSD & 12 & 5489.8 & 5513.9 & 3.44 & 0.00 & 5.58 \\
\hline Salinity + Area + Island + Breached + DepthSD & 10 & 5493.8 & 5513.9 & 3.44 & 0.00 & 5.60 \\
\hline Salinity + Area + HuntLevee + Island & 9 & 5495.9 & 5513.9 & 3.47 & 0.00 & 5.67 \\
\hline Depth + Salinity + BayDist + Area + HuntLevee + Island + DepthSD & 12 & 5489.8 & 5514.0 & 3.48 & 0.00 & 5.69 \\
\hline Salinity + BayDist + UrbanDist + Area + OpenLevee + Island + DepthSD & 12 & 5489.8 & 5514.0 & 3.48 & 0.00 & 5.69 \\
\hline Depth + Salinity + Area + Island + Breached & 10 & 5493.9 & 5514.0 & 3.48 & 0.00 & 5.71 \\
\hline Salinity + Area + HuntLevee + Island + Breached + DepthSD & 11 & 5491.9 & 5514.0 & 3.50 & 0.00 & 5.75 \\
\hline Depth + Salinity + BayDist + Area + OpenLevee + Island + DepthSD & 12 & 5489.9 & 5514.0 & 3.52 & 0.00 & 5.80 \\
\hline \multicolumn{7}{|l|}{ Salinity + BayDist + UrbanDist + Area + OpenLevee + Island + Breached +} \\
\hline DepthSD & 13 & 5487.9 & 5514.0 & 3.53 & 0.00 & 5.86 \\
\hline Salinity + Area + Island & 8 & 5498.0 & 5514.0 & 3.56 & 0.00 & 5.92 \\
\hline \multicolumn{7}{|l|}{ Depth + Salinity + BayDist + UrbanDist + Area + OpenLevee + Island +} \\
\hline Breached + DepthSD & 14 & 5486.0 & 5514.1 & 3.63 & 0.00 & 6.15 \\
\hline Salinity + Area + OpenLevee + HuntLevee + Island + Breached & 11 & 5492.1 & 5514.2 & 3.68 & 0.00 & 6.30 \\
\hline Depth + Salinity + BayDist + UrbanDist + Area + Island + DepthSD & 12 & 5490.1 & 5514.2 & 3.70 & 0.00 & 6.35 \\
\hline \multicolumn{7}{|l|}{ Salinity + UrbanDist + Area + OpenLevee + HuntLevee + Island +} \\
\hline DepthSD & 12 & 5490.1 & 5514.2 & 3.70 & 0.00 & 6.35 \\
\hline \multicolumn{7}{|l|}{ Depth + Salinity + BayDist + UrbanDist + Area + OpenLevee + HuntLevee } \\
\hline + Island + DepthSD & 14 & 5486.1 & 5514.2 & 3.75 & 0.00 & 6.54 \\
\hline Salinity + UrbanDist + Area + HuntLevee + Island + DepthSD & 11 & 5492.1 & 5514.2 & 3.76 & 0.00 & 6.55 \\
\hline \multicolumn{7}{|l|}{ Depth + Salinity + BayDist + UrbanDist + Area + HuntLevee + Island +} \\
\hline DepthSD & 13 & 5488.1 & 5514.3 & 3.77 & 0.00 & 6.60 \\
\hline Salinity + UrbanDist + Area + OpenLevee + Island + Breached + DepthSD & 12 & 5490.2 & 5514.3 & 3.84 & 0.00 & 6.81 \\
\hline Depth + Salinity + Area + Island + Breached + DepthSD & 11 & 5492.2 & 5514.3 & 3.84 & 0.00 & 6.82 \\
\hline Salinity + UrbanDist + Area + Island + DepthSD & 10 & 5494.2 & 5514.3 & 3.84 & 0.00 & 6.84 \\
\hline Null Model & 5 & 5585.2 & 5595.3 & 84.79 & $1.03 \mathrm{E}-20$ & $2.58 \mathrm{E}+18$ \\
\hline
\end{tabular}


Table 14. The ranking of candidate models at the pond scale for roosting terns in the former salt production ponds of South San Francisco Bay, California.

[Models are ranked by differences in Akaike's information criterion and only models of $\triangle \mathrm{AIC}<4$ and the null model are presented. Birds were surveyed across the former salt ponds during October through April 2002-2015. Variables considered included the mean pond depth (Depth), the presence of islands (Island), variation in topography (DepthSD), \% of pond levee open to hunting (HuntLevee), \% of pond levee open to the public (OpenLevee), pond area (Area), water salinity, pond distance to urban areas (UrbanDist), and pond distance to the bay (BayDist). k: Number of estimated parameters in the model. AICc: Second-order Akaike's information criterion. $\triangle$ AICc: The difference between the AICc of the current model and the AICc of the top model. wi: Akaike weight-likelihood of the model relative to candidate models. Evidence ratio: The weight of evidence that the current model is inferior to the top model]

\begin{tabular}{|c|c|c|c|c|c|c|}
\hline Model name & k & $-2 \log L$ & AICc & $\triangle \mathrm{AICc}$ & $\mathbf{w}_{\mathrm{i}}$ & $\begin{array}{c}\text { Evidence } \\
\text { ratio }\end{array}$ \\
\hline Salinity + BayDist + Area + OpenLevee + HuntLevee + Island & 11 & 7217.5 & 7239.6 & 0.00 & 0.07 & 1.00 \\
\hline Salinity + Area + OpenLevee + HuntLevee + Island & 10 & 7220.0 & 7240.1 & 0.48 & 0.06 & 1.27 \\
\hline Depth + Salinity + Area + OpenLevee + HuntLevee + Island & 11 & 7218.3 & 7240.3 & 0.70 & 0.05 & 1.42 \\
\hline Salinity + Area + OpenLevee + HuntLevee + Island + Breached & 11 & 7218.3 & 7240.3 & 0.70 & 0.05 & 1.42 \\
\hline Salinity + BayDist + Area + OpenLevee + HuntLevee + Island + Breached & 12 & 7216.3 & 7240.5 & 0.82 & 0.05 & 1.50 \\
\hline Depth + Salinity + BayDist + Area + OpenLevee + HuntLevee + Island & 12 & 7216.6 & 7240.7 & 1.08 & 0.04 & 1.71 \\
\hline Salinity + BayDist + UrbanDist + Area + OpenLevee + HuntLevee + Island & 12 & 7217.0 & 7241.1 & 1.50 & 0.03 & 2.11 \\
\hline Salinity + Area + HuntLevee + Island + Breached & 10 & 7221.1 & 7241.2 & 1.56 & 0.03 & 2.19 \\
\hline Depth + Salinity + Area + OpenLevee + HuntLevee + Island + Breached & 12 & 7217.3 & 7241.4 & 1.78 & 0.03 & 2.43 \\
\hline Salinity + BayDist + Area + OpenLevee + HuntLevee + Island + DepthSD & 12 & 7217.5 & 7241.6 & 1.94 & 0.03 & 2.63 \\
\hline Salinity + UrbanDist + Area + OpenLevee + HuntLevee + Island & 11 & 7219.9 & 7242.0 & 2.36 & 0.02 & 3.25 \\
\hline Salinity + Area + OpenLevee + HuntLevee + Island + DepthSD & 11 & 7219.9 & 7242.0 & 2.38 & 0.02 & 3.29 \\
\hline \multicolumn{7}{|l|}{ Depth + Salinity + BayDist + Area + OpenLevee + HuntLevee + Island +} \\
\hline Breached & 13 & 7215.9 & 7242.0 & 2.39 & 0.02 & 3.31 \\
\hline \multicolumn{7}{|l|}{ Salinity + BayDist + UrbanDist + Area + OpenLevee + HuntLevee + Island } \\
\hline+ Breached & 13 & 7215.9 & 7242.0 & 2.39 & 0.02 & 3.31 \\
\hline Salinity + UrbanDist + Area + OpenLevee + HuntLevee + Island + Breached & 12 & 7218.1 & 7242.2 & 2.60 & 0.02 & 3.66 \\
\hline Depth + Salinity + UrbanDist + Area + OpenLevee + HuntLevee + Island & 12 & 7218.2 & 7242.3 & 2.66 & 0.02 & 3.77 \\
\hline \multicolumn{7}{|l|}{ Salinity + BayDist + Area + OpenLevee + HuntLevee + Island + Breached +} \\
\hline DepthSD & 13 & 7216.2 & 7242.3 & 2.67 & 0.02 & 3.81 \\
\hline Salinity + Area + OpenLevee + HuntLevee + Island + Breached + DepthSD & 12 & 7218.2 & 7242.3 & 2.70 & 0.02 & 3.85 \\
\hline Depth + Salinity + Area + OpenLevee + HuntLevee + Island + DepthSD & 12 & 7218.2 & 7242.4 & 2.72 & 0.02 & 3.89 \\
\hline \multicolumn{7}{|l|}{ Depth + Salinity + BayDist + UrbanDist + Area + OpenLevee + HuntLevee } \\
\hline+ Island & 13 & 7216.3 & 7242.4 & 2.77 & 0.02 & 4.00 \\
\hline Salinity + BayDist + Area + HuntLevee + Island + Breached & 11 & 7220.4 & 7242.5 & 2.82 & 0.02 & 4.10 \\
\hline \multicolumn{7}{|l|}{ Depth + Salinity + BayDist + Area + OpenLevee + HuntLevee + Island +} \\
\hline DepthSD & 13 & 7216.3 & 7242.5 & 2.83 & 0.02 & 4.13 \\
\hline Salinity + Area + HuntLevee + Island & 9 & 7224.4 & 7242.5 & 2.85 & 0.02 & 4.16 \\
\hline Salinity + Area + HuntLevee + Island + Breached + DepthSD & 11 & 7220.7 & 7242.8 & 3.14 & 0.01 & 4.81 \\
\hline Depth + Salinity + Area + HuntLevee + Island + Breached & 11 & 7220.7 & 7242.8 & 3.18 & 0.01 & 4.90 \\
\hline \multicolumn{7}{|l|}{ Salinity + BayDist + UrbanDist + Area + OpenLevee + HuntLevee + Island } \\
\hline + DepthSD & 13 & 7217.0 & 7243.1 & 3.45 & 0.01 & 5.63 \\
\hline Salinity + BayDist + Area + HuntLevee + Island + Breached + DepthSD & 12 & 7219.0 & 7243.1 & 3.48 & 0.01 & 5.69 \\
\hline Salinity + UrbanDist + Area + HuntLevee + Island + Breached & 11 & 7221.1 & 7243.2 & 3.54 & 0.01 & 5.87 \\
\hline \multicolumn{7}{|l|}{ Depth + Salinity + UrbanDist + Area + OpenLevee + HuntLevee + Island +} \\
\hline Breached & 13 & 7217.2 & 7243.4 & 3.73 & 0.01 & 6.47 \\
\hline \multicolumn{7}{|l|}{ Depth + Salinity + Area + OpenLevee + HuntLevee + Island + Breached +} \\
\hline DepthSD & 13 & 7217.3 & 7243.4 & 3.77 & 0.01 & 6.60 \\
\hline
\end{tabular}




\begin{tabular}{lrrrrrr}
\hline \multicolumn{1}{c}{ Model name } & k & -2LogL & AICc & $\Delta$ AICc & W & $\begin{array}{c}\text { Evidence } \\
\text { ratio }\end{array}$ \\
\hline Salinity + BayDist + Area + HuntLevee + Island & 10 & 7223.4 & 7243.4 & 3.80 & 0.01 & 6.70 \\
Depth + Salinity + Area + HuntLevee + Island & 10 & 7223.4 & 7243.5 & 3.82 & 0.01 & 6.77 \\
Null Model & 5 & 7302.1 & 7312.1 & 72.45 & $1.32 \mathrm{E}-17$ & $5.4 \mathrm{E}+15$ \\
\hline
\end{tabular}


Table 15. The ranking of candidate models at the pond scale for foraging gulls in the former salt production ponds of South San Francisco Bay, California.

[Models are ranked by differences in Akaike's information criterion and only models of $\triangle \mathrm{AIC}<4$ and the null model are presented. Birds were surveyed across the former salt ponds during October through April 2002-2015. Variables considered included the mean pond depth (Depth), the presence of islands (Island), variation in topography (DepthSD), \% of pond levee open to hunting (HuntLevee), \% of pond levee open to the public (OpenLevee), pond area (Area), water salinity, pond distance to urban areas (UrbanDist), and pond distance to the bay (BayDist). k: Number of estimated parameters in the model. AICc: Second-order Akaike's information criterion. $\triangle$ AICc: The difference between the AICc of the current model and the AICc of the top model. wi: Akaike weight-likelihood of the model relative to candidate models. Evidence ratio: The weight of evidence that the current model is inferior to the top model]

\begin{tabular}{|c|c|c|c|c|c|c|}
\hline Model name & k & $-2 \log L$ & AICc & $\triangle \mathrm{AICc}$ & $\mathbf{w i}_{\mathbf{i}}$ & $\begin{array}{c}\text { Evidence } \\
\text { ratio }\end{array}$ \\
\hline \multicolumn{7}{|l|}{ Depth + Salinity + BayDist + UrbanDist + Area + OpenLevee + HuntLevee + Island +} \\
\hline DistLandfill + Breached + DepthSD & 16 & 11006.0 & 11038.2 & 0.00 & 0.09 & 1.00 \\
\hline \multicolumn{7}{|l|}{ Depth + Salinity + BayDist + Area + OpenLevee + HuntLevee + Island + DistLandfill } \\
\hline+ Breached & 14 & 11010.4 & 11038.6 & 0.38 & 0.07 & 1.21 \\
\hline \multicolumn{7}{|l|}{ Depth + Salinity + BayDist + UrbanDist + Area + OpenLevee + HuntLevee + Island +} \\
\hline DistLandfill + Breached & 15 & 11008.5 & 11038.6 & 0.44 & 0.07 & 1.24 \\
\hline Depth + Salinity + BayDist + Area + HuntLevee + Island + DistLandfill + Breached & 13 & 11012.6 & 11038.8 & 0.56 & 0.07 & 1.32 \\
\hline \multicolumn{7}{|l|}{ Depth + Salinity + BayDist + UrbanDist + Area + HuntLevee + Island + DistLandfill } \\
\hline+ Breached & 14 & 11010.8 & 11038.9 & 0.72 & 0.06 & 1.43 \\
\hline \multicolumn{7}{|l|}{ Depth + Salinity + BayDist + UrbanDist + Area + OpenLevee + HuntLevee + Island +} \\
\hline DistLandfill + DepthSD & 15 & 11008.8 & 11039.0 & 0.76 & 0.06 & 1.46 \\
\hline \multicolumn{7}{|l|}{ Depth + Salinity + BayDist + Area + OpenLevee + HuntLevee + Island + DistLandfill } \\
\hline+ Breached + DepthSD & 15 & 11008.8 & 11039.0 & 0.80 & 0.06 & 1.49 \\
\hline \multicolumn{7}{|l|}{ Depth + Salinity + BayDist + Area + OpenLevee + HuntLevee + Island + DistLandfill } \\
\hline+ DepthSD & 14 & 11011.9 & 11040.0 & 1.82 & 0.04 & 2.48 \\
\hline \multicolumn{7}{|l|}{ Depth + Salinity + BayDist + UrbanDist + Area + OpenLevee + HuntLevee + Island +} \\
\hline DistLandfill & 14 & 11011.9 & 11040.1 & 1.88 & 0.03 & 2.56 \\
\hline Depth + Salinity + BayDist + Area + OpenLevee + HuntLevee + Island + DistLandfill & 13 & 11014.0 & 11040.1 & 1.92 & 0.03 & 2.61 \\
\hline \multicolumn{7}{|l|}{ Depth + Salinity + BayDist + UrbanDist + Area + HuntLevee + Island + DistLandfill } \\
\hline+ Breached + DepthSD & 15 & 11010.0 & 11040.2 & 1.98 & 0.03 & 2.69 \\
\hline \multicolumn{7}{|l|}{ Depth + Salinity + BayDist + Area + HuntLevee + Island + DistLandfill + Breached +} \\
\hline DepthSD & 14 & 11012.3 & 11040.4 & 2.20 & 0.03 & 3.00 \\
\hline Depth + Salinity + BayDist + Area + OpenLevee + HuntLevee + Island + Breached & 13 & 11014.3 & 11040.4 & 2.20 & 0.03 & 3.00 \\
\hline Depth + Salinity + BayDist + Area + OpenLevee + HuntLevee + Island & 12 & 11017.5 & 11041.6 & 3.38 & 0.02 & 5.42 \\
\hline Depth + Salinity + BayDist + Area + HuntLevee + Island + DistLandfill & 12 & 11017.6 & 11041.7 & 3.54 & 0.02 & 5.87 \\
\hline \multicolumn{7}{|l|}{ Depth + Salinity + BayDist + Area + OpenLevee + HuntLevee + Island + Breached +} \\
\hline DepthSD & 14 & 11013.6 & 11041.8 & 3.56 & 0.02 & 5.92 \\
\hline \multicolumn{7}{|l|}{ Depth + Salinity + BayDist + UrbanDist + Area + OpenLevee + HuntLevee + Island +} \\
\hline Breached & 14 & 11013.6 & 11041.8 & 3.58 & 0.01 & 5.98 \\
\hline Depth + Salinity + BayDist + UrbanDist + Area + HuntLevee + Island + DistLandfill & 13 & 11015.7 & 11041.8 & 3.60 & 0.01 & 6.04 \\
\hline
\end{tabular}


Table 16. The ranking of candidate models at the pond scale for roosting gulls in the former salt production ponds of South San Francisco Bay, California.

[Models are ranked by differences in Akaike's information criterion and only models of $\triangle \mathrm{AIC}<4$ and the null model are presented. Birds were surveyed across the former salt ponds during October through April 2002-2015. Variables considered included the mean pond depth (Depth), the presence of islands (Island), variation in topography (DepthSD), \% of pond levee open to hunting (HuntLevee), \% of pond levee open to the public (OpenLevee), pond area (Area), water salinity, pond distance to urban areas (UrbanDist), and pond distance to the bay (BayDist). k: Number of estimated parameters in the model. AICc: Second-order Akaike's information criterion. $\triangle$ AICc: The difference between the AICc of the current model and the AICc of the top model. wi: Akaike weight-likelihood of the model relative to candidate models. Evidence ratio: The weight of evidence that the current model is inferior to the top model]

\begin{tabular}{|c|c|c|c|c|c|c|}
\hline Model name & k & $-2 \log L$ & $\mathrm{AICc}$ & $\triangle \mathrm{AICc}$ & Wi & $\begin{array}{c}\text { Evidence } \\
\text { ratio }\end{array}$ \\
\hline \multicolumn{7}{|l|}{ Depth + Salinity + BayDist + Area + OpenLevee + HuntLevee + Island + } \\
\hline DistLandfill + Breached + DepthSD & 15 & 24567.8 & 24598.0 & 0.00 & 0.10 & 1.00 \\
\hline \multicolumn{7}{|l|}{ Depth + Salinity + BayDist + Area + OpenLevee + Island + DistLandfill +} \\
\hline Breached + DepthSD & 14 & 24570.0 & 24598.1 & 0.18 & 0.09 & 1.09 \\
\hline \multicolumn{7}{|l|}{ Depth + BayDist + Area + OpenLevee + Island + DistLandfill + Breached +} \\
\hline DepthSD & 13 & 24572.8 & 24598.9 & 0.96 & 0.06 & 1.62 \\
\hline \multicolumn{7}{|l|}{ Depth + BayDist + Area + OpenLevee + HuntLevee + Island + DistLandfill } \\
\hline + Breached + DepthSD & 14 & 24570.8 & 24598.9 & 0.98 & 0.06 & 1.63 \\
\hline \multicolumn{7}{|l|}{ Depth + Salinity + BayDist + UrbanDist + Area + OpenLevee + HuntLevee } \\
\hline + Island + DistLandfill + Breached + DepthSD & 16 & 24567.4 & 24599.6 & 1.62 & 0.04 & 2.25 \\
\hline \multicolumn{7}{|l|}{ Depth + Salinity + BayDist + Area + OpenLevee + HuntLevee + Island +} \\
\hline DistLandfill + Breached & 14 & 24571.6 & 24599.7 & 1.78 & 0.04 & 2.43 \\
\hline \multicolumn{7}{|l|}{ Depth + Salinity + BayDist + UrbanDist + Area + OpenLevee + Island +} \\
\hline DistLandfill + Breached + DepthSD & 15 & 24569.6 & 24599.8 & 1.80 & 0.04 & 2.46 \\
\hline Depth + BayDist + Area + OpenLevee + Island + DistLandfill + DepthSD & 12 & 24575.8 & 24599.9 & 1.94 & 0.04 & 2.64 \\
\hline \multicolumn{7}{|l|}{ Depth + BayDist + Area + OpenLevee + HuntLevee + Island + DistLandfill } \\
\hline + DepthSD & 13 & 24573.8 & 24599.9 & 1.96 & 0.04 & 2.66 \\
\hline \multicolumn{7}{|l|}{ Depth + Salinity + BayDist + Area + OpenLevee + Island + DistLandfill +} \\
\hline Breached & 13 & 24574.0 & 24600.1 & 2.16 & 0.03 & 2.94 \\
\hline \multicolumn{7}{|l|}{ Depth + Salinity + BayDist + Area + OpenLevee + HuntLevee + Island +} \\
\hline DistLandfill + DepthSD & 14 & 24572.0 & 24600.1 & 2.18 & 0.03 & 2.97 \\
\hline \multicolumn{7}{|l|}{ Depth + Salinity + BayDist + Area + OpenLevee + Island + DistLandfill +} \\
\hline DepthSD & 13 & 24574.2 & 24600.3 & 2.36 & 0.03 & 3.25 \\
\hline \multicolumn{7}{|l|}{ Depth + BayDist + UrbanDist + Area + OpenLevee + Island + DistLandfill +} \\
\hline Breached + DepthSD & 14 & 24572.4 & 24600.5 & 2.58 & 0.03 & 3.63 \\
\hline \multicolumn{7}{|l|}{ Depth + BayDist + UrbanDist + Area + OpenLevee + HuntLevee + Island +} \\
\hline DistLandfill + Breached + DepthSD & 15 & 24570.4 & 24600.6 & 2.60 & 0.03 & 3.67 \\
\hline \multicolumn{7}{|l|}{ Depth + BayDist + Area + OpenLevee + HuntLevee + Island + DistLandfill } \\
\hline+ Breached & 13 & 24575.0 & 24601.1 & 3.16 & 0.02 & 4.85 \\
\hline Depth + BayDist + Area + OpenLevee + Island + DistLandfill + Breached & 12 & 24577.2 & 24601.3 & 3.34 & 0.02 & 5.32 \\
\hline \multicolumn{7}{|l|}{ Depth + BayDist + UrbanDist + Area + OpenLevee + Island + DistLandfill +} \\
\hline DepthSD & 13 & 24575.4 & 24601.5 & 3.56 & 0.02 & 5.93 \\
\hline \multicolumn{7}{|l|}{ Depth + BayDist + UrbanDist + Area + OpenLevee + HuntLevee + Island +} \\
\hline DistLandfill + DepthSD & 14 & 24573.4 & 24601.5 & 3.58 & 0.02 & 5.99 \\
\hline \multicolumn{7}{|l|}{ Depth + Salinity + BayDist + UrbanDist + Area + OpenLevee + HuntLevee } \\
\hline + Island + DistLandfill + Breached & 15 & 24571.6 & 24601.8 & 3.80 & 0.01 & 6.69 \\
\hline Depth + BayDist + Area + OpenLevee + HuntLevee + Island + DistLandfill & 12 & 24577.8 & 24601.9 & 3.94 & 0.01 & 7.18 \\
\hline
\end{tabular}




\begin{tabular}{|c|c|c|c|c|c|c|}
\hline Model name & k & $-2 \log L$ & $\mathrm{AlCc}$ & $\triangle \mathrm{AICc}$ & $\mathbf{w i}_{\mathbf{i}}$ & $\begin{array}{l}\text { Evidence } \\
\text { ratio }\end{array}$ \\
\hline \multicolumn{7}{|c|}{ Depth + Salinity + BayDist + Area + OpenLevee + HuntLevee + Island +} \\
\hline DistLandfill & 13 & 24575.8 & 24601.9 & 3.96 & 0.01 & 7.24 \\
\hline \multicolumn{7}{|c|}{ Depth + Salinity + BayDist + UrbanDist + Area + OpenLevee + Island +} \\
\hline DistLandfill + Breached & 14 & 24573.8 & 24601.9 & 3.98 & 0.01 & 7.31 \\
\hline \multicolumn{7}{|c|}{ Depth + Salinity + BayDist + UrbanDist + Area + OpenLevee + Island +} \\
\hline DistLandfill + DepthSD & 14 & 24573.8 & 24601.9 & 3.98 & 0.01 & 7.31 \\
\hline Null Model & 5 & 24683.0 & 24693.0 & 95.05 & 2.19E-22 & $4.37 \mathrm{E}+20$ \\
\hline
\end{tabular}


Table 17. The ranking of candidate models at the pond scale for foraging eared grebes in the former salt production ponds of South San Francisco Bay, California.

[Models are ranked by differences in Akaike's information criterion and only models of $\triangle \mathrm{AIC}<4$ and the null model are presented. Birds were surveyed across the former salt ponds during October through April 2002-2015. Variables considered included the mean pond depth (Depth), the presence of islands (Island), variation in topography (DepthSD), \% of pond levee open to hunting (HuntLevee), \% of pond levee open to the public (OpenLevee), pond area (Area), water salinity, pond distance to urban areas (UrbanDist), and pond distance to the bay (BayDist). k: Number of estimated parameters in the model. AICc: Second-order Akaike's information criterion. $\triangle \mathrm{AICc}$ : The difference between the AICc of the current model and the AICc of the top model. wi: Akaike weight-likelihood of the model relative to candidate models. Evidence ratio: The weight of evidence that the current model is inferior to the top model]

\begin{tabular}{|c|c|c|c|c|c|c|}
\hline Model name & k & $-2 \log L$ & $\mathrm{AICc}$ & $\triangle \mathrm{AICc}$ & Wi & $\begin{array}{c}\text { Evidence } \\
\text { ratio }\end{array}$ \\
\hline Depth + Salinity + Area + OpenLevee + Breached & 10 & 13733.1 & 13753.2 & 0.00 & 0.04 & 1.00 \\
\hline Depth + Salinity + BayDist + Area + Island + Breached & 11 & 13731.4 & 13753.5 & 0.34 & 0.04 & 1.18 \\
\hline Depth + Salinity + Area + OpenLevee + Island + Breached & 11 & 13731.5 & 13753.6 & 0.38 & 0.04 & 1.21 \\
\hline Depth + Salinity + BayDist + Area + OpenLevee + Island + Breached & 12 & 13729.5 & 13753.6 & 0.41 & 0.04 & 1.23 \\
\hline Depth + Salinity + BayDist + Area + OpenLevee + Breached & 11 & 13731.6 & 13753.7 & 0.50 & 0.03 & 1.28 \\
\hline Depth + Salinity + BayDist + Area + Breached & 10 & 13733.7 & 13753.7 & 0.56 & 0.03 & 1.32 \\
\hline Depth + Salinity + Area + Breached & 9 & 13736.0 & 13754.0 & 0.87 & 0.03 & 1.54 \\
\hline Depth + Salinity + BayDist + UrbanDist + Area + Island + Breached & 12 & 13730.0 & 13754.2 & 0.97 & 0.03 & 1.63 \\
\hline Depth + Salinity + Area + Island + Breached & 10 & 13734.3 & 13754.3 & 1.16 & 0.02 & 1.79 \\
\hline \multicolumn{7}{|l|}{ Depth + Salinity + BayDist + UrbanDist + Area + OpenLevee + Island +} \\
\hline Breached & 13 & 13728.4 & 13754.5 & 1.31 & 0.02 & 1.93 \\
\hline Depth + Salinity + Area + OpenLevee + HuntLevee + Breached & 11 & 13732.4 & 13754.5 & 1.34 & 0.02 & 1.95 \\
\hline Depth + Salinity + BayDist + UrbanDist + Area + Breached & 11 & 13732.4 & 13754.5 & 1.36 & 0.02 & 1.97 \\
\hline Depth + Salinity + Area + Breached + DepthSD & 10 & 13734.5 & 13754.6 & 1.38 & 0.02 & 1.99 \\
\hline Depth + Salinity + UrbanDist + Area + OpenLevee + Breached & 11 & 13732.5 & 13754.6 & 1.40 & 0.02 & 2.01 \\
\hline Depth + Salinity + BayDist + Area + HuntLevee + Island + Breached & 12 & 13730.5 & 13754.6 & 1.45 & 0.02 & 2.07 \\
\hline Depth + Salinity + BayDist + UrbanDist + Area + OpenLevee + Breached & 12 & 13730.6 & 13754.7 & 1.53 & 0.02 & 2.15 \\
\hline Depth + Salinity + BayDist + Area + HuntLevee + Breached & 11 & 13732.7 & 13754.6 & 1.58 & 0.02 & 2.20 \\
\hline Depth + Salinity + Area + OpenLevee + Breached + DepthSD & 11 & 13732.8 & 13754.9 & 1.68 & 0.02 & 2.31 \\
\hline Depth + Salinity + UrbanDist + Area + OpenLevee + Island + Breached & 12 & 13730.8 & 13754.9 & 1.71 & 0.02 & 2.35 \\
\hline Depth + Salinity + Area + OpenLevee + HuntLevee + Island + Breached & 12 & 13730.8 & 13755.0 & 1.77 & 0.02 & 2.43 \\
\hline \multicolumn{7}{|l|}{ Depth + Salinity + BayDist + Area + OpenLevee + HuntLevee + Island +} \\
\hline Breached & 13 & 13728.8 & 13755.0 & 1.79 & 0.02 & 2.45 \\
\hline Depth + Salinity + BayDist + Area + OpenLevee + HuntLevee + Breached & 12 & 13730.9 & 13755.0 & 1.81 & 0.02 & 2.47 \\
\hline Depth + Salinity + Area + Island + Breached + DepthSD & 11 & 13732.9 & 13755.0 & 1.82 & 0.02 & 2.48 \\
\hline Depth + Salinity + Area + HuntLevee + Breached & 10 & 13734.9 & 13755.0 & 1.84 & 0.02 & 2.51 \\
\hline Depth + Salinity + BayDist + Area + Island + Breached + DepthSD & 12 & 13731.2 & 13755.3 & 2.09 & 0.02 & 2.85 \\
\hline Depth + Salinity + UrbanDist + Area + Breached & 10 & 13735.2 & 13755.3 & 2.10 & 0.02 & 2.86 \\
\hline Depth + Salinity + Area + OpenLevee + Island + Breached + DepthSD & 12 & 13731.2 & 13755.3 & 2.11 & 0.02 & 2.88 \\
\hline \multicolumn{7}{|l|}{ Depth + Salinity + BayDist + UrbanDist + Area + HuntLevee + Island +} \\
\hline Breached & 13 & 13729.2 & 13755.3 & 2.13 & 0.02 & 2.90 \\
\hline Depth + Salinity + BayDist + Area + Breached + DepthSD & 11 & 13733.2 & 13755.3 & 2.14 & 0.02 & 2.91 \\
\hline Depth + Salinity + Area + HuntLevee + Island + Breached & 11 & 13733.3 & 13755.4 & 2.24 & 0.01 & 3.06 \\
\hline Depth + Salinity + UrbanDist + Area + Island + Breached & 11 & 13733.4 & 13755.51 & 2.34 & 0.01 & 3.21 \\
\hline Depth + Salinity + BayDist + UrbanDist + Area + HuntLevee + Breached & 12 & 13731.5 & 13755.6 & 2.41 & 0.01 & 3.34 \\
\hline \multicolumn{7}{|l|}{ Depth + Salinity + BayDist + Area + OpenLevee + Island + Breached +} \\
\hline DepthSD & 13 & 13729.5 & 13755.6 & 2.43 & 0.01 & 3.37 \\
\hline Depth + Salinity + BayDist + Area + OpenLevee + Breached + DepthSD & 12 & 13731.6 & 13755.7 & 2.49 & 0.01 & 3.48 \\
\hline
\end{tabular}




\begin{tabular}{|c|c|c|c|c|c|c|}
\hline Model name & k & $-2 \log L$ & AICc & $\triangle \mathrm{AICc}$ & $\mathbf{W i}_{\mathrm{i}}$ & $\begin{array}{c}\text { Evidence } \\
\text { ratio }\end{array}$ \\
\hline Depth + Salinity + UrbanDist + Area + Breached + DepthSD & 11 & 13733.6 & 13755.7 & 2.52 & 0.01 & 3.52 \\
\hline Depth + Salinity + Area + HuntLevee + Breached + DepthSD & 11 & 13733.6 & 13755.7 & 2.54 & 0.01 & 3.55 \\
\hline \multicolumn{7}{|l|}{ Depth + Salinity + BayDist + UrbanDist + Area + OpenLevee + HuntLevee } \\
\hline+ Island + Breached & 14 & 13727.7 & 13755.9 & 2.71 & 0.01 & 3.88 \\
\hline \multicolumn{7}{|l|}{ Depth + Salinity + BayDist + UrbanDist + Area + Island + Breached + } \\
\hline DepthSD & 13 & 13729.8 & 13755.9 & 2.73 & 0.01 & 3.92 \\
\hline \multicolumn{7}{|l|}{ Depth + Salinity + UrbanDist + Area + OpenLevee + HuntLevee +} \\
\hline Breached & 12 & 13731.8 & 13755.9 & 2.73 & 0.01 & 3.92 \\
\hline \multicolumn{7}{|l|}{ Depth + Salinity + BayDist + UrbanDist + Area + OpenLevee + HuntLevee } \\
\hline+ Breached & 13 & 13729.9 & 13756.0 & 2.85 & 0.01 & 4.16 \\
\hline Depth + Salinity + UrbanDist + Area + Island + Breached + DepthSD & 12 & 13732.0 & 13756.1 & 2.89 & 0.01 & 4.25 \\
\hline Depth + Salinity + BayDist + UrbanDist + Area + Breached + DepthSD & 12 & 13732.0 & 13756.1 & 2.95 & 0.01 & 4.38 \\
\hline Depth + Salinity + UrbanDist + Area + OpenLevee + Breached + DepthSD & 12 & 13732.1 & 13756.2 & 2.99 & 0.01 & 4.46 \\
\hline Depth + Salinity + Area + OpenLevee + HuntLevee + Breached + DepthSD & 12 & 13732.1 & 13756.2 & 3.03 & 0.01 & 4.55 \\
\hline Depth + Salinity + Area + HuntLevee + Island + Breached + DepthSD & 12 & 13732.1 & 13756.2 & 3.05 & 0.01 & 4.60 \\
\hline \multicolumn{7}{|l|}{ Depth + Salinity + UrbanDist + Area + OpenLevee + HuntLevee + Island +} \\
\hline Breached & 13 & 13730.2 & 13756.3 & 3.11 & 0.01 & 4.74 \\
\hline Depth + Salinity + UrbanDist + Area + HuntLevee + Breached & 11 & 13734.2 & 13756.3 & 3.12 & 0.01 & 4.75 \\
\hline Depth + Salinity + BayDist + Area + HuntLevee + Breached + DepthSD & 12 & 13732.3 & 13756.4 & 3.25 & 0.01 & 5.08 \\
\hline \multicolumn{7}{|l|}{ Depth + Salinity + BayDist + Area + HuntLevee + Island + Breached +} \\
\hline DepthSD & 13 & 13730.3 & 13756.5 & 3.27 & 0.01 & 5.13 \\
\hline \multicolumn{7}{|l|}{ Depth + Salinity + BayDist + UrbanDist + Area + OpenLevee + Island +} \\
\hline Breached + DepthSD & 14 & 13728.4 & 13756.5 & 3.33 & 0.01 & 5.29 \\
\hline \multicolumn{7}{|l|}{ Depth + Salinity + UrbanDist + Area + OpenLevee + Island + Breached +} \\
\hline DepthSD & 13 & 13730.4 & 13756.6 & 3.37 & 0.01 & 5.39 \\
\hline Depth + Salinity + UrbanDist + Area + HuntLevee + Island + Breached & 12 & 13732.5 & 13756.6 & 3.45 & 0.01 & 5.62 \\
\hline \multicolumn{7}{|l|}{ Depth + Salinity + BayDist + UrbanDist + Area + OpenLevee + Breached +} \\
\hline DepthSD & 13 & 13730.6 & 13756.7 & 3.53 & 0.01 & 5.84 \\
\hline \multicolumn{7}{|l|}{ Depth + Salinity + Area + OpenLevee + HuntLevee + Island + Breached +} \\
\hline DepthSD & 13 & 13730.6 & 13756.7 & 3.53 & 0.01 & 5.84 \\
\hline Depth + Salinity + UrbanDist + Area + HuntLevee + Breached + DepthSD & 12 & 13732.8 & 13756.9 & 3.71 & 0.01 & 6.40 \\
\hline \multicolumn{7}{|l|}{ Depth + Salinity + BayDist + Area + OpenLevee + HuntLevee + Island +} \\
\hline Breached + DepthSD & 14 & 13728.8 & 13757.0 & 3.81 & 0.01 & 6.72 \\
\hline \multicolumn{7}{|l|}{ Depth + Salinity + BayDist + Area + OpenLevee + HuntLevee + Breached +} \\
\hline DepthSD & 13 & 13730.7 & 13757.0 & 3.81 & 0.01 & 6.72 \\
\hline \multicolumn{7}{|l|}{ Depth + Salinity + BayDist + UrbanDist + Area + HuntLevee + Island +} \\
\hline Breached + DepthSD & 14 & 13729.0 & 13757.2 & 3.97 & 0.01 & 7.28 \\
\hline Null Model & 5 & 14023.5 & 14033.5 & 280.30 & $6.01 \mathrm{E}-63$ & $7.37 \mathrm{E}+60$ \\
\hline
\end{tabular}


Table 18. The ranking of candidate models at the pond scale for roosting eared grebes in the former salt production ponds of South San Francisco Bay, California.

[Models are ranked by differences in Akaike's information criterion and only models of $\triangle \mathrm{AIC}<4$ and the null model are presented. Birds were surveyed across the former salt ponds during October through April 2002-2015. Variables considered included the mean pond depth (Depth), the presence of islands (Island), variation in topography (DepthSD), \% of pond levee open to hunting (HuntLevee), \% of pond levee open to the public (OpenLevee), pond area (Area), water salinity, pond distance to urban areas (UrbanDist), and pond distance to the bay (BayDist). k: Number of estimated parameters in the model. AICc: Second-order Akaike's information criterion. $\triangle$ AICc: The difference between the AICc of the current model and the AICc of the top model. wi: Akaike weight-likelihood of the model relative to candidate models. Evidence ratio: The weight of evidence that the current model is inferior to the top model]

\begin{tabular}{|c|c|c|c|c|c|c|}
\hline Model name & k & $-2 \log L$ & AICc & $\triangle \mathrm{AICc}$ & $\mathbf{w}_{\mathrm{i}}$ & $\begin{array}{c}\text { Evidence } \\
\text { ratio }\end{array}$ \\
\hline Depth + BayDist + PondArea + Breached & 9 & 11686.2 & 11704.2 & 0.00 & 0.07 & 1.00 \\
\hline Depth + Salinity + BayDist + PondArea + Breached & 10 & 11685.3 & 11705.4 & 1.17 & 0.04 & 1.80 \\
\hline Depth + BayDist + PondArea + OpenLevee + Breached & 10 & 11685.3 & 11705.4 & 1.17 & 0.04 & 1.80 \\
\hline Depth + BayDist + PondArea + Island + Breached & 10 & 11685.8 & 11705.9 & 1.67 & 0.03 & 2.31 \\
\hline Depth + BayDist + PondArea + HuntLevee + Breached & 10 & 11686.0 & 11706.1 & 1.85 & 0.03 & 2.53 \\
\hline Depth + PondArea + Breached & 8 & 11690.0 & 11706.1 & 1.87 & 0.03 & 2.54 \\
\hline Depth + BayDist + UrbanDist + PondArea + Breached & 10 & 11686.1 & 11706.1 & 1.91 & 0.03 & 2.60 \\
\hline Depth + BayDist + PondArea + Breached + DepthSD & 10 & 11686.1 & 11706.2 & 1.95 & 0.03 & 2.66 \\
\hline Depth + Salinity + BayDist + PondArea + OpenLevee + Breached & 11 & 11684.4 & 11706.5 & 2.29 & 0.02 & 3.14 \\
\hline Depth + PondArea + OpenLevee + Breached & 9 & 11688.5 & 11706.6 & 2.38 & 0.02 & 3.29 \\
\hline Depth + Salinity + PondArea + Breached & 9 & 11688.6 & 11706.6 & 2.40 & 0.02 & 3.32 \\
\hline Depth + Salinity + BayDist + PondArea + Island + Breached & 11 & 11684.8 & 11706.9 & 2.69 & 0.02 & 3.84 \\
\hline Depth + BayDist + PondArea + OpenLevee + Breached + DepthSD & 11 & 11684.9 & 11707.0 & 2.77 & 0.02 & 3.99 \\
\hline Depth + BayDist + PondArea + OpenLevee + Island + Breached & 11 & 11685.0 & 11707.1 & 2.87 & 0.02 & 4.20 \\
\hline Depth + Salinity + PondArea + OpenLevee + Breached & 10 & 11687.0 & 11707.1 & 2.87 & 0.02 & 4.21 \\
\hline Depth + BayDist + PondArea + OpenLevee + HuntLevee + Breached & 11 & 11685.1 & 11707.2 & 2.95 & 0.02 & 4.37 \\
\hline Depth + Salinity + BayDist + UrbanDist + PondArea + Breached & 11 & 11685.2 & 11707.3 & 3.05 & 0.02 & 4.59 \\
\hline Depth + Salinity + BayDist + PondArea + HuntLevee + Breached & 11 & 11685.2 & 11707.3 & 3.05 & 0.02 & 4.59 \\
\hline Depth + BayDist + UrbanDist + PondArea + OpenLevee + Breached & 11 & 11685.3 & 11707.4 & 3.13 & 0.01 & 4.78 \\
\hline Depth + Salinity + BayDist + PondArea + Breached + DepthSD & 11 & 11685.3 & 11707.4 & 3.15 & 0.01 & 4.83 \\
\hline Depth + BayDist + PondArea + HuntLevee + Island + Breached & 11 & 11685.7 & 11707.8 & 3.53 & 0.01 & 5.84 \\
\hline Depth + PondArea + Breached + DepthSD & 9 & 11689.7 & 11707.8 & 3.56 & 0.01 & 5.93 \\
\hline Depth + BayDist + UrbanDist + PondArea + Island + Breached & 11 & 11685.7 & 11707.8 & 3.57 & 0.01 & 5.96 \\
\hline Depth + BayDist + PondArea + Island + Breached + DepthSD & 11 & 11685.7 & 11707.8 & 3.59 & 0.01 & 6.02 \\
\hline Depth + PondArea + Island + Breached & 9 & 11689.9 & 11708.0 & 3.76 & 0.01 & 6.55 \\
\hline Depth + BayDist + UrbanDist + PondArea + HuntLevee + Breached & 11 & 11685.9 & 11708.0 & 3.77 & 0.01 & 6.58 \\
\hline Depth + BayDist + PondArea + HuntLevee + Breached + DepthSD & 11 & 11685.9 & 11708.0 & 3.81 & 0.01 & 6.72 \\
\hline Depth + Salinity + BayDist + PondArea + OpenLevee + Island + Breached & 12 & 11684.0 & 11708.1 & 3.85 & 0.01 & 6.84 \\
\hline Depth + BayDist + UrbanDist + PondArea + Breached + DepthSD & 11 & 11686.0 & 11708.1 & 3.85 & 0.01 & 6.85 \\
\hline Depth + UrbanDist + PondArea + Breached & 9 & 11690.0 & 11708.1 & 3.86 & 0.01 & 6.89 \\
\hline \multicolumn{7}{|l|}{ Depth + Salinity + BayDist + PondArea + OpenLevee + Breached +} \\
\hline DepthSD & 12 & 11684.0 & 11708.1 & 3.91 & 0.01 & 7.05 \\
\hline Null Model & 5 & 12015.5 & 12025.5 & 321.26 & $1.24 \mathrm{E}-71$ & $5.76 \mathrm{E}+69$ \\
\hline
\end{tabular}


Table 19. The ranking of candidate models at the pond scale for foraging northern shovelers in the former salt production ponds of South San Francisco Bay, California.

[Models are ranked by differences in Akaike's information criterion and only models of $\triangle \mathrm{AIC}<4$ and the null model are presented. Birds were surveyed across the former salt ponds during October through April 2002-2015. Variables considered included the mean pond depth (Depth), the presence of islands (Island), variation in topography (DepthSD), \% of pond levee open to hunting (HuntLevee), \% of pond levee open to the public (OpenLevee), pond area (Area), water salinity, pond distance to urban areas (UrbanDist), and pond distance to the bay (BayDist). k: Number of estimated parameters in the model. AICc: Second-order Akaike's information criterion. $\triangle$ AICc: The difference between the AICc of the current model and the AICc of the top model. wi: Akaike weight-likelihood of the model relative to candidate models. Evidence ratio: The weight of evidence that the current model is inferior to the top model]

\begin{tabular}{|c|c|c|c|c|c|c|}
\hline Model name & k & $-2 \log L$ & AlCc & $\triangle \mathrm{AICc}$ & $\mathbf{w}_{\mathrm{i}}$ & $\begin{array}{c}\text { Evidence } \\
\text { ratio }\end{array}$ \\
\hline Salinity + Island + DepthSD & 8 & 18522.6 & 18538.6 & 0.00 & 0.03 & 1.00 \\
\hline Salinity + OpenLevee + Island & 8 & 18522.7 & 18538.7 & 0.10 & 0.03 & 1.05 \\
\hline Salinity + OpenLevee + Island + DepthSD & 9 & 18521.3 & 18539.4 & 0.73 & 0.02 & 1.44 \\
\hline Salinity + Area + OpenLevee + Island & 9 & 18521.4 & 18539.5 & 0.87 & 0.02 & 1.55 \\
\hline Depth + Salinity + Island + DepthSD & 9 & 18521.6 & 18539.6 & 0.99 & 0.02 & 1.64 \\
\hline Salinity + HuntLevee + Island + DepthSD & 9 & 18521.6 & 18539.6 & 0.99 & 0.02 & 1.64 \\
\hline Salinity + OpenLevee + HuntLevee + Island & 9 & 18521.6 & 18539.6 & 1.01 & 0.02 & 1.66 \\
\hline Salinity + Area + Island + DepthSD & 9 & 18521.7 & 18539.8 & 1.17 & 0.01 & 1.80 \\
\hline Salinity + Island & 7 & 18526.0 & 18540.0 & 1.39 & 0.01 & 2.00 \\
\hline Salinity + UrbanDist + Island + DepthSD & 9 & 18522.0 & 18540.0 & 1.41 & 0.01 & 2.03 \\
\hline Depth + Salinity + OpenLevee + Island & 9 & 18522.1 & 18540.1 & 1.51 & 0.01 & 2.13 \\
\hline Salinity + OpenLevee + HuntLevee + Island + DepthSD & 10 & 18520.1 & 18540.2 & 1.57 & 0.01 & 2.19 \\
\hline Depth + Salinity + OpenLevee + Island + DepthSD & 10 & 18520.2 & 18540.2 & 1.61 & 0.01 & 2.23 \\
\hline Salinity + Island + Breached + DepthSD & 9 & 18522.2 & 18540.3 & 1.63 & 0.01 & 2.26 \\
\hline Salinity + UrbanDist + OpenLevee + Island & 9 & 18522.3 & 18540.3 & 1.71 & 0.01 & 2.35 \\
\hline Salinity + Area + OpenLevee + HuntLevee + Island & 10 & 18520.3 & 18540.4 & 1.73 & 0.01 & 2.37 \\
\hline Salinity + Area + OpenLevee + Island + DepthSD & 10 & 18520.3 & 18540.4 & 1.75 & 0.01 & 2.39 \\
\hline Salinity + BayDist + OpenLevee + Island & 9 & 18522.3 & 18540.4 & 1.75 & 0.01 & 2.40 \\
\hline Salinity + BayDist + Island + DepthSD & 9 & 18522.6 & 18540.6 & 1.97 & 0.01 & 2.68 \\
\hline Depth + Salinity + HuntLevee + Island + DepthSD & 10 & 18520.6 & 18540.6 & 2.01 & 0.01 & 2.73 \\
\hline Salinity + OpenLevee + Island + Breached & 9 & 18522.6 & 18540.6 & 2.01 & 0.01 & 2.74 \\
\hline Depth + Salinity + Area + Island + DepthSD & 10 & 18520.7 & 18540.7 & 2.11 & 0.01 & 2.87 \\
\hline Salinity + Area + HuntLevee + Island + DepthSD & 10 & 18520.7 & 18540.8 & 2.15 & 0.01 & 2.92 \\
\hline Depth + Salinity + Area + OpenLevee + Island & 10 & 18520.7 & 18540.8 & 2.19 & 0.01 & 2.98 \\
\hline Depth + Salinity + UrbanDist + Island + DepthSD & 10 & 18520.8 & 18540.9 & 2.27 & 0.01 & 3.11 \\
\hline Salinity + Area + Island & 8 & 18524.9 & 18540.9 & 2.28 & 0.01 & 3.13 \\
\hline Salinity + UrbanDist + OpenLevee + Island + DepthSD & 10 & 18520.8 & 18540.9 & 2.29 & 0.01 & 3.14 \\
\hline Salinity + UrbanDist + HuntLevee + Island + DepthSD & 10 & 18520.9 & 18541.0 & 2.37 & 0.01 & 3.26 \\
\hline Depth + Salinity + OpenLevee + HuntLevee + Island & 10 & 18521.0 & 18541.1 & 2.45 & 0.01 & 3.40 \\
\hline Depth + Salinity + OpenLevee + HuntLevee + Island + DepthSD & 11 & 18519.0 & 18541.1 & 2.46 & 0.01 & 3.42 \\
\hline Salinity + BayDist + Island & 8 & 18525.1 & 18541.2 & 2.52 & 0.01 & 3.53 \\
\hline Depth + Salinity + Area + OpenLevee + Island + DepthSD & 11 & 18519.1 & 18541.2 & 2.54 & 0.01 & 3.56 \\
\hline Salinity + OpenLevee + Island + Breached + DepthSD & 10 & 18521.1 & 18541.2 & 2.55 & 0.01 & 3.57 \\
\hline Salinity + Area + OpenLevee + HuntLevee + Island + DepthSD & 11 & 18519.1 & 18541.2 & 2.56 & 0.01 & 3.60 \\
\hline Salinity + UrbanDist + OpenLevee + HuntLevee + Island & 10 & 18521.1 & 18541.2 & 2.59 & 0.01 & 3.64 \\
\hline Salinity + HuntLevee + Island & 8 & 18525.2 & 18541.2 & 2.60 & 0.01 & 3.67 \\
\hline Salinity + HuntLevee + Island + Breached + DepthSD & 10 & 18521.2 & 18541.2 & 2.61 & 0.01 & 3.68 \\
\hline Salinity + BayDist + Area + OpenLevee + Island & 10 & 18521.2 & 18541.3 & 2.63 & 0.01 & 3.72 \\
\hline
\end{tabular}




\begin{tabular}{|c|c|c|c|c|c|c|}
\hline Model name & k & $-2 \log L$ & AICc & $\triangle \mathrm{AICc}$ & $\mathbf{w}_{\mathrm{i}}$ & $\begin{array}{c}\text { Evidence } \\
\text { ratio }\end{array}$ \\
\hline Salinity + BayDist + OpenLevee + HuntLevee + Island & 10 & 18521.2 & 18541.3 & 2.65 & 0.01 & 3.76 \\
\hline Salinity + BayDist + OpenLevee + Island + DepthSD & 10 & 18521.3 & 18541.3 & 2.71 & 0.01 & 3.87 \\
\hline Salinity + UrbanDist + Area + OpenLevee + Island & 10 & 18521.3 & 18541.4 & 2.75 & 0.01 & 3.95 \\
\hline Depth + Salinity + Island + Breached + DepthSD & 10 & 18521.3 & 18541.4 & 2.79 & 0.01 & 4.03 \\
\hline Salinity + Area + OpenLevee + Island + Breached & 10 & 18521.4 & 18541.4 & 2.81 & 0.01 & 4.07 \\
\hline Salinity + UrbanDist + Island & 8 & 18525.4 & 18541.5 & 2.84 & 0.01 & 4.14 \\
\hline Salinity + UrbanDist + Area + Island + DepthSD & 10 & 18521.4 & 18541.5 & 2.87 & 0.01 & 4.19 \\
\hline Salinity + Area + Island + Breached + DepthSD & 10 & 18521.4 & 18541.5 & 2.87 & 0.01 & 4.19 \\
\hline Depth + Salinity + BayDist + Island + DepthSD & 10 & 18521.5 & 18541.6 & 2.93 & 0.01 & 4.32 \\
\hline Salinity + OpenLevee + HuntLevee + Island + Breached & 10 & 18521.5 & 18541.6 & 2.93 & 0.01 & 4.32 \\
\hline Depth + Salinity + BayDist + OpenLevee + Island & 10 & 18521.5 & 18541.6 & 2.95 & 0.01 & 4.36 \\
\hline Salinity + BayDist + HuntLevee + Island + DepthSD & 10 & 18521.5 & 18541.6 & 2.97 & 0.01 & 4.41 \\
\hline Depth + Salinity + UrbanDist + OpenLevee + Island + DepthSD & 11 & 18519.6 & 18541.7 & 3.02 & 0.01 & 4.53 \\
\hline Salinity + UrbanDist + Island + Breached + DepthSD & 10 & 18521.6 & 18541.7 & 3.05 & 0.01 & 4.59 \\
\hline Depth + Salinity + UrbanDist + OpenLevee + Island & 10 & 18521.6 & 18541.7 & 3.07 & 0.01 & 4.63 \\
\hline Salinity + Island + Breached & 8 & 18525.7 & 18541.7 & 3.08 & 0.01 & 4.66 \\
\hline Depth + Salinity + Area + OpenLevee + HuntLevee + Island & 11 & 18519.6 & 18541.7 & 3.08 & 0.01 & 4.67 \\
\hline Salinity + UrbanDist + OpenLevee + HuntLevee + Island + DepthSD & 11 & 18519.6 & 18541.7 & 3.08 & 0.01 & 4.67 \\
\hline Depth + Salinity + Area + HuntLevee + Island + DepthSD & 11 & 18519.6 & 18541.7 & 3.10 & 0.01 & 4.72 \\
\hline Depth + Salinity + Island & 8 & 18525.7 & 18541.8 & 3.14 & 0.01 & 4.81 \\
\hline Salinity + BayDist + Area + Island + DepthSD & 10 & 18521.7 & 18541.8 & 3.17 & 0.01 & 4.87 \\
\hline Depth + Salinity + UrbanDist + HuntLevee + Island + DepthSD & 11 & 18519.8 & 18541.9 & 3.24 & 0.01 & 5.06 \\
\hline Salinity + BayDist + UrbanDist + OpenLevee + Island & 10 & 18521.8 & 18541.9 & 3.25 & 0.01 & 5.07 \\
\hline Salinity + BayDist + UrbanDist + Island + DepthSD & 10 & 18521.9 & 18542.0 & 3.33 & 0.01 & 5.28 \\
\hline Depth + Salinity + Area + OpenLevee + HuntLevee + Island + DepthSD & 12 & 18517.9 & 18542.0 & 3.38 & 0.00 & 5.42 \\
\hline Salinity + OpenLevee + HuntLevee + Island + Breached + DepthSD & 11 & 18519.9 & 18542.1 & 3.38 & 0.00 & 5.42 \\
\hline Salinity + Area + HuntLevee + Island & 9 & 18524.0 & 18542.1 & 3.47 & 0.00 & 5.68 \\
\hline Salinity + BayDist + Area + OpenLevee + HuntLevee + Island & 11 & 18520.0 & 18542.1 & 3.48 & 0.00 & 5.70 \\
\hline Depth + Salinity + OpenLevee + Island + Breached & 10 & 18522.1 & 18542.1 & 3.51 & 0.00 & 5.77 \\
\hline Depth + Salinity + BayDist + OpenLevee + Island + DepthSD & 11 & 18520.1 & 18542.2 & 3.54 & 0.00 & 5.88 \\
\hline Salinity + BayDist + OpenLevee + HuntLevee + Island + DepthSD & 11 & 18520.1 & 18542.2 & 3.54 & 0.00 & 5.88 \\
\hline Salinity + BayDist + Area + Island & 9 & 18524.1 & 18542.2 & 3.55 & 0.00 & 5.91 \\
\hline Depth + Salinity + OpenLevee + Island + Breached + DepthSD & 11 & 18520.1 & 18542.2 & 3.56 & 0.00 & 5.94 \\
\hline Salinity + UrbanDist + Area + OpenLevee + Island + DepthSD & 11 & 18520.1 & 18542.2 & 3.56 & 0.00 & 5.94 \\
\hline Salinity + UrbanDist + Area + OpenLevee + HuntLevee + Island & 11 & 18520.1 & 18542.2 & 3.58 & 0.00 & 6.00 \\
\hline Salinity + BayDist + Island + Breached + DepthSD & 10 & 18522.1 & 18542.2 & 3.59 & 0.00 & 6.01 \\
\hline Salinity + BayDist + OpenLevee + Island + Breached & 10 & 18522.2 & 18542.2 & 3.61 & 0.00 & 6.07 \\
\hline Salinity + Area + OpenLevee + Island + Breached + DepthSD & 11 & 18520.2 & 18542.3 & 3.62 & 0.00 & 6.12 \\
\hline Salinity + UrbanDist + OpenLevee + Island + Breached & 10 & 18522.2 & 18542.3 & 3.63 & 0.00 & 6.13 \\
\hline Depth + Salinity + UrbanDist + Area + Island + DepthSD & 11 & 18520.2 & 18542.3 & 3.68 & 0.00 & 6.30 \\
\hline Salinity + Area + OpenLevee + HuntLevee + Island + Breached & 11 & 18520.2 & 18542.3 & 3.68 & 0.00 & 6.30 \\
\hline Salinity + BayDist + HuntLevee + Island & 9 & 18524.3 & 18542.3 & 3.69 & 0.00 & 6.34 \\
\hline Depth + Salinity + BayDist + Area + OpenLevee + Island & 11 & 18520.3 & 18542.4 & 3.72 & 0.00 & 6.43 \\
\hline Salinity + BayDist + Area + OpenLevee + Island + DepthSD & 11 & 18520.3 & 18542.4 & 3.74 & 0.00 & 6.49 \\
\hline Salinity + BayDist + UrbanDist + Island & 9 & 18524.3 & 18542.4 & 3.77 & 0.00 & 6.59 \\
\hline Depth + Salinity + HuntLevee + Island + Breached + DepthSD & 11 & 18520.3 & 18542.4 & 3.78 & 0.00 & 6.63 \\
\hline Salinity + UrbanDist + Area + HuntLevee + Island + DepthSD & 11 & 18520.3 & 18542.4 & 3.80 & 0.00 & 6.69 \\
\hline
\end{tabular}




\begin{tabular}{|c|c|c|c|c|c|c|}
\hline Model name & k & $-2 \log L$ & AICc & $\triangle \mathrm{AICc}$ & $\mathbf{w}_{\mathrm{i}}$ & $\begin{array}{c}\text { Evidence } \\
\text { ratio }\end{array}$ \\
\hline Salinity + Area + HuntLevee + Island + Breached + DepthSD & 11 & 18520.4 & 18542.4 & 3.84 & 0.00 & 6.83 \\
\hline \multicolumn{7}{|l|}{ Depth + Salinity + UrbanDist + OpenLevee + HuntLevee + Island +} \\
\hline DepthSD & 12 & 18518.4 & 18542.5 & 3.86 & 0.00 & 6.88 \\
\hline Depth + Salinity + BayDist + OpenLevee + HuntLevee + Island & 11 & 18520.4 & 18542.5 & 3.88 & 0.00 & 6.97 \\
\hline Depth + Salinity + BayDist + HuntLevee + Island + DepthSD & 11 & 18520.5 & 18542.6 & 3.92 & 0.00 & 7.11 \\
\hline Depth + Salinity + Area + Island + Breached + DepthSD & 11 & 18520.5 & 18542.6 & 3.94 & 0.00 & 7.18 \\
\hline Depth + Salinity + UrbanDist + OpenLevee + HuntLevee + Island & 11 & 18520.5 & 18542.6 & 3.96 & 0.00 & 7.25 \\
\hline Salinity + UrbanDist + HuntLevee + Island + Breached + DepthSD & 11 & 18520.5 & 18542.6 & 3.98 & 0.00 & 7.32 \\
\hline Depth + Salinity + Area + Island & 9 & 18524.6 & 18542.6 & 3.99 & 0.00 & 7.36 \\
\hline Null Model & 5 & 18586.2 & 18596.2 & 57.61 & $8.21 \mathrm{E}-15$ & $3.24 \mathrm{E}+12$ \\
\hline
\end{tabular}


Table 20. The ranking of candidate models at the grid scale for roosting northern shovelers in the former salt production ponds of South San Francisco Bay, California.

[Models are ranked by differences in Akaike's information criterion and only models of $\triangle \mathrm{AIC}<4$ and the null model are presented. Birds were surveyed across the former salt ponds during October through April 2002-2015. Variables considered included the mean pond depth (Depth), the presence of islands (Island), variation in topography (DepthSD), \% of pond levee open to hunting (HuntLevee), \% of pond levee open to the public (OpenLevee), pond area (Area), water salinity, pond distance to urban areas (UrbanDist), and pond distance to the bay (BayDist). k: Number of estimated parameters in the model. AICc: Second-order Akaike's information criterion. $\triangle$ AICc: The difference between the AICc of the current model and the AICc of the top model. wi: Akaike weight-likelihood of the model relative to candidate models. Evidence ratio: The weight of evidence that the current model is inferior to the top model]

\begin{tabular}{|c|c|c|c|c|c|c|}
\hline Model name & k & $-2 \log L$ & AICc & $\triangle \mathrm{AICc}$ & $\mathbf{w}_{\mathrm{i}}$ & $\begin{array}{c}\text { Evidence } \\
\text { ratio }\end{array}$ \\
\hline Depth + Salinity + HuntLevee + Island + DepthSD & 10 & 21675.8 & 21695.9 & 0.00 & 0.07 & 1.00 \\
\hline Depth + Salinity + UrbanDist + HuntLevee + Island + DepthSD & 11 & 21673.8 & 21695.9 & 0.02 & 0.06 & 1.01 \\
\hline Depth + Salinity + PondArea + HuntLevee + Island + DepthSD & 11 & 21674.0 & 21696.1 & 0.22 & 0.06 & 1.11 \\
\hline Salinity + HuntLevee + Island + DepthSD & 9 & 21678.6 & 21696.7 & 0.79 & 0.04 & 1.48 \\
\hline Depth + Salinity + UrbanDist + PondArea + HuntLevee + Island + DepthSD & 12 & 21672.8 & 21696.9 & 1.03 & 0.04 & 1.68 \\
\hline Salinity + UrbanDist + HuntLevee + Island + DepthSD & 10 & 21677.0 & 21697.1 & 1.20 & 0.04 & 1.82 \\
\hline Salinity + PondArea + HuntLevee + Island + DepthSD & 10 & 21677.0 & 21697.1 & 1.20 & 0.04 & 1.82 \\
\hline Depth + Salinity + UrbanDist + HuntLevee + Island + Breached + DepthSD & 12 & 21673.4 & 21697.5 & 1.63 & 0.03 & 2.26 \\
\hline Depth + Salinity + PondArea + HuntLevee + Island + Breached + DepthSD & 12 & 21673.6 & 21697.7 & 1.83 & 0.03 & 2.50 \\
\hline Depth + Salinity + BayDist + HuntLevee + Island + DepthSD & 11 & 21675.8 & 21697.9 & 2.02 & 0.02 & 2.74 \\
\hline Depth + Salinity + OpenLevee + HuntLevee + Island + DepthSD & 11 & 21675.8 & 21697.9 & 2.02 & 0.02 & 2.74 \\
\hline Depth + Salinity + BayDist + UrbanDist + HuntLevee + Island + DepthSD & 12 & 21673.8 & 21697.9 & 2.03 & 0.02 & 2.76 \\
\hline Depth + Salinity + UrbanDist + OpenLevee + HuntLevee + Island + DepthSD & 12 & 21673.8 & 21697.9 & 2.03 & 0.02 & 2.76 \\
\hline Salinity + UrbanDist + PondArea + HuntLevee + Island + DepthSD & 11 & 21676.0 & 21698.1 & 2.22 & 0.02 & 3.03 \\
\hline Depth + Salinity + BayDist + PondArea + HuntLevee + Island + DepthSD & 12 & 21674.0 & 21698.1 & 2.23 & 0.02 & 3.05 \\
\hline Salinity + BayDist + HuntLevee + Island + DepthSD & 10 & 21678.4 & 21698.5 & 2.60 & 0.02 & 3.67 \\
\hline Salinity + OpenLevee + HuntLevee + Island + DepthSD & 10 & 21678.4 & 21698.5 & 2.60 & 0.02 & 3.67 \\
\hline \multicolumn{7}{|l|}{ Depth + Salinity + UrbanDist + PondArea + HuntLevee + Island + Breached } \\
\hline + DepthSD & 13 & 21672.4 & 21698.5 & 2.65 & 0.02 & 3.76 \\
\hline Salinity + HuntLevee + Island + Breached + DepthSD & 10 & 21678.6 & 21698.7 & 2.80 & 0.02 & 4.06 \\
\hline Salinity + BayDist + PondArea + HuntLevee + Island + DepthSD & 11 & 21676.8 & 21698.9 & 3.02 & 0.01 & 4.52 \\
\hline Salinity + UrbanDist + OpenLevee + HuntLevee + Island + DepthSD & 11 & 21676.8 & 21698.9 & 3.02 & 0.01 & 4.52 \\
\hline Salinity + PondArea + OpenLevee + HuntLevee + Island + DepthSD & 11 & 21676.8 & 21698.9 & 3.02 & 0.01 & 4.52 \\
\hline \multicolumn{7}{|l|}{ Depth + Salinity + UrbanDist + PondArea + OpenLevee + HuntLevee +} \\
\hline Island + DepthSD & 13 & 21672.8 & 21698.9 & 3.05 & 0.01 & 4.60 \\
\hline Salinity + BayDist + UrbanDist + HuntLevee + Island + DepthSD & 11 & 21677.0 & 21699.1 & 3.22 & 0.01 & 4.99 \\
\hline Salinity + UrbanDist + HuntLevee + Island + Breached + DepthSD & 11 & 21677.0 & 21699.1 & 3.22 & 0.01 & 4.99 \\
\hline Salinity + PondArea + HuntLevee + Island + Breached + DepthSD & 11 & 21677.0 & 21699.1 & 3.22 & 0.01 & 4.99 \\
\hline Depth + Salinity + OpenLevee + HuntLevee + Island + Breached + DepthSD & 12 & 21675.2 & 21699.3 & 3.43 & 0.01 & 5.56 \\
\hline \multicolumn{7}{|l|}{ Depth + Salinity + UrbanDist + OpenLevee + HuntLevee + Island +} \\
\hline Breached + DepthSD & 13 & 21673.2 & 21699.3 & 3.45 & 0.01 & 5.61 \\
\hline \multicolumn{7}{|l|}{ Depth + Salinity + PondArea + OpenLevee + HuntLevee + Island + Breached } \\
\hline + DepthSD & 13 & 21673.2 & 21699.3 & 3.45 & 0.01 & 5.61 \\
\hline Depth + Salinity + BayDist + HuntLevee + Island + Breached + DepthSD & 12 & 21675.4 & 21699.5 & 3.63 & 0.01 & 6.15 \\
\hline \multicolumn{7}{|l|}{ Depth + Salinity + BayDist + UrbanDist + HuntLevee + Island + Breached +} \\
\hline DepthSD & 13 & 21673.4 & 21699.5 & 3.65 & 0.01 & 6.20 \\
\hline \multicolumn{7}{|l|}{ Depth + Salinity + BayDist + PondArea + HuntLevee + Island + Breached +} \\
\hline DepthSD & 13 & 21673.4 & 21699.5 & 3.65 & 0.01 & 6.20 \\
\hline
\end{tabular}




\begin{tabular}{|c|c|c|c|c|c|c|}
\hline Model name & k & $-2 \log L$ & $\mathrm{AICc}$ & $\triangle \mathrm{AICc}$ & $w_{i}$ & $\begin{array}{c}\text { Evidence } \\
\text { ratio }\end{array}$ \\
\hline Depth + Salinity + BayDist + OpenLevee + HuntLevee + Island + DepthSD & 12 & 21675.6 & 21699.7 & 3.83 & 0.01 & 6.79 \\
\hline Null Model & 5 & 21841.2 & 21851.2 & 155.34 & $1.20 \mathrm{E}-35$ & $5.40 \mathrm{E}+33$ \\
\hline
\end{tabular}


Table 21. The ranking of candidate models at the pond scale for foraging and roosting ruddy ducks in the former salt production ponds of South San Francisco Bay, California.

[Models are ranked by differences in Akaike's information criterion and only models of $\triangle \mathrm{AIC}<4$ and the null model are presented. Birds were surveyed across the former salt ponds during October through April 2002-2015. Variables considered included the mean pond depth (Depth), the presence of islands (Island), variation in topography (DepthSD), \% of pond levee open to hunting (HuntLevee), \% of pond levee open to the public (OpenLevee), pond area (Area), water salinity, pond distance to urban areas (UrbanDist), and pond distance to the bay (BayDist). k: Number of estimated parameters in the model. AICc: Second-order Akaike's information criterion. $\triangle$ AICc: The difference between the AICc of the current model and the AICc of the top model. wi: Akaike weight-likelihood of the model relative to candidate models. Evidence ratio: The weight of evidence that the current model is inferior to the top model]

\begin{tabular}{|c|c|c|c|c|c|c|}
\hline Model name & k & $-2 \log L$ & AICc & $\triangle \mathrm{AICc}$ & $w_{i}$ & $\begin{array}{c}\text { Evidence } \\
\text { ratio }\end{array}$ \\
\hline \multicolumn{7}{|l|}{ Foraging } \\
\hline Depth + Salinity + BayDist + Area + Island + Breached & 11 & 14502.9 & 14525.0 & 0.00 & 0.15 & 1.00 \\
\hline Depth + Salinity + BayDist + Area + HuntLevee + Island + Breached & 12 & 14502.0 & 14526.1 & 1.06 & 0.09 & 1.70 \\
\hline Depth + Salinity + BayDist + UrbanDist + Area + Island + Breached & 12 & 14502.1 & 14526.2 & 1.18 & 0.08 & 1.80 \\
\hline Depth + Salinity + BayDist + Area + OpenLevee + Island + Breached & 12 & 14502.8 & 14526.9 & 1.88 & 0.06 & 2.56 \\
\hline Depth + Salinity + BayDist + Area + Island + Breached + DepthSD & 12 & 14502.8 & 14526.9 & 1.92 & 0.06 & 2.61 \\
\hline \multicolumn{7}{|l|}{ Depth + Salinity + BayDist + UrbanDist + Area + HuntLevee + Island +} \\
\hline Breached & 13 & 14501.2 & 14527.3 & 2.27 & 0.05 & 3.12 \\
\hline Depth + Salinity + BayDist + Area + Island & 10 & 14507.5 & 14527.6 & 2.56 & 0.04 & 3.60 \\
\hline \multicolumn{7}{|l|}{ Depth + Salinity + BayDist + Area + HuntLevee + Island + Breached +} \\
\hline DepthSD & 13 & 14501.8 & 14528.0 & 2.95 & 0.03 & 4.38 \\
\hline \multicolumn{7}{|l|}{ Depth + Salinity + BayDist + Area + OpenLevee + HuntLevee + Island +} \\
\hline Breached & 13 & 14501.9 & 14528.0 & 2.99 & 0.03 & 4.47 \\
\hline \multicolumn{7}{|l|}{ Depth + Salinity + BayDist + UrbanDist + Area + Island + Breached +} \\
\hline \multicolumn{7}{|l|}{ Depth + Salinity + BayDist + UrbanDist + Area + OpenLevee + Island +} \\
\hline Breached & 13 & 14502.0 & 14528.2 & 3.13 & 0.03 & 4.79 \\
\hline Depth + Salinity + BayDist + Area + HuntLevee + Island & 11 & 14506.5 & 14528.6 & 3.58 & 0.03 & 5.99 \\
\hline \multicolumn{7}{|l|}{ Depth + Salinity + BayDist + Area + OpenLevee + Island + Breached +} \\
\hline DepthSD & 13 & 14502.6 & 14528.7 & 3.67 & 0.02 & 6.28 \\
\hline Depth + Salinity + BayDist + UrbanDist + Area + Island & 11 & 14506.8 & 14528.9 & 3.92 & 0.02 & 7.10 \\
\hline $\begin{array}{l}\text { Null Model } \\
\text { Roosting }\end{array}$ & 5 & 14760.6 & 14770.7 & 245.65 & $6.95 \mathrm{E}-55$ & $2.20 \mathrm{E}+53$ \\
\hline \multicolumn{7}{|l|}{ Depth + Salinity + BayDist + UrbanDist + Area + HuntLevee + Island +} \\
\hline Breached & 13 & 24693.4 & 24719.5 & 0.00 & 0.28 & 1.00 \\
\hline \multicolumn{7}{|l|}{ Depth + Salinity + BayDist + UrbanDist + Area + OpenLevee + } \\
\hline HuntLevee + Island + Breached & 14 & 24693.2 & 24721.4 & 1.82 & 0.11 & 2.48 \\
\hline \multicolumn{7}{|l|}{ Depth + Salinity + BayDist + UrbanDist + Area + HuntLevee + Island +} \\
\hline \multicolumn{7}{|l|}{ Depth + Salinity + BayDist + Area + OpenLevee + HuntLevee + Island +} \\
\hline \multicolumn{7}{|l|}{ Depth + Salinity + BayDist + Area + HuntLevee + Island + Breached +} \\
\hline DepthSD & 13 & 24696.2 & 24722.3 & 2.80 & 0.07 & 4.06 \\
\hline \multicolumn{6}{|l|}{ Depth + Salinity + BayDist + UrbanDist + Area + OpenLevee +} & 5.99 \\
\hline HuntLevee + Island + Breached + DepthSD & 15 & 24693.2 & 24723.4 & 3.84 & 0.04 & 6.82 \\
\hline Null Model & 5 & 25173.8 & 25183.8 & 464.29 & 4.17E-102 & $6.61 \mathrm{E}+100$ \\
\hline
\end{tabular}


Table 22. The ranking of candidate models at the pond scale for foraging American avocets in the former salt production ponds of South San Francisco Bay, California.

[Models are ranked by differences in Akaike's information criterion and only models of $\triangle \mathrm{AIC}<4$ and the null model are presented. Birds were surveyed across the former salt ponds during October through April 2002-2015. Variables considered included the mean pond depth (Depth), the presence of islands (Island), variation in topography (DepthSD), \% of pond levee open to hunting (HuntLevee), \% of pond levee open to the public (OpenLevee), pond area (Area), water salinity, pond distance to urban areas (UrbanDist), and pond distance to the bay (BayDist). k: Number of estimated parameters in the model. AICc: Second-order Akaike's information criterion. $\triangle$ AICc: The difference between the AICc of the current model and the AICc of the top model. wi: Akaike weight-likelihood of the model relative to candidate models. Evidence ratio: The weight of evidence that the current model is inferior to the top model]

\begin{tabular}{|c|c|c|c|c|c|c|}
\hline Model name & k & $-2 \log L$ & AlCc & $\Delta \mathrm{AICc}$ & $\mathbf{w}_{\mathrm{i}}$ & $\begin{array}{c}\text { Evidence } \\
\text { ratio }\end{array}$ \\
\hline Depth + BayDist + HuntLevee + Island + DepthSD & 10 & 12182.4 & 12202.5 & 0.00 & 0.04 & 1.00 \\
\hline Depth + BayDist + Island + DepthSD & 9 & 12184.8 & 12202.9 & 0.37 & 0.04 & 1.20 \\
\hline Depth + BayDist + OpenLevee + HuntLevee + Island + DepthSD & 11 & 12181.0 & 12203.1 & 0.58 & 0.03 & 1.33 \\
\hline Depth + BayDist + OpenLevee + Island + DepthSD & 10 & 12183.0 & 12203.1 & 0.60 & 0.03 & 1.35 \\
\hline Depth + HuntLevee + Island + DepthSD & 9 & 12185.1 & 12203.1 & 0.61 & 0.03 & 1.35 \\
\hline Depth + BayDist + Island & 8 & 12187.2 & 12203.25 & 0.73 & 0.03 & 1.44 \\
\hline Depth + Island + DepthSD & 8 & 12187.5 & 12203.6 & 1.05 & 0.03 & 1.69 \\
\hline Depth + OpenLevee + HuntLevee + Island + DepthSD & 10 & 12183.7 & 12203.7 & 1.22 & 0.02 & 1.84 \\
\hline Depth + OpenLevee + Island + DepthSD & 9 & 12185.8 & 12203.8 & 1.33 & 0.02 & 1.94 \\
\hline Depth + BayDist + UrbanDist + HuntLevee + Island + DepthSD & 11 & 12182.1 & 12204.2 & 1.66 & 0.02 & 2.29 \\
\hline Depth + Salinity + BayDist + HuntLevee + Island + DepthSD & 11 & 12182.2 & 12204.3 & 1.82 & 0.02 & 2.48 \\
\hline Depth + BayDist + UrbanDist + Island + DepthSD & 10 & 12184.4 & 12204.5 & 1.98 & 0.02 & 2.69 \\
\hline Depth + BayDist + Area + HuntLevee + Island + DepthSD & 11 & 12182.4 & 12204.5 & 2.02 & 0.02 & 2.74 \\
\hline Depth + UrbanDist + HuntLevee + Island + DepthSD & 10 & 12184.5 & 12204.6 & 2.06 & 0.02 & 2.80 \\
\hline Depth + Salinity + BayDist + Island + DepthSD & 10 & 12184.6 & 12204.7 & 2.20 & 0.01 & 3.00 \\
\hline Depth + BayDist + Area + Island + Breached & 10 & 12184.6 & 12204.7 & 2.20 & 0.01 & 3.00 \\
\hline Depth + BayDist + UrbanDist + OpenLevee + Island + DepthSD & 11 & 12182.7 & 12204.8 & 2.26 & 0.01 & 3.09 \\
\hline \multicolumn{7}{|l|}{ Depth + BayDist + UrbanDist + OpenLevee + HuntLevee + Island +} \\
\hline DepthSD & 12 & 12180.7 & 12204.8 & 2.27 & 0.01 & 3.11 \\
\hline Depth + BayDist + UrbanDist + Island & 9 & 12186.7 & 12204.8 & 2.29 & 0.01 & 3.14 \\
\hline Depth + BayDist + OpenLevee + Island & 9 & 12186.8 & 12204.8 & 2.33 & 0.01 & 3.20 \\
\hline Depth + BayDist + Area + Island + DepthSD & 10 & 12184.8 & 12204.9 & 2.38 & 0.01 & 3.29 \\
\hline Depth + UrbanDist + Island + DepthSD & 9 & 12186.9 & 12205.0 & 2.45 & 0.01 & 3.40 \\
\hline Depth + Salinity + BayDist + OpenLevee + HuntLevee + Island + DepthSD & 12 & 12180.9 & 12205.0 & 2.45 & 0.01 & 3.41 \\
\hline Depth + Salinity + BayDist + Island & 9 & 12186.9 & 12205.0 & 2.49 & 0.01 & 3.47 \\
\hline Depth + Salinity + BayDist + OpenLevee + Island + DepthSD & 11 & 12182.9 & 12205.0 & 2.50 & 0.01 & 3.48 \\
\hline Depth + Salinity + HuntLevee + Island + DepthSD & 10 & 12185.0 & 12205.0 & 2.52 & 0.01 & 3.53 \\
\hline Depth + BayDist + Area + OpenLevee + HuntLevee + Island + DepthSD & 12 & 12181.0 & 12205.1 & 2.55 & 0.01 & 3.58 \\
\hline Depth + BayDist + Area + OpenLevee + Island + DepthSD & 11 & 12183.0 & 12205.1 & 2.58 & 0.01 & 3.62 \\
\hline Depth + Area + HuntLevee + Island + DepthSD & 10 & 12185.1 & 12205.1 & 2.62 & 0.01 & 3.71 \\
\hline Depth + UrbanDist + OpenLevee + HuntLevee + Island + DepthSD & 11 & 12183.1 & 12205.2 & 2.72 & 0.01 & 3.89 \\
\hline Depth + BayDist + Area + Island & 9 & 12187.2 & 12205.2 & 2.73 & 0.01 & 3.91 \\
\hline Depth + UrbanDist + OpenLevee + Island + DepthSD & 10 & 12185.2 & 12205.3 & 2.78 & 0.01 & 4.01 \\
\hline Depth + Salinity + Island + DepthSD & 9 & 12187.4 & 12205.5 & 2.97 & 0.01 & 4.41 \\
\hline Depth + Area + Island + DepthSD & 9 & 12187.5 & 12205.6 & 3.05 & 0.01 & 4.59 \\
\hline Depth + Salinity + OpenLevee + HuntLevee + Island + DepthSD & 11 & 12183.6 & 12205.7 & 3.16 & 0.01 & 4.84 \\
\hline Depth + Area + OpenLevee + HuntLevee + Island + DepthSD & 11 & 12183.6 & 12205.7 & 3.22 & 0.01 & 4.99 \\
\hline Depth + Salinity + OpenLevee + Island + DepthSD & 10 & 12185.7 & 12205.8 & 3.28 & 0.01 & 5.16 \\
\hline
\end{tabular}




\begin{tabular}{|c|c|c|c|c|c|c|}
\hline Model name & k & $-2 \log L$ & AlCc & $\triangle \mathrm{AICc}$ & $\mathbf{w}_{\mathrm{i}}$ & $\begin{array}{c}\begin{array}{c}\text { Evidence } \\
\text { ratio }\end{array} \\
\end{array}$ \\
\hline Depth + Area + OpenLevee + Island + DepthSD & 10 & 12185.8 & 12205.8 & 3.32 & 0.01 & 5.26 \\
\hline Depth + Salinity + BayDist + UrbanDist + HuntLevee + Island + DepthSD & 12 & 12181.8 & 12205.9 & 3.41 & 0.01 & 5.51 \\
\hline Depth + BayDist + UrbanDist + Area + HuntLevee + Island + DepthSD & 12 & 12182.1 & 12206.2 & 3.65 & 0.01 & 6.21 \\
\hline Depth + Salinity + BayDist + UrbanDist + Island + DepthSD & 11 & 12184.2 & 12206.3 & 3.76 & 0.01 & 6.54 \\
\hline Depth + Salinity + BayDist + Area + HuntLevee + Island + DepthSD & 12 & 12182.2 & 12206.4 & 3.83 & 0.01 & 6.79 \\
\hline Depth + HuntLevee + DepthSD & 8 & 12190.3 & 12206.4 & 3.85 & 0.01 & 6.87 \\
\hline Depth + Salinity + UrbanDist + HuntLevee + Island + DepthSD & 11 & 12184.3 & 12206.4 & 3.90 & 0.01 & 7.01 \\
\hline Depth + BayDist + UrbanDist + OpenLevee + Island & 10 & 12186.3 & 12206.4 & 3.90 & 0.01 & 7.03 \\
\hline Depth + Salinity + BayDist + UrbanDist + Island & 10 & 12186.4 & 12206.5 & 3.96 & 0.01 & 7.24 \\
\hline Depth + BayDist + UrbanDist + Area + Island + DepthSD & 11 & 12184.4 & 12206.5 & 3.98 & 0.01 & 7.30 \\
\hline Null Model & 5 & 12224.2 & 12234.2 & 31.68 & $5.75 \mathrm{E}-09$ & 7588217 \\
\hline
\end{tabular}


Table 23. The ranking of candidate models at the pond scale for roosting American avocets in the former salt production ponds of South San Francisco Bay, California.

[Models are ranked by differences in Akaike's information criterion and only models of $\triangle \mathrm{AIC}<4$ and the null model are presented. Birds were surveyed across the former salt ponds during October through April 2002-2015. Variables considered included the mean pond depth (Depth), the presence of islands (Island), variation in topography (DepthSD), \% of pond levee open to hunting (HuntLevee), \% of pond levee open to the public (OpenLevee), pond area (Area), water salinity, pond distance to urban areas (UrbanDist), and pond distance to the bay (BayDist). k: Number of estimated parameters in the model. AICc: Second-order Akaike's information criterion. $\triangle$ AICc: The difference between the AICc of the current model and the AICc of the top model. wi: Akaike weight-likelihood of the model relative to candidate models. Evidence ratio: The weight of evidence that the current model is inferior to the top model]

\begin{tabular}{|c|c|c|c|c|c|c|}
\hline Model name & k & $-2 \log L$ & AICc & $\triangle \mathrm{AICc}$ & $w_{i}$ & $\begin{array}{c}\text { Evidence } \\
\text { ratio }\end{array}$ \\
\hline Depth + Salinity + BayDist + HuntLevee + Island + DepthSD & 11 & 15837.0 & 15859.1 & 0.00 & 0.05 & 1.00 \\
\hline Depth + Salinity + HuntLevee + Island + DepthSD & 10 & 15839.2 & 15859.3 & 0.16 & 0.05 & 1.09 \\
\hline Depth + HuntLevee + Island + DepthSD & 9 & 15841.6 & 15859.6 & 0.53 & 0.04 & 1.30 \\
\hline Depth + BayDist + HuntLevee + Island + DepthSD & 10 & 15839.6 & 15859.6 & 0.54 & 0.04 & 1.31 \\
\hline Depth + Salinity + BayDist + Island + DepthSD & 10 & 15840.4 & 15860.5 & 1.42 & 0.03 & 2.04 \\
\hline Depth + Salinity + Island + DepthSD & 9 & 15842.6 & 15860.7 & 1.61 & 0.02 & 2.24 \\
\hline Depth + Island + DepthSD & 8 & 15844.7 & 15860.8 & 1.68 & 0.02 & 2.31 \\
\hline Depth + BayDist + Island + DepthSD & 9 & 15842.7 & 15860.8 & 1.69 & 0.02 & 2.33 \\
\hline Depth + Salinity + BayDist + UrbanDist + HuntLevee + Island + DepthSD & 12 & 15837.0 & 15861.1 & 2.00 & 0.02 & 2.71 \\
\hline Depth + Salinity + BayDist + Area + HuntLevee + Island + DepthSD & 12 & 15837.0 & 15861.1 & 2.00 & 0.02 & 2.71 \\
\hline Depth + Salinity + BayDist + HuntLevee + Island + Breached + DepthSD & 12 & 15837.0 & 15861.1 & 2.00 & 0.02 & 2.71 \\
\hline \multicolumn{7}{|l|}{ Depth + Salinity + BayDist + OpenLevee + HuntLevee + Island +} \\
\hline DepthSD & 12 & 15837.0 & 15861.1 & 2.02 & 0.02 & 2.74 \\
\hline Depth + Salinity + HuntLevee + Island + Breached + DepthSD & 11 & 15839.4 & 15861.2 & 2.14 & 0.02 & 2.92 \\
\hline Depth + Salinity + UrbanDist + HuntLevee + Island + DepthSD & 11 & 15839.2 & 15861.3 & 2.18 & 0.02 & 2.97 \\
\hline Depth + Salinity + Area + HuntLevee + Island + DepthSD & 11 & 15839.2 & 15861.3 & 2.18 & 0.02 & 2.97 \\
\hline Depth + Salinity + OpenLevee + HuntLevee + Island + DepthSD & 11 & 15839.2 & 15861.3 & 2.18 & 0.02 & 2.97 \\
\hline Depth + HuntLevee + Island + Breached + DepthSD & 10 & 15841.3 & 15861.4 & 2.32 & 0.02 & 3.20 \\
\hline Depth + BayDist + UrbanDist + HuntLevee + Island + DepthSD & 11 & 15839.5 & 15861.6 & 2.50 & 0.02 & 3.49 \\
\hline Depth + BayDist + HuntLevee + Island + Breached + DepthSD & 11 & 15839.5 & 15861.6 & 2.52 & 0.02 & 3.53 \\
\hline Depth + UrbanDist + HuntLevee + Island + DepthSD & 10 & 15841.5 & 15861.6 & 2.52 & 0.02 & 3.53 \\
\hline Depth + BayDist + Area + HuntLevee + Island + DepthSD & 11 & 15839.5 & 15861.6 & 2.54 & 0.02 & 3.56 \\
\hline Depth + Area + HuntLevee + Island + DepthSD & 10 & 15841.5 & 15861.6 & 2.54 & 0.01 & 3.57 \\
\hline Depth + OpenLevee + HuntLevee + Island + DepthSD & 10 & 15841.5 & 15861.6 & 2.54 & 0.01 & 3.57 \\
\hline Depth + BayDist + OpenLevee + HuntLevee + Island + DepthSD & 11 & 15839.5 & 15861.7 & 2.56 & 0.01 & 3.60 \\
\hline Depth + Island + Breached + DepthSD & 9 & 15844.4 & 15862.4 & 3.35 & 0.01 & 5.34 \\
\hline Depth + Salinity + BayDist + UrbanDist + Island + DepthSD & 11 & 15840.4 & 15862.5 & 3.44 & 0.01 & 5.58 \\
\hline Depth + Salinity + BayDist + Area + Island + DepthSD & 11 & 15840.4 & 15862.5 & 3.44 & 0.01 & 5.58 \\
\hline Depth + Salinity + BayDist + OpenLevee + Island + DepthSD & 11 & 15840.4 & 15862.5 & 3.44 & 0.01 & 5.58 \\
\hline Depth + Salinity + BayDist + Island + Breached + DepthSD & 11 & 15840.4 & 15862.5 & 3.44 & 0.01 & 5.58 \\
\hline Depth + BayDist + Island + Breached + DepthSD & 10 & 15842.6 & 15862.7 & 3.58 & 0.01 & 6.00 \\
\hline Depth + Salinity + UrbanDist + Island + DepthSD & 10 & 15842.6 & 15862.7 & 3.62 & 0.01 & 6.12 \\
\hline Depth + Salinity + Area + Island + DepthSD & 10 & 15842.6 & 15862.7 & 3.62 & 0.01 & 6.12 \\
\hline Depth + Salinity + OpenLevee + Island + DepthSD & 10 & 15842.6 & 15862.7 & 3.62 & 0.01 & 6.12 \\
\hline Depth + BayDist + UrbanDist + Island + DepthSD & 10 & 15842.7 & 15862.8 & 3.66 & 0.01 & 6.25 \\
\hline Depth + OpenLevee + Island + DepthSD & 9 & 15844.7 & 15862.8 & 3.67 & 0.01 & 6.27 \\
\hline Depth + BayDist + Area + Island + DepthSD & 10 & 15842.7 & 15862.8 & 3.68 & 0.01 & 6.31 \\
\hline Depth + UrbanDist + Island + DepthSD & 9 & 15844.7 & 15862.8 & 3.69 & 0.01 & 6.33 \\
\hline
\end{tabular}




\begin{tabular}{|c|c|c|c|c|c|c|}
\hline Model name & k & $-2 \log L$ & AICc & $\triangle \mathrm{AICc}$ & $w_{i}$ & $\begin{array}{c}\text { Evidence } \\
\text { ratio }\end{array}$ \\
\hline Depth + Area + Island + DepthSD & 9 & 15844.7 & 15862.8 & 3.69 & 0.01 & 6.33 \\
\hline Depth + BayDist + OpenLevee + Island + DepthSD & 10 & 15842.7 & 15862.8 & 3.70 & 0.01 & 6.37 \\
\hline \multicolumn{7}{|c|}{ Depth + Salinity + BayDist + UrbanDist + Area + HuntLevee + Island +} \\
\hline DepthSD & 13 & 15837.0 & 15863.1 & 3.99 & 0.01 & 7.37 \\
\hline Null Model & 5 & 15919.0 & 15929.0 & 69.93 & $3.49 \mathrm{E}-17$ & $1.53 \mathrm{E}+15$ \\
\hline
\end{tabular}


Table 24. The ranking of candidate models at the pond scale for foraging and roosting eared grebes in the active salt production ponds of South San Francisco Bay, California.

[Models are ranked by differences in Akaike's information criterion and only models of $\triangle \mathrm{AIC}<4$ and the null model are presented. Birds were surveyed across the former salt ponds during October through April 2002-2015. Variables considered included the mean pond depth (Depth), the presence of islands (Island), variation in topography (DepthSD), \% of pond levee open to hunting (HuntLevee), \% of pond levee open to the public (OpenLevee), pond area (Area), water salinity, pond distance to urban areas (UrbanDist), and pond distance to the bay (BayDist). k: Number of estimated parameters in the model. AICc: Second-order Akaike's information criterion. $\triangle$ AICc: The difference between the AICc of the current model and the AICc of the top model. wi: Akaike weight-likelihood of the model relative to candidate models. Evidence ratio: The weight of evidence that the current model is inferior to the top model]

\begin{tabular}{|c|c|c|c|c|c|c|}
\hline Model name & k & $-2 \log L$ & AICc & $\triangle \mathrm{AICc}$ & $w_{i}$ & $\begin{array}{l}\text { Evidence } \\
\text { ratio }\end{array}$ \\
\hline \multicolumn{7}{|l|}{ Foraging } \\
\hline Depth + Salinity + UrbanDist + Area & 9 & 7555.9 & 7574.1 & 0.00 & 0.21 & 1.00 \\
\hline Depth + Salinity + BayDist + Area & 9 & 7556.1 & 7574.3 & 0.16 & 0.19 & 1.08 \\
\hline Depth + Salinity + BayDist + UrbanDist + Area & 10 & 7555.6 & 7575.8 & 1.68 & 0.09 & 2.31 \\
\hline Depth + UrbanDist + Area & 8 & 7559.7 & 7575.8 & 1.71 & 0.09 & 2.35 \\
\hline Depth + BayDist + Area & 8 & 7560.0 & 7576.1 & 2.05 & 0.07 & 2.78 \\
\hline Salinity + UrbanDist + Area & 8 & 7560.5 & 7576.6 & 2.51 & 0.06 & 3.50 \\
\hline Depth + Salinity + Area & 8 & 7561.5 & 7577.6 & 3.51 & 0.04 & 5.78 \\
\hline Depth + BayDist + UrbanDist + Area & 9 & 7559.5 & 7577.7 & 3.60 & 0.03 & 6.05 \\
\hline Depth + Area & 7 & 7563.7 & 7577.8 & 3.70 & 0.03 & 6.36 \\
\hline Depth + Salinity + BayDist & 8 & 7561.9 & 7578.1 & 3.97 & 0.03 & 7.27 \\
\hline Null Model & 5 & 7574.5 & 7584.6 & 10.49 & 0.00 & 189.94 \\
\hline \multicolumn{7}{|l|}{ Roosting } \\
\hline Depth + Salinity + BayDist + Area & 9 & 6838.2 & 6856.4 & 0.00 & 0.43 & 1.00 \\
\hline Depth + Salinity + UrbanDist + Area & 9 & 6838.9 & 6857.1 & 0.72 & 0.30 & 1.43 \\
\hline Depth + Salinity + BayDist + UrbanDist + Area & 10 & 6837.9 & 6858.1 & 1.70 & 0.18 & 2.33 \\
\hline Null Model & 5 & 6900.0 & 6910.1 & 53.69 & $9.35 \mathrm{E}-13$ & $4.56 \mathrm{E}+11$ \\
\hline
\end{tabular}


Table 25. Variable importance values for significant model-averaged coefficients describing foraging and roosting waterbird guild abundance at the pond scale, in restoration ponds in South San Francisco Bay, California.

[Numbers not highlighted indicate a significant positive relation, and numbers highlighted in gray indicate a significant negative relation. Higher values indicate increased importance of the variable for a given guild. Distance to landfill was included only in the models for gulls and is not applicable (N/A) to all other guilds. "--" indicates no significant relation was observed]

\begin{tabular}{|c|c|c|c|c|c|c|c|c|}
\hline Characteristic & $\begin{array}{l}\text { Dabbling } \\
\text { ducks }\end{array}$ & $\begin{array}{l}\text { Diving } \\
\text { ducks }\end{array}$ & $\begin{array}{c}\text { Medium } \\
\text { shorebirds }\end{array}$ & $\begin{array}{c}\text { Small } \\
\text { shorebirds }\end{array}$ & Gulls & Piscivores & Terns & Waders \\
\hline \multicolumn{9}{|c|}{ Foraging } \\
\hline Depth & -- & 1 & 1 & 1 & 0.98 & 1 & -- & 1 \\
\hline Salinity & 1 & 1 & -- & -- & 0.99 & 1 & 1 & 1 \\
\hline Pond area & -- & 0.98 & -- & -- & 0.98 & 1 & 0.96 & 0.96 \\
\hline Distance to San & & & & & & & & \\
\hline Francisco Bay & -- & 0.99 & -- & -- & 0.94 & -- & -- & -- \\
\hline $\begin{array}{l}\text { Distance to urban } \\
\text { areas }\end{array}$ & -- & -- & -- & -- & -- & -- & -- & -- \\
\hline Islands & 1 & 0.95 & -- & 1 & -- & 0.98 & 1 & 1 \\
\hline $\begin{array}{l}\text { Percentage of levees } \\
\text { open to hunting }\end{array}$ & -- & -- & -- & -- & 1 & 0.98 & -- & -- \\
\hline Topography & -- & -- & -- & -- & -- & -- & -- & -- \\
\hline Breached & -- & 1 & -- & 1 & -- & 1 & -- & -- \\
\hline $\begin{array}{l}\text { Percentage of levees } \\
\text { open to public }\end{array}$ & -- & - & -- & 0.93 & -- & - & -- & -- \\
\hline Distance to landfill & $\mathrm{N} / \mathrm{A}$ & N/A & $\mathrm{N} / \mathrm{A}$ & N/A & -- & N/A & $\mathrm{N} / \mathrm{A}$ & N/A \\
\hline \multicolumn{9}{|c|}{ Roosting } \\
\hline Depth & -- & 1 & 1 & 1 & 0.99 & 1 & -- & -- \\
\hline Salinity & 1 & 1 & -- & -- & -- & 1 & 1 & 1 \\
\hline $\begin{array}{l}\text { Pond area } \\
\text { Distance to San }\end{array}$ & -- & 1 & -- & -- & 0.99 & 1 & 0.98 & 0.99 \\
\hline Francisco Bay & -- & 1 & -- & -- & 0.95 & -- & -- & -- \\
\hline $\begin{array}{l}\text { Distance to urban } \\
\text { areas }\end{array}$ & -- & -- & -- & -- & -- & -- & -- & -- \\
\hline Islands & 1 & 1 & 1 & 0.94 & 1 & 0.93 & 1 & 1 \\
\hline $\begin{array}{l}\text { Percentage of levees } \\
\text { open to hunting }\end{array}$ & 1 & -- & -- & -- & -- & -- & 1 & 0.99 \\
\hline Topography & 0.97 & -- & 0.94 & -- & -- & -- & -- & -- \\
\hline Breached & -- & 1 & -- & 0.93 & -- & 1 & -- & -- \\
\hline $\begin{array}{l}\text { Percentage of levees } \\
\text { open to public }\end{array}$ & -- & -- & -- & -- & 0.93 & -- & -- & -- \\
\hline Distance to landfill & N/A & N/A & N/A & N/A & 0.98 & N/A & N/A & N/A \\
\hline
\end{tabular}


Table 26. Optimal habitat characteristic values for waterbird guilds at the pond scale, as determined from General Additive Models (GAMs), in restoration ponds, South San Francisco Bay, California.

[Variables with a linear trend (islands and breached), are not included here. "---" indicates no significant relation was observed. Abbreviations and symbols: $\mathrm{km}$, kilometer; $\mathrm{km}^{2}$, square kilometer; $\mathrm{m}$, meter, ppt, parts per thousand; >, greater than; $\geq$, greater than or equal to; $<$, less than; \%, percent]

\begin{tabular}{|c|c|c|c|c|c|c|c|c|}
\hline & $\begin{array}{l}\text { Dabbling } \\
\text { ducks }\end{array}$ & $\begin{array}{l}\text { Diving } \\
\text { ducks }\end{array}$ & $\begin{array}{c}\text { Medium } \\
\text { shorebirds }\end{array}$ & $\begin{array}{c}\text { Small } \\
\text { shorebirds }\end{array}$ & Gulls & Piscivores & Terns & Waders \\
\hline \multicolumn{9}{|c|}{ Foraging } \\
\hline Depth (m) & -- & 0.75 & 0.13 & 0 & 1.48 & $3 \geq 0.4$ & -- & 0.4 \\
\hline Salinity (ppt) & 6 & $<17$ & -- & -- & 124 & 4 & 15 & 1 \\
\hline Pond area $\left(\mathrm{km}^{2}\right)$ & -- & 1.25 & -- & -- & $>0$ & $>0$ & $>0.75$ & $>0$ \\
\hline $\begin{array}{l}\text { Distance to San Francisco } \\
\text { Bay }(\mathrm{km})\end{array}$ & -- & 1.1 & -- & -- & $>0.9$ & -- & -- & -- \\
\hline $\begin{array}{l}\text { Distance to urban areas } \\
(\mathrm{km})\end{array}$ & -- & -- & -- & -- & -- & -- & -- & -- \\
\hline $\begin{array}{l}\text { Levees open to hunting } \\
(\%)\end{array}$ & -- & -- & -- & -- & $>78$ & 25 & -- & -- \\
\hline Topography (m) & -- & -- & -- & -- & -- & -- & -- & -- \\
\hline Levees open to public (\%) & -- & -- & -- & 0 and 70 & -- & -- & -- & -- \\
\hline Distance to landfill $(\mathrm{km})$ & -- & -- & -- & -- & -- & -- & -- & -- \\
\hline \multicolumn{9}{|c|}{ Roosting } \\
\hline Depth (m) & -- & 1.5 & 0.25 & 0 & 0.3 & $>1.25$ & -- & -- \\
\hline Salinity (ppt) & 6 & $<5$ & -- & -- & -- & & 15 & 17 \\
\hline Pond area $\left(\mathrm{km}^{2}\right)$ & -- & 1.25 & -- & -- & $>1.75$ & $5>0.5$ & $>1.5$ & $>0$ \\
\hline $\begin{array}{l}\text { Distance to San Francisco } \\
\text { Bay }(\mathrm{km})\end{array}$ & -- & 1.1 & -- & -- & $>0$ & -- & -- & -- \\
\hline $\begin{array}{l}\text { Distance to urban } \\
\text { areas }(\mathrm{km})\end{array}$ & -- & -- & -- & -- & -- & -- & -- & -- \\
\hline $\begin{array}{l}\text { Levees open to hunting } \\
(\%)\end{array}$ & $<77$ & -- & -- & -- & -- & -- & 100 & 77 \\
\hline Topography (m) & 0.61 & -- & $>0.15$ & -- & -- & -- & -- & -- \\
\hline Levees open to public (\%) & -- & -- & -- & -- & 46 & -- & -- & -- \\
\hline Distance to landfill $(\mathrm{km})$ & -- & -- & -- & -- & 2.8 & -- & -- & -- \\
\hline
\end{tabular}


Table 27. Variable importance values for significant model-averaged coefficients describing foraging and roosting waterbird abundance at the pond scale in restoration ponds, South San Francisco Bay, California.

[Numbers not highlighted indicate a significant positive relation, and numbers highlighted in gray indicate a significant negative relation. Higher values indicate increased importance of the variable for a given species. "--" indicates no significant relation was observed. Abbreviations: American avocet, AMAV; eared grebe, EAGR; northern shoveler, NSHO; ruddy duck, RUDU.

\begin{tabular}{|c|c|c|c|c|}
\hline Characteristic & AMAV & EAGR & NSHO & RUDU \\
\hline \multicolumn{5}{|c|}{ Foraging } \\
\hline Depth & 1 & 1 & -- & 1 \\
\hline Salinity & -- & 1 & 1 & 1 \\
\hline Pond area & -- & 1 & -- & 0.97 \\
\hline Distance to San & & & & \\
\hline Francisco Bay & -- & -- & -- & 0.96 \\
\hline Distance to urban areas & -- & -- & -- & -- \\
\hline Islands & -- & -- & 1 & 1 \\
\hline $\begin{array}{l}\text { Percentage of levees } \\
\text { open to hunting }\end{array}$ & -- & -- & -- & -- \\
\hline Topography & -- & -- & -- & -- \\
\hline Breached & -- & 1 & -- & -- \\
\hline $\begin{array}{l}\text { Percentage of levees } \\
\text { open to public }\end{array}$ & -- & -- & -- & -- \\
\hline \multicolumn{5}{|c|}{ Roosting } \\
\hline Depth & 1 & 1 & -- & 1 \\
\hline Salinity & -- & -- & 1 & 1 \\
\hline Pond area & -- & 1 & -- & 0.99 \\
\hline $\begin{array}{l}\text { Distance to San } \\
\text { Francisco Bay }\end{array}$ & -- & -- & -- & 0.96 \\
\hline Distance to urban areas & -- & -- & -- & -- \\
\hline Islands & 1 & -- & 1 & 1 \\
\hline $\begin{array}{l}\text { Percentage of levees } \\
\text { open to hunting }\end{array}$ & -- & -- & 0.96 & -- \\
\hline Topography & 0.99 & -- & 0.95 & -- \\
\hline Breached & -- & -- & -- & 1 \\
\hline $\begin{array}{l}\text { Percentage of levees } \\
\text { open to public }\end{array}$ & -- & - & -- & -- \\
\hline
\end{tabular}


Table 28. Optimal habitat characteristic values for waterbird species at the pond scale, as determined from General Additive Models (GAMs), in restoration ponds, South San Francisco Bay, California.

[Variables with a linear trend (islands and breached) are not included here. “--” indicates no significant relation was observed. Abbreviations and symbols: American avocet, AMAV; eared grebe, EAGR; northern shoveler, NSHO; ruddy duck, RUDU; km, kilometer; $\mathrm{km}^{2}$, square kilometer; $\mathrm{m}$, meter, ppt, parts per thousand; >, greater than; \%, percent]

\begin{tabular}{lcccc}
\hline & AMAV & EAGR & NSHO & RUDU \\
\hline & Foraging & & & \\
\hline Depth (m) & 0.30 & $>0$ & -- & 0.74 \\
Salinity (ppt) & - & 109 & 8 & 11 \\
Pond area (km²) & -- & 2.30 & -- & 2.35 \\
Distance to San Francisco & & & & \\
$\quad$ Bay (km) & -- & -- & -- & 1.01 \\
Distance to urban areas (km) & - & -- & -- & -- \\
Levees open to hunting (\%) & -- & -- & -- & -- \\
Topography (m) & 0.25 & -- & -- & -- \\
Levees open to public (\%) & -- & -- & -- & -- \\
\hline & Roosting & & & \\
\hline Depth (m) & 0.32 & 1.29 & -- & 1.73 \\
Salinity (ppt) & -- & -- & 6 & 7 \\
Pond area (km ${ }^{2}$ ) & -- & 2.30 & -- & $\geq 1.25$ \\
Distance to San Francisco & & & & \\
$\quad$ Bay (km) & -- & -- & -- & 1.62 \\
Distance to urban areas (km) & -- & -- & -- & -- \\
Levees open to hunting (\%) & -- & -- & 0 and & \\
Topography (m) & 0.25 & -- & 0.61 & -- \\
Levees open to public (\%) & -- & -- & -- & -- \\
\hline
\end{tabular}


Table 29. The ranking of candidate models at the grid scale for foraging and roosting dabbling ducks in the former salt production ponds of South San Francisco Bay, California.

[Models are ranked by differences in Akaike's information criterion and only models of $\triangle \mathrm{AIC}<4$ and the null model are presented. Birds were surveyed across the former salt ponds during October through April 2002-2015. Variables considered included the mean grid depth (Depth), the presence of islands (Island), variation in topography (DepthSD), grid distance to the neared pond levee (LeveeDist), and grid distance to the nearest creak or slough (CreekDist). Grid area was included in every model. k: Number of estimated parameters in the model. AICc: Second-order Akaike's information criterion. $\triangle$ AICc: The difference between the AICc of the current model and the AICc of the top model. $\mathbf{w}_{\mathbf{i}}$ : Akaike weight-likelihood of the model relative to candidate models. Evidence ratio: The weight of evidence that the current model is inferior to the top model]

\begin{tabular}{|c|c|c|c|c|c|c|}
\hline Model name & k & $-2 \log L$ & AICc & $\triangle \mathrm{AICc}$ & $\mathbf{w i}_{\mathrm{i}}$ & $\begin{array}{c}\text { Evidence } \\
\text { ratio }\end{array}$ \\
\hline \multicolumn{7}{|l|}{ Foraging } \\
\hline GridArea + LeveeDist + CreekDist + ElevSD + Island + Depth & 12 & 194715.6 & 194739.6 & 0.00 & 0.40 & 1.00 \\
\hline GridArea + LeveeDist + CreekDist + ElevSD + Depth & 11 & 194719.2 & 194741.2 & 1.60 & 0.18 & 2.22 \\
\hline GridArea + LeveeDist + ElevSD + Island + Depth & 11 & 194720.2 & 194742.2 & 2.60 & 0.11 & 3.67 \\
\hline GridArea + CreekDist + ElevSD + Island + Depth & 11 & 194720.6 & 194742.6 & 3.00 & 0.09 & 4.48 \\
\hline GridArea + LeveeDist + ElevSD + Depth & 10 & 194723.4 & 194743.4 & 3.80 & 0.06 & 6.68 \\
\hline GridArea (null model) & 7 & 194788.0 & 194802.0 & 62.40 & $1.14 \mathrm{E}-14$ & $3.54 \mathrm{E}+13$ \\
\hline \multicolumn{7}{|l|}{ Roosting } \\
\hline GridArea + Island + Depth & 9 & 235178.0 & 235196.0 & 0.00 & 0.24 & 1.00 \\
\hline GridArea + ElevSD + Island + Depth & 10 & 235176.0 & 235196.0 & 0.00 & 0.24 & 1.00 \\
\hline GridArea + LeveeDist + Island + Depth & 10 & 235178.0 & 235198.0 & 2.00 & 0.09 & 2.72 \\
\hline GridArea + CreekDist + Island + Depth & 10 & 235178.0 & 235198.0 & 2.00 & 0.09 & 2.72 \\
\hline GridArea + LeveeDist + CreekDist + Island + Depth & 11 & 235176.0 & 235198.0 & 2.00 & 0.09 & 2.72 \\
\hline GridArea + LeveeDist + ElevSD + Island + Depth & 11 & 235176.0 & 235198.0 & 2.00 & 0.09 & 2.72 \\
\hline GridArea + CreekDist + ElevSD + Island + Depth & 11 & 235176.0 & 235198.0 & 2.00 & 0.09 & 2.72 \\
\hline GridArea (null model) & 7 & 235246.0 & 235260.0 & 64.00 & $3.04 \mathrm{E}-15$ & $7.89 \mathrm{E}+13$ \\
\hline
\end{tabular}


Table 30. The ranking of candidate models at the grid scale for foraging and roosting diving ducks in the former salt production ponds of South San Francisco Bay, California.

[Models are ranked by differences in Akaike's information criterion and only models of $\triangle \mathrm{AIC}<4$ and the null model are presented. Birds were surveyed across the former salt ponds during October through April 2002-2015. Variables considered included the mean grid depth (Depth), the presence of islands (Island), variation in topography (DepthSD), grid distance to the neared pond levee (LeveeDist), and grid distance to the nearest creak or slough (CreekDist). Grid area was included in every model. k: Number of estimated parameters in the model. AICc: Second-order Akaike's information criterion. $\triangle$ AICc: The difference between the AICc of the current model and the AICc of the top model. $\mathbf{w}_{\mathbf{i}}$ : Akaike weight-likelihood of the model relative to candidate models. Evidence ratio: The weight of evidence that the current model is inferior to the top model]

\begin{tabular}{|c|c|c|c|c|c|c|}
\hline Model name & k & $-2 \log L$ & AICc & $\triangle \mathrm{AICC}$ & $w_{i}$ & $\begin{array}{c}\text { Evidence } \\
\text { ratio }\end{array}$ \\
\hline \multicolumn{7}{|l|}{ Foraging } \\
\hline GridArea + LeveeDist + ElevSD + Depth & 10 & 179753.8 & 179773.8 & 0.00 & 0.36 & 1.00 \\
\hline GridArea + LeveeDist + ElevSD + Island + Depth & 11 & 179752.6 & 179774.6 & 0.80 & 0.24 & 1.49 \\
\hline GridArea + LeveeDist + CreekDist + ElevSD + Depth & 11 & 179753.6 & 179775.6 & 1.80 & 0.15 & 2.46 \\
\hline GridArea + LeveeDist + CreekDist + ElevSD + Island + Depth & 12 & 179752.4 & 179776.4 & 2.60 & 0.10 & 3.67 \\
\hline GridArea + ElevSD + Depth & 9 & 179759.8 & 179777.8 & 4.00 & 0.05 & 7.39 \\
\hline GridArea (null model) & 7 & 180175.2 & 180189.2 & 415.40 & $2.26 \mathrm{E}-91$ & $1.59 \mathrm{E}+90$ \\
\hline \multicolumn{7}{|l|}{ Roosting } \\
\hline GridArea + ElevSD + Depth & 9 & 318776.0 & 318794.0 & 0.00 & 0.22 & 1.00 \\
\hline GridArea + Depth & 8 & 318780.0 & 318796.0 & 2.00 & 0.08 & 2.72 \\
\hline GridArea + LeveeDist + Depth & 9 & 318778.0 & 318796.0 & 2.00 & 0.08 & 2.72 \\
\hline GridArea + CreekDist + Depth & 9 & 318778.0 & 318796.0 & 2.00 & 0.08 & 2.72 \\
\hline GridArea + LeveeDist + ElevSD + Depth & 10 & 318776.0 & 318796.0 & 2.00 & 0.08 & 2.72 \\
\hline GridArea + CreekDist + ElevSD + Depth & 10 & 318776.0 & 318796.0 & 2.00 & 0.08 & 2.72 \\
\hline GridArea + ElevSD + Island + Depth & 10 & 318776.0 & 318796.0 & 2.00 & 0.08 & 2.72 \\
\hline GridArea + LeveeDist + CreekDist + ElevSD + Depth & 11 & 318774.0 & 318796.0 & 2.00 & 0.08 & 2.72 \\
\hline GridArea (null model) & 7 & 319796.0 & 319810.0 & 1016.00 & $5.23 \mathrm{E}-222$ & $4.18 \mathrm{E}+220$ \\
\hline
\end{tabular}


Table 31. The ranking of candidate models at the grid scale for foraging and roosting small shorebirds in the former salt production ponds of South San Francisco Bay, California.

[Models are ranked by differences in Akaike's information criterion and only models of $\triangle \mathrm{AIC}<4$ and the null model are presented. Birds were surveyed across the former salt ponds during October through April 2002-2015. Variables considered included the mean grid depth (Depth), the presence of islands (Island), variation in topography (DepthSD), grid distance to the neared pond levee (LeveeDist), and grid distance to the nearest creak or slough (CreekDist). Grid area was included in every model. k: Number of estimated parameters in the model. AICc: Second-order Akaike's information criterion. $\triangle$ AICc: The difference between the AICc of the current model and the AICc of the top model. $\mathbf{w}_{\mathbf{i}}$ : Akaike weight-likelihood of the model relative to candidate models. Evidence ratio: The weight of evidence that the current model is inferior to the top model]

\begin{tabular}{|c|c|c|c|c|c|c|}
\hline Model name & k & $-2 \log L$ & AICc & $\triangle \mathrm{AICc}$ & $\mathbf{w}_{\mathrm{i}}$ & $\begin{array}{c}\text { Evidence } \\
\text { ratio }\end{array}$ \\
\hline \multicolumn{7}{|l|}{ Foraging } \\
\hline GridArea + LeveeDist + CreekDist + ElevSD + Island + Depth & 12 & 168372.0 & 168396.0 & 0.00 & 0.66 & 1.00 \\
\hline GridArea + LeveeDist + CreekDist + ElevSD + Depth & 11 & 168375.8 & 168397.8 & 1.80 & 0.27 & 2.46 \\
\hline GridArea (null model) & 7 & 169836.8 & 169850.8 & 1454.80 & $8.17 \mathrm{e}-317$ & Inf \\
\hline \multicolumn{7}{|l|}{ Roosting ${ }^{g}$} \\
\hline GridArea + LeveeDist + ElevSD + Island + Depth & 11 & 125281.0 & 125303.0 & 0.00 & 1.00 & 1.00 \\
\hline
\end{tabular}
Table 32. The ranking of candidate models at the grid scale for foraging and roosting medium shorebirds in the
former salt production ponds of South San Francisco Bay, California.

[Models are ranked by differences in Akaike's information criterion and only models of $\triangle \mathrm{AIC}<4$ and the null model are presented. Birds were surveyed across the former salt ponds during October through April 2002-2015. Variables considered included the mean grid depth (Depth), the presence of islands (Island), variation in topography (DepthSD), grid distance to the neared pond levee (LeveeDist), and grid distance to the nearest creak or slough (CreekDist). Grid area was included in every model. k: Number of estimated parameters in the model. AICc: Second-order Akaike's information criterion. $\triangle$ AICc: The difference between the AICc of the current model and the AICc of the top model. $\mathbf{w}_{\mathbf{i}}$ : Akaike weight-likelihood of the model relative to candidate models. Evidence ratio: The weight of evidence that the current model is inferior to the top model]

\begin{tabular}{lrrrrrr}
\hline \multicolumn{1}{c}{ Model name } & k & -2LogL & AlCc & AAICc & Wi & $\begin{array}{r}\text { Evidence } \\
\text { ratio }\end{array}$ \\
\hline Foraging & & & & & & \\
GridArea + LeveeDist + CreekDist + ElevSD + Island + Depth & 12 & 105309.0 & 105333.0 & 0.00 & 0.71 & 1.00 \\
GridArea + LeveeDist + ElevSD + Island + Depth & 11 & 105312.8 & 105334.8 & 1.80 & 0.29 & 2.46 \\
GridArea (null model) & 7 & 106183.4 & 106197.4 & 864.40 & $1.41 \mathrm{E}-188$ & $5.03 \mathrm{E}+187$ \\
$\quad$ Roosting & & & & & & \\
GridArea + LeveeDist + CreekDist + ElevSD + Island + Depth & 12 & 117043.4 & 117067.4 & 0.00 & 0.95 & 1.00 \\
GridArea (null model) & 7 & 117591.2 & 117605.2 & 537.80 & $1.58 \mathrm{E}-117$ & $6.04 \mathrm{E}+116$ \\
\hline
\end{tabular}


Table 33. The ranking of candidate models at the grid scale for foraging and roosting waders in the former salt production ponds of South San Francisco Bay, California.

[Models are ranked by differences in Akaike's information criterion and only models of $\triangle \mathrm{AIC}<4$ and the null model are presented. Birds were surveyed across the former salt ponds during October through April 2002-2015. Variables considered included the mean grid depth (Depth), the presence of islands (Island), variation in topography (DepthSD), grid distance to the neared pond levee (LeveeDist), and grid distance to the nearest creak or slough (CreekDist). Grid area was included in every model. k: Number of estimated parameters in the model. AICc: Second-order Akaike's information criterion. $\triangle$ AICc: The difference between the AICc of the current model and the AICc of the top model. $\mathbf{w}_{\mathbf{i}}$ : Akaike weight-likelihood of the model relative to candidate models. Evidence ratio: The weight of evidence that the current model is inferior to the top model]

\begin{tabular}{lrrrrrr}
\hline \multicolumn{1}{c}{ Model name } & k & -2LogL & AlCc & $\Delta$ AICc & W $_{\text {i }}$ & Evidence ratio \\
\hline Foraging & & & & & & \\
GridArea + LeveeDist + ElevSD + Island + Depth & 11 & 54034.8 & 54056.8 & 0.00 & 0.50 & 1.00 \\
GridArea + LeveeDist + CreekDist + ElevSD + Island + Depth & 12 & 54032.8 & 54056.8 & 0.00 & 0.50 & 1.00 \\
GridArea (null model) & 7 & 54420.0 & 54434.0 & 377.20 & $6.16 \mathrm{E}-83$ & $8.08 \mathrm{E}+81$ \\
$\quad$ Roosting & & & & & & \\
GridArea + LeveeDist + ElevSD + Island + Depth & 11 & 49898.0 & 49920.0 & 0.00 & 0.48 & 1.00 \\
GridArea + LeveeDist + CreekDist + ElevSD + Island + Depth & 12 & 49896.0 & 49920.0 & 0.00 & 0.48 & 1.00 \\
GridArea (null model) & 7 & 50192.4 & 50206.4 & 286.40 & $3.11 \mathrm{E}-63$ & $1.55 \mathrm{E}+62$ \\
\hline
\end{tabular}

\section{Table 34. The ranking of candidate models at the grid scale for foraging and roosting piscivores in the former salt} production ponds of South San Francisco Bay, California.

[Models are ranked by differences in Akaike's information criterion and only models of $\triangle \mathrm{AIC}<4$ and the null model are presented. Birds were surveyed across the former salt ponds during October through April 2002-2015. Variables considered included the mean grid depth (Depth), the presence of islands (Island), variation in topography (DepthSD), grid distance to the neared pond levee (LeveeDist), and grid distance to the nearest creak or slough (CreekDist). Grid area was included in every model. k: Number of estimated parameters in the model. AICc: Second-order Akaike's information criterion. $\triangle$ AICc: The difference between the AICc of the current model and the AICc of the top model. wi: Akaike weight-likelihood of the model relative to candidate models. Evidence ratio: The weight of evidence that the current model is inferior to the top model]

\begin{tabular}{lrrrrrr}
\hline \multicolumn{1}{c}{ Model name } & k & -2LogL & AICc & $\Delta$ AICc & Wi & $\begin{array}{r}\text { Evidence } \\
\text { ratio }\end{array}$ \\
\hline Foraging & & & & & & \\
GridArea + LeveeDist + ElevSD + Island + Depth & 11 & 87810.4 & 87832.4 & 0.00 & 0.21 & 1.00 \\
GridArea + LeveeDist + ElevSD + Depth & 10 & 87812.6 & 87832.6 & 0.20 & 0.19 & 1.10 \\
GridArea (null model) & 7 & 87928.2 & 87942.2 & 109.80 & $2.99 \mathrm{E}-25$ & $6.96 \mathrm{E}+23$ \\
$\quad$ Roosting & & & & & & \\
GridArea + LeveeDist + CreekDist + Island + Depth & 11 & 102824.6 & 102846.6 & 0.00 & 0.55 & 1.00 \\
GridArea + LeveeDist + CreekDist + ElevSD + Island + Depth & 12 & 102823.6 & 102847.6 & 1.00 & 0.33 & 1.65 \\
GridArea (null model) & 7 & 102973.6 & 102987.6 & 141.00 & $1.33 \mathrm{E}-31$ & $4.14 \mathrm{E}+30$ \\
\hline
\end{tabular}


Table 35. The ranking of candidate models at the grid scale for foraging and roosting terns in the former salt production ponds of South San Francisco Bay, California.

[Models are ranked by differences in Akaike's information criterion and only models of $\triangle \mathrm{AIC}<4$ and the null model are presented. Birds were surveyed across the former salt ponds during October through April 2002-2015. Variables considered included the mean grid depth (Depth), the presence of islands (Island), variation in topography (DepthSD), grid distance to the neared pond levee (LeveeDist), and grid distance to the nearest creak or slough (CreekDist). Grid area was included in every model. k: Number of estimated parameters in the model. AICc: Second-order Akaike's information criterion. $\triangle$ AICc: The difference between the AICc of the current model and the AICc of the top model. $\mathbf{w}_{\mathbf{i}}$ : Akaike weight-likelihood of the model relative to candidate models. Evidence ratio: The weight of evidence that the current model is inferior to the top model]

\begin{tabular}{lrrrrrr}
\hline \multicolumn{1}{c}{ Model name } & k & -2LogL & AlCc & A AICc & wi & $\begin{array}{c}\text { Evidence } \\
\text { ratio }\end{array}$ \\
\hline Foraging & & & & & & \\
GridArea + LeveeDist + CreekDist + Depth & 10 & 18129.7 & 18149.7 & 0.00 & 0.34 & 1.00 \\
GridArea + LeveeDist + CreekDist + ElevSD + Depth & 11 & 18128.9 & 18150.9 & 1.28 & 0.18 & 1.90 \\
GridArea + LeveeDist + CreekDist + Island + Depth & 11 & 18129.0 & 18151.0 & 1.34 & 0.18 & 1.95 \\
GridArea + LeveeDist + CreekDist + ElevSD + Island + Depth & 12 & 18128.3 & 18152.3 & 2.68 & 0.09 & 3.82 \\
GridArea + LeveeDist + Depth & 9 & 18135.1 & 18153.1 & 3.44 & 0.06 & 5.58 \\
GridArea (Null Model) & 7 & 18159.6 & 18173.6 & 23.92 & $2.20 \mathrm{E}-06$ & $1.56 \mathrm{E}+05$ \\
$\quad$ Roosting & & & & & & \\
GridArea + LeveeDist + ElevSD + Island + Depth & 11 & 24905.4 & 24927.4 & 0.00 & 0.51 & 1.00 \\
GridArea + LeveeDist + Island + Depth & 10 & 24909.2 & 24929.2 & 1.80 & 0.21 & 2.46 \\
GridArea + LeveeDist + CreekDist + ElevSD + Island + Depth & 12 & 24905.2 & 24929.2 & 1.80 & 0.21 & 2.46 \\
GridArea + LeveeDist + CreekDist + Island + Depth & 11 & 24909.2 & 24931.2 & 3.80 & 0.08 & 6.69 \\
GridArea (Null Model) & 7 & 24960.4 & 24974.4 & 47.00 & $3.16 \mathrm{E}-11$ & $1.61 \mathrm{E}+10$ \\
\hline
\end{tabular}

Table 36. The ranking of candidate models at the grid scale for foraging and roosting gulls in the former salt production ponds of South San Francisco Bay, California.

[Models are ranked by differences in Akaike's information criterion and only models of $\triangle \mathrm{AIC}<4$ and the null model are presented. Birds were surveyed across the former salt ponds during October through April 2002-2015. Variables considered included the mean grid depth (Depth), the presence of islands (Island), variation in topography (DepthSD), grid distance to the neared pond levee (LeveeDist), and grid distance to the nearest creak or slough (CreekDist). Grid area was included in every model. k: Number of estimated parameters in the model. AICc: Second-order Akaike's information criterion. $\triangle$ AICc: The difference between the AICc of the current model and the AICc of the top model. $\mathbf{w}_{\mathbf{i}}$ : Akaike weight-likelihood of the model relative to candidate models. Evidence ratio: The weight of evidence that the current model is inferior to the top model]

\begin{tabular}{|c|c|c|c|c|c|c|}
\hline Model name & k & $-2 \log L$ & AICc & $\triangle \mathrm{AICc}$ & $\mathbf{w i}_{\mathrm{i}}$ & $\begin{array}{c}\text { Evidence } \\
\text { ratio }\end{array}$ \\
\hline \multicolumn{7}{|l|}{ Foraging } \\
\hline GridArea + LeveeDist + ElevSD + Island + Depth & 11 & 42409.4 & 42431.4 & 0.00 & 0.40 & 1.00 \\
\hline GridArea + LeveeDist + Island + Depth & 10 & 42412.6 & 42432.6 & 1.20 & 0.22 & 1.82 \\
\hline GridArea + LeveeDist + CreekDist + ElevSD + Island + Depth & 12 & 42409.4 & 42433.4 & 2.00 & 0.15 & 2.72 \\
\hline GridArea + LeveeDist + CreekDist + Island + Depth & 11 & 42412.6 & 42434.6 & 3.20 & 0.08 & 4.95 \\
\hline GridArea + LeveeDist + ElevSD + Depth & 10 & 42414.8 & 42434.8 & 3.40 & 0.07 & 5.47 \\
\hline GridArea (null model) & 7 & 42456.6 & 42470.6 & 39.20 & $1.23 \mathrm{E}-09$ & $3.25 \mathrm{E}+08$ \\
\hline \multicolumn{7}{|l|}{ Roosting } \\
\hline GridArea + LeveeDist + CreekDist + ElevSD + Island + Depth & 12 & 145624.4 & 145648.4 & 0.00 & 0.54 & 1.00 \\
\hline GridArea + LeveeDist + ElevSD + Island + Depth & 11 & 145626.8 & 145648.8 & 0.40 & 0.44 & 1.22 \\
\hline GridArea (null model) & 7 & 145738 & 145752.0 & 103.60 & $1.73 \mathrm{E}-23$ & $3.13 \mathrm{E}+22$ \\
\hline
\end{tabular}


Table 37. The ranking of candidate models at the grid scale for foraging and roosting eared grebes in the former salt production ponds of South San Francisco Bay, California.

[Models are ranked by differences in Akaike's information criterion and only models of $\triangle \mathrm{AIC}<4$ and the null model are presented. Birds were surveyed across the former salt ponds during October through April 2002-2015. Variables considered included the mean grid depth (Depth), the presence of islands (Island), variation in topography (DepthSD), grid distance to the neared pond levee (LeveeDist), and grid distance to the nearest creak or slough (CreekDist). Grid area was included in every model. k: Number of estimated parameters in the model. AICc: Second-order Akaike's information criterion. $\triangle$ AICc: The difference between the AICc of the current model and the AICc of the top model. $\mathbf{w}_{\mathbf{i}}$ : Akaike weight-likelihood of the model relative to candidate models. Evidence ratio: The weight of evidence that the current model is inferior to the top model]

\begin{tabular}{|c|c|c|c|c|c|c|}
\hline Model name & k & $-2 \log L$ & AICc & $\triangle \mathrm{AICC}$ & $\mathrm{w}_{\mathrm{i}}$ & $\begin{array}{c}\text { Evidence } \\
\text { ratio }\end{array}$ \\
\hline \multicolumn{7}{|l|}{ Foraging } \\
\hline GridArea + LeveeDist + ElevSD + Island + Depth & 11 & 88864.2 & 88886.2 & 0.00 & 0.36 & 1.00 \\
\hline GridArea + LeveeDist + Island + Depth & 10 & 88867.0 & 88887.0 & 0.80 & 0.24 & 1.49 \\
\hline GridArea + LeveeDist + CreekDist + ElevSD + Island + Depth & 12 & 88863.8 & 88887.8 & 1.60 & 0.16 & 2.23 \\
\hline GridArea + LeveeDist + CreekDist + Island + Depth & 11 & 88866.2 & 88888.2 & 2.00 & 0.13 & 2.72 \\
\hline GridArea (null model) & 7 & 89207.8 & 89221.8 & 335.60 & 4.84E-74 & $7.49 \mathrm{E}+72$ \\
\hline \multicolumn{7}{|l|}{ Roosting } \\
\hline GridArea + LeveeDist + CreekDist + ElevSD + Island + Depth & 12 & 60658.6 & 60682.6 & 0.00 & 0.38 & 1.00 \\
\hline GridArea + LeveeDist + CreekDist + Island + Depth & 11 & 60661.2 & 60683.2 & 0.60 & 0.28 & 1.35 \\
\hline GridArea + LeveeDist + ElevSD + Island + Depth & 11 & 60661.6 & 60683.6 & 1.00 & 0.23 & 1.65 \\
\hline GridArea + LeveeDist + Island + Depth & 10 & 60665.4 & 60685.4 & 2.80 & 0.09 & 4.05 \\
\hline GridArea (null model) & 7 & 61031.8 & 61045.8 & 363.20 & $5.20 \mathrm{E}-80$ & $7.37 \mathrm{E}+78$ \\
\hline
\end{tabular}

Table 38. The ranking of candidate models at the grid scale for foraging and roosting northern shovelers in the former salt production ponds of South San Francisco Bay, California.

[Models are ranked by differences in Akaike's information criterion and only models of $\triangle \mathrm{AIC}<4$ and the null model are presented. Birds were surveyed across the former salt ponds during October through April 2002-2015. Variables considered included the mean grid depth (Depth), the presence of islands (Island), variation in topography (DepthSD), grid distance to the neared pond levee (LeveeDist), and grid distance to the nearest creak or slough (CreekDist). Grid area was included in every model. k: Number of estimated parameters in the model. AICc: Second-order Akaike's information criterion. $\triangle$ AICc: The difference between the AICc of the current model and the AICc of the top model. wi: Akaike weight-likelihood of the model relative to candidate models. Evidence ratio: The weight of evidence that the current model is inferior to the top model]

\begin{tabular}{lrrrrrr}
\hline \multicolumn{1}{c}{ Model name } & k & -2LogL & AlCc & $\Delta$ AlCc & wi & $\begin{array}{r}\text { Evidence } \\
\text { ratio }\end{array}$ \\
\hline Foraging & & & & & & \\
GridArea + LeveeDist + ElevSD + Island + Depth & 11 & 117718.6 & 117740.6 & 0.00 & 0.57 & 1.00 \\
GridArea + LeveeDist + CreekDist + ElevSD + Island + Depth & 12 & 117717.2 & 117741.2 & 0.60 & 0.42 & 1.35 \\
GridArea (null model) & 7 & 117834.6 & 117848.6 & 108.00 & $2.00 \mathrm{E}-24$ & $2.83 \mathrm{E}+23$ \\
$\quad$ Roosting & & & & & 0.31 \\
GridArea + ElevSD + Island + Depth & 10 & 145984.6 & 146004.6 & 0.00 & 1.00 \\
GridArea + Island + Depth & 9 & 145988.0 & 146006.0 & 1.40 & 0.15 & 2.01 \\
GridArea + LeveeDist + ElevSD + Island + Depth & 11 & 145984.2 & 146006.2 & 1.60 & 0.14 & 2.23 \\
GridArea + CreekDist + ElevSD + Island + Depth & 11 & 145984.2 & 146006.2 & 1.60 & 0.14 & 2.23 \\
GridArea + LeveeDist + Island + Depth & 10 & 145987.4 & 146007.4 & 2.80 & 0.08 & 4.06 \\
GridArea + CreekDist + Island + Depth & 10 & 145987.4 & 146007.4 & 2.80 & 0.08 & 4.06 \\
GridArea + LeveeDist + CreekDist + ElevSD + Island + Depth & 12 & 145983.8 & 146007.8 & 3.20 & 0.06 & 4.96 \\
GridArea (null model) & 7 & 146146.8 & 146160.8 & 156.20 & $3.76 \mathrm{E}-35$ & $8.28 \mathrm{E}+33$ \\
\hline
\end{tabular}


Table 39. The ranking of candidate models at the grid scale for foraging and roosting ruddy ducks in the former salt production ponds of South San Francisco Bay, California.

[Models are ranked by differences in Akaike's information criterion and only models of $\triangle \mathrm{AIC}<4$ and the null model are presented. Birds were surveyed across the former salt ponds during October through April 2002-2015. Variables considered included the mean grid depth (Depth), the presence of islands (Island), variation in topography (DepthSD), grid distance to the neared pond levee (LeveeDist), and grid distance to the nearest creak or slough (CreekDist). Grid area was included in every model. k: Number of estimated parameters in the model. AICc: Second-order Akaike's information criterion. $\triangle$ AICc: The difference between the AICc of the current model and the AICc of the top model. $\mathbf{w}_{\mathrm{i}}$ : Akaike weight-likelihood of the model relative to candidate models. Evidence ratio: The weight of evidence that the current model is inferior to the top model]

\begin{tabular}{lrrrrrr}
\hline \multicolumn{1}{c}{ Model name } & k & -2LogL & AlCc & AAICc & wi & $\begin{array}{c}\text { Evidence } \\
\text { ratio }\end{array}$ \\
\hline Foraging & & & & & & \\
GridArea + LeveeDist + Island + Depth & 10 & 96255.6 & 96275.6 & 0.00 & 0.50 & 1.00 \\
GridArea + LeveeDist + ElevSD + Island + Depth & 11 & 96255.2 & 96277.2 & 1.60 & 0.23 & 2.23 \\
GridArea + LeveeDist + CreekDist + Island + Depth & 11 & 96255.6 & 96277.6 & 2.00 & 0.19 & 2.72 \\
GridArea + LeveeDist + CreekDist + ElevSD + Island + Depth & 12 & 96255.2 & 96279.2 & 3.60 & 0.08 & 6.05 \\
GridArea (null model) & 7 & 96607.4 & 96621.4 & 345.80 & $4.10 \mathrm{E}-76$ & $1.23 \mathrm{E}+75$ \\
$\quad$ Roosting & & & & & & \\
GridArea + Island + Depth & 9 & 265590.0 & 265608.0 & 0.00 & 0.39 & 1.00 \\
GridArea + LeveeDist + Island + Depth & 10 & 265590.0 & 265610.0 & 2.00 & 0.14 & 2.72 \\
GridArea + CreekDist + Island + Depth & 10 & 265590.0 & 265610.0 & 2.00 & 0.14 & 2.72 \\
GridArea + ElevSD + Island + Depth & 10 & 265590.0 & 265610.0 & 2.00 & 0.14 & 2.72 \\
GridArea (null model) & 7 & 266616.0 & 266630.0 & 1022.00 & $4.65 \mathrm{E}-223$ & $8.40 \mathrm{E}+221$ \\
\hline
\end{tabular}

Table 40. The ranking of candidate models at the grid scale for foraging and roosting American avocets in the former salt production ponds of South San Francisco Bay, California.

[Models are ranked by differences in Akaike's information criterion and only models of $\triangle \mathrm{AIC}<4$ and the null model are presented. Birds were surveyed across the former salt ponds during October through April 2002-2015. Variables considered included the mean grid depth (Depth), the presence of islands (Island), variation in topography (DepthSD), grid distance to the neared pond levee (LeveeDist), and grid distance to the nearest creak or slough (CreekDist). Grid area was included in every model. k: Number of estimated parameters in the model. AICc: Second-order Akaike's information criterion. $\triangle$ AICc: The difference between the AICc of the current model and the AICc of the top model. wi: Akaike weight-likelihood of the model relative to candidate models. Evidence ratio: The weight of evidence that the current model is inferior to the top model]

\begin{tabular}{|c|c|c|c|c|c|c|}
\hline Model name & k & $-2 \log L$ & AICc & $\triangle \mathrm{AICc}$ & $\mathbf{w}_{\mathbf{i}}$ & $\begin{array}{c}\text { Evidence } \\
\text { ratio }\end{array}$ \\
\hline \multicolumn{7}{|l|}{ Foraging } \\
\hline GridArea + LeveeDist + CreekDist + ElevSD + Island + Depth & 12 & 49780.0 & 49804.0 & 0.00 & 0.72 & 1.00 \\
\hline GridArea + LeveeDist + CreekDist + Island + Depth & 11 & 49784.0 & 49806.0 & 2.00 & 0.27 & 2.72 \\
\hline GridArea (null model) & 7 & 50107.8 & 50121.8 & 317.80 & $7.06 \mathrm{E}-70$ & $1.02 \mathrm{E}+69$ \\
\hline \multicolumn{7}{|l|}{ Roosting } \\
\hline GridArea + LeveeDist + CreekDist + ElevSD + Island + Depth & 12 & 54598.6 & 54622.6 & 0 & 0.99 & 1 \\
\hline GridArea (null model) & 7 & 54954.8 & 54968.8 & 346.20 & $6.63 \mathrm{E}-76$ & $1.50 \mathrm{E}+75$ \\
\hline
\end{tabular}


Table 41. Variable importance values for significant model-averaged coefficients describing foraging and roosting waterbird guild abundance at the grid scale in restoration ponds, South San Francisco Bay, California.

[Numbers not highlighted indicate a significant positive relation, and numbers highlighted in gray indicate a significant negative relation. Higher values indicate increased importance of the variable for a given guild. "--" indicates no significant relation was observed.]

\begin{tabular}{|c|c|c|c|c|c|c|c|c|}
\hline Characteristic & $\begin{array}{l}\text { Dabbling } \\
\text { ducks }\end{array}$ & $\begin{array}{l}\text { Diving } \\
\text { ducks }\end{array}$ & $\begin{array}{l}\text { Medium } \\
\text { shorebirds }\end{array}$ & $\begin{array}{c}\text { Small } \\
\text { shorebirds }\end{array}$ & Gulls & Piscivores & Terns & Waders \\
\hline \multicolumn{9}{|c|}{ Foraging } \\
\hline Depth & 1 & 1 & 1 & 1 & 1 & 1 & 0.98 & 1 \\
\hline Islands & -- & -- & 1 & -- & -- & -- & -- & 1 \\
\hline Distance to creek & -- & -- & -- & 0.93 & -- & -- & -- & -- \\
\hline Distance to levees & -- & -- & 1 & 1 & 1 & 1 & 0.98 & 1 \\
\hline Topography & 0.92 & 0.95 & 1 & 1 & -- & -- & -- & 1 \\
\hline \multicolumn{9}{|c|}{ Roosting } \\
\hline Depth & 1 & 1 & 1 & 1 & 1 & 1 & 1 & 1 \\
\hline Islands & 0.95 & -- & 1 & 1 & 0.98 & 1 & 1 & 1 \\
\hline Distance to creek & -- & -- & 1 & -- & -- & 0.95 & -- & -- \\
\hline Distance to levees & -- & -- & 1 & 1 & 1 & 0.93 & 1 & 1 \\
\hline Topography & -- & -- & 0.95 & 1 & 1 & -- & -- & 0.97 \\
\hline
\end{tabular}

Table 42. Optimal habitat characteristic values for waterbird guilds at the grid scale, as determined from General Additive Models (GAMs), in restoration ponds, South San Francisco Bay, California.

[Variables with a linear trend (islands and breached) are not included here. “--” indicates no significant relation was observed. Abbreviations and symbol: km, kilometer; m, meter; >, greater than]

\begin{tabular}{lllllllll}
\hline & $\begin{array}{c}\text { Dabbling } \\
\text { ducks }\end{array}$ & $\begin{array}{c}\text { Diving } \\
\text { ducks }\end{array}$ & $\begin{array}{c}\text { Medium } \\
\text { shorebirds }\end{array}$ & $\begin{array}{c}\text { Small } \\
\text { shorebirds }\end{array}$ & Gulls & Piscivores & Terns & Waders \\
\hline & & \multicolumn{7}{c}{ Foraging } \\
\hline Depth (m) & 0.51 & 0.82 & 0 & 0 & 2.51 & $>0$ & 0.57 & 0.33 \\
Distance to creek (km) & -- & -- & -- & 0.04 & -- & -- & -- & -- \\
Distance to levees (km) & -- & -- & 0.05 & 0.05 & 0.05 & $>0.05$ & 0.06 & 0.08 \\
Topography (m) & 1.07 & 0.12 & 1.10 & 0.25 & -- & -- & -- & 1.25 \\
\hline & & \multicolumn{7}{c}{ Roosting } \\
\hline Depth (m) & 0.65 & 0.88 & 0 & 0 & 0 & 1.0 & 0.48 & 0.52 \\
Distance to creek (km) & -- & -- & 0.04 & -- & -- & 0.95 & -- & \\
Distance to levees (km) & -- & -- & 0.05 & 0.05 & $<0.45$ & 0.05 & 0.35 & 0.08 \\
Topography (m) & -- & -- & 1.10 & 0.25 & 0.84 & -- & -- & 0 \\
\hline
\end{tabular}


Table 43. Variable importance values for significant model-averaged coefficients describing foraging and roosting waterbird abundance at the grid scale in restoration ponds, South San Francisco Bay, California.

[Numbers not highlighted indicate a significant positive relation, and numbers highlighted in gray indicate a significant negative relation. Higher values indicate increased importance of the variable for a given species. "--" indicates no significant relation was observed. Abbreviations: American avocet, AMAV; eared grebe, EAGR; northern shoveler, NSHO; ruddy duck, RUDU]

\begin{tabular}{llccc}
\hline \multicolumn{1}{c}{ Characteristic } & AMAV & EAGR & NSHO & RUDU \\
\hline \multicolumn{5}{c}{ Foraging } \\
\hline Depth & 1 & 1 & 1 & 1 \\
Islands & 1 & -- & 1 & 1 \\
Distance to creek & 0.99 & -- & -- & -- \\
Distance to levees & 1 & 1 & 1 & 0.99 \\
Topography & -- & -- & 0.99 & -- \\
\hline \multicolumn{5}{c}{ Roosting } \\
\hline Depth & 1 & 1 & 1 & 1 \\
Islands & 1 & 0.99 & 1 & 1 \\
Distance to creek & 1 & -- & -- & -- \\
Distance to levees & 1 & 1 & -- & -- \\
Topography & 1 & -- & -- & -- \\
\hline
\end{tabular}

Table 44. Optimal habitat characteristic values for waterbird species at the grid scale, as determined from General Additive Models (GAMs), in restoration ponds, South San Francisco Bay, California.

[Variables with a linear trend (islands and breached), are not included here. "---" indicates no significant relation was observed. Abbreviations and symbol: American avocet, AMAV; eared grebe, EAGR; northern shoveler, NSHO; ruddy duck, RUDU; km, kilometer; m, meter; >, greater than]

\begin{tabular}{|c|c|c|c|c|}
\hline & AMAV & EAGR & NSHO & RUDU \\
\hline \multicolumn{5}{|c|}{ Foraging } \\
\hline Depth (m) & 0.03 & 1.5 & 0.42 & 0.78 \\
\hline Distance to creek $(\mathrm{km})$ & 0.04 & -- & -- & -- \\
\hline Distance to levees $(\mathrm{km})$ & 0.06 & 0.05 & $\begin{array}{l}0.06 \\
0 \text { and }\end{array}$ & 0.05 \\
\hline Topography (m) & -- & -- & 1.03 & -- \\
\hline \multicolumn{5}{|c|}{ Roosting } \\
\hline Depth (m) & 0.01 & 1.58 & 0.19 & 0.86 \\
\hline Distance to creek $(\mathrm{km})$ & 0.04 & -- & -- & -- \\
\hline Distance to levees $(\mathrm{km})$ & 0.07 & 0.04 & -- & -- \\
\hline Topography (m) & $>0.5$ & -- & -- & -- \\
\hline
\end{tabular}


Publishing support provided by the U.S. Geological Survey

Science Publishing Network, Tacoma Publishing Service Center

For more information concerning the research in this report, contact the Director, Western Ecological Research Center

U.S. Geological Survey

3020 State University Drive East

Sacramento, California 95819

https://www.werc.usgs.gov/ 
